Supporting Information for:

\title{
Nickel-Catalyzed Desymmetrizing Cyclization of 1,6-Dienes to Construct Quaternary Stereocenters
}

\author{
Tian-Yuan Zhao, Ke Li, Liang-Liang Yang, Shou-Fei Zhu, and Qi-Lin Zhou* \\ State Key Laboratory and Institute of Elemento-Organic Chemistry, College of Chemistry, \\ Nankai University, Tianjin 300071, China \\ E-mail:qlzhou@nankai.edu.cn
}

\section{Contents:}

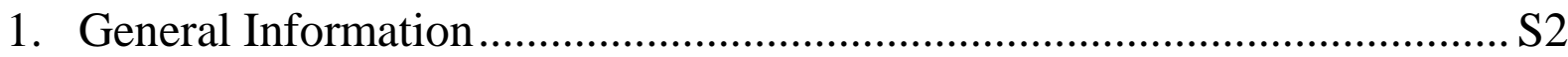

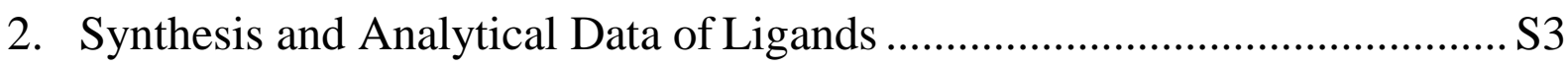

3. Synthesis and Analytical Data of 1,6-Diene Substrates ................................ S6

4. Typical Procedure for Desymmetrizing Cyclization of 1,6-Dienes ........... S19

5. Deuterium Experiments ……………………............................................ 31

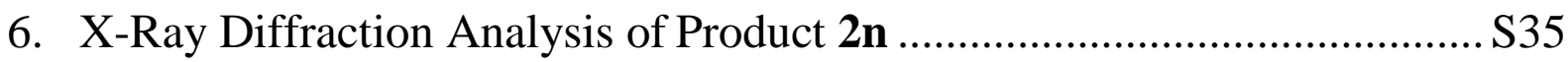

7. NMR Spectra of New Compounds ............................................................. S37

8. HPLC Charts of Products................................................................................... S93 


\section{General Information}

Methods: All reactions and manipulations which are sensitive to moisture or air were performed in an argon-filled glovebox (VAC DRI-LAB HE 493) or using standard Schlenk techniques. Melting points were measured on a RY-I apparatus and uncorrected. ${ }^{1} \mathrm{H}$ NMR and ${ }^{13} \mathrm{C}$ NMR spectra were recorded with a Brucker AV 400 spectrometer at $400 \mathrm{MHz}\left({ }^{1} \mathrm{H} \mathrm{NMR}\right)$ and $101 \mathrm{MHz}\left({ }^{13} \mathrm{C} \mathrm{NMR}\right)$ in $\mathrm{CDCl}_{3}$. Chemical shifts were reported in ppm down field from internal $\mathrm{Me}_{4} \mathrm{Si}$. Optical rotations were determined by a PerkinElmer $341 \mathrm{MC}$ polarimeter. HRMS were recorded on an Ion Spec FT-ICR mass spectrometer with ESI resource and Ion trap mass analyzer. HPLC analysis was performed on a Hewlett Packard Model HP 1100 Series chromatography. Crystal measurement was recorded with Rigaku XtaLAB P200 instrument.

Materials: Dichloromethane were freshly dried and distilled from $\mathrm{CaH}_{2}$. All chemicals reagents were purchased from Sterm, Acros and Sigma-Aldrich and used without further purification. [Ni(allyl)Br) $]_{2}$ was synthesized according to literature method. ${ }^{1}(R)-6,6$ '$\operatorname{di}\left(\left[1,1^{\prime}: 3\right.\right.$ ', $1^{\prime \prime}$-terphenyl]-5'-yl)-2,2',3,3'-tetrahydro-1,1'-spirobi[indene]-7,7'-diol was synthesized according to literature method. ${ }^{2}$ 


\section{Synthesis and Analytical Data of Ligands}
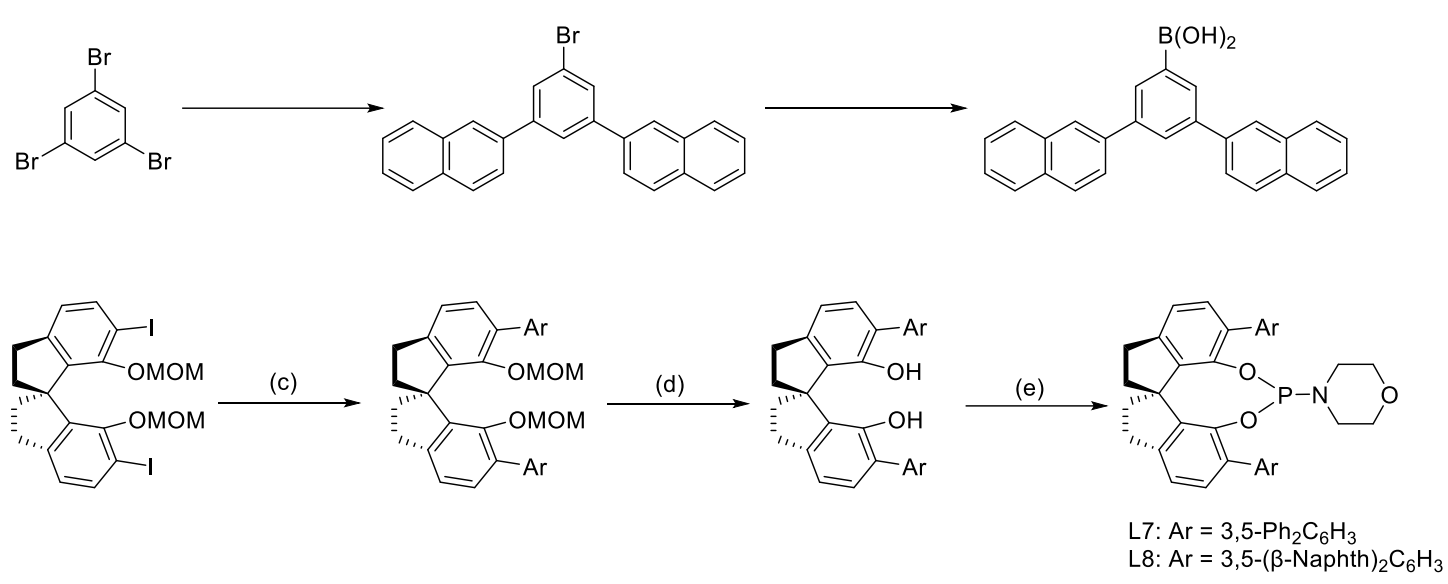

Reagents and condititions: (a) $\mathrm{Pd}\left(\mathrm{PPh}_{3}\right)_{4}, \mathrm{ArB}(\mathrm{OH})_{2}, \mathrm{~K}_{2} \mathrm{CO}_{3}, \mathrm{THF} / \mathrm{MeOH} / \mathrm{H}_{2} \mathrm{O}$, reflux, $30 \mathrm{~h}$; (b) i) $n$-BuLi, THF, $-78^{\circ} \mathrm{C}, 1 \mathrm{~h}$; ii) $\mathrm{B}\left(\mathrm{O}^{\prime} \mathrm{Pr}\right)_{3}, \mathrm{THF},-78{ }^{\circ} \mathrm{C}$, overnight; iii) $\mathrm{HCl}(3 \mathrm{M}), 1 \mathrm{~h}$; (c) $\mathrm{Pd}\left(\mathrm{PPh}_{3}\right)_{4}, \mathrm{ArB}(\mathrm{OH})_{2}, \mathrm{~K}_{2} \mathrm{CO}_{3}, \mathrm{THF} / \mathrm{MeOH} / \mathrm{H}_{2} \mathrm{O}$, reflux, $30 \mathrm{~h}$; (d) $\mathrm{HCl}$ (conc.), $\mathrm{CHCl}_{3} / \mathrm{MeOH}$, reflux, $4 \mathrm{~h}$; (e) i) $\mathrm{PCl}_{3}, \mathrm{Et}_{3} \mathrm{~N}, \mathrm{THF},-78{ }^{\circ} \mathrm{C}$ to r.t.; ii) morpholine, $-78{ }^{\circ} \mathrm{C}, 15 \mathrm{~min}$.

To a solution of tribromobenzene ( $3.1 \mathrm{~g}, 10 \mathrm{mmol}), \beta$-naphthylboronic acid (3.8 g, $22 \mathrm{mmol})$ and $\mathrm{Pd}\left(\mathrm{PPh}_{3}\right)_{4}(1.15 \mathrm{~g}, 1 \mathrm{mmol})$ in THF $(80 \mathrm{~mL})$ and $\mathrm{MeOH}(9 \mathrm{~mL})$, aqueous $\mathrm{K}_{2} \mathrm{CO}_{3}(3 \mathrm{M}, 33$ $\mathrm{mL}$ ) was added under the argon. The mixture was degassed and heated at reflux with heating mantle for $30 \mathrm{~h}$. After cooling to ambient temperature, the reaction mixture was diluted by $\mathrm{CH}_{2} \mathrm{Cl}_{2}$, the organic layer was washed with brine, dried over $\mathrm{MgSO}_{4}$, concentrated and purified by column chromatography (dichloromethane/petroleum ether, 1:20, v/v) to give 2,2'-(5bromo-1,3-phenylene)-dinaphthalene (2.8 g, 74\% yield) as a white solid.

A two-neck $500 \mathrm{~mL}$ flask fitted with a magnetic stirring bar was charged with 2,2'-(5-bromo1,3-phenylene)-dinaphthalene (2.8 g, $7 \mathrm{mmol})$ under argon atmosphere. Dry THF ( $80 \mathrm{~mL})$ was added, and the solution was cooled to $-78{ }^{\circ} \mathrm{C}$. To this solution was added $n$-BuLi $(3.5 \mathrm{~mL}, 2.5$ $\mathrm{M}, 8.4 \mathrm{mmol}$ ) using a slow addition pump over $30 \mathrm{~min}$. The solution was stirred at $-78^{\circ} \mathrm{C}$ for 1 $\mathrm{h}$ whereupon triisopropyl borate $(4.0 \mathrm{~g}, 21 \mathrm{mmol})$ dissolved in $20 \mathrm{~mL}$ of dry THF was added dropwise to the reaction system. The solution was stirred at $-78^{\circ} \mathrm{C}$ for $1 \mathrm{~h}$, then allowed to warm to room temperature overnight. After the reaction was quenched with dilute $\mathrm{HCl}(3 \mathrm{M}, 15$ $\mathrm{mL}$ ), the reaction mixture was stirred for $2 \mathrm{~h}$ at room temperature, and extracted with ethyl acetate $(3 \times 50 \mathrm{~mL})$. The organic layer was washed twice with brine and concentrated by rotary evaporation. To the residue (viscous liquid) was added methanol $(30 \mathrm{ml})$ and concentrated by rotary evaporation. The white (3,5-di(naphthalen-2-yl)phenyl)boronic acid was obtained and used without further purification.

To a solution of (R)-6,6'-diiodo-7,7'-bis(methoxymethoxy)-2,2',3,3'-tetrahydro-1,1'spirobi[indene] (1 mmol), (3,5-di(naphthalen-2-yl)phenyl)boronic acid (4 mmol), and $\mathrm{Pd}\left(\mathrm{PPh}_{3}\right)_{4}(173 \mathrm{mg}, 0.15 \mathrm{mmol})$ in THF $(60 \mathrm{~mL})$ and $\mathrm{MeOH}(5 \mathrm{~mL})$, aqueous $\mathrm{K}_{2} \mathrm{CO}_{3}(1 \mathrm{M}, 10$ $\mathrm{mL}$ ) were added under argon. The mixture was degassed and heated at reflux with heating 
mantle for $36 \mathrm{~h}$. After cooling to ambient temperature, the reaction mixture was diluted by ethyl acetate, washed with brine, dried over $\mathrm{MgSO}_{4}$ and purified by column chromatography (dichloromethane/petroleum ether, 1:5, v/v) to give $(R)-6,6$-bis(3,5-di(naphthalen-2yl)phenyl)-7,7'-bis(methoxymethoxy)-2,2',3,3'-tetrahydro-1,1'-spirobi[indene] (0.99 g, 99\% yield) as a white solid.

To a solution of (R)-7,7'-bis(methoxymethoxy)-6,6'-diaryl-2,2',3,3'-tetrahydro-1,1'spirobi[indene] (1 mmol) in $35 \mathrm{~mL} \mathrm{CHCl}_{3}$ and $15 \mathrm{~mL} \mathrm{MeOH}, \mathrm{HCl}$ (conc.) (10 mL) was added. The mixture was heated at reflux with heating mantle for $4 \mathrm{~h}$. After coolinig to ambient temperature, the reaction mixture was poured into brine, extracted by ethyl acetate. The combined organic phase was washed with saturated $\mathrm{NaHCO}_{3}$ and brine, dried over $\mathrm{MgSO}_{4}$. The solvent was removed by rotary evaporation, and the residue was purified by chromatography (dichloromethane/petroleum ether, 1:3, v/v) to give $(R)-6,6$-bis(3,5-di(naphthalen-2yl)phenyl)-2,2',3,3'-tetrahydro-1,1'-spirobi[indene]-7,7'-diol (0.86 g, 95\% yield) as a white solid.

To a chilled solution of $(R)$-6,6'-diaryl-2,2',3,3'-tetrahydro-1,1'-spirobi[indene]-7,7'-diol (454 $\mathrm{mg}, 0.5 \mathrm{mmol})$, Et3 $\mathrm{N}(152 \mathrm{mg}, 1.5 \mathrm{mmol})$ in THF $(10 \mathrm{~mL})$, a solution of $\mathrm{PCl}_{3}(137 \mathrm{mg}, 1 \mathrm{mmol})$ in THF ( $5 \mathrm{~mL}$ ) was added at $0{ }^{\circ} \mathrm{C}$ under argon. The mixture was stirred for $4 \mathrm{~h}$, and warmed to room temperature, and filtered under nitrogen. The filtrate was cooled to $-78{ }^{\circ} \mathrm{C}$ and treated with lithium morpholinate which was prepared from morpholine (87 $\mathrm{mg}, 1 \mathrm{mmol})$ and $n$-butyllithium (2.5 M in hexane, $440 \mu \mathrm{L}, 1.1 \mathrm{mmol}$ ) in $10 \mathrm{~mL}$ THF at $-78{ }^{\circ} \mathrm{C}$. The solution was stirred at $-78^{\circ} \mathrm{C}$ for $15 \mathrm{~min}$. The solvent was removed in vacuum and the residue was purified by column chromatography on silica gel (dichloromethane/petroleum ether, 1:5, v/v) to afford $(R)$-L8 (266 $\mathrm{mg}, 52 \%$ yield).

(R)-4-(1,10-bis(3,5-di(naphthalen-2-yl)phenyl)-4,5,6,7-tetrahydrodiindeno[7,1-de:1',7'fg] $[1,3,2]$ dioxaphosphocin-12-yl)morpholine ((R)-L8)

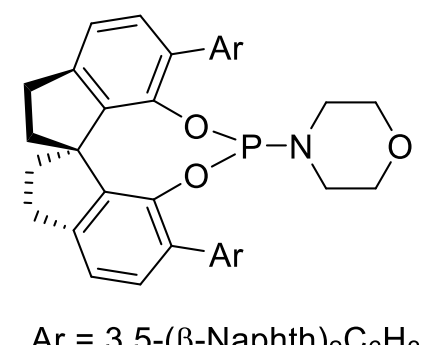

White solid, mp: $>300{ }^{\circ} \mathrm{C}$ (decompose). TLC $R_{f}=0.41(\mathrm{PE} / \mathrm{EA}=4: 1, \mathrm{v} / \mathrm{v}) .[\alpha]_{\mathrm{D}}^{25}+440(c 0.5$, $\left.\mathrm{CHCl}_{3}\right) .{ }^{1} \mathrm{H} \mathrm{NMR}\left(400 \mathrm{MHz}, \mathrm{CDCl}_{3}\right) \delta 8.10(\mathrm{~s}, 4 \mathrm{H}), 8.02(\mathrm{~s}, 2 \mathrm{H}), 7.96(\mathrm{~d}, J=6.2 \mathrm{~Hz}, 3 \mathrm{H}), 7.92$ 
- $7.79(\mathrm{~m}, 16 \mathrm{H}), 7.58-7.40(\mathrm{~m}, 10 \mathrm{H}), 7.22(\mathrm{~s}, 1 \mathrm{H}), 7.14(\mathrm{~d}, J=7.6 \mathrm{~Hz}, 1 \mathrm{H}), 3.16$ (tdd, $J=$ 16.8, 11.1, $6.5 \mathrm{~Hz}, 2 \mathrm{H}), 2.97(\mathrm{dd}, J=15.9,7.7 \mathrm{~Hz}, 2 \mathrm{H}), 2.92-2.75(\mathrm{~m}, 4 \mathrm{H}), 2.39$ (ddd, $J=$ 19.9, 12.0, 6.2 Hz, 2H), $2.31-2.11(\mathrm{~m}, 6 \mathrm{H}), 1.31-1.21(\mathrm{~m}, 4 \mathrm{H}), 0.87(\mathrm{qd}, J=7.9,7.0,3.0 \mathrm{~Hz}$, $4 \mathrm{H}) .{ }^{13} \mathrm{C}$ NMR $\left(101 \mathrm{MHz}, \mathrm{CDCl}_{3}\right) \delta 145.4(\mathrm{~d}, J=56.3 \mathrm{~Hz}), 144.0(\mathrm{~d}, J=7.2 \mathrm{~Hz}), 143.6(\mathrm{~d}, J=$ $3.9 \mathrm{~Hz}), 142.3,141.6(\mathrm{~d}, J=9.7 \mathrm{~Hz}), 139.7$ (d, $J=45.2 \mathrm{~Hz}), 138.6$ (d, $J=19.8 \mathrm{~Hz}), 134.2$ (d, $J=4.0 \mathrm{~Hz}), 133.4,133.3(\mathrm{~d}, J=98.3 \mathrm{~Hz}), 129.8,128.7,128.4,128.0$ (d, $J=48.0 \mathrm{~Hz}), 127.8$, $126.4(\mathrm{~d}, J=4.5 \mathrm{~Hz}), 126.1,125.8(\mathrm{t}, J=14.9 \mathrm{~Hz}), 125.2$ (d, $J=4.4 \mathrm{~Hz}), 121.5$ (d, $J=73.6$ $\mathrm{Hz}), 67.6(\mathrm{~d}, J=4.3 \mathrm{~Hz}), 59.9,43.3(\mathrm{~d}, J=18.6 \mathrm{~Hz}), 38.8(\mathrm{~d}, J=17.7 \mathrm{~Hz}), 30.7(\mathrm{~d}, J=46.9$ $\mathrm{Hz}) .{ }^{31} \mathrm{P}$ NMR $\left(162 \mathrm{MHz}, \mathrm{CDCl}_{3}\right) \delta$ 119.1. ESI-HRMS calcd for $\left[\mathrm{C}_{73} \mathrm{H}_{55} \mathrm{NO}_{3} \mathrm{P}^{+}, \mathrm{M}+\mathrm{H}\right]^{+}$: 1024.3914, Found 1024.3918.

(R)-4-(1,10-di([1,1':3',1''-terphenyl]-5'-yl)-4,5,6,7-tetrahydrodiindeno[7,1-de:1',7'fg] $[1,3,2]$ dioxaphosphocin-12-yl)morpholine ((R)-L7)

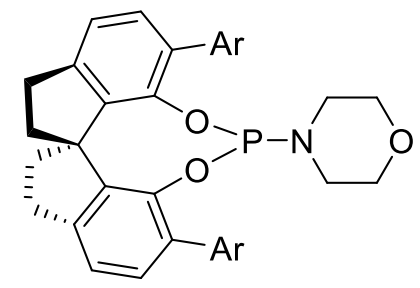

$\mathrm{Ar}=3,5-\mathrm{Ph}_{2} \mathrm{C}_{6} \mathrm{H}_{3}$

The ligand $(R)$-L7 was prepared from $(R)-6,6^{\prime}$-di([1, 1':3',1"-terphenyl]-5'-yl)-2,2',3,3'tetrahydro-1,1'-spirobi[indene]-7,7'-diol $(1.06 \mathrm{~g}, 1.5 \mathrm{mmol})$ by the same procedure as that for (R)-L8.

White solid, $880 \mathrm{mg}, 71 \%$ yield, $\mathrm{mp}:>300{ }^{\circ} \mathrm{C}$ (decompose). TLC $R_{f}=0.45$ (PE/EA $=4: 1$, v/v). $[\alpha]_{\mathrm{D}}{ }^{25}+425\left(c 0.5, \mathrm{CHCl}_{3}\right) .{ }^{1} \mathrm{H} \mathrm{NMR}\left(400 \mathrm{MHz}, \mathrm{CDCl}_{3}\right) \delta 7.83(\mathrm{~d}, J=25.1 \mathrm{~Hz}, 4 \mathrm{H}), 7.72$ (s, 1H), 7.63 (dd, $J=7.6,4.4 \mathrm{~Hz}, 9 \mathrm{H}), 7.51-7.37(\mathrm{~m}, 10 \mathrm{H}), 7.32$ (t, $J=7.4 \mathrm{~Hz}, 4 \mathrm{H}), 7.19$ (d, $J=7.7 \mathrm{~Hz}, 1 \mathrm{H}), 7.10(\mathrm{~d}, J=7.7 \mathrm{~Hz}, 1 \mathrm{H}), 3.14(\mathrm{tdd}, J=16.5,11.1,6.3 \mathrm{~Hz}, 2 \mathrm{H}), 2.92(\mathrm{td}, J=$ 15.6, 7.8 Hz, 2H), $2.84-2.68(\mathrm{~m}, 4 \mathrm{H}), 2.35(\mathrm{ddd}, J=19.7,11.9,6.3 \mathrm{~Hz}, 2 \mathrm{H}), 2.25-2.04(\mathrm{~m}$, $6 \mathrm{H}) .{ }^{13} \mathrm{C} \mathrm{NMR}\left(101 \mathrm{MHz}, \mathrm{CDCl}_{3}\right) \delta 145.6,145.0,143.9(\mathrm{~d}, J=7.3 \mathrm{~Hz}), 143.6(\mathrm{~d}, J=3.9 \mathrm{~Hz})$, $142.2,141.6(\mathrm{~d}, J=5.6 \mathrm{~Hz}), 141.3(\mathrm{~d}, J=20.6 \mathrm{~Hz}), 139.6,139.1,134.0$ (d, $J=4.4 \mathrm{~Hz}), 133.3$, $129.6(\mathrm{~d}, J=5.4 \mathrm{~Hz}), 128.9(\mathrm{~d}, J=2.2 \mathrm{~Hz}), 128.0,127.6-127.2(\mathrm{~m}), 124.7(\mathrm{~d}, J=12.9 \mathrm{~Hz})$, $121.8(\mathrm{~d}, J=2.2 \mathrm{~Hz}), 67.6,67.5,59.8,43.3,43.0,38.9,38.6,30.9,30.4 .{ }^{31} \mathrm{P}$ NMR $(162 \mathrm{MHz}$, $\left.\mathrm{CDCl}_{3}\right) \delta$ 118.8. ESI-HRMS calcd for $\left[\mathrm{C}_{57} \mathrm{H}_{47} \mathrm{NO}_{3} \mathrm{P}^{+}, \mathrm{M}+\mathrm{H}\right]^{+}:$824.3288, Found 824.3281. 


\section{Synthesis and Analytical Data of 1,6-Diene Substrates}

\subsection{General procedure for the preparation of isochromanone substrates}

\section{Method A}
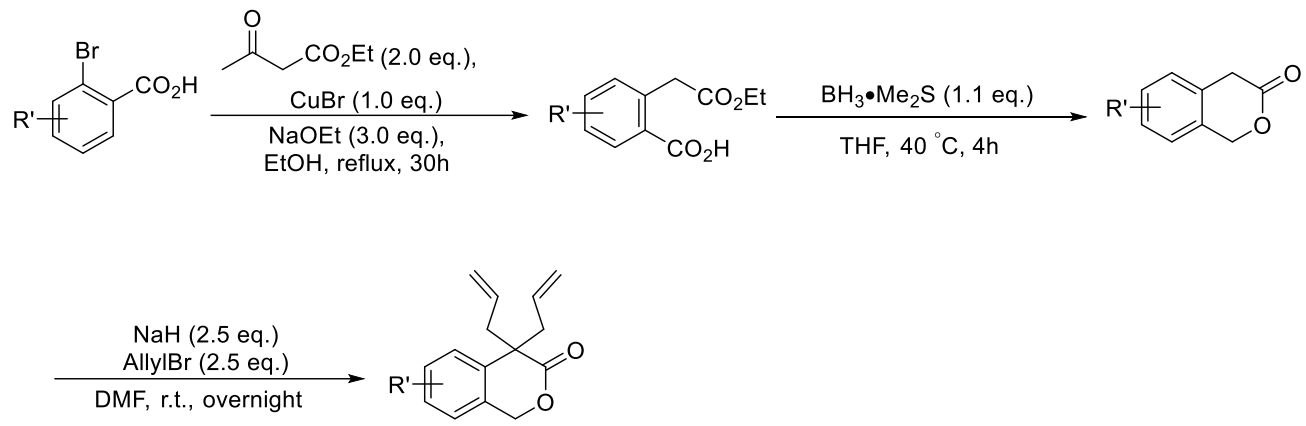

A flame-dried 2-neck flask was charged with 2-bromobenzoic acid (6.0 g, $30 \mathrm{mmol}), \mathrm{CuBr}$ $(5.1 \mathrm{~g}, 36 \mathrm{mmol})$ and $\mathrm{NaOEt}(6.1 \mathrm{~g}, 90 \mathrm{mmol})$, and sealed with a plastic cap under the argon. Ethanol $(50 \mathrm{ml})$ and ethyl acetoacetate $(7.8 \mathrm{~g}, 60 \mathrm{mmol})$ were added via syringe. The resulting suspension was heated at reflux with heating mantle for 30 hours. After cooling to room temperature, the reaction mixture was acidified to $\mathrm{pH} 1$ using concentrated hydrochloric acid. The layers were separated, and the aqueous phase was extracted with ethyl acetate $(3 \times 60 \mathrm{~mL})$. The combined organic phase was dried over anhydrous $\mathrm{Na}_{2} \mathrm{SO}_{4}$, and concentrated in vacuum. The residue was purified by column chromatography on silica gel (ethyl acetate/petroleum ether, 1:3-1:1, v/v) to afford 2-(2-ethoxy-2-oxoethyl)benzoic acid (4.2 g, 66\% yield).

To a stirred solution of the 2-(2-ethoxy-2-oxoethyl)benzoic acid (4.2 g, $20 \mathrm{mmol})$ in THF (30.0 mL) was added $10 \mathrm{M} \mathrm{BH}_{3} \cdot \mathrm{Me}_{2} \mathrm{~S}(2.2 \mathrm{~mL}, 22 \mathrm{mmol})$ under argon, and the mixture was heated to $40{ }^{\circ} \mathrm{C}$ with heating mantle for overnight. After cooling to room temperature, the reaction was quenched by addition of $\mathrm{HCl}$ (conc.) $(10 \mathrm{~mL})$. The mixture was extracted with ethyl acetate $(3 \times 60 \mathrm{~mL})$ and washed with brine $(20 \mathrm{~mL})$. The organic phase was dried over anhydrous $\mathrm{Na}_{2} \mathrm{SO}_{4}$ and concentrated. The residue was purified by flash chromatography (ethyl acetate /petroleum ether, 1:4-1:2, v/v) to give the isochroman-3-one (2.2 g, 75\% yield).

Sodium hydride $(1.5 \mathrm{~g}, 60 \mathrm{mmol})$ in dry DMF $(20 \mathrm{~mL})$ was cooled to $0{ }^{\circ} \mathrm{C}$ under the argon, the isochroman-3-one (2.2 g, $15 \mathrm{mmol})$ dissolved in $20 \mathrm{~mL}$ of dry DMF was added dropwise. The mixture was stirred at $0{ }^{\circ} \mathrm{C}$ for $1 \mathrm{~h}$, and then was allowed to warm to room temperature for $1 \mathrm{~h}$. The mixture was cooled to $0{ }^{\circ} \mathrm{C}$, allyl bromide $(7.2 \mathrm{~g}, 60 \mathrm{mmol})$ dissolved in $10 \mathrm{~mL}$ of dry DMF was added dropwise, and the mixture was allowed to warm to room temperature overnight. After cooled to $0{ }^{\circ} \mathrm{C}$, the reaction was quenched by adding saturated $\mathrm{NH}_{4} \mathrm{Cl}$ and extracted by ethyl acetate $(3 \times 60 \mathrm{~mL})$. The combined organic phase was washed with saturated $\mathrm{NH}_{4} \mathrm{Cl}$, dried over $\mathrm{Na}_{2} \mathrm{SO}_{4}$, and concentrated. The residue was purified by chromatography (ethyl 
acetate/petroleum ether, 1:4-1:2, v/v) to give pure 4,4-diallylisochroman-3-one (1a, $2.73 \mathrm{~g}, 80 \%$ yield).

\section{4,4-diallylisochroman-3-one (1a)}

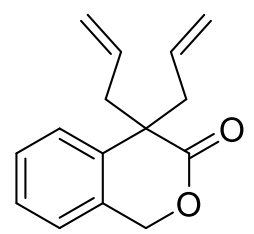

Colorless oil, TLC $R_{f}=0.54(\mathrm{PE} / \mathrm{EA}=5: 1, \mathrm{v} / \mathrm{v}) .{ }^{1} \mathrm{H}$ NMR $\left(400 \mathrm{MHz}, \mathrm{CDCl}_{3}\right) \delta 7.53-7.21$ (m, 3H), $7.11(\mathrm{~d}, J=7.6 \mathrm{~Hz}, 1 \mathrm{H}), 5.49$ (ddt, $J=17.3,10.2,7.1 \mathrm{~Hz}, 2 \mathrm{H}), 5.35$ (s, 2H), $5.16-$ $4.85(\mathrm{~m}, 4 \mathrm{H}), 2.88$ (ddt, $J=13.9,7.2,1.3 \mathrm{~Hz}, 2 \mathrm{H}), 2.64$ (ddt, $J=14.0,7.1,1.2 \mathrm{~Hz}, 2 \mathrm{H}) .{ }^{13} \mathrm{C}$ NMR $\left(101 \mathrm{MHz}, \mathrm{CDCl}_{3}\right) \delta 173.1,134.6,132.4,130.5,128.2,126.9,126.3,123.9,119.2,69.2$, 50.0, 43.1. ESI-HRMS calcd for $\left[\mathrm{C}_{15} \mathrm{H}_{16} \mathrm{NaO}_{2}{ }^{+}, \mathrm{M}+\mathrm{Na}\right]^{+}: 251.1043$, Found 251.1047.

\section{4,4-diallyl-7-methylisochroman-3-one (1b)}

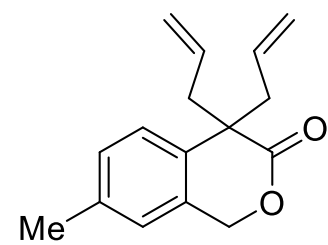

The 1b was prepared from 2-bromo-5-methylbenzoic acid (6.4 g, $30 \mathrm{mmol})$ by Method A (2.5 g, 34\% yield, 3 steps).

Colorless oil, TLC $R_{f}=0.53(\mathrm{PE} / \mathrm{EA}=5: 1, \mathrm{v} / \mathrm{v}) .{ }^{1} \mathrm{H}$ NMR $\left(400 \mathrm{MHz}, \mathrm{CDCl}_{3}\right) \delta 7.28-7.08$ (m, 2H), $6.92(\mathrm{~s}, 1 \mathrm{H}), 5.49$ (ddt, $J=17.2,10.2,7.2 \mathrm{~Hz}, 2 \mathrm{H}), 5.32(\mathrm{~s}, 2 \mathrm{H}), 5.11-4.81(\mathrm{~m}, 4 \mathrm{H})$, 2.87 (ddt, $J=13.9,7.2,1.2 \mathrm{~Hz}, 2 \mathrm{H}), 2.61(\mathrm{dd}, J=13.9,7.1 \mathrm{~Hz}, 2 \mathrm{H}), 2.35(\mathrm{~s}, 3 \mathrm{H}) .{ }^{13} \mathrm{C}$ NMR $\left(101 \mathrm{MHz}, \mathrm{CDCl}_{3}\right) \delta 173.6,136.9,132.8,131.8,130.7,129.3,126.5,124.6,119.4,69.5,50.1$, 43.4, 21.1. ESI-HRMS calcd for $\left[\mathrm{C}_{16} \mathrm{H}_{18} \mathrm{O}_{2} \mathrm{Na}^{+}, \mathrm{M}+\mathrm{Na}\right]^{+}:$265.1199, Found 265.1204.

\section{4,4-diallyl-7-chloroisochroman-3-one (1c)}<smiles>C=CCC1(CC=C)C(=O)OCc2cc(Cl)ccc21</smiles>

The 1c was prepared from 2-bromo-5-chlorobenzoic acid $(7.02 \mathrm{~g}, 30 \mathrm{mmol})$ by Method A (3.30 g, $42 \%$ yield, 3 steps).

Light green solid, mp: $57-59{ }^{\circ} \mathrm{C}$, TLC $R_{f}=0.50(\mathrm{PE} / \mathrm{EA}=5: 1, \mathrm{v} / \mathrm{v}) .{ }^{1} \mathrm{H}$ NMR $(400 \mathrm{MHz}$, 
$\left.\mathrm{CDCl}_{3}\right) \delta 7.35-7.23(\mathrm{~m}, 2 \mathrm{H}), 7.07(\mathrm{~d}, J=8.1 \mathrm{~Hz}, 1 \mathrm{H}), 5.48(\mathrm{ddt}, J=17.3,10.2,7.2 \mathrm{~Hz}, 2 \mathrm{H})$, $5.33(\mathrm{~s}, 2 \mathrm{H}), 5.07(\mathrm{t}, 4 \mathrm{H}), 2.90(\mathrm{dd}, J=14.0,7.2 \mathrm{~Hz}, 2 \mathrm{H}), 2.60(\mathrm{dd}, J=14.0,7.2 \mathrm{~Hz}, 2 \mathrm{H}) .{ }^{13} \mathrm{C}$ NMR $\left(101 \mathrm{MHz}, \mathrm{CDCl}_{3}\right) \delta 172.6,137.0,134.4,132.1,129.3,127.4,126.8,125.6,120.0,68.9$, 50.3, 43.4. ESI-HRMS calcd for $\left[\mathrm{C}_{15} \mathrm{H}_{15} \mathrm{ClO}_{2} \mathrm{Na}^{+}, \mathrm{M}+\mathrm{Na}\right]^{+}:$285.0653, Found 285.0658.

\section{4,4-diallyl-7-methoxyisochroman-3-one (1d)}

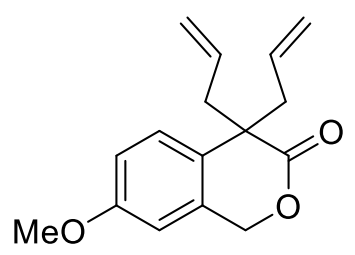

The 1d was prepared from 2-bromo-5-methoxybenzoic acid (6.90 g, $30 \mathrm{mmol})$ by Method A (3.50 g, $45 \%$ yield, 3 steps).

Colorless liquid, TLC $R_{f}=0.23(\mathrm{PE} / \mathrm{EA}=3: 1, \mathrm{v} / \mathrm{v}) .{ }^{1} \mathrm{H} \mathrm{NMR}\left(400 \mathrm{MHz}, \mathrm{CDCl}_{3}\right) \delta 7.21(\mathrm{~d}, J$ $=8.7 \mathrm{~Hz}, 1 \mathrm{H}), 6.91(\mathrm{dd}, J=8.7,2.7 \mathrm{~Hz}, 1 \mathrm{H}), 6.61(\mathrm{~d}, 1 \mathrm{H}), 5.50(\mathrm{ddt}, J=17.2,10.1,7.2 \mathrm{~Hz}$, 2H), $5.32(\mathrm{~s}, 2 \mathrm{H}), 5.11-4.89(\mathrm{~m}, 4 \mathrm{H}), 3.81(\mathrm{~s}, 3 \mathrm{H}), 2.86(\mathrm{dd}, J=13.9,7.2 \mathrm{~Hz}, 2 \mathrm{H}), 2.60$ (dd, $J=14.0,7.2 \mathrm{~Hz}, 2 \mathrm{H}) .{ }^{13} \mathrm{C} \mathrm{NMR}\left(101 \mathrm{MHz}, \mathrm{CDCl}_{3}\right) \delta 173.6,158.5,132.8,132.0,128.0,126.6$, 119.4, 114.6, 108.7, 69.4, 55.4, 49.8, 43.6. ESI-HRMS calcd for $\left[\mathrm{C}_{16} \mathrm{H}_{18} \mathrm{NaO}_{3}{ }^{+}, \mathrm{M}+\mathrm{Na}\right]^{+}$: 281.1148, Found 281.1152.

\section{4,4-diallyl-6-methylisochroman-3-one (1e)}<smiles>C=CCC1(CC=C)C(=O)OCc2ccc(C)cc21</smiles>

The 1e was prepared from 2-bromo-4-methylbenzoic acid $(6.42 \mathrm{~g}, 30 \mathrm{mmol})$ by Method A (3.0 g, 41\% yield, 3 steps).

Colorless liquid, TLC $R_{f}=0.53(\mathrm{PE} / \mathrm{EA}=5: 1, \mathrm{v} / \mathrm{v}) .{ }^{1} \mathrm{H} \mathrm{NMR}\left(400 \mathrm{MHz}, \mathrm{CDCl}_{3}\right) \delta 7.13-$ $7.05(\mathrm{~m}, 2 \mathrm{H}), 7.00$ (d, $J=7.7 \mathrm{~Hz}, 1 \mathrm{H}), 5.50$ (ddt, $J=17.2,10.0,7.2 \mathrm{~Hz}, 2 \mathrm{H}), 5.33(\mathrm{~s}, 2 \mathrm{H}), 5.11$ $-4.90(\mathrm{~m}, 4 \mathrm{H}), 2.91-2.83(\mathrm{~m}, 2 \mathrm{H}), 2.63(\mathrm{ddd}, J=13.9,6.9,1.2 \mathrm{~Hz}, 2 \mathrm{H}), 2.38(\mathrm{~s}, 3 \mathrm{H}) .{ }^{13} \mathrm{C}$ NMR $\left(101 \mathrm{MHz}, \mathrm{CDCl}_{3}\right) \delta 173.5,138.1,134.7,132.7,127.9,127.8,126.9,124.0,119.3,69.3$, 50.3, 43.3, 21.6. ESI-HRMS calcd for $\left[\mathrm{C}_{16} \mathrm{H}_{18} \mathrm{NaO}_{2}{ }^{+}, \mathrm{M}+\mathrm{Na}\right]^{+}:$265.1199, Found 265.1203.

\section{4,4-diallyl-6-chloroisochroman-3-one (1f)}




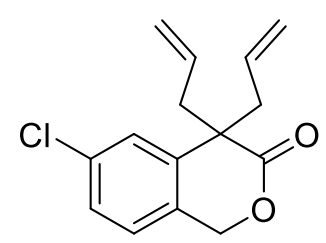

The 1f was prepared from 2-bromo-4-chlorobenzoic acid (7.0 g, $30 \mathrm{mmol})$ by Method A (3.3 g, $42 \%$ yield, 3 steps).

Light green solid, mp: $58-60{ }^{\circ} \mathrm{C}$, TLC $R_{f}=0.53(\mathrm{PE} / \mathrm{EA}=3: 1, \mathrm{v} / \mathrm{v}) .{ }^{1} \mathrm{H}$ NMR $(400 \mathrm{MHz}$, $\left.\mathrm{CDCl}_{3}\right) \delta 7.52-7.24(\mathrm{~m}, 2 \mathrm{H}), 7.07(\mathrm{~d}, J=8.1 \mathrm{~Hz}, 1 \mathrm{H}), 5.48(\mathrm{ddt}, J=17.3,10.2,7.2 \mathrm{~Hz}, 2 \mathrm{H})$, $5.33(\mathrm{~s}, 2 \mathrm{H}), 5.15-4.94(\mathrm{~m}, 4 \mathrm{H}), 2.90(\mathrm{dd}, J=14.0,7.2 \mathrm{~Hz}, 2 \mathrm{H}), 2.60(\mathrm{dd}, J=14.0,7.2 \mathrm{~Hz}$, $2 \mathrm{H}) .{ }^{13} \mathrm{C} \mathrm{NMR}\left(101 \mathrm{MHz}, \mathrm{CDCl}_{3}\right) \delta 172.6,137.0,134.4,132.1,129.3,127.4,126.7,125.6$, 120.0, 68.9, 50.3, 43.3. ESI-HRMS calcd for $\left[\mathrm{C}_{15} \mathrm{H}_{15} \mathrm{ClNaO}_{2}{ }^{+}, \mathrm{M}+\mathrm{Na}\right]^{+}: 285.0653$, Found 285.0656 .

\section{4,4-diallyl-6-methoxyisochroman-3-one (1g)}

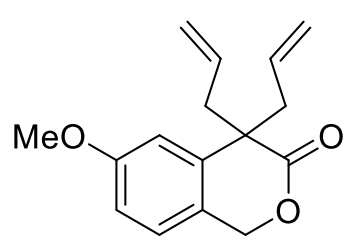

The $1 \mathrm{~g}$ was prepared from 2-bromo-4-methoxybenzoic acid $(7.0 \mathrm{~g}, 30 \mathrm{mmol})$ by Method A (2.7 g, 35\% yield, 3 steps).

Colorless liquid, TLC $R_{f}=0.48(\mathrm{PE} / \mathrm{EA}=3: 1, \mathrm{v} / \mathrm{v}) .{ }^{1} \mathrm{H}$ NMR $\left(400 \mathrm{MHz}, \mathrm{CDCl}_{3}\right) \delta 7.16-$ $7.01(\mathrm{~m}, 1 \mathrm{H}), 6.83(\mathrm{dq}, J=4.1,2.5 \mathrm{~Hz}, 2 \mathrm{H}), 5.52$ (ddt, $J=17.2,10.1,7.2 \mathrm{~Hz}, 1 \mathrm{H}), 5.31(\mathrm{~s}, 2 \mathrm{H})$, $5.19-4.88(\mathrm{~m}, 3 \mathrm{H}), 3.82(\mathrm{~s}, 3 \mathrm{H}), 2.87(\mathrm{ddt}, J=14.0,7.2,1.3 \mathrm{~Hz}, 2 \mathrm{H}), 2.74-2.52(\mathrm{~m}, 2 \mathrm{H}) .{ }^{13} \mathrm{C}$ NMR (101 MHz, $\left.\mathrm{CDCl}_{3}\right) \delta 173.1,159.6,136.3,132.6,125.3,123.0,119.3,112.5,112.2,69.0$, 55.4, 50.3, 43.0. ESI-HRMS calcd for $\left[\mathrm{C}_{16} \mathrm{H}_{18} \mathrm{NaO}_{3}{ }^{+}, \mathrm{M}+\mathrm{Na}\right]^{+}:$281.1148, Found 281.1151.

\section{4,4-diallyl-6-bromoisochroman-3-one (1h)}

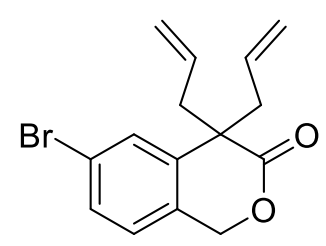

The $\mathbf{1 h}$ was prepared from 2,4-dibromobenzoic acid $(8.3 \mathrm{~g}, 30 \mathrm{mmol})$ by Method A (4.3 g, $45 \%$ yield, 3 steps).

White solid, mp: $68-70{ }^{\circ} \mathrm{C}$, TLC $R_{f}=0.26(\mathrm{PE} / \mathrm{EA}=5: 1, \mathrm{v} / \mathrm{v}) .{ }^{1} \mathrm{H}$ NMR $\left(400 \mathrm{MHz}, \mathrm{CDCl}_{3}\right)$ $\delta 7.61-7.32(\mathrm{~m}, 2 \mathrm{H}), 7.01(\mathrm{~d}, J=8.1 \mathrm{~Hz}, 1 \mathrm{H}), 5.48(\mathrm{ddt}, J=17.3,10.1,7.2 \mathrm{~Hz}, 2 \mathrm{H}), 5.31$ (s, 
$2 \mathrm{H}), 5.14-4.86(\mathrm{~m}, 4 \mathrm{H}), 2.90(\mathrm{dd}, J=14.0,7.2 \mathrm{~Hz}, 2 \mathrm{H}), 2.59(\mathrm{dd}, J=14.0,7.2 \mathrm{~Hz}, 2 \mathrm{H}) .{ }^{13} \mathrm{C}$ $\operatorname{NMR}\left(101 \mathrm{MHz}, \mathrm{CDCl}_{3}\right) \delta 172.5,137.4,132.1,130.3,129.8,129.7,125.8,122.4,120.0,68.9$, 50.3, 43.4. ESI-HRMS calcd for $\left[\mathrm{C}_{15} \mathrm{H}_{15} \mathrm{BrNaO}_{2}{ }^{+}, \mathrm{M}+\mathrm{Na}\right]^{+}:$329.0148, Found 329.0151.

\section{4,4-diallyl-6-fluoroisochroman-3-one (1i)}<smiles>C=CCC1(CC=C)C(=O)OCc2ccc(F)cc21</smiles>

The 1i was prepared from 2-bromo-4-fluorobenzoic acid (6.5 g, $30 \mathrm{mmol})$ by Method A (2.4 g, $32 \%$ yield, 3 steps)

Colorless liquid, TLC $R_{f}=0.40(\mathrm{PE} / \mathrm{EA}=5: 1, \mathrm{v} / \mathrm{v}) .{ }^{1} \mathrm{H}$ NMR $\left(400 \mathrm{MHz}, \mathrm{CDCl}_{3}\right) \delta 7.11(\mathrm{ddd}$, $J=7.7,5.6,1.1 \mathrm{~Hz}, 1 \mathrm{H}), 7.05-6.89(\mathrm{~m}, 2 \mathrm{H}), 5.49$ (ddt, $J=17.2,10.1,7.2 \mathrm{~Hz}, 2 \mathrm{H}), 5.34$ (s, $2 \mathrm{H}), 5.12-4.98(\mathrm{~m}, 4 \mathrm{H}), 2.90(\mathrm{dd}, J=14.1,7.2 \mathrm{~Hz}, 2 \mathrm{H}), 2.59(\mathrm{dd}, J=14.0,7.2 \mathrm{~Hz}, 2 \mathrm{H}) .{ }^{13} \mathrm{C}$ $\operatorname{NMR}\left(101 \mathrm{MHz}, \mathrm{CDCl}_{3}\right) \delta 172.6,164.0,137.6(\mathrm{~d}, J=7.4 \mathrm{~Hz}), 132.2,126.7(\mathrm{~d}, J=3.0 \mathrm{~Hz})$, $126.0(\mathrm{~d}, J=8.6 \mathrm{~Hz}), 119.9,114.5(\mathrm{~d}, J=22.2 \mathrm{~Hz}), 113.6(\mathrm{~d}, J=22.8 \mathrm{~Hz}), 68.9,50.4(\mathrm{~d}, J=$ 1.6 Hz), 43.2. ${ }^{19} \mathrm{~F} \mathrm{NMR}\left(376 \mathrm{MHz}, \mathrm{CDCl}_{3}\right) \delta$-112.8. ESI-HRMS calcd for $\left[\mathrm{C}_{15} \mathrm{H}_{15} \mathrm{FNaO}_{2}{ }^{+}, \mathrm{M}\right.$ $+\mathrm{Na}]^{+}:$269.0948, Found 269.0953.

\section{4,4-diallyl-6-(trifluoromethyl)isochroman-3-one (1j)}<smiles>C=CCC1(CC=C)C(=O)OCc2ccc(C(F)(F)F)cc21</smiles>

The 1j was prepared from 2-bromo-4-(trifluoromethyl)benzoic acid (8.0 g, $30 \mathrm{mmol})$ by Method A (2.8 g, 32\% yield, 3 steps).

White solid, mp: 59-61 ${ }^{\circ} \mathrm{C}$, TLC $R_{f}=0.40(\mathrm{PE} / \mathrm{EA}=5: 1, \mathrm{v} / \mathrm{v}) .{ }^{1} \mathrm{H}$ NMR $\left(400 \mathrm{MHz}, \mathrm{CDCl}_{3}\right)$ $\delta 7.56(\mathrm{t}, J=3.5 \mathrm{~Hz}, 2 \mathrm{H}), 7.27(\mathrm{~d}, J=7.9 \mathrm{~Hz}, 1 \mathrm{H}), 5.55-5.43(\mathrm{~m}, 2 \mathrm{H}), 5.42(\mathrm{~s}, 2 \mathrm{H}), 5.22-$ $4.91(\mathrm{~m}, 4 \mathrm{H}), 2.94(\mathrm{dd}, J=14.1,7.1 \mathrm{~Hz}, 2 \mathrm{H}), 2.65(\mathrm{dd}, J=14.0,7.4 \mathrm{~Hz}, 2 \mathrm{H}) .{ }^{13} \mathrm{C} \mathrm{NMR}(101$ $\left.\mathrm{MHz}, \mathrm{CDCl}_{3}\right) \delta 172.4,136.1,134.6,131.9,130.8(\mathrm{q}, J=32.5 \mathrm{~Hz}), 124.8,123.9$ (dq, $J=15.1$, $3.7 \mathrm{~Hz}), 123.9(\mathrm{~d}, J=272.3 \mathrm{~Hz}), 120.2,68.9,50.4,43.4 .{ }^{19} \mathrm{~F} \mathrm{NMR}\left(376 \mathrm{MHz}, \mathrm{CDCl}_{3}\right) \delta-67.4$. ESI-HRMS calcd for $\left[\mathrm{C}_{16} \mathrm{H}_{15} \mathrm{~F}_{3} \mathrm{NaO}_{2}{ }^{+}, \mathrm{M}+\mathrm{Na}\right]^{+}:$319.0916, Found 319.0920.

\section{8,8-diallyl-5,8-dihydro-7H-[1,3]dioxolo[4,5-g]isochromen-7-one (10)}




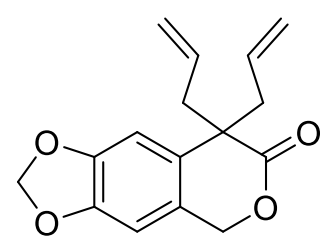

The 10 was prepared from 6-bromobenzo[d][1,3]dioxole-5-carboxylic acid (8.0 g, $30 \mathrm{mmol})$ by Method A (3.7 g, 50\% yield, 3 steps).

White solid, mp: $55-57{ }^{\circ} \mathrm{C}$, TLC $R_{f}=0.45(\mathrm{PE} / \mathrm{EA}=5: 1, \mathrm{v} / \mathrm{v}) .{ }^{1} \mathrm{H} \mathrm{NMR}\left(400 \mathrm{MHz}, \mathrm{CDCl}_{3}\right)$ $\delta 6.75(\mathrm{~s}, 1 \mathrm{H}), 6.54(\mathrm{~s}, 1 \mathrm{H}), 5.99$ (s, 2H), 5.51 (ddt, $J=17.1,10.1,7.2 \mathrm{~Hz}, 2 \mathrm{H}), 5.24(\mathrm{~s}, 2 \mathrm{H})$, $5.04(\mathrm{dd}, J=19.3,13.6 \mathrm{~Hz}, 4 \mathrm{H}), 2.86(\mathrm{dd}, J=14.0,7.2 \mathrm{~Hz}, 2 \mathrm{H}), 2.54(\mathrm{dd}, J=14.0,7.2 \mathrm{~Hz}$, 2H). ${ }^{13} \mathrm{C}$ NMR $\left(101 \mathrm{MHz}, \mathrm{CDCl}_{3}\right) \delta 173.2,148.2,146.9,132.6,128.3,124.3,119.5,106.5$, 104.1, 101.6, 69.3, 50.3, 43.6. ESI-HRMS calcd for $\left[\mathrm{C}_{16} \mathrm{H}_{16} \mathrm{NaO}_{4}{ }^{+}, \mathrm{M}+\mathrm{Na}\right]^{+}:$295.0941, Found 295.0944 .

\section{Method B ${ }^{4}$}
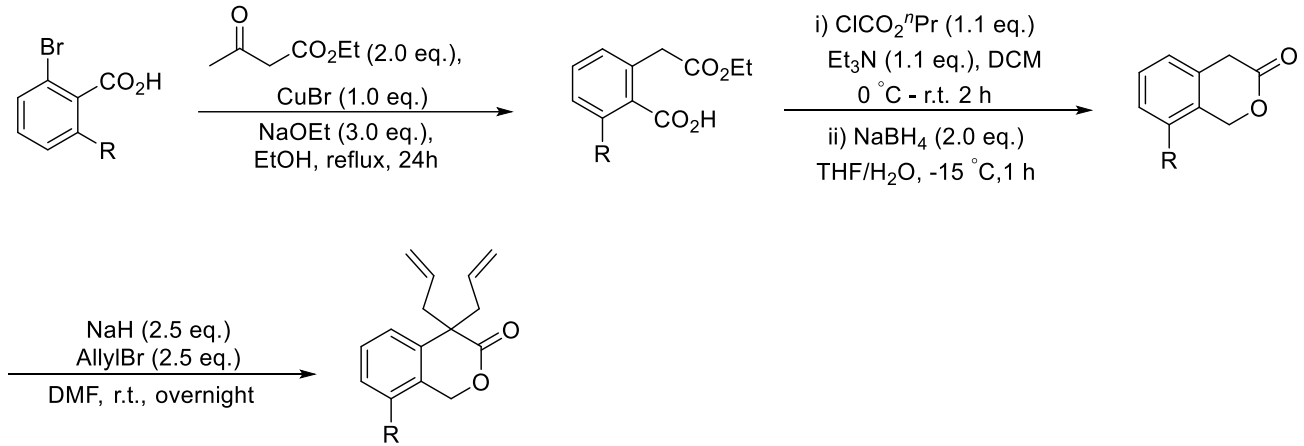

A flame-dried 2-neck flask was added 2-bromo-6-fluorobenzoic acid (6.5 g, $30 \mathrm{mmol}), \mathrm{CuBr}$ $(5.1 \mathrm{~g}, 36 \mathrm{mmol})$, and $\mathrm{NaOEt}(6.1 \mathrm{~g}, 90 \mathrm{mmol})$ under argon. Ethanol $(50 \mathrm{ml})$ and ethyl acetoacetate $(7.8 \mathrm{~g}, 60 \mathrm{mmol})$ were added via syringe. The resulting suspension was heated at reflux with heating mantle for 30 hours. After cooling to room temperature, the reaction mixture was acidified to $\mathrm{pH} 1$ using concentrated $\mathrm{HCl}$. The layers were separated, and aqueous layer was extracted with ethyl acetate $(3 \times 60 \mathrm{~mL})$. The combined organic phase was dried over $\mathrm{Na}_{2} \mathrm{SO}_{4}$ and concentrated. The residue was purified by column chromatography on silica gel (ethyl acetate/petroleum ether, 1:3-1:1, v/v) to afford 2-(2-ethoxy-2-oxoethyl)-6-fluorobenzoic acid (5.2 g, 77\% yield).

$n$-Propyl chloroformate $(2.68 \mathrm{~g}, 22 \mathrm{mmol})$ was added to a solution of 2-(2-ethoxy-2oxoethyl)-6-fluorobenzoic acid (5.0 g, $20 \mathrm{mmol})$ and $\mathrm{Et}_{3} \mathrm{~N}(6.4 \mathrm{~mL}, 46 \mathrm{mmol})$ in $\mathrm{CH}_{2} \mathrm{Cl}_{2}(84$ $\mathrm{mL}$ ) at $0{ }^{\circ} \mathrm{C}$ under argon. After stirring for $2 \mathrm{~h}$ at room temperature, the reaction was quenched by addition of $2 \mathrm{M} \mathrm{HCl}$ and the mixture was extracted with $\mathrm{CH}_{2} \mathrm{Cl}_{2}$. The organic layers were washed with brine, dried $\left(\mathrm{Na}_{2} \mathrm{SO}_{4}\right)$ and concentrated. The crude was dissolved in THF (100 
$\mathrm{mL})$, and a cold $\left(0{ }^{\circ} \mathrm{C}\right)$ solution of $\mathrm{NaBH}_{4}(3.2 \mathrm{~g}, 84 \mathrm{mmol})$ in water $(34 \mathrm{~mL})$ was added at $15{ }^{\circ} \mathrm{C}$. After stirring at $-15{ }^{\circ} \mathrm{C}$ for $1 \mathrm{~h}$, the reaction was quenched by addition of $1 \mathrm{M} \mathrm{HCl}$ and extracted with ethyl acetate $(3 \times 50 \mathrm{~mL})$. The organic layers were washed with brine, dried over $\mathrm{Na}_{2} \mathrm{SO}_{4}$ and concentrated. The residue was purified by chromatography (ethyl acetate /petroleum ether, $1: 5, \mathrm{v} / \mathrm{v})$ to give 8-fluoroisochroman-3-one $(2.5 \mathrm{~g}, 73 \%$ yield $)$.

Sodium hydride $(1.5 \mathrm{~g}, 60 \mathrm{mmol})$ in dry DMF $(20 \mathrm{~mL})$ was cooled to $0{ }^{\circ} \mathrm{C}$ under the argon, the 8-fluoroisochroman-3-one (2.5 g, $15 \mathrm{mmol})$ dissolved in $20 \mathrm{~mL}$ of dry DMF was added dropwise. The mixture was stirred at $0{ }^{\circ} \mathrm{C}$ for $1 \mathrm{~h}$, and then was allowed to warm to room temperature for $1 \mathrm{~h}$. The mixture was cooled to $0{ }^{\circ} \mathrm{C}$, allyl bromide $(7.2 \mathrm{~g}, 60 \mathrm{mmol})$ dissolved in $10 \mathrm{~mL}$ of dry DMF was added dropwise. The mixture allowed to warm to room temperature overnight. After cooled to $0{ }^{\circ} \mathrm{C}$, the reaction mixture was quenched by adding saturated $\mathrm{NH}_{4} \mathrm{Cl}$, extracted by ethyl acetate. The combined organic phase was washed with saturated $\mathrm{NH}_{4} \mathrm{Cl}$, dried over $\mathrm{Na}_{2} \mathrm{SO}_{4}$ and concentrated. The residue was purified by chromatography (ethyl acetate/petroleum ether, 1/4-1/2, v/v) to give pure 4,4-diallyl-8-fluoroisochroman-3-one (1n, $2.8 \mathrm{~g}, 76 \%$ yield).

\section{4,4-diallyl-8-fluoroisochroman-3-one (1n)}

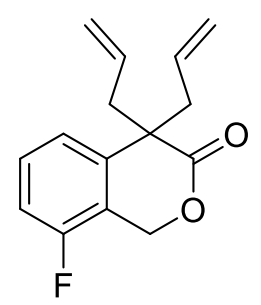

Colorless liquid, TLC $R_{f}=0.68(\mathrm{PE} / \mathrm{EA}=4: 1, \mathrm{v} / \mathrm{v}) .{ }^{1} \mathrm{H} \mathrm{NMR}\left(400 \mathrm{MHz}, \mathrm{CDCl}_{3}\right) \delta 7.36(\mathrm{td}$, $J=8.1,5.7 \mathrm{~Hz}, 1 \mathrm{H}), 7.11(\mathrm{~d}, J=7.9 \mathrm{~Hz}, 1 \mathrm{H}), 6.99(\mathrm{t}, J=8.8 \mathrm{~Hz}, 1 \mathrm{H}), 5.55-5.44(\mathrm{~m}, 2 \mathrm{H})$, $5.42(\mathrm{~s}, 2 \mathrm{H}), 5.09-4.95(\mathrm{~m}, 4 \mathrm{H}), 2.92(\mathrm{dd}, J=13.9,7.3 \mathrm{~Hz}, 2 \mathrm{H}), 2.62(\mathrm{dd}, J=13.9,7.2 \mathrm{~Hz}$, $2 \mathrm{H}) .{ }^{13} \mathrm{C} \mathrm{NMR}\left(101 \mathrm{MHz}, \mathrm{CDCl}_{3}\right) \delta 172.7,159.1,137.7(\mathrm{~d}, J=3.0 \mathrm{~Hz}), 132.3,129.8(\mathrm{~d}, J=$ $8.2 \mathrm{~Hz}), 122.1(\mathrm{~d}, J=3.6 \mathrm{~Hz}), 119.8,118.8(\mathrm{~d}, J=16.3 \mathrm{~Hz}), 113.5(\mathrm{~d}, J=20.0 \mathrm{~Hz}), 64.2(\mathrm{~d}, J$ $=5.8 \mathrm{~Hz}), 50.3(\mathrm{~d}, J=2.0 \mathrm{~Hz}), 44.0 .{ }^{19} \mathrm{~F} \mathrm{NMR}\left(376 \mathrm{MHz}, \mathrm{CDCl}_{3}\right) \delta-129.8$. ESI-HRMS calcd for $\left[\mathrm{C}_{15} \mathrm{H}_{15} \mathrm{FNaO}_{2}{ }^{+}, \mathrm{M}+\mathrm{Na}\right]^{+}:$269.0948, Found 269.0952.

\section{4,4-diallyl-8-methylisochroman-3-one (1m)}




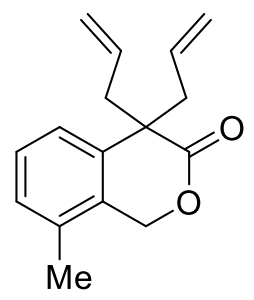

The 1m was prepared from 2-bromo-6-methylbenzoic acid (6.4 g, $30 \mathrm{mmol})$ by Method B (1.5 g, 21\% yield, 3 steps).

Colorless liquid, TLC $R_{f}=0.41(\mathrm{PE} / \mathrm{EA}=5: 1, \mathrm{v} / \mathrm{v}) .{ }^{1} \mathrm{H}$ NMR $\left(400 \mathrm{MHz}, \mathrm{CDCl}_{3}\right) \delta 7.27(\mathrm{t}, J$ $=7.7 \mathrm{~Hz}, 1 \mathrm{H}), 7.16(\mathrm{~d}, J=7.9 \mathrm{~Hz}, 1 \mathrm{H}), 7.09(\mathrm{~d}, J=7.4 \mathrm{~Hz}, 1 \mathrm{H}), 5.48$ (ddt, $J=17.3,10.2,7.2$ $\mathrm{Hz}, 2 \mathrm{H}), 5.34$ (s, 2H), $5.07-4.96(\mathrm{~m}, 4 \mathrm{H}), 2.89$ (dd, $J=13.8,7.4 \mathrm{~Hz}, 2 \mathrm{H}), 2.62$ (dd, $J=13.9$, $7.0 \mathrm{~Hz}, 2 \mathrm{H}), 2.23$ (s, 3H). ${ }^{13} \mathrm{C} \mathrm{NMR}\left(101 \mathrm{MHz}, \mathrm{CDCl}_{3}\right) \delta 173.2,134.6,132.7,132.7,128.9$, 128.7, 128.2, 124.2, 119.3, 67.8, 50.0, 44.0, 18.5. ESI-HRMS calcd for $\left[\mathrm{C}_{16} \mathrm{H}_{18} \mathrm{NaO}_{2}{ }^{+}, \mathrm{M}+\right.$ $\mathrm{Na}]^{+}:$265.1199, Found 265.1201.

\section{Method C}<smiles>C=CCC1(CC=C)C(=O)OCc2ccc(Br)cc21</smiles>

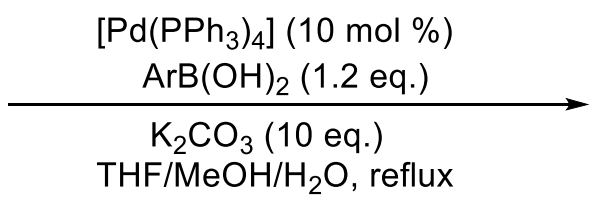<smiles>[R]c1ccc2c(c1)C(CC=C)(CC=C)C(=O)OC2</smiles>

$81 \%-94 \%$ yield

To a solution of 4,4-diallyl-6-bromoisochroman-3-one (1.2 g, $4 \mathrm{mmol})$, (4-nitrophenyl)boronic acid (0.8 g, $4.8 \mathrm{mmol})$ and $\mathrm{Pd}\left(\mathrm{PPh}_{3}\right)_{4}(462 \mathrm{mg}, 0.4 \mathrm{mmol})$ in THF (40 mL) and $\mathrm{MeOH}$ (6 mL), aqueous $\mathrm{K}_{2} \mathrm{CO}_{3}(2 \mathrm{M}, 10 \mathrm{~mL})$ were added under the argon. The mixture was degassed and heated at reflux with heating mantle for $30 \mathrm{~h}$. After cooled to ambient temperature, the reaction mixture was diluted by ethyl acetate $(3 \times 50 \mathrm{~mL})$, washed with brine, dried over $\mathrm{Na}_{2} \mathrm{SO}_{4}$ and concentrated. The residue was purified by column chromatography (ethyl acetate/petroleum ether, 1:5, v/v) to pure 4,4-diallyl-6-(4-nitrophenyl)isochroman-3-one (1k $1.1 \mathrm{~g}, 81 \%$ yield).

\section{4,4-diallyl-6-(4-nitrophenyl)isochroman-3-one (1k)}<smiles>C=CCC1(CC=C)C(=O)OCc2ccc(-c3ccc([N+](=O)[O-])cc3)cc21</smiles>

Light yellow solid, mp: $131-133{ }^{\circ} \mathrm{C}$, TLC $R_{f}=0.15(\mathrm{PE} / \mathrm{EA}=4: 1, \mathrm{v} / \mathrm{v}) .{ }^{1} \mathrm{H}$ NMR $(400 \mathrm{MHz}$, 
$\left.\mathrm{CDCl}_{3}\right) \delta 8.33(\mathrm{~d}, J=8.3 \mathrm{~Hz}, 2 \mathrm{H}), 7.73(\mathrm{~d}, J=8.6 \mathrm{~Hz}, 2 \mathrm{H}), 7.61-7.49(\mathrm{~m}, 2 \mathrm{H}), 7.30(\mathrm{~d}, J=$ $8.0 \mathrm{~Hz}, 1 \mathrm{H}), 5.68-5.50(\mathrm{~m}, 2 \mathrm{H}), 5.46(\mathrm{~s}, 2 \mathrm{H}), 5.20-4.97(\mathrm{~m}, 4 \mathrm{H}), 2.97$ (dd, $J=14.2,7.0 \mathrm{~Hz}$, $2 \mathrm{H}), 2.73(\mathrm{dd}, J=14.2,7.3 \mathrm{~Hz}, 2 \mathrm{H}) .{ }^{13} \mathrm{C} \mathrm{NMR}\left(101 \mathrm{MHz}, \mathrm{CDCl}_{3}\right) \delta 172.9,147.4,146.9,139.0$, 136.0, 132.5, 131.5, 127.9, 126.2, 125.8, 125.1, 124.3, 119.7, 69.1, 50.4, 43.1. ESI-HRMS calcd for $\left[\mathrm{C}_{21} \mathrm{H}_{19} \mathrm{NNaO}_{4}{ }^{+}, \mathrm{M}+\mathrm{Na}\right]^{+}:$372.1206, Found 372.1187.

\section{methyl 4-(4,4-diallyl-3-oxoisochroman-6-yl)benzoate (11)}

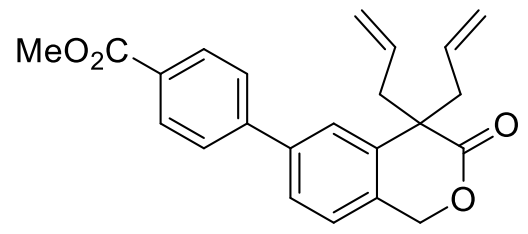

The 11 was prepared from 4,4-diallyl-6-bromoisochroman-3-one (1.2 g, $4 \mathrm{mmol}$ ) by Method C (1.4 g, 94\% yield).

Light yellow solid, mp: $104-106{ }^{\circ} \mathrm{C}$, TLC $R_{f}=0.22(\mathrm{PE} / \mathrm{EA}=4: 1, \mathrm{v} / \mathrm{v}) .{ }^{1} \mathrm{H}$ NMR $(400 \mathrm{MHz}$, $\left.\mathrm{CDCl}_{3}\right) \delta 8.14(\mathrm{~d}, J=8.4 \mathrm{~Hz}, 2 \mathrm{H}), 7.63(\mathrm{~d}, J=8.4 \mathrm{~Hz}, 2 \mathrm{H}), 7.56-7.50(\mathrm{~m}, 2 \mathrm{H}), 7.23(\mathrm{~d}, J=$ $8.4 \mathrm{~Hz}, 1 \mathrm{H}), 5.55$ (ddt, $J=17.2,10.1,7.2 \mathrm{~Hz}, 2 \mathrm{H}), 5.43$ (s, 2H), $5.14-5.03$ (m, 4H), 3.95 (s, 3H), $2.95(\mathrm{dd}, J=14.1,7.1 \mathrm{~Hz}, 2 \mathrm{H}), 2.71(\mathrm{dd}, J=14.1,7.3 \mathrm{~Hz}, 2 \mathrm{H}) .{ }^{13} \mathrm{C}$ NMR $(101 \mathrm{MHz}$, $\left.\mathrm{CDCl}_{3}\right) \delta 173.0,166.9,145.0,140.3,135.7,132.6,130.7,130.3,129.4,127.1,126.1,125.6$, 124.8, 119.6, 69.2, 52.3, 50.4, 43.2. ESI-HRMS calcd for $\left[\mathrm{C}_{23} \mathrm{H}_{22} \mathrm{NaO}_{4}{ }^{+}, \mathrm{M}+\mathrm{Na}\right]^{+}: 385.1410$, Found 385.1351.

\subsection{General procedure for the preparation of 1,6-dienes containing diol}

\section{Method D}

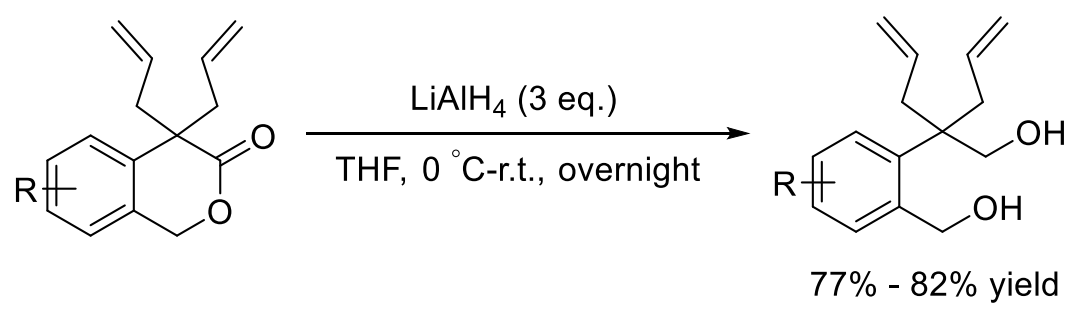

A two-neck $250 \mathrm{~mL}$ flask fitted with a magnetic stirring bar was charged with 4,4diallylisochroman-3-one (2.3 g, $10 \mathrm{mmol})$ under argon atmosphere. Dry THF (20 mL) was added, and the solution was cooled to $0{ }^{\circ} \mathrm{C}$. To this solution was added $\mathrm{LiAlH}_{4}(12 \mathrm{~mL}, 2.5 \mathrm{M}$ in THF, $30 \mathrm{mmol}$ ) using a slow addition pump over $10 \mathrm{~min}$. The solution was allowed to warm to room temperature overnight. After quenching with saturated $\mathrm{NH}_{4} \mathrm{Cl}$ under the $0{ }^{\circ} \mathrm{C}$, the reaction mixture was extracted with ethyl acetate $(3 \times 50 \mathrm{~mL})$. The organic layer was washed twice with brine, dried over $\mathrm{Na}_{2} \mathrm{SO}_{4}$ and concentrated. The residue was purified by column 
chromatography (ethyl acetate/petroleum ether, 1:2, v/v) to give pure 2-allyl-2-(2-(hydroxymethyl)phenyl)pent-4-en-1-ol (1p, $1.9 \mathrm{~g}, 82 \%$ yield).

\section{2-allyl-2-(2-(hydroxymethyl)phenyl)pent-4-en-1-ol (1p)}

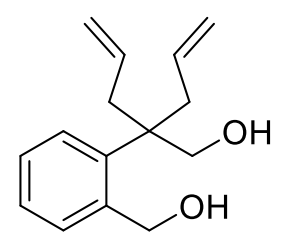

White solid, mp: 58-60 ${ }^{\circ} \mathrm{C}$, TLC $R_{f}=0.32(\mathrm{PE} / \mathrm{EA}=2: 1, \mathrm{v} / \mathrm{v}) .{ }^{1} \mathrm{H}$ NMR $(400 \mathrm{MHz}, \mathrm{DMSO}-$ $\left.d_{6}\right) \delta 7.51(\mathrm{~d}, J=7.3 \mathrm{~Hz}, 1 \mathrm{H}), 7.25-7.05(\mathrm{~m}, 3 \mathrm{H}), 5.45$ (ddt, $\left.J=17.3,10.1,7.2 \mathrm{~Hz}, 2 \mathrm{H}\right), 5.14$ $(\mathrm{t}, J=5.4 \mathrm{~Hz}, 1 \mathrm{H}), 4.96(\mathrm{ddt}, J=17.1,2.6,1.4 \mathrm{~Hz}, 2 \mathrm{H}), 4.86(\mathrm{dd}, J=10.2,2.5 \mathrm{~Hz}, 2 \mathrm{H}), 4.79-$ $4.61(\mathrm{~m}, 3 \mathrm{H}), 3.69(\mathrm{~d}, J=4.8 \mathrm{~Hz}, 2 \mathrm{H}), 3.32(\mathrm{~d}, J=1.0 \mathrm{~Hz}, 4 \mathrm{H}), 2.51(\mathrm{~s}, 1 \mathrm{H}) .{ }^{13} \mathrm{C}$ NMR $(101$ MHz, DMSO- $\left.d_{6}\right) \delta 140.8,140.8,135.4,129.6,127.6,126.4,125.8,116.9,64.2,61.7,46.4$. ESIHRMS calcd for $\left[\mathrm{C}_{15} \mathrm{H}_{20} \mathrm{NaO}_{2}^{+}, \mathrm{M}+\mathrm{Na}\right]^{+}:$255.1356, Found 255.1359.

\section{2-allyl-2-(2-(hydroxymethyl)-4-methoxyphenyl)pent-4-en-1-ol (1q)}

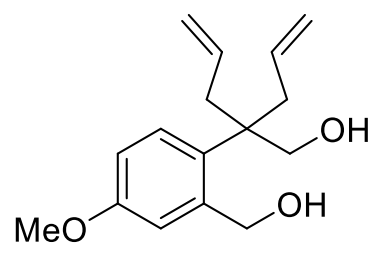

The 1q was prepared from 4,4-diallyl-7-methoxyisochroman-3-one (2.6 g, $10 \mathrm{mmol})$ by Method D (2.1 g, 82\% yield).

White solid, mp: $126-128{ }^{\circ} \mathrm{C}$, TLC $R_{f}=0.47(\mathrm{PE} / \mathrm{EA}=3: 2, \mathrm{v} / \mathrm{v}) .{ }^{1} \mathrm{H} \mathrm{NMR}\left(400 \mathrm{MHz}, \mathrm{CDCl}_{3}\right)$ $\delta 7.37-7.23(\mathrm{~m}, 2 \mathrm{H}), 7.02(\mathrm{~d}, J=3.0 \mathrm{~Hz}, 1 \mathrm{H}), 6.83(\mathrm{dd}, J=8.8,3.0 \mathrm{~Hz}, 1 \mathrm{H}), 5.67$ (ddt, $J=$ 17.2, 10.2, 7.2 Hz, 2H), 5.29 - 4.95 (m, 5H), 4.79 (s, 2H), 3.98 (s, 2H), 3.82 (s, 3H), 2.62 (dd, $J=14.3,6.9 \mathrm{~Hz}, 2 \mathrm{H}), 2.45(\mathrm{dd}, J=14.3,7.4 \mathrm{~Hz}, 2 \mathrm{H}) .{ }^{13} \mathrm{C} \mathrm{NMR}\left(101 \mathrm{MHz}, \mathrm{CDCl}_{3}\right) \delta 140.9$, 135.0, 132.1, 130.5, 118.2, 118.0, 113.1, 69.1, 65.2, 55.3, 47.5, 42.3. ESI-HRMS calcd for $\left[\mathrm{C}_{16} \mathrm{H}_{22} \mathrm{NaO}_{3}{ }^{+}, \mathrm{M}+\mathrm{Na}\right]^{+}:$285.1461, Found 285.1465.

\section{2-allyl-2-(2-(hydroxymethyl)-4-methylphenyl)pent-4-en-1-ol (1r)}

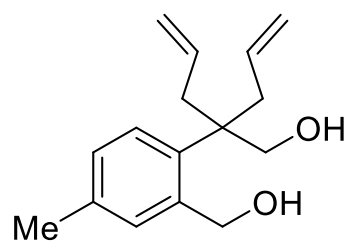

The 1r was prepared from 4,4-diallyl-7-methylisochroman-3-one (2.4 g, $10 \mathrm{mmol})$ by 
Method D (1.9 g, 77\% yield).

Colorless oil, TLC $R_{f}=0.58(\mathrm{PE} / \mathrm{EA}=3: 2, \mathrm{v} / \mathrm{v}) .{ }^{1} \mathrm{H}$ NMR $\left(400 \mathrm{MHz}, \mathrm{CDCl}_{3}\right) \delta 7.24(\mathrm{dd}, J$ $=8.2,5.7 \mathrm{~Hz}, 2 \mathrm{H}), 7.10(\mathrm{dd}, J=8.0,2.3 \mathrm{~Hz}, 1 \mathrm{H}), 5.67(\mathrm{ddt}, J=17.3,10.1,7.2 \mathrm{~Hz}, 2 \mathrm{H}), 5.16-$ 4.95 (m, 4H), 4.77 (s, 2H), 3.98 (d, $J=1.2 \mathrm{~Hz}, 2 \mathrm{H}), 2.63$ (ddt, $J=14.3,7.1,1.3 \mathrm{~Hz}, 2 \mathrm{H}), 2.47$ (ddt, $J=14.2,7.4,1.2 \mathrm{~Hz}, 2 \mathrm{H}), 2.33$ (s, 3H). ${ }^{13} \mathrm{C} \mathrm{NMR}\left(101 \mathrm{MHz}, \mathrm{CDCl}_{3}\right) \delta 139.2,137.3,136.8$, 135.0, 133.9, 129.3, 129.0, 118.1, 118.1, 69.0, 65.3, 47.8, 42.2, 42.1, 20.7. ESI-HRMS calcd for $\left[\mathrm{C}_{16} \mathrm{H}_{22} \mathrm{NaO}_{2}{ }^{+}, \mathrm{M}+\mathrm{Na}\right]^{+}:$269.1512, Found 269.1516 .

\section{2-allyl-2-(3-fluoro-2-(hydroxymethyl)phenyl)pent-4-en-1-ol (1s)}

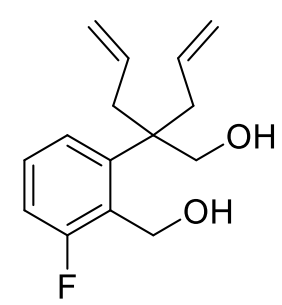

The 1s was prepared from 4,4-diallyl-8-fluoroisochroman-3-one (2.5 g, $10 \mathrm{mmol})$ by Method $\mathrm{D}(2.1 \mathrm{~g}, 82 \%$ yield $)$.

Colorless oil, TLC $R_{f}=0.58(\mathrm{PE} / \mathrm{EA}=1: 1, \mathrm{v} / \mathrm{v}) .{ }^{1} \mathrm{H}$ NMR $\left(400 \mathrm{MHz}, \mathrm{CDCl}_{3}\right) \delta 7.34-7.25$ (m, 1H), $7.17-7.11(\mathrm{~m}, 1 \mathrm{H}), 7.02$ (ddd, $J=9.5,8.1,1.1 \mathrm{~Hz}, 1 \mathrm{H}), 5.66$ (ddt, $J=17.2,10.1,7.2$ Hz, 2H), $5.24-4.99$ (m, 4H), 4.92 (d, $J=2.9 \mathrm{~Hz}, 2 \mathrm{H}), 4.03$ (s, 2H), 2.66 (ddt, $J=14.3,7.0$, $1.3 \mathrm{~Hz}, 2 \mathrm{H}), 2.49$ (ddt, $J=14.3,7.5,1.3 \mathrm{~Hz}, 2 \mathrm{H}) .{ }^{13} \mathrm{C} \mathrm{NMR}\left(101 \mathrm{MHz}, \mathrm{CDCl}_{3}\right) \delta 163.2(\mathrm{~d}, J=$ $244.5 \mathrm{~Hz}), 143.6$ (d, $J=1.9 \mathrm{~Hz}), 134.6,129.3$ (d, $J=10.0 \mathrm{~Hz}), 126.9$ (d, $J=13.6 \mathrm{~Hz}), 124.8$ $(\mathrm{d}, J=3.1 \mathrm{~Hz}), 118.4(\mathrm{~d}, J=2.4 \mathrm{~Hz}), 114.1$ (d, $J=23.8 \mathrm{~Hz}), 68.7,56.5$ (d, $J=9.6 \mathrm{~Hz}), 48.4$ (d, $J=1.9 \mathrm{~Hz}), 42.4 .{ }^{19} \mathrm{~F}$ NMR $\left(376 \mathrm{MHz}, \mathrm{CDCl}_{3}\right) \delta-116.4$. ESI-HRMS calcd for $\left[\mathrm{C}_{15} \mathrm{H}_{19} \mathrm{FNaO}_{2}{ }^{+}\right.$, $\mathrm{M}+\mathrm{Na}]^{+}:$273.1261, Found 273.1265.

\subsection{Procedure for the preparation of substrate $1 \mathrm{t}$}

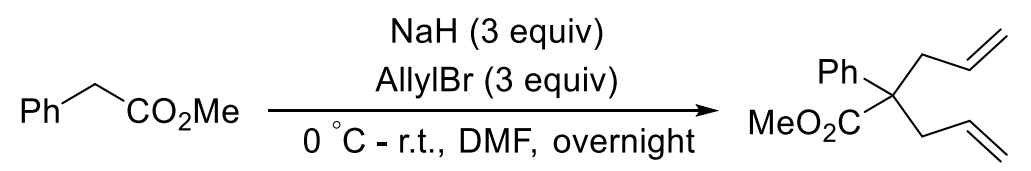

Sodium hydride $(1.5 \mathrm{~g}, 60 \mathrm{mmol})$ in dry DMF $(20 \mathrm{~mL})$ was cooled to $0{ }^{\circ} \mathrm{C}$. The methyl 2phenylacetate $(20 \mathrm{mmol})$ dissolved in $20 \mathrm{~mL}$ of dry DMF was added dropwise under the argon. The mixture was stirred at $0{ }^{\circ} \mathrm{C}$ for $1 \mathrm{~h}$, and then was allowed to warm to room temperature for $1 \mathrm{~h}$. After cooling to $0{ }^{\circ} \mathrm{C}$, allyl bromide $(7.2 \mathrm{~g}, 60 \mathrm{mmol})$ dissolved in dry DMF $(10 \mathrm{~mL})$ was added dropwise. The reaction mixture was allowed to warm to room temperature overnight, and then was cooled to $0{ }^{\circ} \mathrm{C}$, quenched by adding saturated $\mathrm{NH}_{4} \mathrm{Cl}$, and extracted by ethyl 
acetate. The combined organic phase was washed with saturated $\mathrm{NH}_{4} \mathrm{Cl}$, dried over $\mathrm{Na}_{2} \mathrm{SO}_{4}$, and concentrated. The residue was purified by chromatography (ethyl acetate/petroleum ether, 1:20, v/v) to give methyl 2-allyl-2-phenylpent-4-enoate (1t, $4.5 \mathrm{~g}, 98 \%$ yield).

methyl 2-allyl-2-phenylpent-4-enoate (1t)

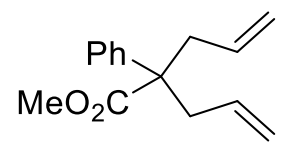

Colorless liquid, TLC $R_{f}=0.52(\mathrm{PE} / \mathrm{EA}=20: 1, \mathrm{v} / \mathrm{v}) .{ }^{1} \mathrm{H}$ NMR $\left(400 \mathrm{MHz}, \mathrm{CDCl}_{3}\right) \delta 7.39-$ $7.28(\mathrm{~m}, 2 \mathrm{H}), 7.28-7.16(\mathrm{~m}, 3 \mathrm{H}), 5.59-5.37(\mathrm{~m}, 2 \mathrm{H}), 5.07$ (ddd, $J=9.5,2.2,1.1 \mathrm{~Hz}, 2 \mathrm{H})$, $5.04-5.03(\mathrm{~m}, 2 \mathrm{H}), 3.64(\mathrm{~s}, 3 \mathrm{H}), 3.03-2.56(\mathrm{~m}, 4 \mathrm{H}) .{ }^{13} \mathrm{C} \mathrm{NMR}\left(101 \mathrm{MHz}, \mathrm{CDCl}_{3}\right) \delta 175.6$, $141.9,133.5,133.5,128.5,128.5,127.0,126.6,126.5,118.8,118.8,53.7,52.2,39.1,39.0$. ESIHRMS calcd for $\left[\mathrm{C}_{15} \mathrm{H}_{18} \mathrm{NaO}_{2}{ }^{+}, \mathrm{M}+\mathrm{Na}^{+}:\right.$253.1199, Found 253.1205.

\subsection{Procedure for the preparation of substrate $1 u$}
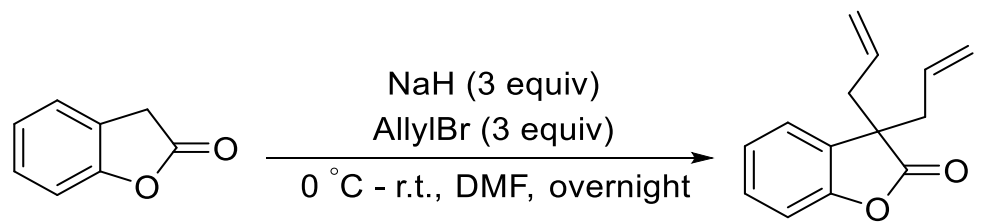

Sodium hydride $(0.72 \mathrm{~g}, 30 \mathrm{mmol})$ in dry DMF $(20 \mathrm{~mL})$ was cooled to $0{ }^{\circ} \mathrm{C}$. The benzofuran$2(3 \mathrm{H})$-one $(1.3 \mathrm{~g}, 10 \mathrm{mmol})$ dissolved in $20 \mathrm{~mL}$ of dry DMF was added dropwise under the argon. The mixture was stirred at $0{ }^{\circ} \mathrm{C}$ for $1 \mathrm{~h}$, and then was allowed to warm to room temperature for $1 \mathrm{~h}$. After cooling to $0{ }^{\circ} \mathrm{C}$, allyl bromide $(3.6 \mathrm{~g}, 30 \mathrm{mmol})$ dissolved in dry DMF $(10 \mathrm{~mL})$ was added dropwise. The reaction mixture was allowed to warm to room temperature overnight, and then was cooled to $0{ }^{\circ} \mathrm{C}$, quenched by adding saturated $\mathrm{NH}_{4} \mathrm{Cl}$, and extracted by ethyl acetate. The combined organic phase was washed with saturated $\mathrm{NH}_{4} \mathrm{Cl}$, dried over $\mathrm{Na}_{2} \mathrm{SO}_{4}$, and concentrated. The residue was purified by chromatography (ethyl acetate/petroleum ether, 1:4, v/v) to give 3,3-diallylbenzofuran-2(3H)-one (1u, $1.8 \mathrm{~g}, 85 \%$ yield).

\section{3,3-diallylbenzofuran-2(3H)-one (1u)}

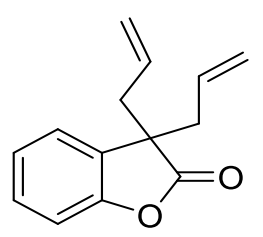


Colorless liquid, TLC $R_{f}=0.63(\mathrm{PE} / \mathrm{EA}=3: 1, \mathrm{v} / \mathrm{v}) .{ }^{1} \mathrm{H} \mathrm{NMR}\left(400 \mathrm{MHz}, \mathrm{CDCl}_{3}\right) \delta 7.30(\mathrm{tt}$, $J=7.7,2.3 \mathrm{~Hz}, 1 \mathrm{H}), 7.18(\mathrm{dtd}, J=14.8,7.5,1.4 \mathrm{~Hz}, 2 \mathrm{H}), 7.09$ (d, $J=7.9 \mathrm{~Hz}, 1 \mathrm{H}), 5.46$ (dddd, $J=17.3,12.0,8.7,5.3 \mathrm{~Hz}, 2 \mathrm{H}), 5.14-4.95(\mathrm{~m}, 4 \mathrm{H}), 2.70-2.55(\mathrm{~m}, 4 \mathrm{H}) .{ }^{13} \mathrm{C} \mathrm{NMR}(101 \mathrm{MHz}$, $\left.\mathrm{CDCl}_{3}\right) \delta 178.7,153.1,131.3,129.5,128.9,124.2,123.8,120.2,110.8,52.5,41.9 . E S I-H R M S$ calcd for $\left[\mathrm{C}_{14} \mathrm{H}_{14} \mathrm{NaO}_{2}{ }^{+}, \mathrm{M}+\mathrm{Na}\right]^{+}:$237.0886, Found 237.0891.

\subsection{Procedure for the preparation of substrate $1 \mathrm{v}$}
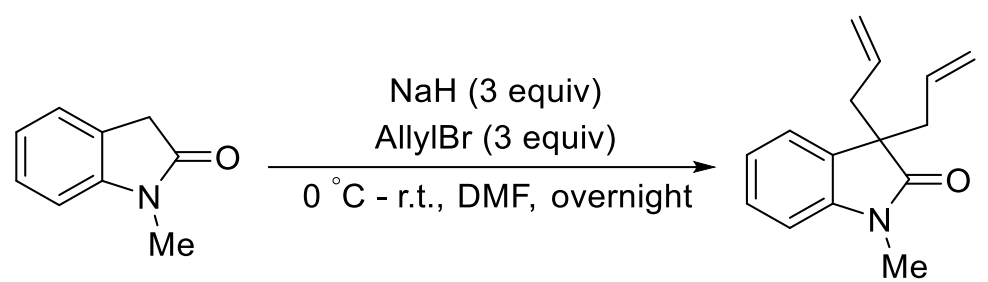

Sodium hydride $(0.72 \mathrm{~g}, 30 \mathrm{mmol})$ in dry DMF $(20 \mathrm{~mL})$ was cooled to $0{ }^{\circ} \mathrm{C}$. The 1 methylindolin-2-one (1.5 g, $10 \mathrm{mmol}$ ) dissolved in $20 \mathrm{~mL}$ of dry DMF was added dropwise under the argon. The mixture was stirred at $0{ }^{\circ} \mathrm{C}$ for $1 \mathrm{~h}$, and then was allowed to warm to room temperature for $1 \mathrm{~h}$. After cooling to $0{ }^{\circ} \mathrm{C}$, allyl bromide $(3.6 \mathrm{~g}, 30 \mathrm{mmol})$ dissolved in dry DMF $(10 \mathrm{~mL})$ was added dropwise. The reaction mixture was allowed to warm to room temperature overnight, and then was cooled to $0{ }^{\circ} \mathrm{C}$, quenched by adding saturated $\mathrm{NH}_{4} \mathrm{Cl}$, and extracted by ethyl acetate. The combined organic phase was washed with saturated $\mathrm{NH}_{4} \mathrm{Cl}$, dried over $\mathrm{Na}_{2} \mathrm{SO}_{4}$, and concentrated. The residue was purified by chromatography (ethyl acetate/petroleum ether, 1:6, v/v) to give 3,3-diallyl-1-methylindolin-2-one (1v, $1.8 \mathrm{~g}, 80 \%$ yield).

\section{3,3-diallyl-1-methylindolin-2-one (1v)}

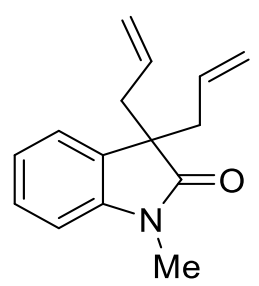

Colorless liquid, TLC $R_{f}=0.52(\mathrm{PE} / \mathrm{EA}=5: 1, \mathrm{v} / \mathrm{v}) .{ }^{1} \mathrm{H} \mathrm{NMR}\left(400 \mathrm{MHz}, \mathrm{CDCl}_{3}\right) \delta 7.31-7.23$ $(\mathrm{m}, 1 \mathrm{H}), 7.20(\mathrm{dd}, J=7.4,1.2 \mathrm{~Hz}, 1 \mathrm{H}), 7.06(\mathrm{td}, J=7.5,1.0 \mathrm{~Hz}, 1 \mathrm{H}), 6.82(\mathrm{~d}, J=7.6 \mathrm{~Hz}, 1 \mathrm{H})$, $5.41(\mathrm{ddt}, J=17.2,9.9,7.3 \mathrm{~Hz}, 2 \mathrm{H}), 5.07-4.84(\mathrm{~m}, 4 \mathrm{H}), 3.18(\mathrm{~s}, 3 \mathrm{H}), 2.64-2.50(\mathrm{~m}, 4 \mathrm{H}) .{ }^{13} \mathrm{C}$ NMR $\left(101 \mathrm{MHz}, \mathrm{CDCl}_{3}\right) \delta 179.0,143.9,132.4,131.4,128.0,123.5,122.4,118.9,107.9,52.8$, 41.3, 26.2. ESI-HRMS calcd for $\left[\mathrm{C}_{15} \mathrm{H}_{17} \mathrm{NNaO}^{+}, \mathrm{M}+\mathrm{Na}\right]^{+}:$250.1202, Found 250.1205. 


\section{Typical Procedure for Desymmetrizing Cyclization of 1,6-Dienes}

\section{Typical procedure for desymmetric cyclization of isochromanone-1,6-dienes}

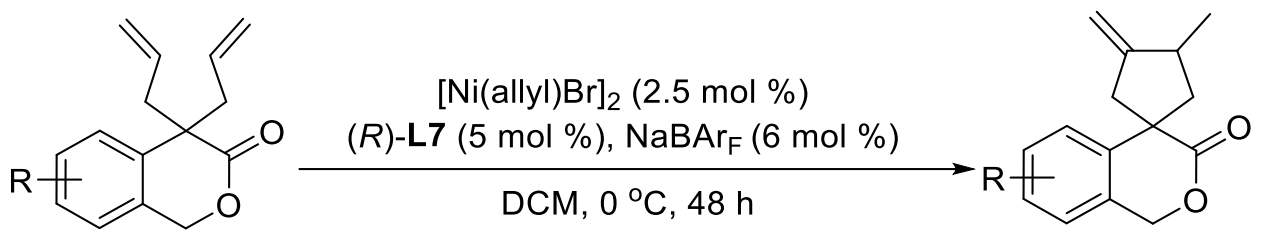

To the first Schlenk tube was added $[\mathrm{Ni}(\text { allyl }) \mathrm{Br}]_{2}(0.9 \mathrm{mg}, 0.0025 \mathrm{mmol}),(R)-\mathbf{L} 7(4.2 \mathrm{mg}$, $0.005 \mathrm{mmol}$ ) (except 2a which uses ligand $(S)-\mathbf{L} 7)$ and anhydrous dichloromethane $(1.5 \mathrm{~mL})$ under an argon atmosphere. The mixture was stirred at room temperature for $15 \mathrm{~min}$. To the second Schlenk tube was added $\mathrm{NaBAr}_{\mathrm{F}}(5.3 \mathrm{mg}, 0.006 \mathrm{mmol})$ and 1,6-diene $(0.1 \mathrm{mmol})$ under an argon atmosphere. The solution in the first Schlenk tube was transferred into the second Schlenk tube under an argon atmosphere. The resulting mixture was stirred at $0{ }^{\circ} \mathrm{C}$ for $48 \mathrm{~h}$. After removal of the solvent in vacuum, the residue was purified by column chromatography on silica gel (ethyl acetate/petroleum ether, 1:10, v/v) to afford products $\mathbf{2 a}-\mathbf{2 o}$.

\section{3-methyl-4-methylenespiro[cyclopentane-1,4'-isochroman]-3'-one (2a)}

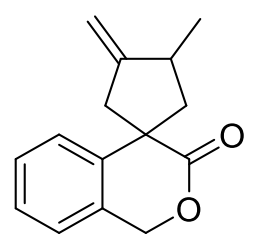

Colorless oil, $20.5 \mathrm{mg}, 90 \%$ yield, TLC $R_{f}=0.55(\mathrm{PE} / \mathrm{EA}=5: 1, \mathrm{v} / \mathrm{v}), \mathrm{dr}>20: 1,96 \%$ ee. HPLC condition: Chiralpak OJ-3 + AD-3 column $(25 \mathrm{~cm} \times 0.46 \mathrm{~cm}$ ID), hexane/2-propanol = 99:1, $0.5 \mathrm{~mL} / \mathrm{min}, 210 \mathrm{~nm} \mathrm{UV} \mathrm{detector,} t_{\mathrm{R}}=41.42 \mathrm{~min}$ (major) and $t_{\mathrm{R}}=46.31 \mathrm{~min}$ (minor). $[\alpha]_{\mathrm{D}}{ }^{25}-16.3\left(c 0.11, \mathrm{CHCl}_{3}\right) .{ }^{1} \mathrm{H} \mathrm{NMR}\left(400 \mathrm{MHz}, \mathrm{CDCl}_{3}\right) \delta 7.33(\mathrm{~d}, J=3.8 \mathrm{~Hz}, 2 \mathrm{H}), 7.30-$ $7.26(\mathrm{~m}, 1 \mathrm{H}), 7.21(\mathrm{~d}, J=7.0 \mathrm{~Hz}, 1 \mathrm{H}), 5.49(\mathrm{~d}, J=14.1 \mathrm{~Hz}, 1 \mathrm{H}), 5.27(\mathrm{~d}, J=14.2 \mathrm{~Hz}, 1 \mathrm{H})$, $5.11(\mathrm{q}, J=2.5 \mathrm{~Hz}, 1 \mathrm{H}), 4.95(\mathrm{q}, J=2.4 \mathrm{~Hz}, 1 \mathrm{H}), 3.59(\mathrm{dq}, J=17.5,2.8 \mathrm{~Hz}, 1 \mathrm{H}), 2.97-2.87$ (m, 1H), $2.62-2.54(\mathrm{~m}, 1 \mathrm{H}), 2.21(\mathrm{ddd}, J=11.9,7.1,2.0 \mathrm{~Hz}, 1 \mathrm{H}), 1.68(\mathrm{~d}, J=13.3 \mathrm{~Hz}, 1 \mathrm{H})$, $1.15(\mathrm{~d}, J=6.7 \mathrm{~Hz}, 3 \mathrm{H}) .{ }^{13} \mathrm{C}$ NMR $\left(101 \mathrm{MHz}, \mathrm{CDCl}_{3}\right) \delta 175.0,154.1,138.5,131.2,128.7$, 127.2, 125.4, 125. 0, 105.8, 69.2, 50.9, 43.9, 41.5, 37.1, 18.1. ESI-HRMS calcd for $\left[\mathrm{C}_{15} \mathrm{H}_{16} \mathrm{NaO}_{2}^{+}, \mathrm{M}+\mathrm{Na}\right]^{+}:$251.1043, Found 251.1046.

\section{3,7'-dimethyl-4-methylenespiro[cyclopentane-1,4'-isochroman]-3'-one (2b)}




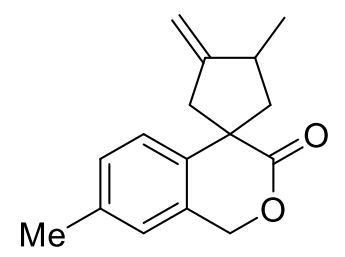

Colorless oil, $22.7 \mathrm{mg}, 94 \%$ yield, TLC $R_{f}=0.41(\mathrm{PE} / \mathrm{EA}=5: 1, \mathrm{v} / \mathrm{v}), \mathrm{dr}>20: 1,95 \%$ ee. HPLC condition: Chiralpak OJ-3 + AD-3 column $(25 \mathrm{~cm} \times 0.46 \mathrm{~cm}$ ID), hexane/2-propanol = 99:1, $0.5 \mathrm{~mL} / \mathrm{min}, 210 \mathrm{~nm} \mathrm{UV}$ detector, $t_{\mathrm{R}}=39.04 \mathrm{~min}$ (minor) and $t_{\mathrm{R}}=51.11 \mathrm{~min}$ (major). $[\alpha]_{\mathrm{D}}{ }^{25}+82.4\left(c 0.17, \mathrm{CHCl}_{3}\right) .{ }^{1} \mathrm{H}$ NMR $\left(400 \mathrm{MHz}, \mathrm{CDCl}_{3}\right) \delta 7.20(\mathrm{~d}, J=7.9 \mathrm{~Hz}, 1 \mathrm{H}), 7.16-$ $7.08(\mathrm{~m}, 1 \mathrm{H}), 7.01(\mathrm{~s}, 1 \mathrm{H}), 5.44(\mathrm{~d}, J=14.0 \mathrm{~Hz}, 1 \mathrm{H}), 5.23(\mathrm{~d}, J=14.0 \mathrm{~Hz}, 1 \mathrm{H}), 5.11(\mathrm{q}, J=2.4$ $\mathrm{Hz}, 1 \mathrm{H}), 4.95(\mathrm{q}, J=2.5 \mathrm{~Hz}, 1 \mathrm{H}), 2.97-2.82(\mathrm{~m}, 1 \mathrm{H}), 2.66-2.51(\mathrm{~m}, 1 \mathrm{H}), 2.35(\mathrm{~s}, 3 \mathrm{H}), 2.18$ (ddd, $J=12.1,7.1,2.2 \mathrm{~Hz}, 1 \mathrm{H}), 1.86(\mathrm{~s}, 1 \mathrm{H}), 1.14(\mathrm{~d}, J=6.7 \mathrm{~Hz}, 3 \mathrm{H}) .{ }^{13} \mathrm{C}$ NMR $(101 \mathrm{MHz}$, $\left.\mathrm{CDCl}_{3}\right) \delta 175.2,154.3,137.1,135.6,131.2,129.3,125.6,125.3,105.7,69.2,50.7,44.0,41.6$, 37.1, 21.1, 18.1. ESI-HRMS calcd for $\left[\mathrm{C}_{16} \mathrm{H}_{19} \mathrm{O}_{2}{ }^{+}, \mathrm{M}+\mathrm{H}\right]^{+}$: 243.1380, Found 243.1382.

\section{7'-chloro-3-methyl-4-methylenespiro[cyclopentane-1,4'-isochroman]-3'-one (2c)}

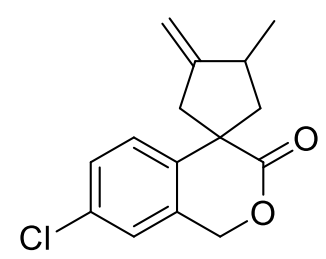

Colorless oil, $22.0 \mathrm{mg}$, 84\% yield, TLC $R_{f}=0.23(\mathrm{PE} / \mathrm{EA}=4: 1, \mathrm{v} / \mathrm{v})$, $\mathrm{dr}>20: 1,95 \%$ ee. HPLC condition: Chiralpak OJ-3 + AD-3 column $(25 \mathrm{~cm} \times 0.46 \mathrm{~cm}$ ID $)$, hexane/2-propanol = 99:1, $0.5 \mathrm{~mL} / \mathrm{min}, 210 \mathrm{~nm} \mathrm{UV}$ detector, $t_{\mathrm{R}}=47.14 \mathrm{~min}$ (minor) and $t_{\mathrm{R}}=53.20 \mathrm{~min}$ (major). $[\alpha]_{\mathrm{D}}{ }^{25}+48.8\left(c 0.16, \mathrm{CHCl}_{3}\right) .{ }^{1} \mathrm{H} \mathrm{NMR}\left(400 \mathrm{MHz}, \mathrm{CDCl}_{3}\right) \delta 7.32-7.23(\mathrm{~m}, 2 \mathrm{H}), 7.15(\mathrm{~d}, J=$ $7.9 \mathrm{~Hz}, 1 \mathrm{H}), 5.43$ (d, $J=14.3 \mathrm{~Hz}, 1 \mathrm{H}), 5.24$ (d, $J=14.3 \mathrm{~Hz}, 1 \mathrm{H}), 5.13$ (q, $J=2.4 \mathrm{~Hz}, 1 \mathrm{H}), 4.98$ $(\mathrm{q}, J=2.5 \mathrm{~Hz}, 1 \mathrm{H}), 3.58(\mathrm{dq}, J=17.5,2.8 \mathrm{~Hz}, 1 \mathrm{H}), 2.87(\mathrm{dq}, J=17.5,2.0 \mathrm{~Hz}, 1 \mathrm{H}), 2.77-2.43$ $(\mathrm{m}, 1 \mathrm{H}), 2.20(\mathrm{ddd}, J=12.3,7.2,2.2 \mathrm{~Hz}, 1 \mathrm{H}), 1.90(\mathrm{t}, J=12.0 \mathrm{~Hz}, 1 \mathrm{H}), 1.16(\mathrm{~d}, J=6.7 \mathrm{~Hz}$, $3 \mathrm{H}) .{ }^{13} \mathrm{C}$ NMR $\left(101 \mathrm{MHz}, \mathrm{CDCl}_{3}\right) \delta 174.2,153.3,140.7,134.9,129.8,127.5,126.4,125.6$, 106.5, 68.5, 50.9, 43.8, 41.5, 37.1, 18.2. ESI-HRMS calcd for $\left[\mathrm{C}_{15} \mathrm{H}_{15} \mathrm{ClNaO}_{2}{ }^{+}, \mathrm{M}+\mathrm{Na}^{+}\right.$: 285.0653 , Found 285.0658.

7'-methoxy-3-methyl-4-methylenespiro[cyclopentane-1,4'-isochroman]-3'-one (2d) 


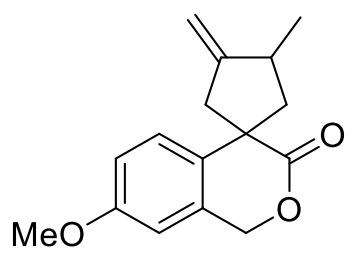

Colorless oil, $24.5 \mathrm{mg}$, 95\% yield, TLC $R_{f}=0.23(\mathrm{PE} / \mathrm{EA}=2: 1, \mathrm{v} / \mathrm{v}), \mathrm{dr}>20: 1,96 \%$ ee. HPLC condition: Chiralpak OJ-3 column $(25 \mathrm{~cm} \times 0.46 \mathrm{~cm} \mathrm{ID})$, hexane/2-propanol = 90:10, 1 $\mathrm{mL} / \mathrm{min}, 210 \mathrm{~nm}$ UV detector, $t_{\mathrm{R}}=16.18 \mathrm{~min}$ (minor) and $t_{\mathrm{R}}=20.33 \mathrm{~min}$ (major). $[\alpha]_{\mathrm{D}}{ }^{25}+41.1$ (c 0.18, $\left.\mathrm{CHCl}_{3}\right) .{ }^{1} \mathrm{H} \mathrm{NMR}\left(400 \mathrm{MHz}, \mathrm{CDCl}_{3}\right) \delta 7.23(\mathrm{~d}, J=8.6 \mathrm{~Hz}, 1 \mathrm{H}), 6.84(\mathrm{dd}, J=8.5,2.4$ $\mathrm{Hz}, 2 \mathrm{H}), 6.74(\mathrm{~d}, J=2.2 \mathrm{~Hz}, 1 \mathrm{H}), 5.44$ (d, $J=14.1 \mathrm{~Hz}, 1 \mathrm{H}), 5.21$ (d, $J=14.2 \mathrm{~Hz}, 1 \mathrm{H}), 5.10$ (q, $J=2.5 \mathrm{~Hz}, 1 \mathrm{H}), 4.94(\mathrm{q}, J=2.6 \mathrm{~Hz}, 1 \mathrm{H}), 3.80(\mathrm{~s}, 3 \mathrm{H}), 3.56(\mathrm{dd}, J=17.4,2.7 \mathrm{~Hz}, 1 \mathrm{H}), 2.87$ (d, $J=17.4 \mathrm{~Hz}, 1 \mathrm{H}), 2.72-2.50(\mathrm{~m}, 1 \mathrm{H}), 2.22-2.06(\mathrm{~m}, 1 \mathrm{H}), 1.95-1.76(\mathrm{~m}, 1 \mathrm{H}), 1.14(\mathrm{~d}, J=$ $6.7 \mathrm{~Hz}, 3 \mathrm{H}) .{ }^{13} \mathrm{C} \mathrm{NMR}\left(101 \mathrm{MHz}, \mathrm{CDCl}_{3}\right) \delta 175.1,158.8,154.3,132.6,130.6,126.7,113.9$, 110.7, 105.8, 69.1, 55.5, 50.4, 44.2, 41.8, 37. 1, 18.1. ESI-HRMS calcd for $\left[\mathrm{C}_{16} \mathrm{H}_{18} \mathrm{NaO}_{3}{ }^{+}, \mathrm{M}+\right.$ $\mathrm{Na}]^{+}:$281.1148, Found 281.1152.

\section{3,6'-dimethyl-4-methylenespiro[cyclopentane-1,4'-isochroman]-3'-one (2e)}<smiles>C=C1Cc2ccc(C)cc2C2(COC(=O)C(C)C2)C1</smiles>

White solid, $21.0 \mathrm{mg}, 87 \%$ yield, $\mathrm{mp}: 69-71^{\circ} \mathrm{C}$, TLC $R_{f}=0.23(\mathrm{PE} / \mathrm{EA}=4: 1, \mathrm{v} / \mathrm{v}), \mathrm{dr}>20: 1$, 94\% ee. HPLC condition: Chiralpak OJ-3 + AD-3 column $(25 \mathrm{~cm} \times 0.46 \mathrm{~cm}$ ID), hexane/2propanol $=99: 1,0.5 \mathrm{~mL} / \mathrm{min}, 210 \mathrm{~nm} \mathrm{UV} \mathrm{detector,} t_{\mathrm{R}}=38.30 \mathrm{~min}\left(\right.$ minor) and $t_{\mathrm{R}}=46.73 \mathrm{~min}$ (major). $[\alpha]_{\mathrm{D}}{ }^{25}+69.6\left(c 0.5, \mathrm{CHCl}_{3}\right) .{ }^{1} \mathrm{H} \mathrm{NMR}\left(400 \mathrm{MHz}, \mathrm{CDCl}_{3}\right) \delta 7.09$ (s, 4H), $5.44(\mathrm{~d}, J=$ $14.0 \mathrm{~Hz}, 1 \mathrm{H}), 5.23(\mathrm{~d}, J=14.0 \mathrm{~Hz}, 1 \mathrm{H}), 5.11(\mathrm{q}, J=2.4 \mathrm{~Hz}, 1 \mathrm{H}), 4.95(\mathrm{q}, J=2.5 \mathrm{~Hz}, 1 \mathrm{H}), 3.58$ $(\mathrm{dd}, J=17.5,2.7 \mathrm{~Hz}, 1 \mathrm{H}), 2.91(\mathrm{~d}, J=18.2 \mathrm{~Hz}, 1 \mathrm{H}), 2.61(\mathrm{dd}, J=11.4,2.5 \mathrm{~Hz}, 1 \mathrm{H}), 2.36$ (s, $3 \mathrm{H}), 2.20(\mathrm{dd}, J=11.4,8.7 \mathrm{~Hz}, 1 \mathrm{H}), 1.87(\mathrm{t}, J=11.9 \mathrm{~Hz}, 1 \mathrm{H}), 1.15(\mathrm{~d}, J=6.6 \mathrm{~Hz}, 3 \mathrm{H}) .{ }^{13} \mathrm{C}$ NMR (101 MHz, $\left.\mathrm{CDCl}_{3}\right) \delta 175.2,154.2,138.6,138.6,128.4,127.9,126.0,125.0,105.7,69.1$, 50.9, 43.9, 41.4, 37.2, 21.7, 18.0. ESI-HRMS calcd for $\left[\mathrm{C}_{16} \mathrm{H}_{18} \mathrm{NaO}_{2}{ }^{+}, \mathrm{M}+\mathrm{Na}\right]^{+}:$265.1199, Found 265.1205. 


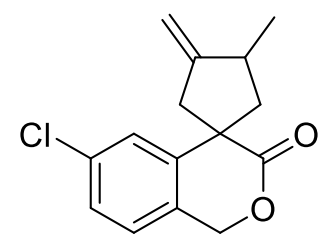

White solid, $23.0 \mathrm{mg}, 88 \%$ yield, $\mathrm{mp}: 56-58^{\circ} \mathrm{C}$, TLC $R_{f}=0.16(\mathrm{PE} / \mathrm{EA}=5: 1, \mathrm{v} / \mathrm{v}), \mathrm{dr}>20: 1$, 96\% ee. HPLC condition: Chiralpak OJ-3 + AD-3 column $(25 \mathrm{~cm} \times 0.46 \mathrm{~cm}$ ID), hexane/2propanol $=99: 1,0.5 \mathrm{~mL} / \mathrm{min}, 210 \mathrm{~nm} \mathrm{UV} \mathrm{detector,} t_{\mathrm{R}}=49.92 \mathrm{~min}$ (minor) and $t_{\mathrm{R}}=56.64 \mathrm{~min}$ (major). $[\alpha]_{\mathrm{D}}{ }^{25}+71.6\left(c\right.$ 0.5, $\left.\mathrm{CHCl}_{3}\right) .{ }^{1} \mathrm{H} \mathrm{NMR}\left(400 \mathrm{MHz}, \mathrm{CDCl}_{3}\right) \delta 7.27(\mathrm{dd}, J=10.0,2.1 \mathrm{~Hz}$, 4H), $7.15(\mathrm{~d}, J=7.9 \mathrm{~Hz}, 1 \mathrm{H}), 5.43(\mathrm{~d}, J=14.2 \mathrm{~Hz}, 1 \mathrm{H}), 5.25(\mathrm{~d}, J=14.2 \mathrm{~Hz}, 1 \mathrm{H}), 5.14(\mathrm{q}, J=$ $2.4 \mathrm{~Hz}, 1 \mathrm{H}), 4.98(\mathrm{q}, J=2.5 \mathrm{~Hz}, 1 \mathrm{H}), 3.59(\mathrm{dq}, J=17.5,2.8 \mathrm{~Hz}, 1 \mathrm{H}), 2.87$ (dq, $J=17.6,2.1$ $\mathrm{Hz}, 1 \mathrm{H}), 2.61$ (ddq, $J=14.2,9.6,2.7 \mathrm{~Hz}, 1 \mathrm{H}), 2.29-2.17(\mathrm{~m}, 2 \mathrm{H}), 1.89$ (t, $J=12.0 \mathrm{~Hz}, 1 \mathrm{H})$, $1.16(\mathrm{~d}, J=6.7 \mathrm{~Hz}, 3 \mathrm{H}) .{ }^{13} \mathrm{C}$ NMR $\left(101 \mathrm{MHz}, \mathrm{CDCl}_{3}\right) \delta 174.2,153.3,140.7,134.8,129.8$, 127.5, 126.4, 125.6, 106.5, 68.6, 50.9, 43.8, 41.5, 37.1, 18.2. ESI-HRMS calcd for $\left[\mathrm{C}_{15} \mathrm{H}_{15} \mathrm{ClNaO}_{2}^{+}, \mathrm{M}+\mathrm{Na}\right]^{+}:$285.0653, Found 285.0658 .

\section{6'-methoxy-3-methyl-4-methylenespiro[cyclopentane-1,4'-isochroman]-3'-one (2g)}

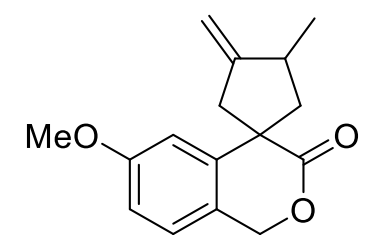

Colorless oil, $21.4 \mathrm{mg}, 83 \%$ yield, TLC $R_{f}=0.23(\mathrm{PE} / \mathrm{EA}=2: 1, \mathrm{v} / \mathrm{v}), \mathrm{dr}>20: 1,96 \%$ ee. HPLC condition: Chiralpak OJ-3 column ( $25 \mathrm{~cm} \times 0.46 \mathrm{~cm} \mathrm{ID)}$, hexane/2-propanol = 90:10, 1 $\mathrm{mL} / \mathrm{min}, 210 \mathrm{~nm}$ UV detector, $t_{\mathrm{R}}=19.19 \mathrm{~min}$ (minor) and $t_{\mathrm{R}}=53.12 \mathrm{~min}$ (major). $[\alpha]_{\mathrm{D}}{ }^{25}+48.2$ (c $\left.0.22, \mathrm{CHCl}_{3}\right) .{ }^{1} \mathrm{H} \mathrm{NMR}\left(400 \mathrm{MHz}, \mathrm{CDCl}_{3}\right) \delta 7.12(\mathrm{~d}, J=8.3 \mathrm{~Hz}, 1 \mathrm{H}), 6.89$ (d, $J=2.3 \mathrm{~Hz}$, $1 \mathrm{H}), 6.80(\mathrm{dd}, J=8.3,2.4 \mathrm{~Hz}, 1 \mathrm{H}), 5.43(\mathrm{~d}, J=13.8 \mathrm{~Hz}, 1 \mathrm{H}), 5.22(\mathrm{~d}, J=13.8 \mathrm{~Hz}, 1 \mathrm{H}), 5.12$ (q, $J=2.4 \mathrm{~Hz}, 1 \mathrm{H}), 4.95(\mathrm{q}, J=2.5 \mathrm{~Hz}, 1 \mathrm{H}), 3.80(\mathrm{~s}, 3 \mathrm{H}), 3.58(\mathrm{dd}, J=17.4,2.8 \mathrm{~Hz}, 1 \mathrm{H}), 2.89$ $(\mathrm{d}, J=17.5 \mathrm{~Hz}, 1 \mathrm{H}), 2.71-2.52(\mathrm{~m}, 1 \mathrm{H}), 2.30-2.11(\mathrm{~m}, 1 \mathrm{H}), 1.87(\mathrm{t}, J=11.9 \mathrm{~Hz}, 1 \mathrm{H}), 1.15$ $(\mathrm{d}, J=6.7 \mathrm{~Hz}, 3 \mathrm{H}) .{ }^{13} \mathrm{C} \mathrm{NMR}\left(101 \mathrm{MHz}, \mathrm{CDCl}_{3}\right) \delta 175.0,160.1,154.2,140.2,126.2,123.5$, 112.0, 111.7, 105.9, 68.8, 55.5, 51.2, 43.8, 41.5, 37.0, 18.2. ESI-HRMS calcd for $\left[\mathrm{C}_{16} \mathrm{H}_{18} \mathrm{NaO}_{3}{ }^{+}\right.$, $\mathrm{M}+\mathrm{Na}]^{+}:$281.1148, Found 281.1153.

6'-bromo-3-methyl-4-methylenespiro[cyclopentane-1,4'-isochroman]-3'-one (2h) 


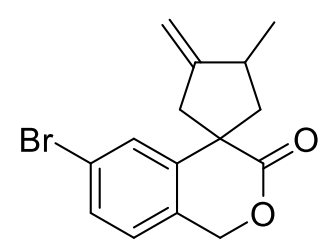

White solid, $25.0 \mathrm{mg}, 82 \%$ yield, $\mathrm{mp}: 70-72{ }^{\circ} \mathrm{C}$, TLC $R_{f}=0.25(\mathrm{PE} / \mathrm{EA}=5: 1, \mathrm{v} / \mathrm{v}), \mathrm{dr}>20: 1$, 96\% ee. HPLC condition: Chiralpak OJ-3 + AD-3 column $(25 \mathrm{~cm} \times 0.46 \mathrm{~cm}$ ID), hexane/2propanol $=99: 1,0.5 \mathrm{~mL} / \mathrm{min}, 210 \mathrm{~nm} \mathrm{UV} \mathrm{detector,} t_{\mathrm{R}}=51.26 \mathrm{~min}\left(\right.$ minor) and $t_{\mathrm{R}}=59.89 \mathrm{~min}$ (major). $[\alpha]_{\mathrm{D}}{ }^{25}+25.3\left(c 0.38, \mathrm{CHCl}_{3}\right) .{ }^{1} \mathrm{H} \mathrm{NMR}\left(400 \mathrm{MHz}, \mathrm{CDCl}_{3}\right) \delta 7.43(\mathrm{~d}, J=6.8 \mathrm{~Hz}, 2 \mathrm{H})$, $7.09(\mathrm{~d}, J=8.4 \mathrm{~Hz}, 1 \mathrm{H}), 5.41(\mathrm{~d}, J=14.3 \mathrm{~Hz}, 1 \mathrm{H}), 5.23(\mathrm{~d}, J=14.3 \mathrm{~Hz}, 1 \mathrm{H}), 5.13(\mathrm{q}, J=2.4$ $\mathrm{Hz}, 1 \mathrm{H}), 4.98$ (q, $J=2.5 \mathrm{~Hz}, 1 \mathrm{H}), 3.58(\mathrm{dd}, J=17.6,2.9 \mathrm{~Hz}, 1 \mathrm{H}), 2.87$ (d, $J=17.5 \mathrm{~Hz}, 1 \mathrm{H})$, $2.60(\mathrm{dtd}, J=12.0,6.0,2.9 \mathrm{~Hz}, 1 \mathrm{H}), 2.30-2.07(\mathrm{~m}, 2 \mathrm{H}), 1.89$ (t, $J=12.0 \mathrm{~Hz}, 1 \mathrm{H}), 1.16$ (d, $J$ $=6.6 \mathrm{~Hz}, 3 \mathrm{H}) .{ }^{13} \mathrm{C} \mathrm{NMR}\left(101 \mathrm{MHz}, \mathrm{CDCl}_{3}\right) \delta 174.2,153.3,141.0,130.4,130.3,128.5,126.7$, 122.9, 106.5, 68.6, 50.9, 43.8, 41.5, 37.1, 18.2. ESI-HRMS calcd for $\left[\mathrm{C}_{15} \mathrm{H}_{15} \mathrm{BrNaO}_{2}{ }^{+}, \mathrm{M}+\right.$ $\mathrm{Na}]^{+}:$329.0148, Found 329.0153.

\section{6'-fluoro-3-methyl-4-methylenespiro[cyclopentane-1,4'-isochroman]-3'-one (2i)}

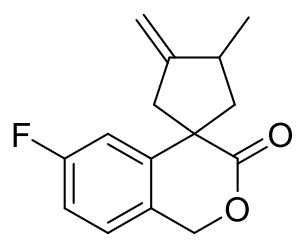

Colorless oil, $23.1 \mathrm{mg}$, 94\% yield, TLC $R_{f}=0.30(\mathrm{PE} / \mathrm{EA}=5: 1, \mathrm{v} / \mathrm{v}), \mathrm{dr}>20: 1,96 \%$ ee. HPLC condition: Chiralpak OJ-3 + AD-3 column $(25 \mathrm{~cm} \times 0.46 \mathrm{~cm}$ ID), hexane/2-propanol = 99:1, $0.5 \mathrm{~mL} / \mathrm{min}, 210 \mathrm{~nm} \mathrm{UV} \mathrm{detector,} t_{\mathrm{R}}=46.86 \mathrm{~min}$ (minor) and $t_{\mathrm{R}}=53.87 \mathrm{~min}$ (major). $[\alpha]_{\mathrm{D}}{ }^{25}+65.0\left(c 0.5, \mathrm{CHCl}_{3}\right) .{ }^{1} \mathrm{H} \mathrm{NMR}\left(400 \mathrm{MHz}, \mathrm{CDCl}_{3}\right) \delta 7.18(\mathrm{dd}, J=8.2,5.5 \mathrm{~Hz}, 1 \mathrm{H}), 7.05$ $(\mathrm{dd}, J=9.8,2.4 \mathrm{~Hz}, 1 \mathrm{H}), 6.98(\mathrm{td}, J=8.4,2.4 \mathrm{~Hz}, 1 \mathrm{H}), 5.44$ (d, $J=14.5 \mathrm{~Hz}, 1 \mathrm{H}), 5.25$ (d, $J=$ $14.1 \mathrm{~Hz}, 1 \mathrm{H}), 5.14(\mathrm{q}, J=2.4 \mathrm{~Hz}, 1 \mathrm{H}), 4.98(\mathrm{q}, J=2.5 \mathrm{~Hz}, 1 \mathrm{H}), 3.59(\mathrm{dd}, J=17.5,2.8 \mathrm{~Hz}, 1 \mathrm{H})$, $2.86(\mathrm{dd}, J=17.5,2.1 \mathrm{~Hz}, 1 \mathrm{H}), 1.89(\mathrm{t}, J=11.9 \mathrm{~Hz}, 1 \mathrm{H}), 1.16(\mathrm{~d}, J=6.7 \mathrm{~Hz}, 3 \mathrm{H}) .{ }^{13} \mathrm{C} \mathrm{NMR}$ $\left(101 \mathrm{MHz}, \mathrm{CDCl}_{3}\right) \delta 174.3,163.0(\mathrm{~d}, J=246.9 \mathrm{~Hz}), 153.4,141.1,127.1(\mathrm{~d}, J=3.1 \mathrm{~Hz}), 126.7$ $(\mathrm{d}, J=8.7 \mathrm{~Hz}), 114.2(\mathrm{~d}, J=21.8 \mathrm{~Hz}), 112.9(\mathrm{~d}, J=23.6 \mathrm{~Hz}), 106.4,68.6,51.0(\mathrm{~d}, J=1.8 \mathrm{~Hz})$, 43.7, 41.5, 37.0, 18.2. ${ }^{19} \mathrm{~F}$ NMR (376 $\left.\mathrm{MHz}, \mathrm{CDCl}_{3}\right) \delta-112.3$. ESI-HRMS calcd for $\left[\mathrm{C}_{15} \mathrm{H}_{15} \mathrm{FNaO}_{2}^{+}, \mathrm{M}+\mathrm{Na}\right]^{+}:$269.0948, Found 269.0953.

\section{3-methyl-4-methylene-6' -(trifluoromethyl)spiro[cyclopentane-1,4'-isochroman]-3'-one} (2j) 


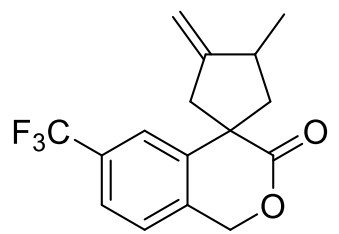

White solid, $24.0 \mathrm{mg}, 81 \%$ yield, $\mathrm{mp}: 85-88^{\circ} \mathrm{C}$, TLC $R_{f}=0.19(\mathrm{PE} / \mathrm{EA}=5: 1, \mathrm{v} / \mathrm{v}), \mathrm{dr}>20: 1$, 92\% ee. HPLC condition: Chiralpak OJ-3 + AD-3 column $(25 \mathrm{~cm} \times 0.46 \mathrm{~cm}$ ID), hexane/2propanol $=99: 1,0.5 \mathrm{~mL} / \mathrm{min}, 210 \mathrm{~nm} \mathrm{UV} \mathrm{detector,} t_{\mathrm{R}}=40.40 \mathrm{~min}$ (minor) and $t_{\mathrm{R}}=43.26 \mathrm{~min}$ (major). $[\alpha]_{\mathrm{D}}{ }^{25}+44.4\left(c 0.36, \mathrm{CHCl}_{3}\right) .{ }^{1} \mathrm{H} \mathrm{NMR}\left(400 \mathrm{MHz}, \mathrm{CDCl}_{3}\right) \delta 7.65-7.47(\mathrm{~m}, 2 \mathrm{H}), 7.35$ $(\mathrm{d}, J=8.2 \mathrm{~Hz}, 1 \mathrm{H}), 5.62-5.26(\mathrm{~m}, 2 \mathrm{H}), 5.51(\mathrm{~d}, J=14.6 \mathrm{~Hz}, 1 \mathrm{H}), 5.34(\mathrm{~d}, J=14.6 \mathrm{~Hz}, 1 \mathrm{H})$, $5.16(\mathrm{q}, J=2.4 \mathrm{~Hz}, 1 \mathrm{H}), 5.00(\mathrm{q}, J=2.5 \mathrm{~Hz}, 1 \mathrm{H}), 3.62(\mathrm{dq}, J=17.5,2.8 \mathrm{~Hz}, 1 \mathrm{H}), 2.91(\mathrm{dq}, J=$ 17.5, 2.0 Hz, 1H), $2.65-2.43(\mathrm{~m}, 2 \mathrm{H}), 2.22(\mathrm{ddd}, J=12.0,7.2,1.8 \mathrm{~Hz}, 2 \mathrm{H}), 1.94(\mathrm{t}, J=12.0$ $\mathrm{Hz}, 1 \mathrm{H}), 1.18(\mathrm{~d}, J=6.6 \mathrm{~Hz}, 3 \mathrm{H}) .{ }^{13} \mathrm{C} \mathrm{NMR}\left(101 \mathrm{MHz}, \mathrm{CDCl}_{3}\right) \delta 174.0,153.2,139.9,135.1$, $131.3(\mathrm{~d}, J=32.6 \mathrm{~Hz}), 125.7,124.4(\mathrm{q}, J=3.7 \mathrm{~Hz}), 123.9(\mathrm{~d}, J=272.6 \mathrm{~Hz}), 122.3(\mathrm{q}, J=3.7$ $\mathrm{Hz}), 106.7,68.6,51.0,43.9,41.6,37.1,18.3 .{ }^{19} \mathrm{~F} \mathrm{NMR}\left(376 \mathrm{MHz}, \mathrm{CDCl}_{3}\right) \delta-67.3$. ESI-HRMS calcd for $\left[\mathrm{C}_{16} \mathrm{H}_{15} \mathrm{~F}_{3} \mathrm{NaO}_{2}{ }^{+}, \mathrm{M}+\mathrm{Na}\right]^{+}:$319.0916, Found 319.0922 .

\section{3-methyl-4-methylene-6' -(4-nitrophenyl)spiro[cyclopentane-1,4'-isochroman]-3'-one (2k)}

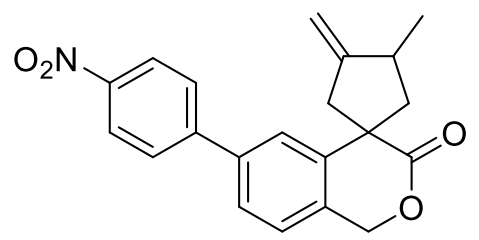

White solid, $30.0 \mathrm{mg}, 86 \%$ yield, mp: $145-147^{\circ} \mathrm{C}$, TLC $R_{f}=0.32(\mathrm{PE} / \mathrm{EA}=3: 1$, v/v), $\mathrm{dr}>$ 20:1, 86\% ee. HPLC condition: Chiralpak AD-3 column $(25 \mathrm{~cm} \times 0.46 \mathrm{~cm}$ ID), hexane/2propanol $=90: 10,1.0 \mathrm{~mL} / \mathrm{min}, 210 \mathrm{~nm} \mathrm{UV}$ detector, $t_{\mathrm{R}}=17.29 \mathrm{~min}$ (major) and $t_{\mathrm{R}}=19.00 \mathrm{~min}$ (minor). $[\alpha]_{\mathrm{D}}{ }^{25}+26.3\left(c 0.19, \mathrm{CHCl}_{3}\right) .{ }^{1} \mathrm{H} \mathrm{NMR}\left(400 \mathrm{MHz}, \mathrm{CDCl}_{3}\right) \delta 8.31(\mathrm{~d}, J=8.8 \mathrm{~Hz}, 2 \mathrm{H})$, $7.69(\mathrm{~d}, J=8.8 \mathrm{~Hz}, 2 \mathrm{H}), 7.56(\mathrm{~d}, J=7.3 \mathrm{~Hz}, 2 \mathrm{H}), 7.36(\mathrm{~d}, J=7.7 \mathrm{~Hz}, 1 \mathrm{H}), 5.55$ (d, $J=14.4$ $\mathrm{Hz}, 1 \mathrm{H}), 5.35(\mathrm{~d}, J=14.4 \mathrm{~Hz}, 1 \mathrm{H}), 5.17$ (q, $J=2.3 \mathrm{~Hz}, 1 \mathrm{H}), 5.00(\mathrm{q}, J=2.5 \mathrm{~Hz}, 1 \mathrm{H}), 3.66(\mathrm{dq}$, $J=17.5,2.8 \mathrm{~Hz}, 1 \mathrm{H}), 2.99(\mathrm{dd}, J=17.5,2.2 \mathrm{~Hz}, 1 \mathrm{H}), 2.75-2.51(\mathrm{~m}, 1 \mathrm{H}), 2.28(\mathrm{ddd}, J=12.2$, 7.1, $2.2 \mathrm{~Hz}, 1 \mathrm{H}), 1.95(\mathrm{t}, J=11.9 \mathrm{~Hz}, 1 \mathrm{H}), 1.19(\mathrm{~d}, J=6.7 \mathrm{~Hz}, 3 \mathrm{H}) \cdot{ }^{13} \mathrm{C} \mathrm{NMR}\left(101 \mathrm{MHz}, \mathrm{CDCl}_{3}\right)$ $\delta 174.5,153.9,147.4,146.9,139.7,139.4,132.0,128.0,126.4,126.0,124.4,124.3,106.3,68.8$, 51.1, 43.9, 41.6, 37.2, 18.3. ESI-HRMS calcd for $\left[\mathrm{C}_{21} \mathrm{H}_{19} \mathrm{NNaO}_{4}{ }^{+}, \mathrm{M}+\mathrm{Na}\right]^{+}:$372.1206, Found 372.1211 .

methyl 4-(3-methyl-4-methylene-3'-oxospiro[cyclopentane-1,4'-isochroman]-6'- 
yl)benzoate (2l)

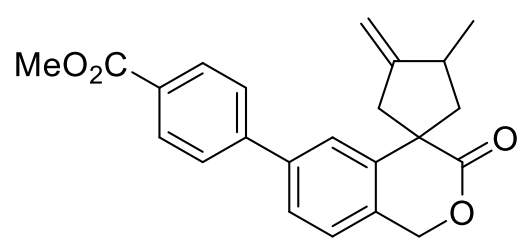

Light yellow solid, $32.6 \mathrm{mg}$, $92 \%$ yield, mp: $129-132{ }^{\circ} \mathrm{C}$, TLC $R_{f}=0.30(\mathrm{PE} / \mathrm{EA}=4: 1$, v/v), dr $>20: 1,87 \%$ ee. HPLC condition: Chiralpak AD-3 column $(25 \mathrm{~cm} \times 0.46 \mathrm{~cm}$ ID), hexane/2propanol $=80: 20,0.8 \mathrm{~mL} / \mathrm{min}, 210 \mathrm{~nm} \mathrm{UV}$ detector, $t_{\mathrm{R}}=9.62 \mathrm{~min}$ (major) and $t_{\mathrm{R}}=12.22 \mathrm{~min}$ (minor). $[\alpha]_{\mathrm{D}}{ }^{25}+47.9\left(c 0.38, \mathrm{CHCl}_{3}\right) .{ }^{1} \mathrm{H} \mathrm{NMR}\left(400 \mathrm{MHz}, \mathrm{CDCl}_{3}\right) \delta 8.11(\mathrm{~d}, J=8.0 \mathrm{~Hz}, 3 \mathrm{H})$, $7.74-7.44(\mathrm{~m}, 5 \mathrm{H}), 7.31(\mathrm{~d}, J=7.7 \mathrm{~Hz}, 1 \mathrm{H}), 55.53(\mathrm{~d}, J=14.3 \mathrm{~Hz}, 1 \mathrm{H}), 5.32(\mathrm{~d}, J=14.3 \mathrm{~Hz}$, $1 \mathrm{H}), 5.15(\mathrm{~d}, J=2.8 \mathrm{~Hz}, 1 \mathrm{H}), 4.98(\mathrm{q}, J=2.4 \mathrm{~Hz}, 1 \mathrm{H}), 3.94(\mathrm{~s}, 3 \mathrm{H}), 3.63(\mathrm{dt}, J=17.7,2.9 \mathrm{~Hz}$, 1H), $2.98(\mathrm{~d}, J=17.5 \mathrm{~Hz}, 1 \mathrm{H}), 2.74-2.53(\mathrm{~m}, 1 \mathrm{H}), 2.27$ (ddd, $J=12.2,7.1,2.0 \mathrm{~Hz}, 1 \mathrm{H}), 1.92$ $(\mathrm{t}, J=11.9 \mathrm{~Hz}, 1 \mathrm{H}), 1.17(\mathrm{~d}, J=6.6 \mathrm{~Hz}, 3 \mathrm{H}) .{ }^{13} \mathrm{C} \mathrm{NMR}\left(101 \mathrm{MHz}, \mathrm{CDCl}_{3}\right) \delta 174.6,166.8$, 153.9, 144.8, 140.5, 139.3, 131.1, 130.3, 129.4, 127.1, 126.1, 125.7, 124.1, 106.1, 68.8, 52.2, 51.0, 43.9, 41.5, 37.1, 18.2. ESI-HRMS calcd for $\left[\mathrm{C}_{23} \mathrm{H}_{22} \mathrm{NaO}_{4}{ }^{+}, \mathrm{M}+\mathrm{H}\right]^{+}:$385.1410, Found 385.1415 .

\section{8'-methyl-4-methylenespiro[cyclopentane-1,4'-isochroman]-3'-one (2m)}<smiles>C=C1CC2(CC1C)C(=O)OCc1c(C)cccc12</smiles>

Colorless oil, $22.0 \mathrm{mg}, 91 \%$ yield, TLC $R_{f}=0.33(\mathrm{PE} / \mathrm{EA}=4: 1, \mathrm{v} / \mathrm{v}), \mathrm{dr}>20: 1,94 \%$ ee. HPLC condition: Chiralpak AS-3 column $(25 \mathrm{~cm} \times 0.46 \mathrm{~cm} \mathrm{ID})$, hexane/2-propanol = 90:10, 1 $\mathrm{mL} / \min , 210 \mathrm{~nm} \mathrm{UV}$ detector, $t_{\mathrm{R}}=49.56 \mathrm{~min}$ (major) and $t_{\mathrm{R}}=64.97 \mathrm{~min}$ (minor). $[\alpha]_{\mathrm{D}}{ }^{25}+71.2$ $\left(c\right.$ 0.2, $\left.\mathrm{CHCl}_{3}\right) .{ }^{1} \mathrm{H} \mathrm{NMR}\left(400 \mathrm{MHz}, \mathrm{CDCl}_{3}\right) \delta 7.24-7.14(\mathrm{~m}, 2 \mathrm{H}), 7.11(\mathrm{~d}, J=7.3 \mathrm{~Hz}, 1 \mathrm{H})$, $5.41(\mathrm{q}, J=14.6 \mathrm{~Hz}, 2 \mathrm{H}), 5.10(\mathrm{q}, J=2.4 \mathrm{~Hz}, 1 \mathrm{H}), 4.93(\mathrm{q}, J=2.5 \mathrm{~Hz}, 1 \mathrm{H}), 3.59$ (dq, $J=17.5$, $2.7 \mathrm{~Hz}, 1 \mathrm{H}), 2.92(\mathrm{dq}, J=17.4,2.0 \mathrm{~Hz}, 1 \mathrm{H}), 2.64-2.45$ (m, 0H), 2.31 (s, 3H), 2.20 (ddd, $J=$ 12.0, 7.2, $2.2 \mathrm{~Hz}, 1 \mathrm{H}), 1.86(\mathrm{t}, J=11.9 \mathrm{~Hz}, 1 \mathrm{H}), 1.13(\mathrm{~d}, J=6.7 \mathrm{~Hz}, 3 \mathrm{H}) .{ }^{13} \mathrm{C} \mathrm{NMR}(101 \mathrm{MHz}$, $\left.\mathrm{CDCl}_{3}\right) \delta 175.2,154.3,138.5,133.5,129.6,129.1,128.4,123.2,105.7,66.4,50.7,44.1,41.6$, 37.1, 18.6, 18.0. ESI-HRMS calcd for $\left[\mathrm{C}_{16} \mathrm{H}_{19} \mathrm{O}_{2}{ }^{+}, \mathrm{M}+\mathrm{H}\right]^{+}:$: 243.1380, Found 243.1383.

\section{8'-fluoro-3-methyl-4-methylenespiro[cyclopentane-1,4'-isochroman]-3'-one (2n)}




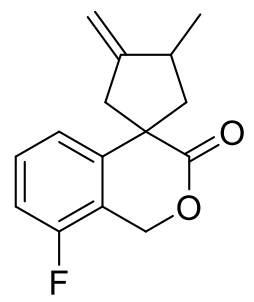

White solid, $21.9 \mathrm{mg}, 89 \%$ yield, $\mathrm{mp}: 85-88^{\circ} \mathrm{C}$, TLC $R_{f}=0.52(\mathrm{PE} / \mathrm{EA}=5: 1, \mathrm{v} / \mathrm{v}), \mathrm{dr}>20: 1$, 91\% ee. HPLC condition: Chiralpak OJ-3 + AD-3 column $(25 \mathrm{~cm} \times 0.46 \mathrm{~cm}$ ID), hexane/2propanol $=99: 1,0.5 \mathrm{~mL} / \mathrm{min}, 210 \mathrm{~nm} \mathrm{UV}$ detector, $t_{\mathrm{R}}=33.64 \mathrm{~min}\left(\right.$ minor) and $t_{\mathrm{R}}=34.91 \mathrm{~min}$ (major). $[\alpha]_{\mathrm{D}}{ }^{25}+67.0\left(c 0.23, \mathrm{CHCl}_{3}\right) .{ }^{1} \mathrm{H} \mathrm{NMR}\left(400 \mathrm{MHz}, \mathrm{CDCl}_{3}\right) \delta 7.37-7.21(\mathrm{~m}, 1 \mathrm{H}), 7.12$ $(\mathrm{d}, J=7.8 \mathrm{~Hz}, 1 \mathrm{H}), 7.01(\mathrm{t}, J=8.7 \mathrm{~Hz}, 1 \mathrm{H}), 5.55(\mathrm{~d}, J=14.7 \mathrm{~Hz}, 1 \mathrm{H}), 5.37(\mathrm{~d}, J=14.7 \mathrm{~Hz}$, 1H), $5.11(\mathrm{q}, J=2.4 \mathrm{~Hz}, 1 \mathrm{H}), 4.95(\mathrm{q}, J=2.6 \mathrm{~Hz}, 1 \mathrm{H}), 3.60(\mathrm{dq}, J=17.5,2.9 \mathrm{~Hz}, 1 \mathrm{H}), 2.91(\mathrm{~d}$, $J=17.4 \mathrm{~Hz}, 1 \mathrm{H}), 2.34-2.00(\mathrm{~m}, 1 \mathrm{H}), 1.89(\mathrm{t}, J=11.9 \mathrm{~Hz}, 1 \mathrm{H}), 1.15(\mathrm{~d}, J=6.6 \mathrm{~Hz}, 3 \mathrm{H}) .{ }^{13} \mathrm{C}$ $\operatorname{NMR}\left(101 \mathrm{MHz}, \mathrm{CDCl}_{3}\right) \delta 174.4,158.1(\mathrm{~d}, J=247.6 \mathrm{~Hz}), 153.8,141.3(\mathrm{~d}, J=2.7 \mathrm{~Hz}), 130.0$ (d, $J=8.5 \mathrm{~Hz}), 121.0(\mathrm{~d}, J=3.2 \mathrm{~Hz}), 118.6$ (d, $J=16.3 \mathrm{~Hz}), 114.0(\mathrm{~d}, J=20.6 \mathrm{~Hz}), 106.1,62.8$ $(\mathrm{d}, J=4.9 \mathrm{~Hz}), 50.9$ (d, $J=1.9 \mathrm{~Hz}), 44.2,41.6,37.1,18.1 .{ }^{19} \mathrm{~F}$ NMR $\left(377 \mathrm{MHz}, \mathrm{CDCl}_{3}\right) \delta-$ 120.5. ESI-HRMS calcd for $\left[\mathrm{C}_{15} \mathrm{H}_{15} \mathrm{FNaO}_{2}{ }^{+}, \mathrm{M}+\mathrm{Na}\right]^{+}: 269.0948$, Found 269.0953.

\section{3-methyl-4-methylene-5' H,7'H-spiro[cyclopentane-1,8'-[1,3]dioxolo[4,5-g]isochromen]- 7'-one (2o)}

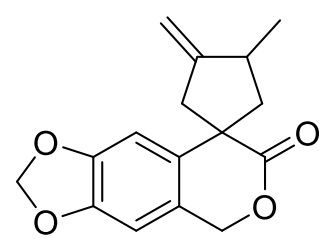

Colorless oil, $25.0 \mathrm{mg}$, 92\% yield, TLC $R_{f}=0.4(\mathrm{PE} / \mathrm{EA}=4: 1, \mathrm{v} / \mathrm{v}), \mathrm{dr}>20: 1,88 \%$ ee, HPLC condition: Chiralpak AD-3 column ( $25 \mathrm{~cm} \times 0.46 \mathrm{~cm} \mathrm{ID)}$, hexane/2-propanol = 95:5, $1.0 \mathrm{~mL} / \mathrm{min}, 220 \mathrm{~nm} \mathrm{UV}$ detector, $t_{\mathrm{R}}=15.18 \mathrm{~min}$ (major) and $t_{\mathrm{R}}=17.03 \mathrm{~min}$ (minor), $[\alpha]_{\mathrm{D}}{ }^{25}$ $+46.5\left(c\right.$ 0.16, $\left.\mathrm{CHCl}_{3}\right) .{ }^{1} \mathrm{H} \mathrm{NMR}\left(400 \mathrm{MHz}, \mathrm{CDCl}_{3}\right) \delta 6.83(\mathrm{~s}, 1 \mathrm{H}), 6.66(\mathrm{~s}, 1 \mathrm{H}), 6.08-5.88(\mathrm{~m}$, 2H), $5.48-4.89(\mathrm{~m}, 4 \mathrm{H}), 3.56(\mathrm{dd}, J=17.5,2.9 \mathrm{~Hz}, 1 \mathrm{H}), 2.83(\mathrm{dq}, J=17.5,2.0 \mathrm{~Hz}, 1 \mathrm{H}), 2.60$ (dtt, $J=12.0,7.4,2.6 \mathrm{~Hz}, 1 \mathrm{H}), 2.18(\mathrm{ddd}, J=12.0,7.2,2.3 \mathrm{~Hz}, 1 \mathrm{H}), 1.84(\mathrm{t}, J=11.9 \mathrm{~Hz}, 1 \mathrm{H})$, $1.15(\mathrm{~d}, J=6.7 \mathrm{~Hz}, 3 \mathrm{H}) .{ }^{13} \mathrm{C} \mathrm{NMR}\left(101 \mathrm{MHz}, \mathrm{CDCl}_{3}\right) \delta 174.9,154.0,148.2,146.8,132.3$, 124.8, 106.4, 106.1, 105.6, 101.6, 68.9, 50.8, 44.0, 41.9, 37.1, 18.3. ESI-HRMS calcd for $\left[\mathrm{C}_{16} \mathrm{H}_{16} \mathrm{NaO}_{4}{ }^{+}, \mathrm{M}+\mathrm{Na}\right]^{+}:$295.0941, Found 295.0945.

In a $1 \mathrm{mmol}$ scale experiment, $223.0 \mathrm{mg}, 82 \%$ yield, $\mathrm{dr}>20: 1,86 \%$ ee of $2 \mathrm{o}$ was obtained.

\section{Typical procedure for desymmetric cyclization of 1,6-dienes containing diol}




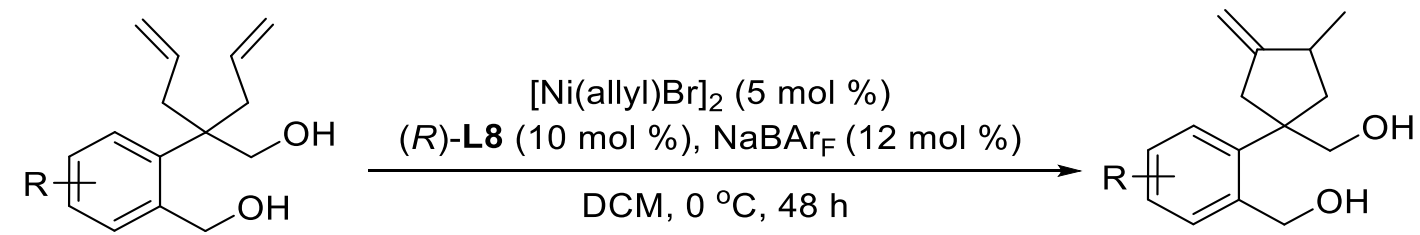

To the first Schlenk tube was added $[\mathrm{Ni}(\text { allyl }) \mathrm{Br}]_{2}(1.8 \mathrm{mg}, 0.005 \mathrm{mmol}),(R)-\mathbf{L 8}(10.2 \mathrm{mg}$, $0.010 \mathrm{mmol})$, and anhydrous dichloromethane $(1.5 \mathrm{~mL})$ under an argon atmosphere. The mixture was stirred at room temperature for $15 \mathrm{~min}$. To the second Schlenk tube was added $\operatorname{NaBAr}_{\mathrm{F}}(10.6 \mathrm{mg}, 0.012 \mathrm{mmol})$ and 1,6-diene $(0.1 \mathrm{mmol})$ under an argon atmosphere. The solution in the first Schlenk tube was transferred into the second Schlenk tube. The resulting mixture was stirred at $0{ }^{\circ} \mathrm{C}$ for $48 \mathrm{~h}$. After removal of the solvent, the residue was purified by column chromatography on silica gel (ethyl acetate/petroleum ether, 1:3, v/v) to afford product.

\section{2-allyl-2-(2-(hydroxymethyl)phenyl)pent-4-en-1-ol (2p)}

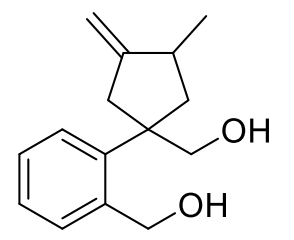

Colorless oil, $20.2 \mathrm{mg}$, 87\% yield, TLC $R_{f}=0.37(\mathrm{PE} / \mathrm{EA}=3: 1, \mathrm{v} / \mathrm{v}), \mathrm{dr}=10: 1,89 \%$ ee. HPLC condition: Chiralpak AS-3 column $(25 \mathrm{~cm} \times 0.46 \mathrm{~cm} \mathrm{ID})$, hexane/2-propanol = 96:4, 1 $\mathrm{mL} / \mathrm{min}, 210 \mathrm{~nm}$ UV detector, $t_{\mathrm{R}}=32.64 \mathrm{~min}$ (major) and $t_{\mathrm{R}}=34.15 \min$ (minor). $[\alpha]_{\mathrm{D}}{ }^{25}+52.0$ (c $\left.0.20, \mathrm{CHCl}_{3}\right) .{ }^{1} \mathrm{H}$ NMR $\left(400 \mathrm{MHz}, \mathrm{CDCl}_{3}\right) \delta 7.37(\mathrm{dd}, J=6.5,1.7 \mathrm{~Hz}, 1 \mathrm{H}), 7.31-7.27(\mathrm{~m}$, $2 \mathrm{H}), 7.26-7.21(\mathrm{~m}, 1 \mathrm{H}), 5.01(\mathrm{tt}, J=2.4,1.1 \mathrm{~Hz}, 1 \mathrm{H}), 4.82(\mathrm{tt}, J=2.4,1.1 \mathrm{~Hz}, 1 \mathrm{H}), 4.71(\mathrm{q}$, $J=12.1,10.6 \mathrm{~Hz}, 2 \mathrm{H}), 3.63-3.48(\mathrm{~m}, 2 \mathrm{H}), 3.07-2.78(\mathrm{~m}, 2 \mathrm{H}), 2.67-2.56(\mathrm{~m}, 1 \mathrm{H}), 2.45(\mathrm{dd}$, $J=13.0,10.6 \mathrm{~Hz}, 1 \mathrm{H}), 1.83-1.75(\mathrm{~m}, 1 \mathrm{H}), 1.71(\mathrm{~s}, 1 \mathrm{H}), 1.15(\mathrm{~d}, J=6.8 \mathrm{~Hz}, 3 \mathrm{H}) .{ }^{13} \mathrm{C} \mathrm{NMR}$ $\left(101 \mathrm{MHz}, \mathrm{CDCl}_{3}\right) \delta 155.4,144.1,139.5,131.7,129.3,128.2,127.1,105.5,70.5,64.4,51.5$, 44.7, 44.1, 35.5, 19.6. ESI-HRMS calcd for $\left[\mathrm{C}_{15} \mathrm{H}_{20} \mathrm{NaO}_{2}^{+}, \mathrm{M}+\mathrm{Na}\right]^{+}:$255.1356, Found 255.1360 .

\section{(2-(1-(hydroxymethyl)-3-methyl-4-methylenecyclopentyl)-5-methoxyphenyl)methanol} (2q)

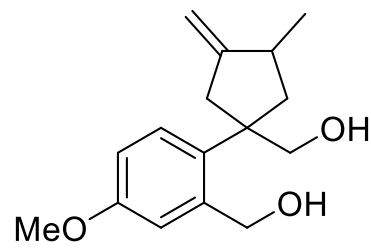

Colorless oil, $21.0 \mathrm{mg}, 80 \%$ yield, TLC $R_{f}=0.32(\mathrm{PE} / \mathrm{EA}=1: 1, \mathrm{v} / \mathrm{v}), \mathrm{dr}=10: 1,89 \%$ ee. 
HPLC condition: Chiralpak OJ-H column $(25 \mathrm{~cm} \times 0.46 \mathrm{~cm}$ ID), hexane/2-propanol = 98.2:1.8, $1 \mathrm{~mL} / \mathrm{min}, 210 \mathrm{~nm}$ UV detector, $t_{\mathrm{R}}=69.95 \mathrm{~min}$ (major) and $t_{\mathrm{R}}=77.79 \min$ (minor). $[\alpha]_{\mathrm{D}}{ }^{25}$ +65.6 (c 0.125, $\left.\mathrm{CHCl}_{3}\right) .{ }^{1} \mathrm{H} \mathrm{NMR}\left(400 \mathrm{MHz}, \mathrm{CDCl}_{3}\right) \delta 7.20(\mathrm{~d}, J=8.7 \mathrm{~Hz}, 1 \mathrm{H}), 6.96(\mathrm{~d}, J=$ $3.0 \mathrm{~Hz}, 1 \mathrm{H}), 6.80(\mathrm{dd}, J=8.7,2.9 \mathrm{~Hz}, 1 \mathrm{H}), 5.03-4.98(\mathrm{~m}, 1 \mathrm{H}), 4.83-4.79(\mathrm{~m}, 1 \mathrm{H}), 4.75-$ $4.62(\mathrm{~m}, 2 \mathrm{H}), 3.80(\mathrm{~s}, 3 \mathrm{H}), 3.53(\mathrm{~d}, J=3.6 \mathrm{~Hz}, 2 \mathrm{H}), 2.94-2.79(\mathrm{~m}, 2 \mathrm{H}), 2.68-2.52(\mathrm{~m}, 2 \mathrm{H})$, $2.41(\mathrm{dd}, J=13.1,10.5 \mathrm{~Hz}, 1 \mathrm{H}), 1.81-1.69(\mathrm{~m}, 1 \mathrm{H}), 1.14(\mathrm{~d}, J=6.8 \mathrm{~Hz}, 3 \mathrm{H}) .{ }^{13} \mathrm{C} \mathrm{NMR}(101$ $\left.\mathrm{MHz}, \mathrm{CDCl}_{3}\right) \delta 158.3,155.6,141.0,135.6,130.5,116.5,113.2,105.5,70.4,64.2,55.4,50.8$, 44.9, 44.2, 35.47, 19.6. ESI-HRMS calcd for $\left[\mathrm{C}_{16} \mathrm{H}_{22} \mathrm{NaO}_{3}{ }^{+}, \mathrm{M}+\mathrm{Na}\right]^{+}:$285.1461, Found 285.1465 .

(2-(1-(hydroxymethyl)-3-methyl-4-methylenecyclopentyl)-5-methylphenyl)methanol (2r)

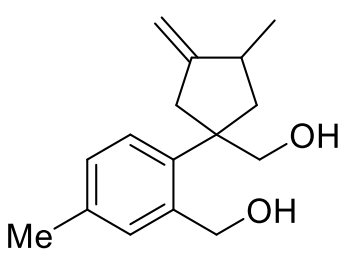

Colorless oil, $20.2 \mathrm{mg}, 82 \%$ yield, TLC $R_{f}=0.40(\mathrm{PE} / \mathrm{EA}=1: 1, \mathrm{v} / \mathrm{v}), \mathrm{dr}=10: 1,87 \%$ ee. HPLC condition: Chiralpak AS-3 column $(25 \mathrm{~cm} \times 0.46 \mathrm{~cm} \mathrm{ID})$, hexane/2-propanol = 97:3, $0.75 \mathrm{~mL} / \mathrm{min}, 210 \mathrm{~nm} \mathrm{UV}$ detector, $t_{\mathrm{R}}=37.17 \mathrm{~min}$ (minor) and $t_{\mathrm{R}}=38.54 \mathrm{~min}$ (major). $[\alpha]_{\mathrm{D}}{ }^{25}$ +51.0 (c 0.1, $\left.\mathrm{CHCl}_{3}\right) .{ }^{1} \mathrm{H} \mathrm{NMR}\left(400 \mathrm{MHz}, \mathrm{CDCl}_{3}\right) \delta 7.25-7.15(\mathrm{~m}, 2 \mathrm{H}), 7.10(\mathrm{dd}, J=8.1,2.1$ $\mathrm{Hz}, 1 \mathrm{H}), 4.94(\mathrm{~s}, 1 \mathrm{H}), 4.75$ (s, 1H), 4.65 (q, J = 11.9, $9.6 \mathrm{~Hz}, 2 \mathrm{H}), 3.65-3.52$ (m, 2H), 2.99 $2.80(\mathrm{~m}, 2 \mathrm{H}), 2.63(\mathrm{dt}, J=17.1,8.2 \mathrm{~Hz}, 1 \mathrm{H}), 2.46(\mathrm{dd}, J=13.0,10.5 \mathrm{~Hz}, 1 \mathrm{H}), 2.33(\mathrm{~s}, 3 \mathrm{H})$, $1.85-1.73(\mathrm{~m}, 1 \mathrm{H}), 1.15(\mathrm{~d}, J=6.8 \mathrm{~Hz}, 3 \mathrm{H}) .{ }^{13} \mathrm{C} \mathrm{NMR}\left(101 \mathrm{MHz}, \mathrm{CDCl}_{3}\right) \delta 155.6,140.8$, 139.4, 136.8, 132.5, 129.3, 129.0, 105.4, 70. 7, 64.4, 51.2, 44.8, 44.2, 35.5, 20.8, 19.6. ESIHRMS calcd for $\left[\mathrm{C}_{16} \mathrm{H}_{22} \mathrm{NaO}_{2}{ }^{+}, \mathrm{M}+\mathrm{Na}\right]^{+}:$269.1512, Found 269.1517.

(1-(3-fluoro-2-(hydroxymethyl)phenyl)-3-methyl-4-methylenecyclopentyl)methanol (2s)<smiles>C=C1CC(CO)(c2cccc(F)c2CO)CC1C</smiles>

Colorless oil, $23.0 \mathrm{mg}$, 92\% yield, TLC $R_{f}=0.55(\mathrm{PE} / \mathrm{EA}=1: 1, \mathrm{v} / \mathrm{v}), \mathrm{dr}=10: 1,82 \%$ ee. HPLC condition: Chiralpak AS-3 column $(25 \mathrm{~cm} \times 0.46 \mathrm{~cm} \mathrm{ID})$, hexane/2-propanol = 99:1, $0.75 \mathrm{~mL} / \mathrm{min}, 210 \mathrm{~nm} \mathrm{UV}$ detector, $t_{\mathrm{R}}=27.37 \mathrm{~min}$ (major) and $t_{\mathrm{R}}=29.74 \mathrm{~min}$ (minor). $[\alpha]_{\mathrm{D}}{ }^{25}$ 
+34.7 $\left(c\right.$ 0.19, $\left.\mathrm{CHCl}_{3}\right) .{ }^{1} \mathrm{H}$ NMR $\left(400 \mathrm{MHz}, \mathrm{CDCl}_{3}\right) \delta 7.32-7.25(\mathrm{~m}, 1 \mathrm{H}), 7.13(\mathrm{~d}, J=7.9 \mathrm{~Hz}$, 1H), $7.02(\mathrm{t}, J=9.0 \mathrm{~Hz}, 1 \mathrm{H}), 5.02(\mathrm{~s}, 1 \mathrm{H}), 4.85(\mathrm{~s}, 3 \mathrm{H}), 3.64(\mathrm{q}, J=10.7 \mathrm{~Hz}, 2 \mathrm{H}), 2.92(\mathrm{~s}, 2 \mathrm{H})$, $2.84-2.54(\mathrm{~m}, 1 \mathrm{H}), 2.50(\mathrm{dd}, J=12.9,10.2 \mathrm{~Hz}, 1 \mathrm{H}), 1.82(\mathrm{dd}, J=12.6,7.6 \mathrm{~Hz}, 1 \mathrm{H}), 1.20$ (dd, $J=16.6,6.4 \mathrm{~Hz}, 4 \mathrm{H}) .{ }^{13} \mathrm{C} \mathrm{NMR}\left(101 \mathrm{MHz}, \mathrm{CDCl}_{3}\right) \delta 163.0(\mathrm{~d}, J=244.8 \mathrm{~Hz}), 155.1,147.1$, $129.3(\mathrm{~d}, J=10.5 \mathrm{~Hz}), 127.1(\mathrm{~d}, J=13.6 \mathrm{~Hz}), 124.7(\mathrm{~d}, J=3.0 \mathrm{~Hz}), 113.9(\mathrm{~d}, J=7.9 \mathrm{~Hz})$, 105.4, 70.9, $56.1(\mathrm{~d}, J=9.4 \mathrm{~Hz}), 51.7,44.7(\mathrm{~d}, J=67.7 \mathrm{~Hz}), 35.8,25.5,19.2 .{ }^{19} \mathrm{~F}$ NMR $(376$ $\left.\mathrm{MHz}, \mathrm{CDCl}_{3}\right) \delta-117.1$. ESI-HRMS calcd for $\left[\mathrm{C}_{15} \mathrm{H}_{19} \mathrm{FNaO}_{2}{ }^{+}, \mathrm{M}+\mathrm{Na}\right]^{+}:$273.1261, Found 273.1266 .

\section{Typical procedure for desymmetrizing cyclization of 1,6-diene 1t}

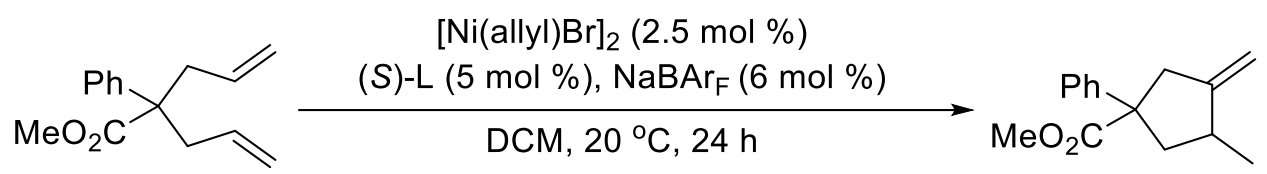

To the first Schlenk tube was added $[\mathrm{Ni}(\text { allyl }) \mathrm{Br}]_{2}(0.9 \mathrm{mg}, 0.0025 \mathrm{mmol})$, ligand $(S)$-L9 (4.2 $\mathrm{mg}, 0.005 \mathrm{mmol})$, and anhydrous dichloromethane $(1.5 \mathrm{~mL})$ under an argon atmosphere. The mixture was stirred at room temperature for $15 \mathrm{~min}$. To the second Schlenk tube was added $\operatorname{NaBAr}_{F}(5.3 \mathrm{mg}, 0.006 \mathrm{mmol})$ and 1,6-diene $(0.1 \mathrm{mmol})$ under an argon atmosphere. The solution in the first Schlenk tube was transferred into the second Schlenk tube. The resulting mixture was stirred at $20^{\circ} \mathrm{C}$ for $24 \mathrm{~h}$. After removal of the solvent, the residue was purified by column chromatography on silica gel (ethyl acetate/petroleum ether, 1:20-1:10, v/v) to afford product.

methyl 3-methyl-4-methylene-1-phenylcyclopentane-1-carboxylate (2t)

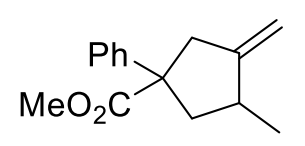

Colorless oil, $22.1 \mathrm{mg}, 96 \%$ yield, TLC $R_{f}=0.54(\mathrm{PE} / \mathrm{EA}=15: 1, \mathrm{v} / \mathrm{v}), \mathrm{dr}=3: 1,76 \%$ ee (major), 97\% ee (minor). HPLC condition: Chiralpak ID-3 + OJ-3 column $(25 \mathrm{~cm} \times 0.46 \mathrm{~cm}$ ID), hexane/2-propanol = 98:2, $0.5 \mathrm{~mL} / \mathrm{min}, 210 \mathrm{~nm} \mathrm{UV}$ detector, $\left[t_{\mathrm{R} 1}=31.60 \mathrm{~min}\right.$ (major) and $t_{\mathrm{R} 1}=39.18 \mathrm{~min}$ (minor)] (minor); [ $t_{\mathrm{R} 2}=32.69 \mathrm{~min}$ (major) and $t_{\mathrm{R} 2}=35.74 \mathrm{~min}$ (minor) $]$ (major). $[\alpha]_{\mathrm{D}}{ }^{25}-49.0\left(c 0.2, \mathrm{CHCl}_{3}\right) .{ }^{1} \mathrm{H} \mathrm{NMR}\left(400 \mathrm{MHz}, \mathrm{CDCl}_{3}\right) \delta 7.33(\mathrm{dt}, J=15.1,7.7 \mathrm{~Hz}, 4 \mathrm{H}), 7.23$ $(\mathrm{d}, J=2.5 \mathrm{~Hz}, 1 \mathrm{H}), 5.02-4.71(\mathrm{~m}, 2 \mathrm{H}), 3.61(\mathrm{~s}, 3 \mathrm{H}), 3.20-2.93(\mathrm{~m}, 2 \mathrm{H}), 2.78(\mathrm{t}, J=7.2 \mathrm{~Hz}$, $1 \mathrm{H}), 2.52-2.33(\mathrm{~m}, 1 \mathrm{H}), 2.18(\mathrm{dd}, J=12.0,8.8 \mathrm{~Hz}, 1 \mathrm{H}), 1.10(\mathrm{~d}, J=6.5 \mathrm{~Hz}, 1 \mathrm{H}) .{ }^{13} \mathrm{C}$ NMR $\left(101 \mathrm{MHz}, \mathrm{CDCl}_{3}\right) \delta 154.6 / 154.5,133.5,128.5 / 128.5,127.0 / 126.8,126.6 / 126.5,118.8$, 105.9/105.7, 55.8/53.7, 44.7, 43.3/43.0, 39.1, 37.3/36.2, 19.2/19.0. ESI-HRMS calcd for 


\section{Typical procedure for desymmetrizing cyclization of 1,6-diene $2 u$}
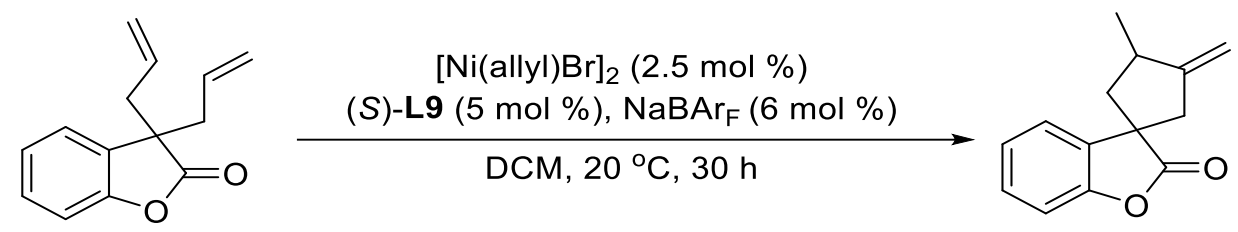

To the first Schlenk tube was added $[\mathrm{Ni}(\text { allyl }) \mathrm{Br}]_{2}(0.9 \mathrm{mg}, 0.0025 \mathrm{mmol})$, ligand $(S)$-L9 (4.2 $\mathrm{mg}, 0.005 \mathrm{mmol})$, and anhydrous dichloromethane $(1.5 \mathrm{~mL})$ under an argon atmosphere. The mixture was stirred at room temperature for $15 \mathrm{~min}$. To the second Schlenk tube was added $\operatorname{NaBAr}_{\mathrm{F}}(5.3 \mathrm{mg}, 0.006 \mathrm{mmol})$ and 3,3-diallylbenzofuran-2(3H)-one $(0.1 \mathrm{mmol})$ under an argon atmosphere. The solution in the first Schlenk tube was transferred into the second Schlenk tube. The resulting mixture was stirred at $20{ }^{\circ} \mathrm{C}$ for $30 \mathrm{~h}$. After removal of the solvent, the residue was purified by column chromatography on silica gel (ethyl acetate/petroleum ether, 1:5, v/v) to afford $2 \mathbf{u}(20.0 \mathrm{mg}, 95 \%$ yield $)$.

\section{3'-methyl-4'-methylene-2H-spiro[benzofuran-3,1'-cyclopentan]-2-one (2u)}

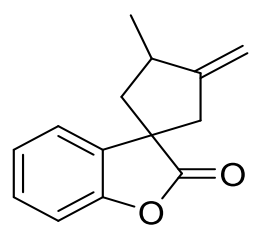

Colorless liquid, TLC $R_{f}=0.61(\mathrm{PE} / \mathrm{EA}=3: 1, \mathrm{v} / \mathrm{v})$. HPLC condition: Chiralpak OJ-3 + AD3 column $(25 \mathrm{~cm} \times 0.46 \mathrm{~cm} \mathrm{ID})$, hexane/2-propanol = 98:2, $0.5 \mathrm{~mL} / \mathrm{min}, 210 \mathrm{~nm} \mathrm{UV}$ detector, $\left[t_{\mathrm{R} 1}=23.46 \mathrm{~min}\right.$ (major) and $t_{\mathrm{R} 1}=24.41 \mathrm{~min}$ (minor) $]$ (major); $\left[t_{\mathrm{R} 2}=26.83 \mathrm{~min}\right.$ (minor) and $t_{\mathrm{R} 2}$ $=28.29 \mathrm{~min}$ (major)] (minor). $[\alpha]_{\mathrm{D}}{ }^{25}-20.4\left(c 0.2, \mathrm{CHCl}_{3}\right) .{ }^{1} \mathrm{H} \mathrm{NMR}\left(400 \mathrm{MHz}, \mathrm{CDCl}_{3}\right) \delta 7.33$ $-7.21(\mathrm{~m}, 2 \mathrm{H}), 7.17-7.07(\mathrm{~m}, 2 \mathrm{H}), 5.05(\mathrm{dq}, J=14.0,2.4 \mathrm{~Hz}, 2 \mathrm{H}), 3.11(\mathrm{dq}, J=16.2,2.6 \mathrm{~Hz}$, 1H), 3.01 (dddd, $J=13.9,9.3,5.5,2.2 \mathrm{~Hz}, 1 \mathrm{H}), 2.71-2.57$ (m, 1H), 2.17 (ddd, $J=12.4,7.6$, $1.9 \mathrm{~Hz}, 1 \mathrm{H}), 2.00(\mathrm{t}, J=12.4,11.3 \mathrm{~Hz}, 1 \mathrm{H}), 1.27(\mathrm{~d}, J=6.7 \mathrm{~Hz}, 3 \mathrm{H}) .{ }^{13} \mathrm{C} \mathrm{NMR}(101 \mathrm{MHz}$, $\left.\mathrm{CDCl}_{3}\right) \delta 153.4,152.3,133.7,128.6,124.5,123.1,110.8,107.4,51.4,50.8,47.2,44.7,38.0$, 19.3/13.6. ESI-HRMS calcd for $\left[\mathrm{C}_{14} \mathrm{H}_{14} \mathrm{NaO}_{2}{ }^{+}, \mathrm{M}+\mathrm{Na}\right]^{+}$: 237.0886, Found 237.0890. 


\section{Deuterium Experiments}

\section{Synthesis of 4,4-bis $((E)$-allyl-3- $d)$ isochroman-3-one $\left(1 \mathrm{a}-d^{1}\right)^{5}$}
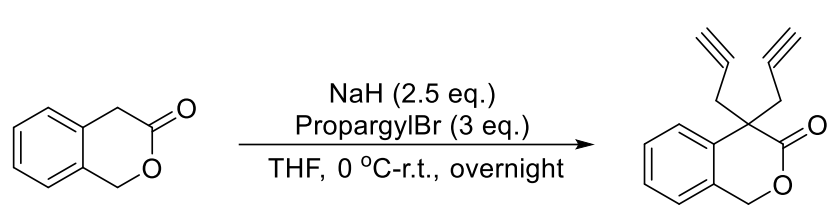
(i) $\mathrm{Cp}_{2} \mathrm{ZrClH}$ (3 eq.) $\mathrm{DCM}, 0^{\circ} \mathrm{C}, 12 \mathrm{~h}$
(ii) $\mathrm{D}_{2} \mathrm{O}$ (3 eq.)
$\mathrm{DCM}, 0^{\circ} \mathrm{C}, 30 \mathrm{~min}$

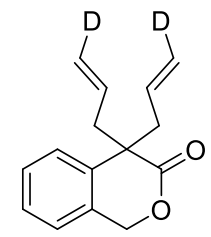

(D > 98\%)

To the sodium hydride $(3.0 \mathrm{~g}, 125 \mathrm{mmol})$ in dry THF $(20 \mathrm{~mL})$, the isochroman-3-one $(11.4$ $\mathrm{g}, 50 \mathrm{mmol})$ dissolved in dry THF $(30 \mathrm{~mL})$ was added dropwise at $0{ }^{\circ} \mathrm{C}$ under the argon. The mixture was allowed to warm to room temperature for $1 \mathrm{~h}$, and then cooled to $0^{\circ} \mathrm{C}$. Propargyl bromide (17.7 g, $150 \mathrm{mmol})$ was added dropwise, and the mixture was allowed to warm to room temperature overnight. After cooling to $0{ }^{\circ} \mathrm{C}$, the mixture was quenched with saturated $\mathrm{NH}_{4} \mathrm{Cl}$, extracted by ethyl acetate $(3 \times 50 \mathrm{~mL})$. The combined organic phase was washed with saturated $\mathrm{NH}_{4} \mathrm{Cl}$, dried over $\mathrm{Na}_{2} \mathrm{SO}_{4}$ and concentrated. The residue was purified by chromatography (ethyl acetate /petroleum ether, 1:2, v/v) to give 4,4-di(prop-2-yn-1yl)isochroman-3-one (3.4 g, 30\% yield) as a yellow solid.

To a Schlenk tube was added 4,4-di(prop-2-yn-1-yl)isochroman-3-one (1.34 g, 6 mmol), $\mathrm{Cp}_{2} \mathrm{ZrClH}(4.6 \mathrm{~g}, 18 \mathrm{mmol})$, and anhydrous dichloromethane $(15 \mathrm{~mL})$ under an argon atmosphere. The mixture was stirred at $0{ }^{\circ} \mathrm{C}$ for $12 \mathrm{~h}$. The mixture was quenched with $\mathrm{D}_{2} \mathrm{O}(3$ $\mathrm{ml}$ ), and the solution was stirred at $0{ }^{\circ} \mathrm{C}$ for $30 \mathrm{~min}$. After removal of the solvent, the residue was purified by column chromatography on silica gel (ethyl acetate/petroleum ether, 1:10, v/v) to afford 4,4-bis $((E)$-allyl-3- $d)$ isochroman-3-one (0.78 g, 57\% yield).

\section{4,4-bis $\left((E)\right.$-allyl-3-d)isochroman-3-one (1a- $\left.d^{1}\right)$}

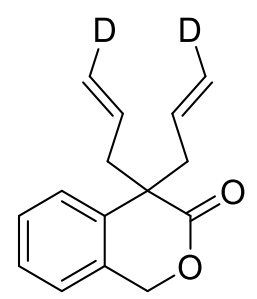

Colorless liquid, TLC $R_{f}=0.58(\mathrm{PE} / \mathrm{EA}=5: 1, \mathrm{v} / \mathrm{v}) .{ }^{1} \mathrm{H}$ NMR $\left(400 \mathrm{MHz}, \mathrm{CDCl}_{3}\right) \delta 7.32(\mathrm{ddtd}$, $J=21.5,15.0,7.6,2.2 \mathrm{~Hz}, 3 \mathrm{H}), 7.12(\mathrm{dd}, J=7.6,2.4 \mathrm{~Hz}, 1 \mathrm{H}), 5.61-5.41(\mathrm{~m}, 2 \mathrm{H}), 5.36(\mathrm{~d}, J$ $=2.5 \mathrm{~Hz}, 2 \mathrm{H}), 5.03(\mathrm{dd}, J=17.0,2.5 \mathrm{~Hz}, 2 \mathrm{H}), 2.89(\mathrm{ddd}, J=14.4,7.2,2.4 \mathrm{~Hz}, 2 \mathrm{H}), 2.64$ (ddd, $J=14.2,7.3,2.4 \mathrm{~Hz}, 2 \mathrm{H}) .{ }^{13} \mathrm{C} \mathrm{NMR}\left(101 \mathrm{MHz}, \mathrm{CDCl}_{3}\right) \delta 173.2,134.6,132.5,132.3,130.6$, $128.2,126.9,126.4,123.9,119.6-118.5$ (m), 69.2, 50.1, 43.1. 


\section{Synthesis of 4,4-bis(allyl-2-d)isochroman-3-one (1a- $\left.d^{2}\right)$}<smiles>C#CCC1(CC#C)C(=O)OCc2ccccc21</smiles>

(i) $\mathrm{Cp}_{2} \mathrm{ZrCID}$ (3 eq.) $\mathrm{DCM}, 0^{\circ} \mathrm{C}, 12 \mathrm{~h}$

(ii) $\mathrm{H}_{2} \mathrm{O}$ (3 eq.) $\mathrm{DCM}, 0^{\circ} \mathrm{C}, 30 \mathrm{~min}$

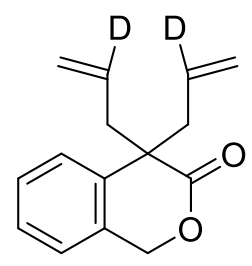

$(\mathrm{D}>98 \%)$

To a Schlenk tube was added 4,4-di(prop-2-yn-1-yl)isochroman-3-one (1.34 g, 6 mmol), $\mathrm{Cp}_{2} \mathrm{ZrClD}$ (4.6 g, $\left.18 \mathrm{mmol}\right)$, and anhydrous dichloromethane $(15 \mathrm{~mL})$ under an argon atmosphere. The mixture was stirred at $0{ }^{\circ} \mathrm{C}$ for $12 \mathrm{~h}$. The mixture was quenched with $\mathrm{H}_{2} \mathrm{O}(3$ $\mathrm{ml}$ ), and the solution was stirred at $0{ }^{\circ} \mathrm{C}$ for $30 \mathrm{~min}$. After removal of the solvent, the residue was purified by column chromatography on silica gel (ethyl acetate/petroleum ether, 1:10, v/v) to afford 4,4-bis(allyl-2-d)isochroman-3-one (0.55 g, $40 \%$ yield).

\section{4,4-bis(allyl-2-d)isochroman-3-one (1a- $\left.d^{2}\right)$}

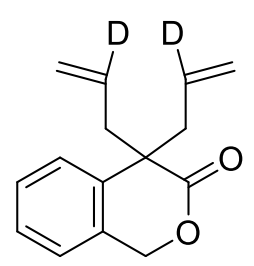

Colorless liquid, TLC $R_{f}=0.58(\mathrm{PE} / \mathrm{EA}=5: 1, \mathrm{v} / \mathrm{v}) .{ }^{1} \mathrm{H}$ NMR $\left(400 \mathrm{MHz}, \mathrm{CDCl}_{3}\right) \delta 7.48-$ $7.22(\mathrm{~m}, 4 \mathrm{H}), 7.11$ (d, $J=7.6 \mathrm{~Hz}, 1 \mathrm{H}), 5.37$ (s, 2H), 5.02 (d, $J=18.8 \mathrm{~Hz}, 4 \mathrm{H}), 2.89$ (d, $J=13.9$ $\mathrm{Hz}, 2 \mathrm{H}), 2.63(\mathrm{~d}, J=13.9 \mathrm{~Hz}, 2 \mathrm{H}) .{ }^{13} \mathrm{C} \mathrm{NMR}\left(101 \mathrm{MHz}, \mathrm{CDCl}_{3}\right) \delta 173.4,134.9,132.3(\mathrm{t}, J=$ $23.7 \mathrm{~Hz}), 130.8,128.4,127.1,126.6,124.1,119.4,69.5,50.3,43.3$.

\section{Cyclizations of deuterated isochroman-3-ones}

To the first Schlenk tube was added $\left[\mathrm{Ni}(\text { allyl)Br }]_{2}\right.$, ligand, and anhydrous dichloromethane $(1.5 \mathrm{~mL})$ under an argon atmosphere. The mixture was stirred at room temperature for $15 \mathrm{~min}$. To the second Schlenk tube was added $\mathrm{NaBAr}_{\mathrm{F}}$ and 1,6-diene $(0.1 \mathrm{mmol})$. The solution in the first Schlenk tube was transferred into the second Schlenk tube. The resulting mixture was stirred at $0{ }^{\circ} \mathrm{C}$ for $48 \mathrm{~h}$. After removal of the solvent, the residue was purified by column chromatography on silica gel (ethyl acetate/petroleum ether, 1:10, v/v) to afford product. 
(a)

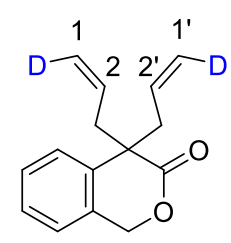

$$
\begin{gathered}
1 \mathbf{a}-d^{1} \\
D>98 \%
\end{gathered}
$$

(b)<smiles>[2H]C(=[IH])CC1(CC([2H])([2H])F)C(=O)OCc2ccccc21</smiles>

$$
\begin{gathered}
1 \mathrm{a}-d^{2} \\
D>98 \%
\end{gathered}
$$

[Ni(allyl)Br $]_{2}(2.5 \mathrm{~mol} \%)$

$\mathrm{L}_{7}(5 \mathrm{~mol} \%), \mathrm{NaBAr}_{\mathrm{F}}(6 \mathrm{~mol} \%)$

$\mathrm{CH}_{2} \mathrm{Cl}_{2}, 0{ }^{\circ} \mathrm{C}, 48 \mathrm{~h}$

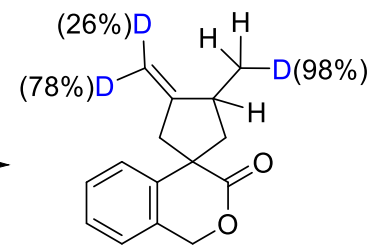

2a- $d^{1}$

$80 \%$ yield<smiles>C/C=C1\CC2(C(=O)OCc3ccccc32)C(O[13CH3])C1[18OH]</smiles>

$2 a-d^{2}$

$79 \%$ yield

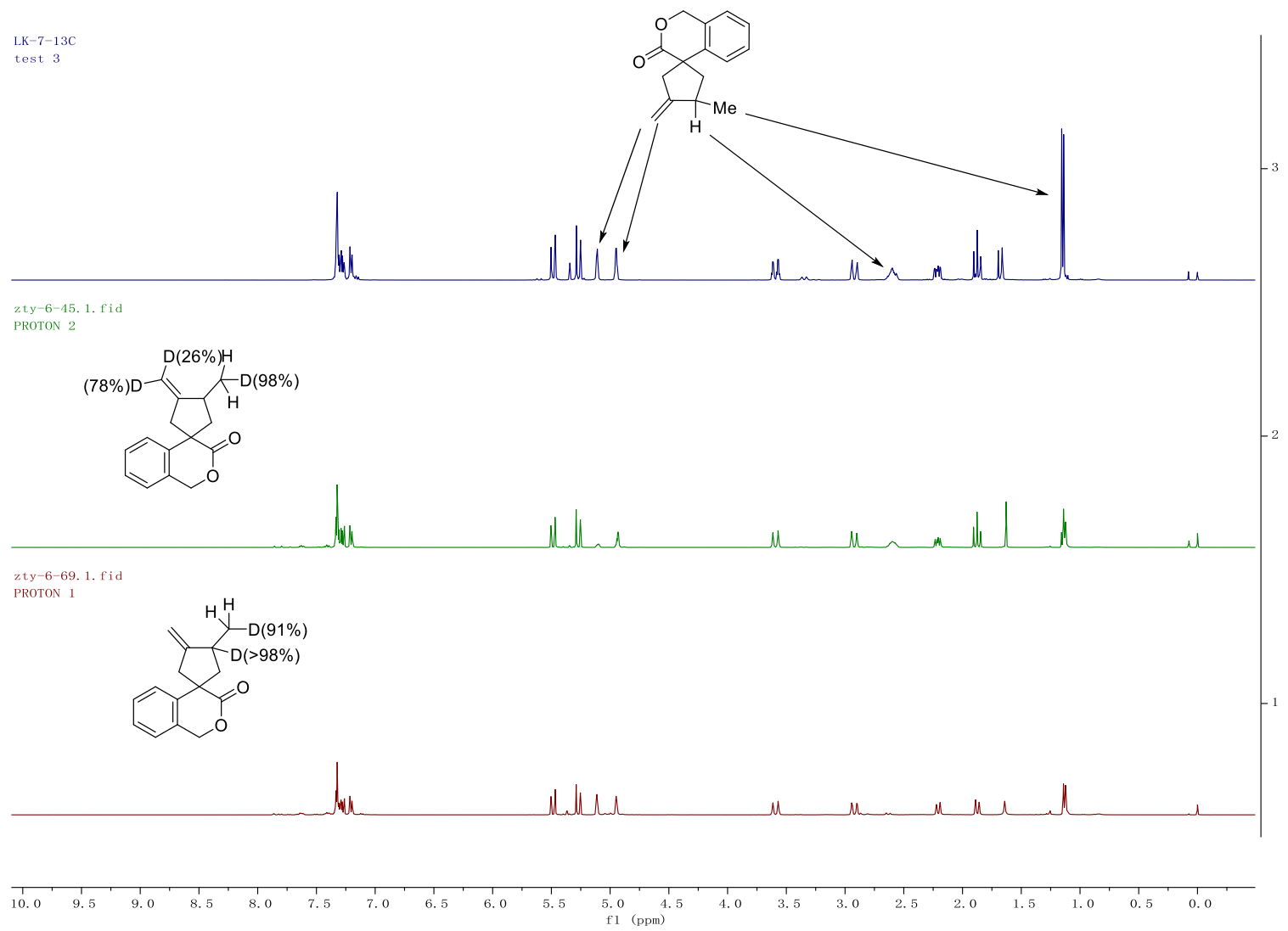


Determined the absolute configuration of $2 \mathrm{~s}$

Synthesis of (1-(3-fluoro-2-(hydroxymethyl)phenyl)-3-methyl-4-methylenecyclopentyl)methanol ( $\left.2 s^{\prime}\right)$

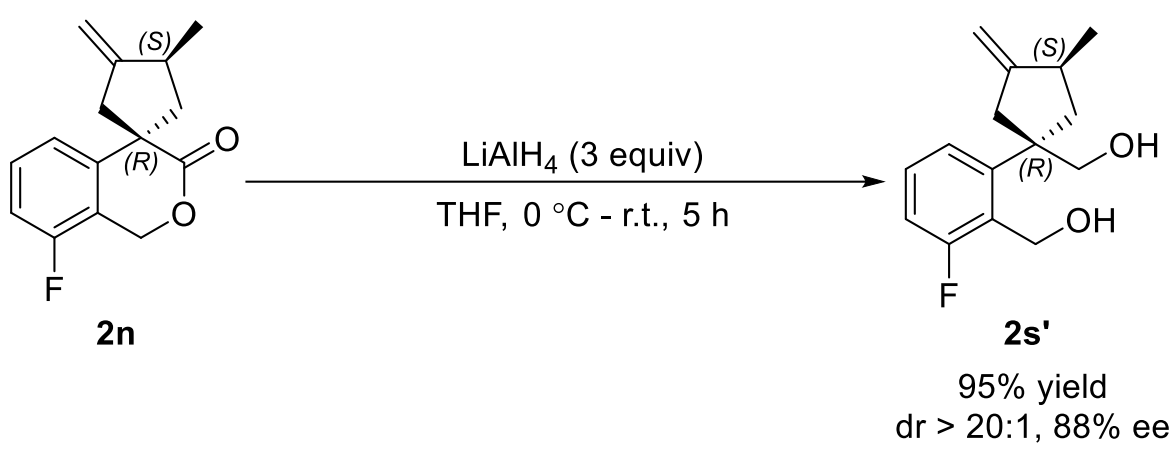

To a Schlenk tube was added $2 \mathrm{n}(19.7 \mathrm{mg}, 0.08 \mathrm{mmol})$ under argon atmosphere. Dry THF $(1.5 \mathrm{~mL})$ was added, and the solution was cooled to $0{ }^{\circ} \mathrm{C}$. To this solution was added $\mathrm{LiAlH}_{4}$ ( $0.096 \mathrm{~mL}, 2.5 \mathrm{M}$ in THF, $0.24 \mathrm{mmol})$ using a slow addition pump. The solution was allowed to warm to room temperature for $5 \mathrm{~h}$. After quenching with saturated $\mathrm{NH}_{4} \mathrm{Cl}$ under the $0{ }^{\circ} \mathrm{C}$, the reaction mixture was extracted with ethyl acetate $(3 \times 5 \mathrm{~mL})$. The extract was concentrated, and the residue was purified by column chromatography (ethyl acetate/petroleum ether, 1:2, $\mathrm{v} / \mathrm{v})$ to give pure (1-(3-fluoro-2-(hydroxymethyl)phenyl)-3-methyl-4-methylenecyclopentyl)methanol (2s') (19.0 mg, 95\% yield). 


\section{X-Ray Diffraction Analysis of Product 2n}

Crystal of $\mathbf{2 n}$ was prepared by evaporation crystallization from $n$-hexane and dichloromethane.

Thermal probability ellipsoids shown at the $50 \%$ probability level.

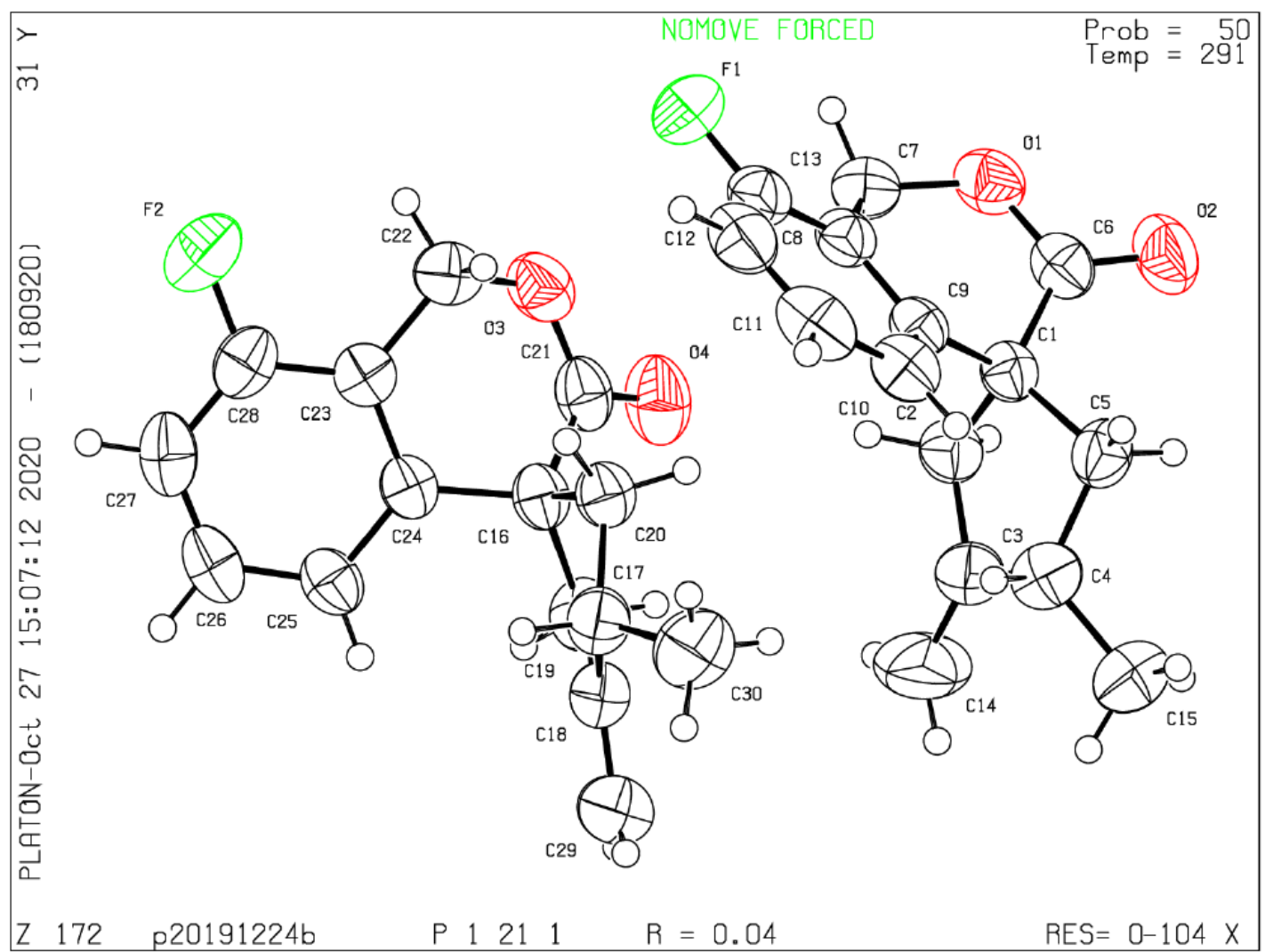


Table S2. Crystal data and structure refinement for compound $\mathbf{2 n}$.

Identification code

Empirical formula

Formula weight

Temperature

Crystal system

Space group

Unit cell dimensions

\section{CCDC 2041656}

C15H15FO2

246.27

$291.15 \mathrm{~K}$

monoclinic

$\mathrm{P} 2_{1}$

$$
\begin{array}{ll}
\mathrm{a}=9.1299(1)) \AA & \alpha=90^{\circ} \\
\mathrm{b}=14.6130(1) \AA & \beta=115.687(6)^{\circ} \\
\mathrm{c}=9.6532(1) \AA & \gamma=90^{\circ} \\
1241.78(2) \AA^{3} &
\end{array}
$$

Volume

$\mathrm{Z}$

Density (calculated)

Absorption coefficient

$\mathrm{F}(000)$

Crystal size

Radiation
4

$1.317 \mathrm{~g} / \mathrm{cm}^{3}$

$0.794 \mathrm{~mm}^{-1}$

520.0

$0.28 \times 0.24 \times 0.22 \mathrm{~mm}^{3}$

$\mathrm{CuK} \alpha$

$(\lambda=1.54184)$ Theta range for data

collection

9.502

to $158.778^{\circ}$

Index ranges

$-11 \leq \mathrm{h} \leq 11,-18 \leq \mathrm{k} \leq 18,-12 \leq 1 \leq 11$

Reflections collected 14985

Independent reflections $5012[$ Rint $=0.0205$,

Rsigma $=0.0165]$ Data/restraints/parameters $5012 / 1 / 328$

Goodness-of-fit on F2

1.100

Final $\mathrm{R}$ indexes $[\mathrm{I}>=2 \sigma(\mathrm{I})] \quad \mathrm{R}_{1}=0.0376, \mathrm{wR}_{2}=0.1107$

Final R indexes

[all data] $\mathrm{R}_{1}=0.0386, \mathrm{wR}_{2}=0.1116$

Largest diff. peak/hole

$0.18 /-0.14$ e. $\AA^{-3}$

Flack parameter

$0.05(10)$ 


\section{NMR Spectra of New Compounds}

(R)-4-(1,10-di([1,1':3',1''-terphenyl]-5'-yl)-4,5,6,7-tetrahydrodiindeno[7,1-de:1',7' fg] $[1,3,2]$ dioxaphosphocin-12-yl)morpholine ((R)-L7)
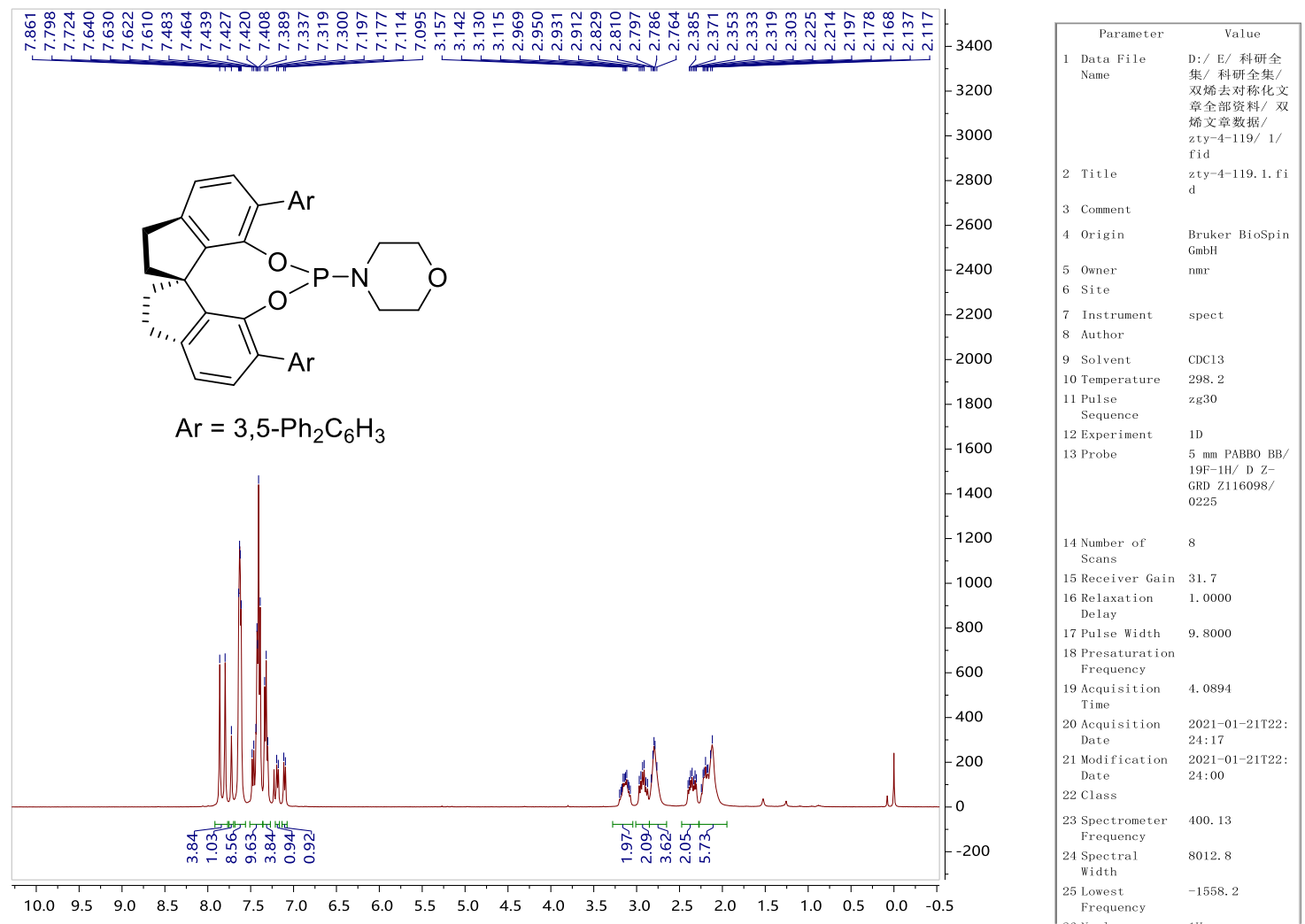

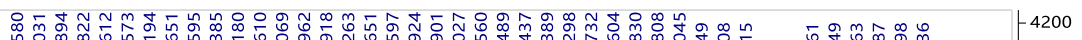

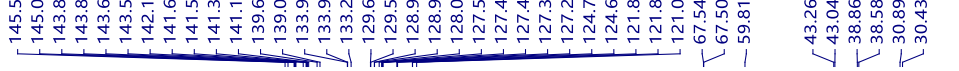<smiles>Brc1ccc2c(c1OP(Oc1c(Br)ccc3c1[C@H]1C[C@@H]3C1)N1CCOCC1)C2</smiles>

$\mathrm{Ar}=3,5-\mathrm{Ph}_{2} \mathrm{C}_{6} \mathrm{H}_{3}$

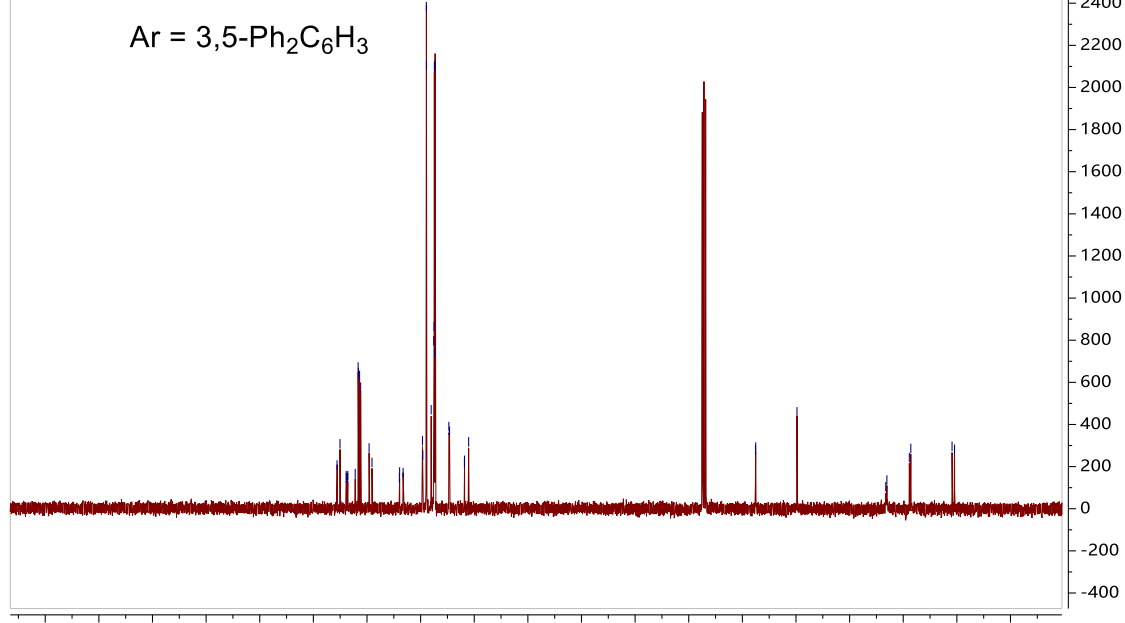

$\begin{array}{lllllllllllllllllll}200 & 190 & 180 & 170 & 160 & 150 & 140 & 130 & 120 & 110 & 100 & 90 & 80 & 70 & 60 & 50 & 40 & 30 & 20\end{array}$

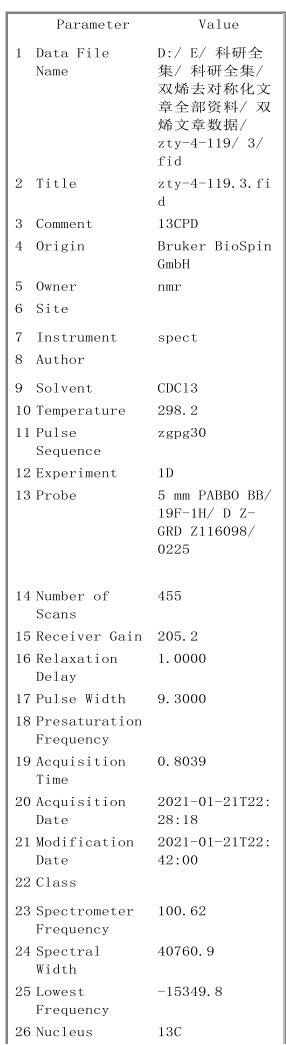


(R)-4-(1,10-bis(3,5-di(naphthalen-2-yl)phenyl)-4,5,6,7-tetrahydrodiindeno[7,1-de:1',7'fg] $[1,3,2]$ dioxaphosphocin-12-yl)morpholine ((R)-L8)

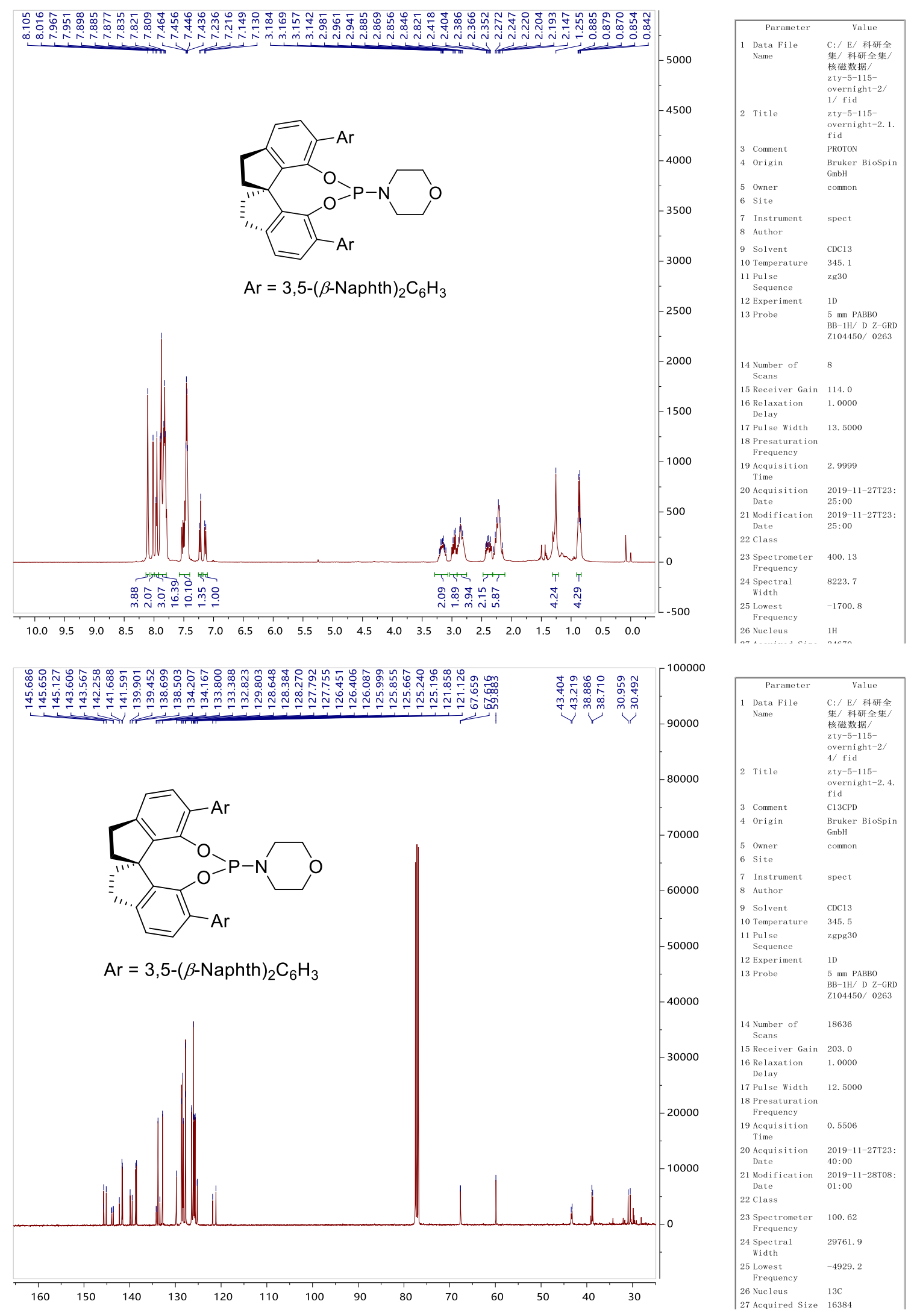


4,4-diallylisochroman-3-one (1a)
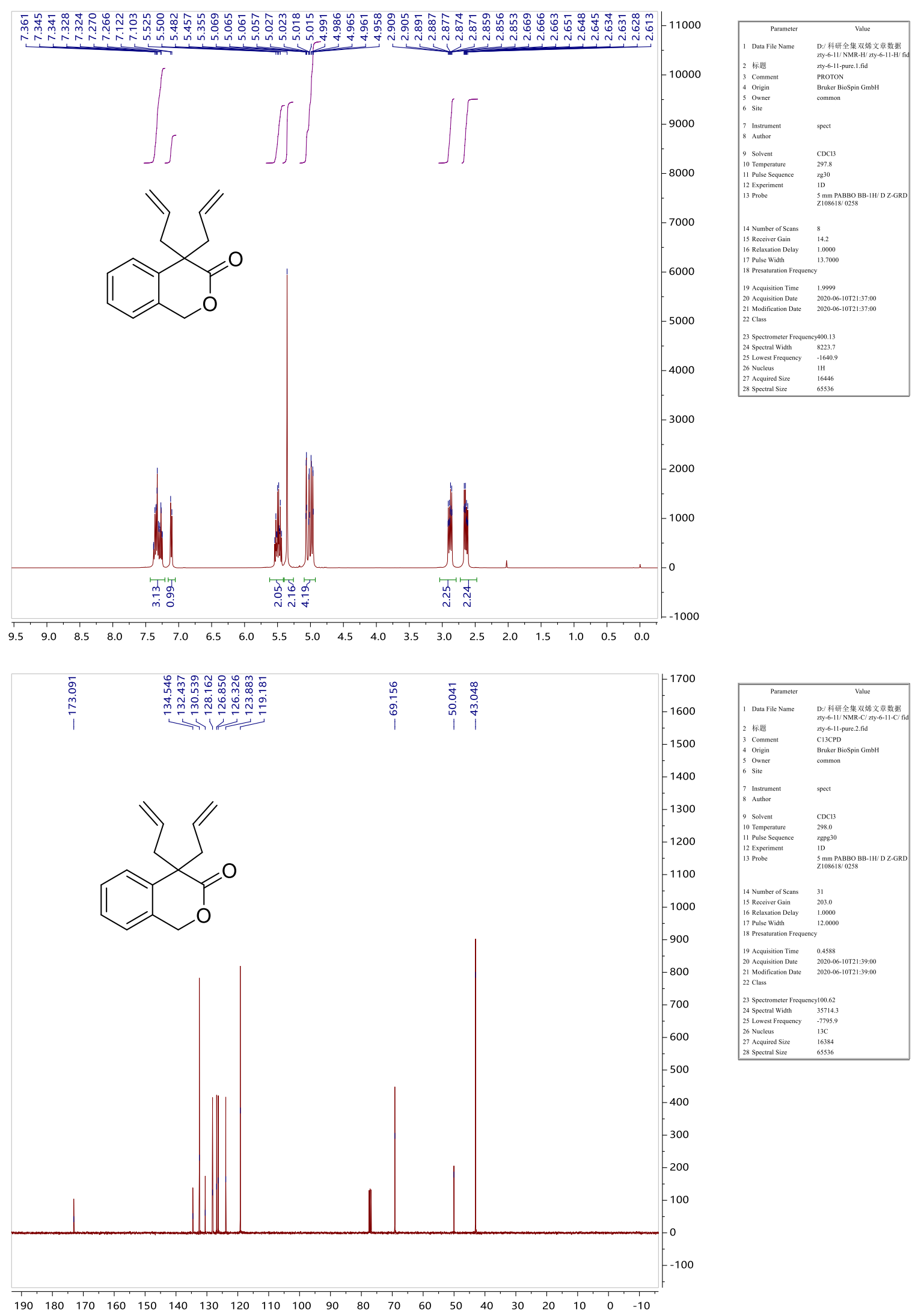
4,4-diallyl-7-methylisochroman-3-one (1b)
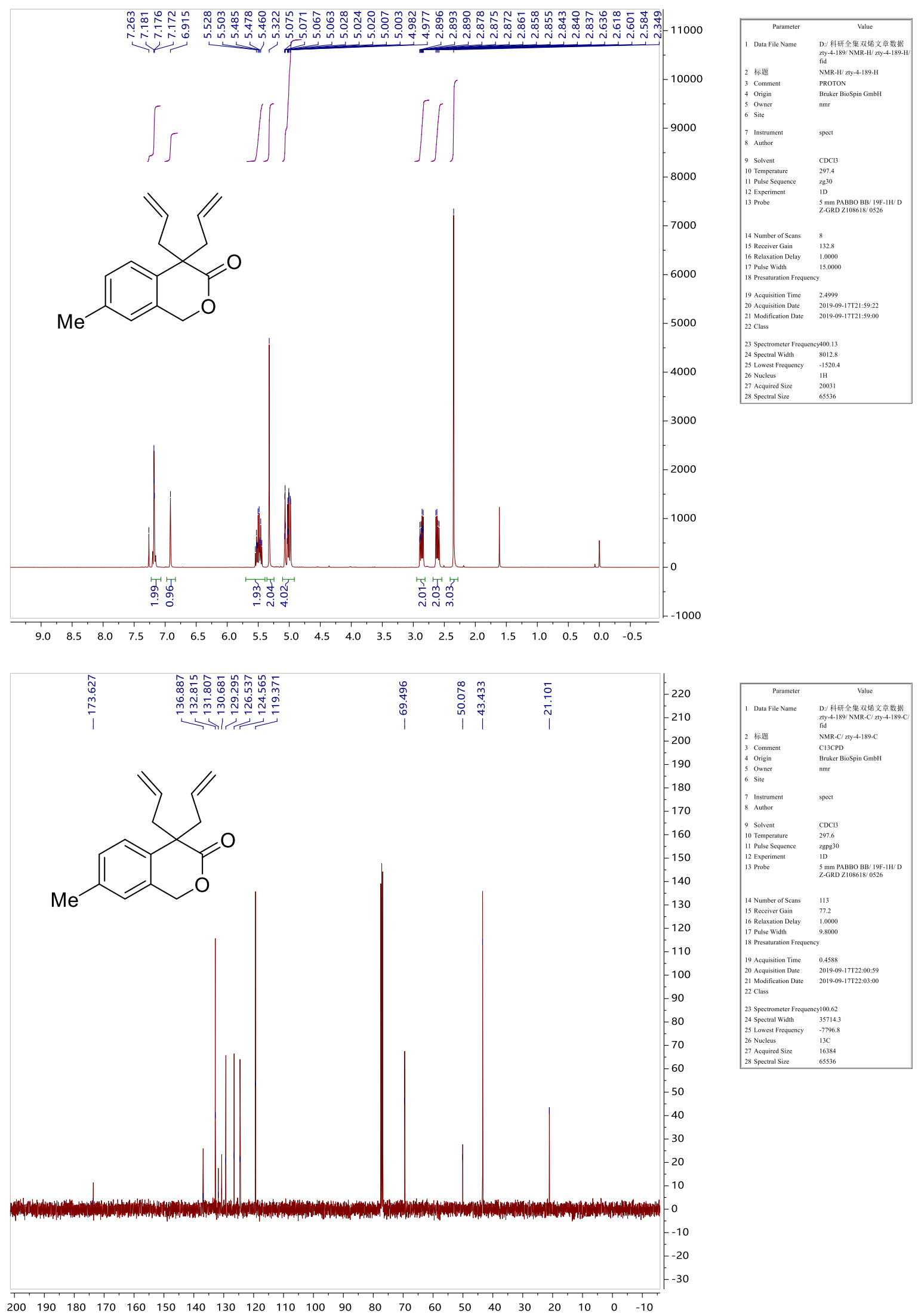
4,4-diallyl-7-chloroisochroman-3-one (1c)
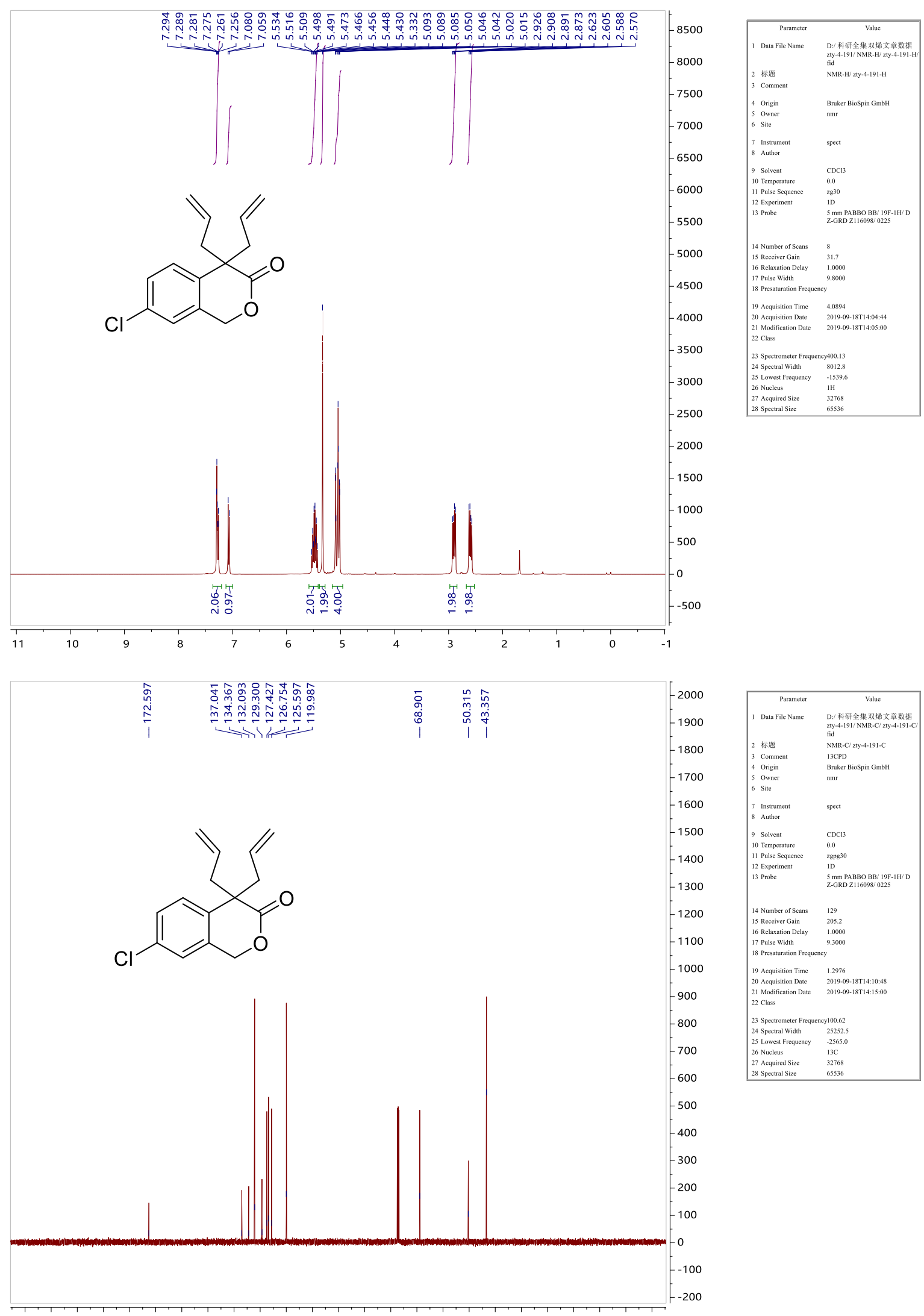

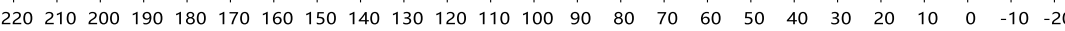


4,4-diallyl-7-methoxyisochroman-3-one (1d)
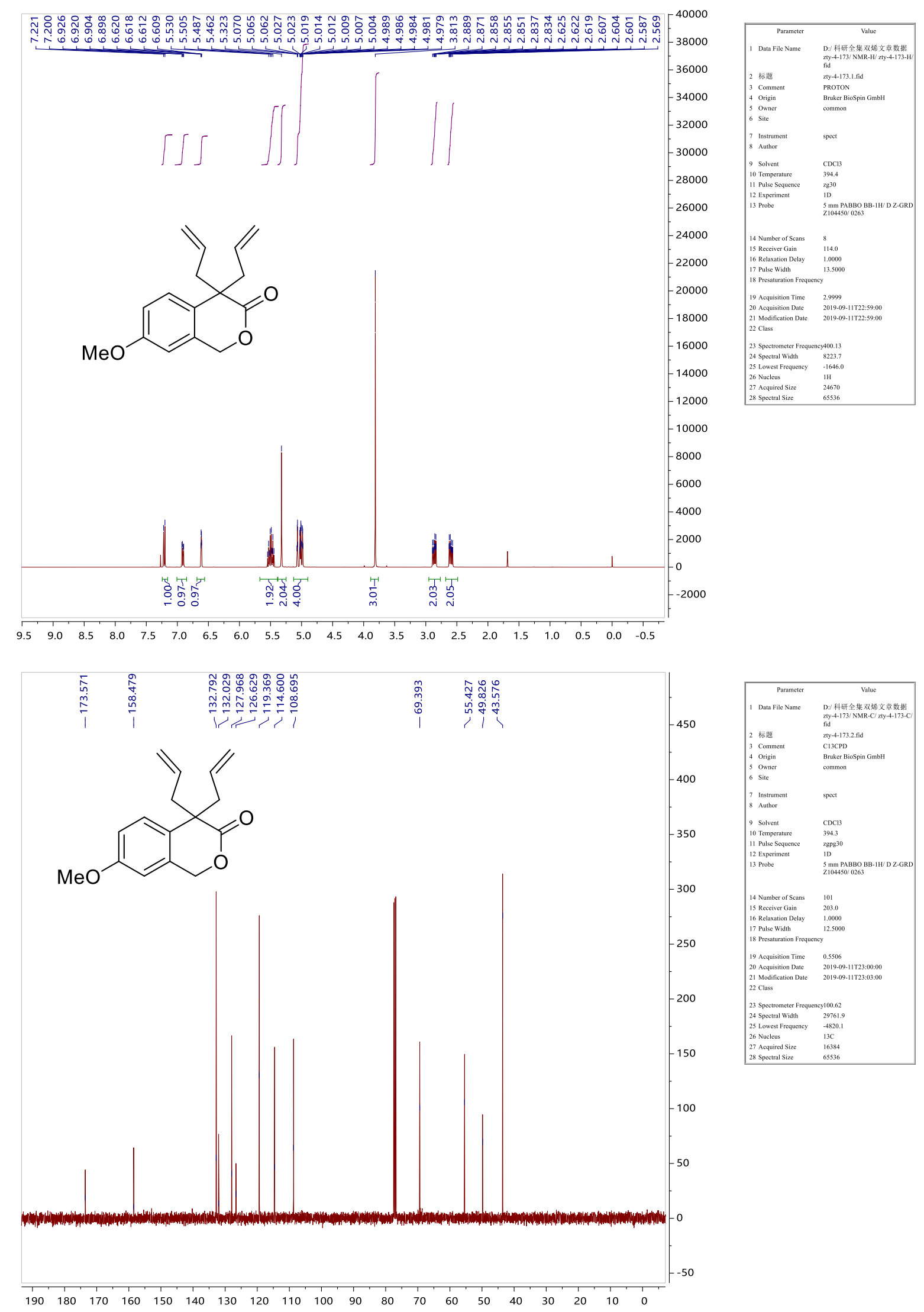
4,4-diallyl-6-methylisochroman-3-one (1e)
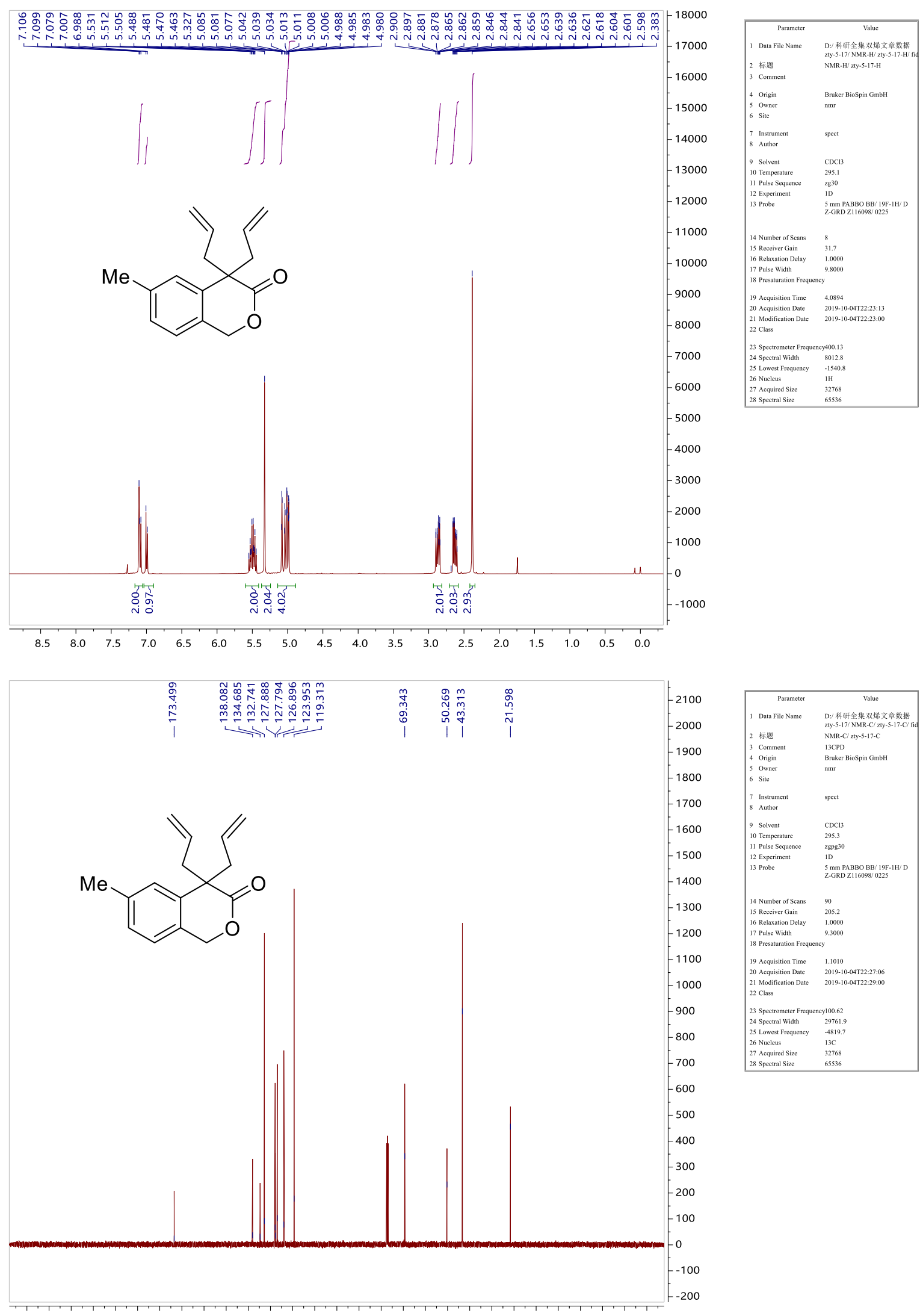

$24023022021020019018017016015014013012011010090 \quad 80 \quad 706050 \quad 40 \quad 30 \quad 20 \quad 10 \quad 0 \quad-10-20-30-40$ 
4,4-diallyl-6-chloroisochroman-3-one (1f)
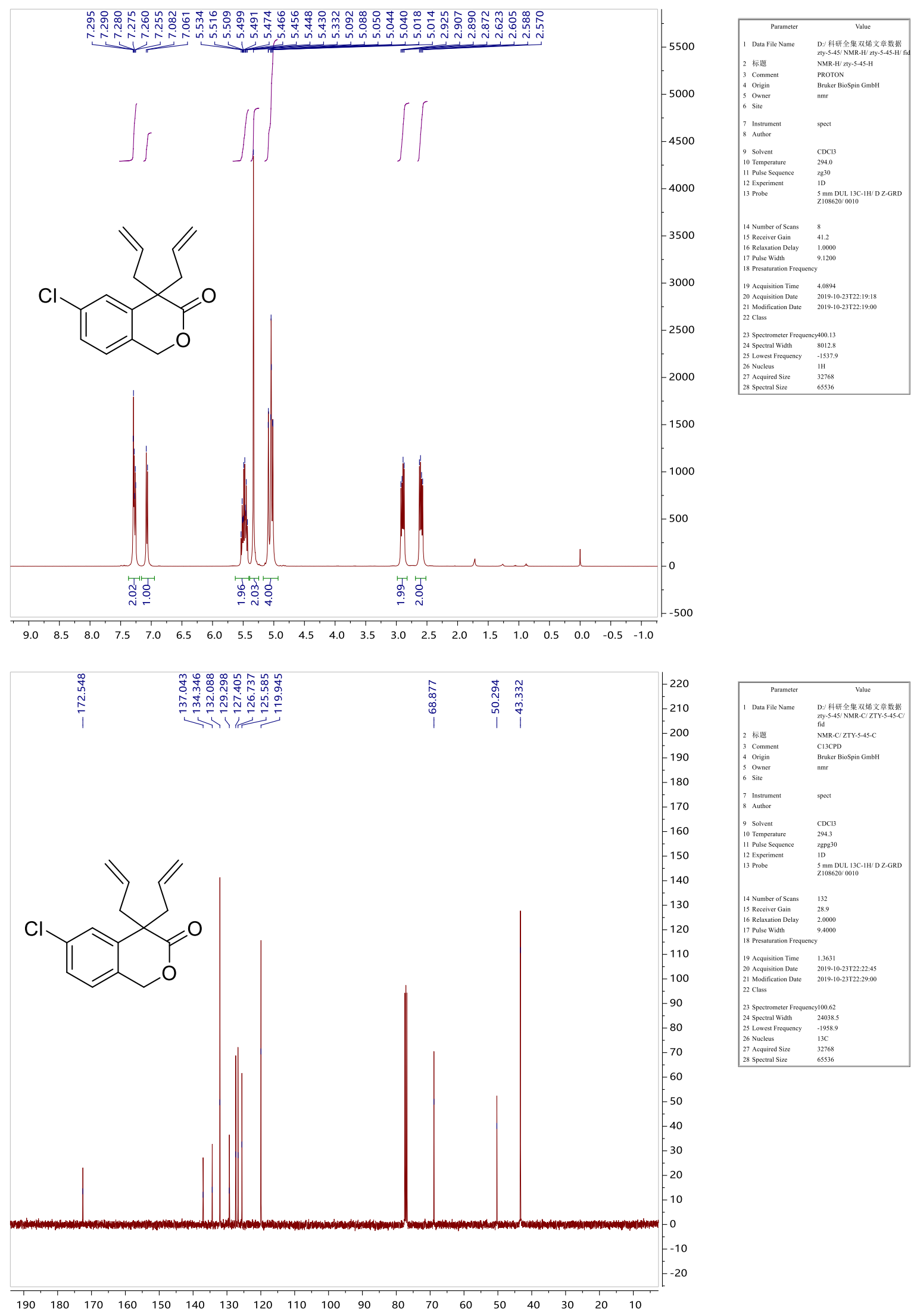


\section{4,4-diallyl-6-methoxyisochroman-3-one (1g)}
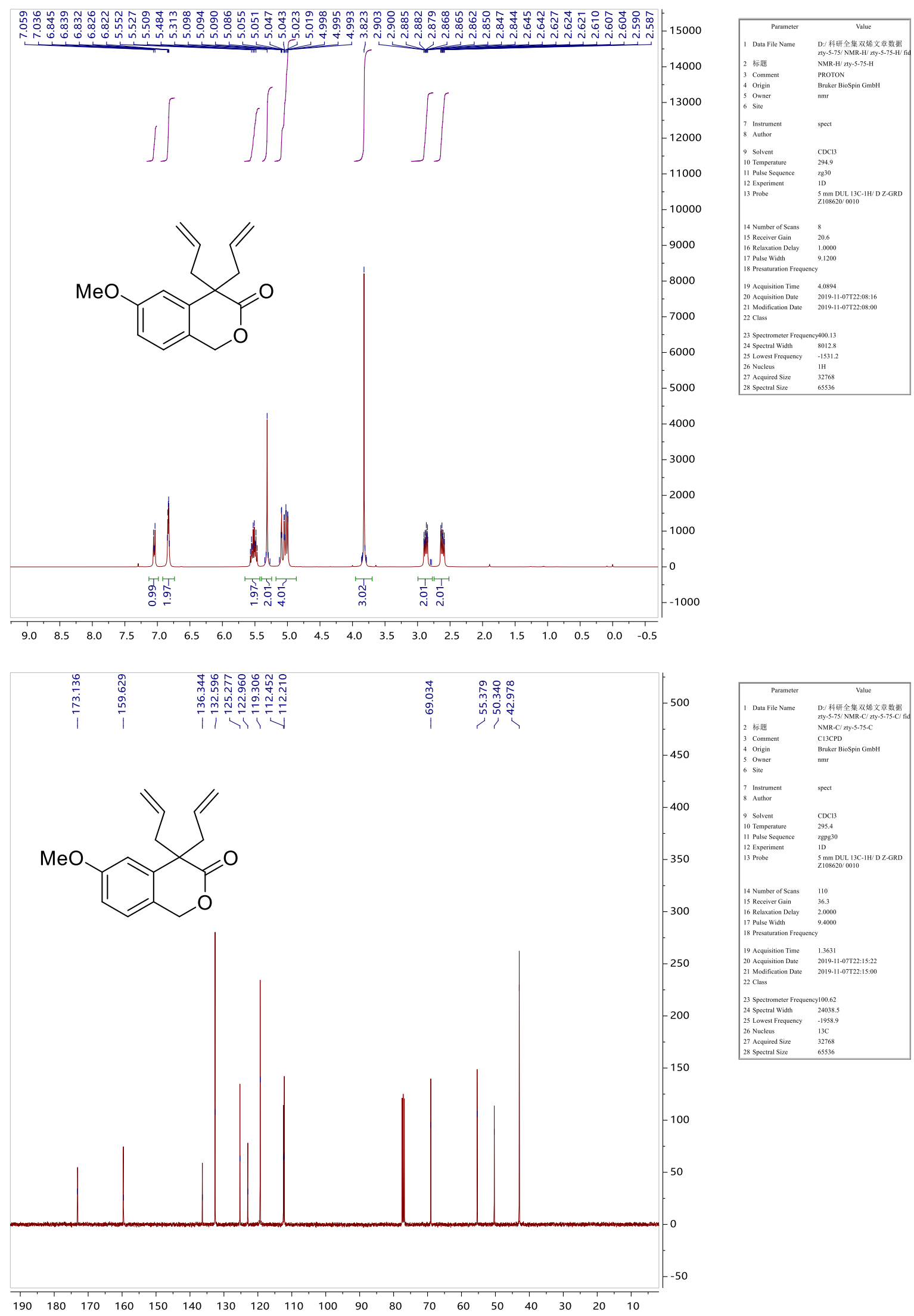


\section{4,4-diallyl-6-bromoisochroman-3-one (1h)}
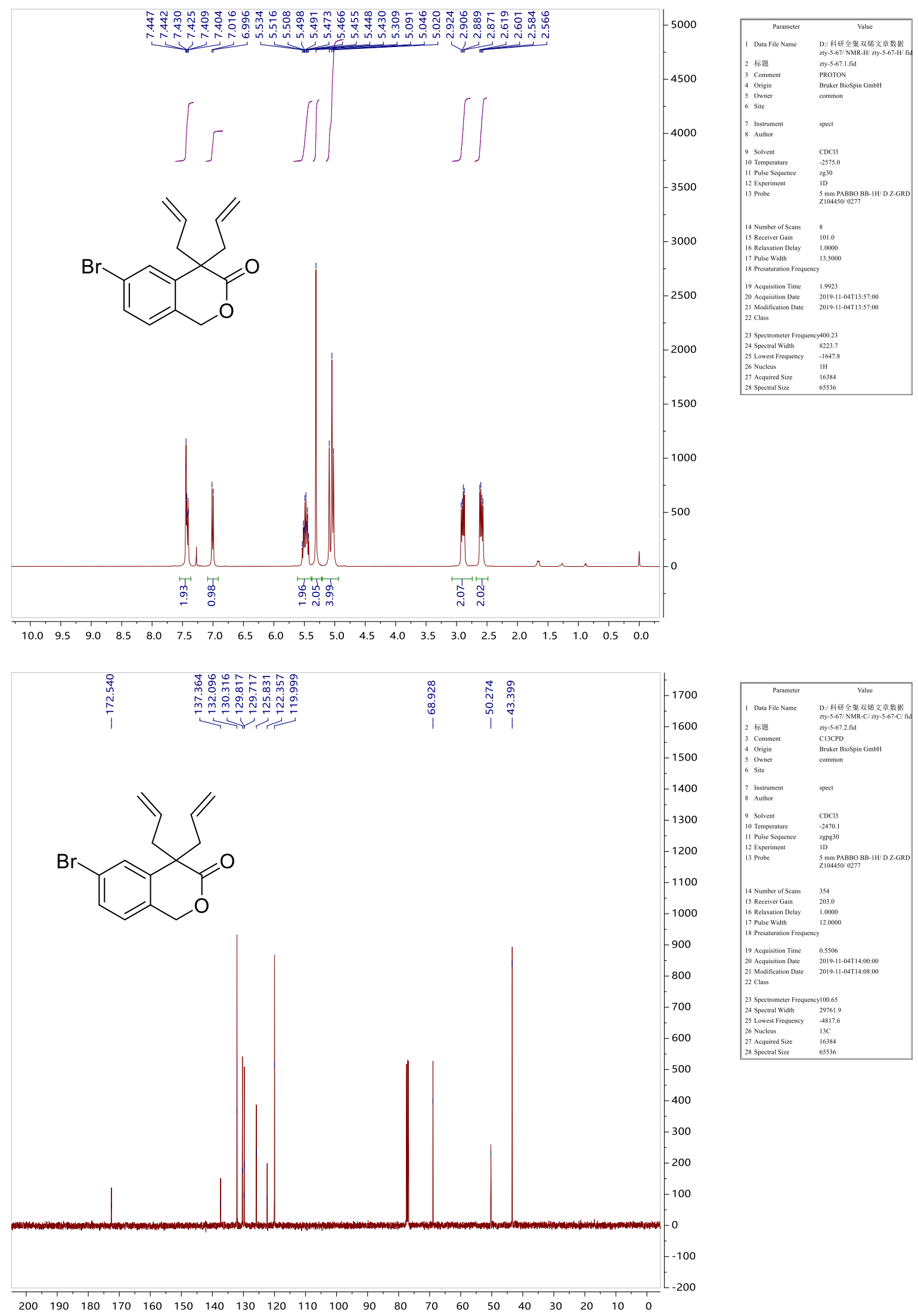

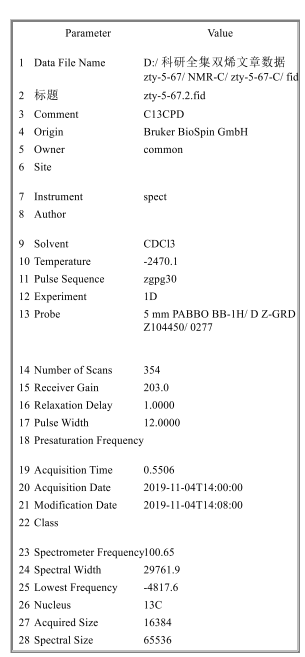



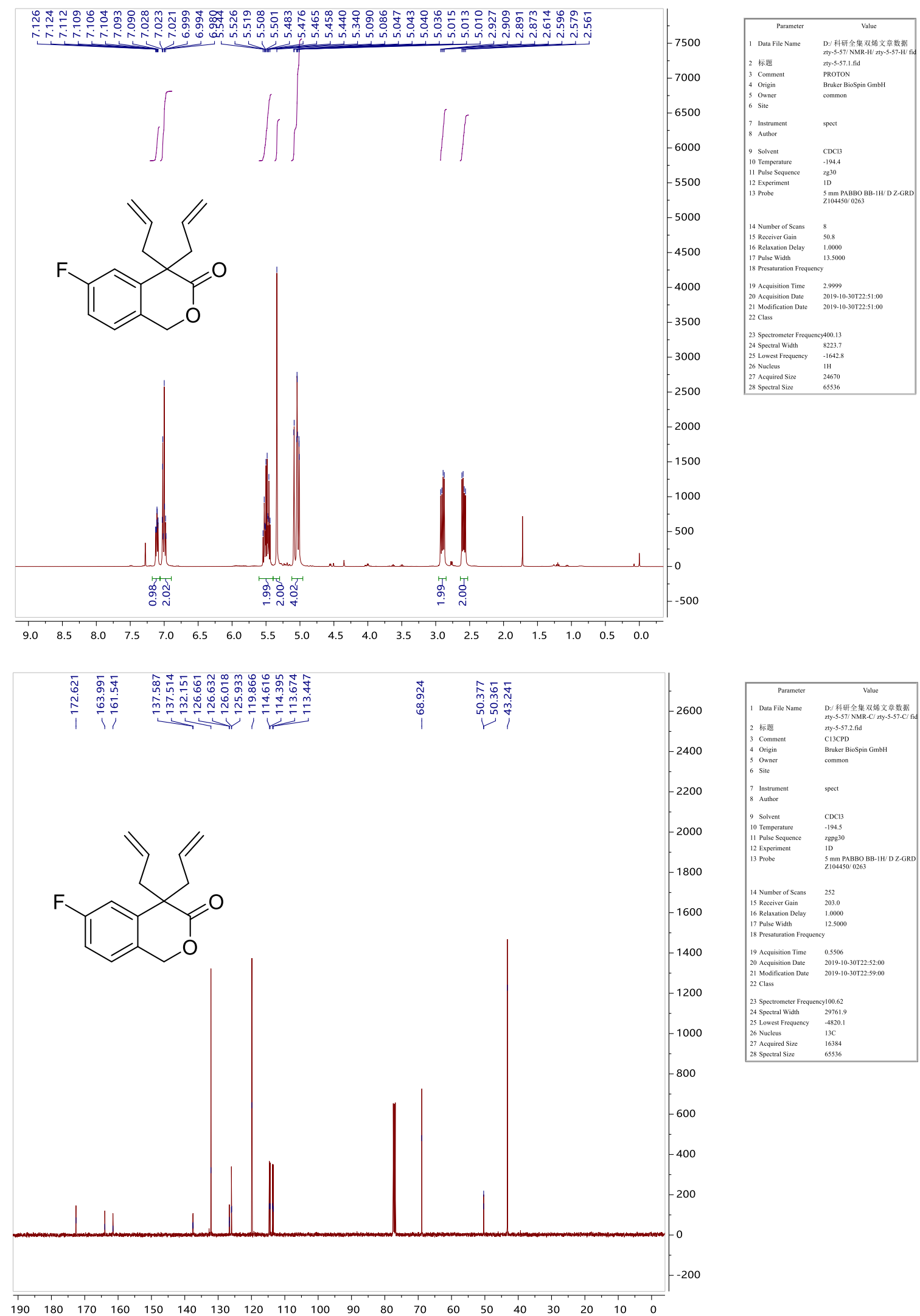


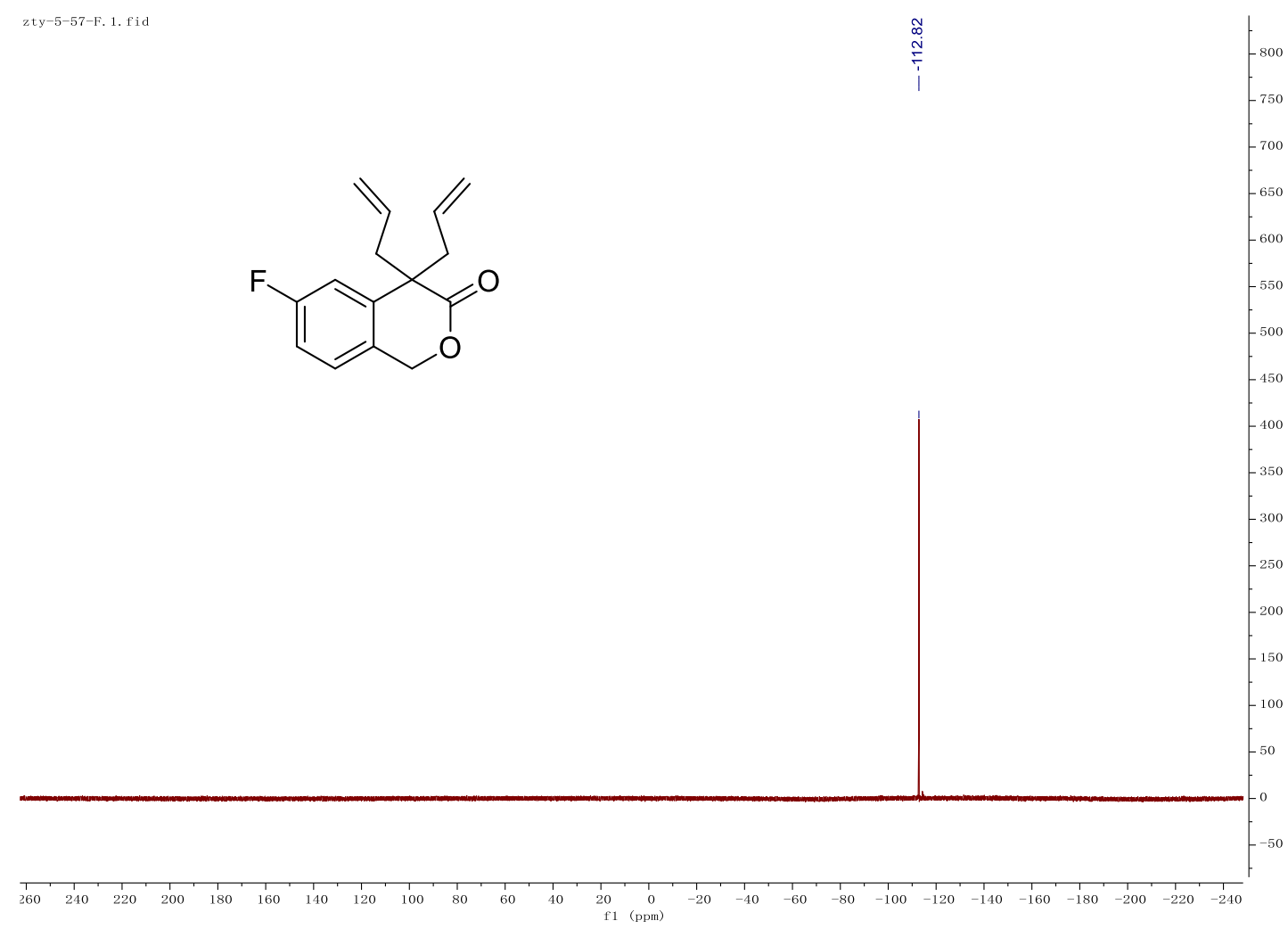


4,4-diallyl-6-(trifluoromethyl)isochroman-3-one (1j)
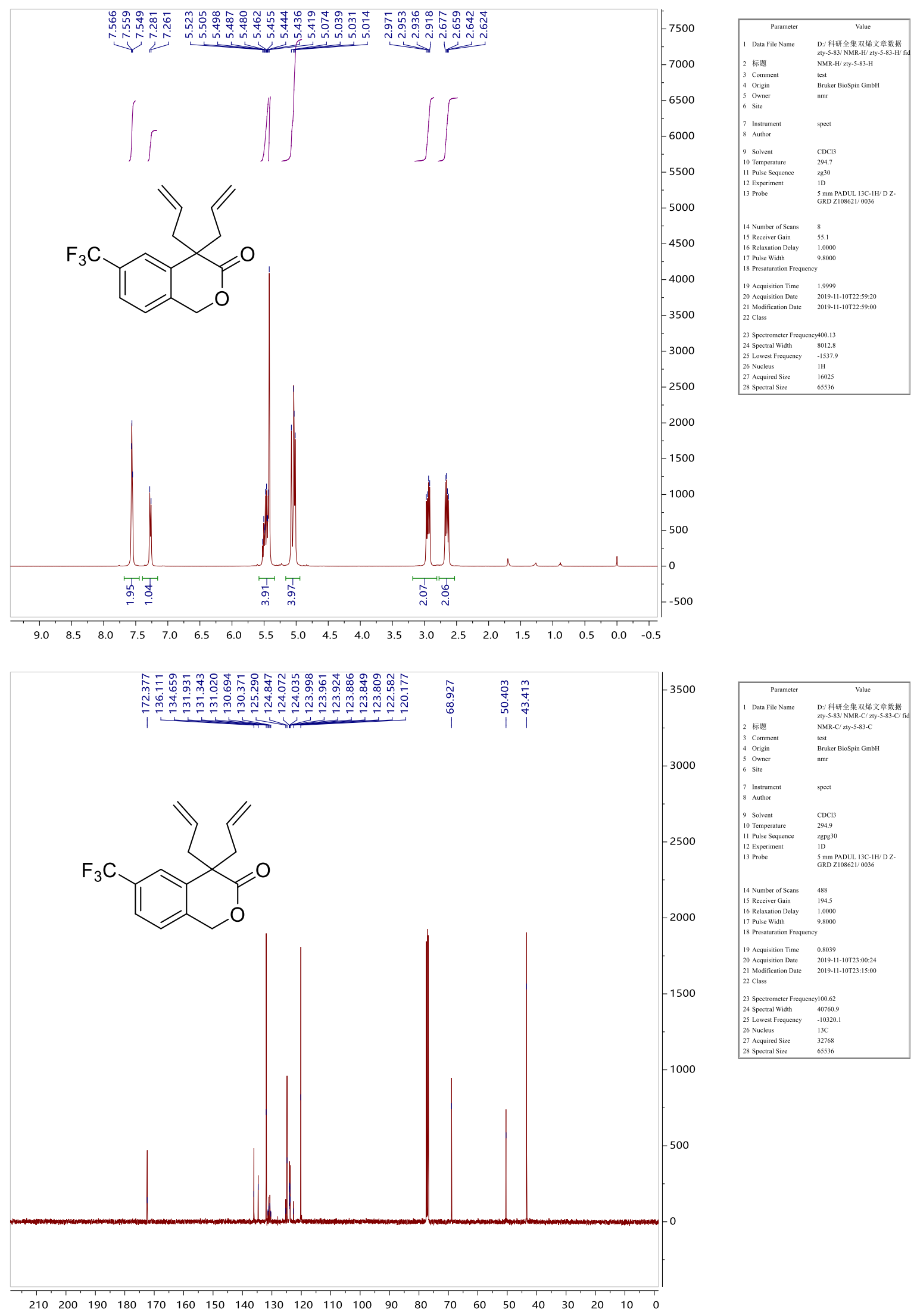


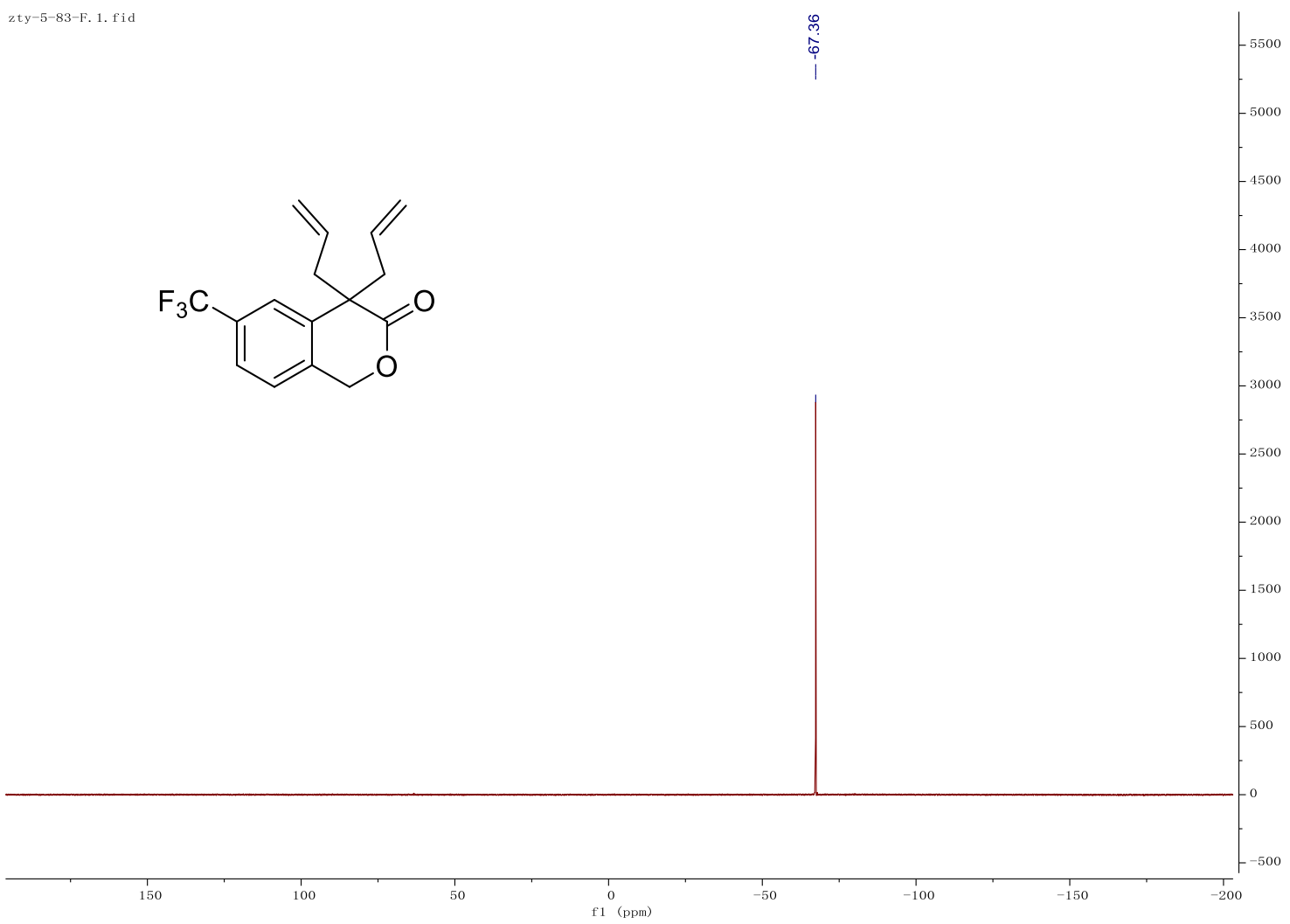


4,4-diallyl-6-(4-nitrophenyl)isochroman-3-one (1k)
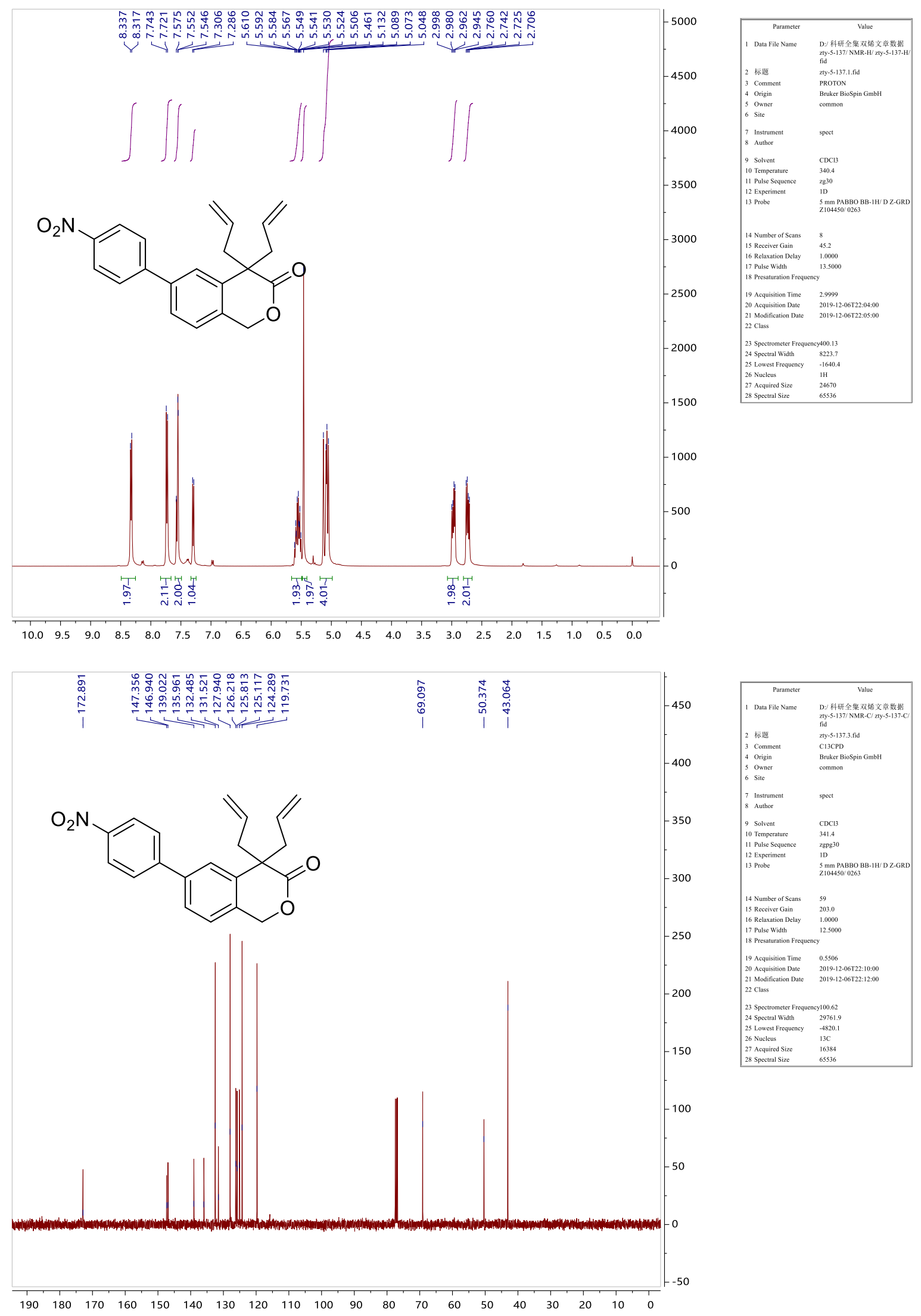
methyl 4-(4,4-diallyl-3-oxoisochroman-6-yl)benzoate (11)

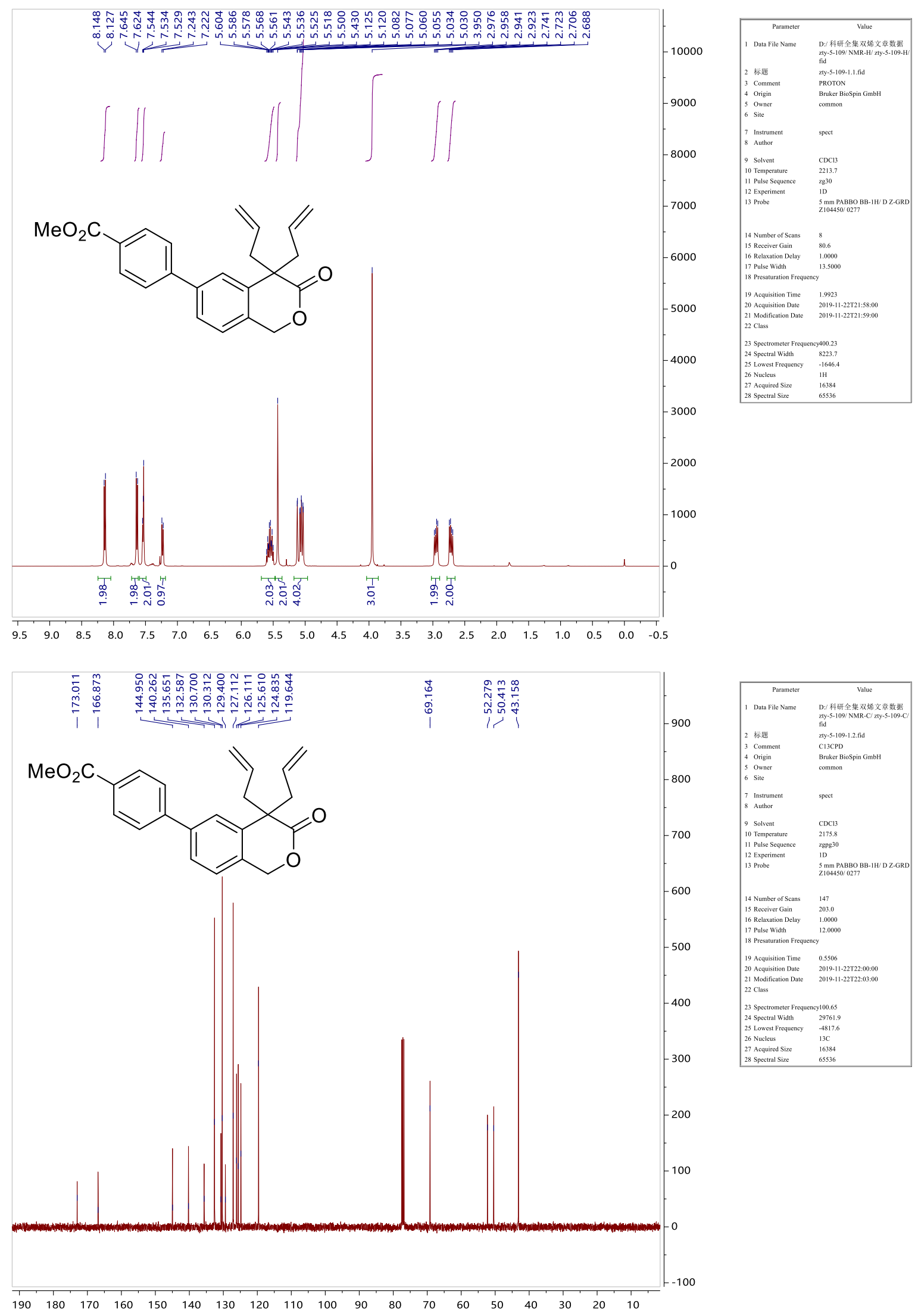




\section{4,4-diallyl-8-methylisochroman-3-one (1m)}
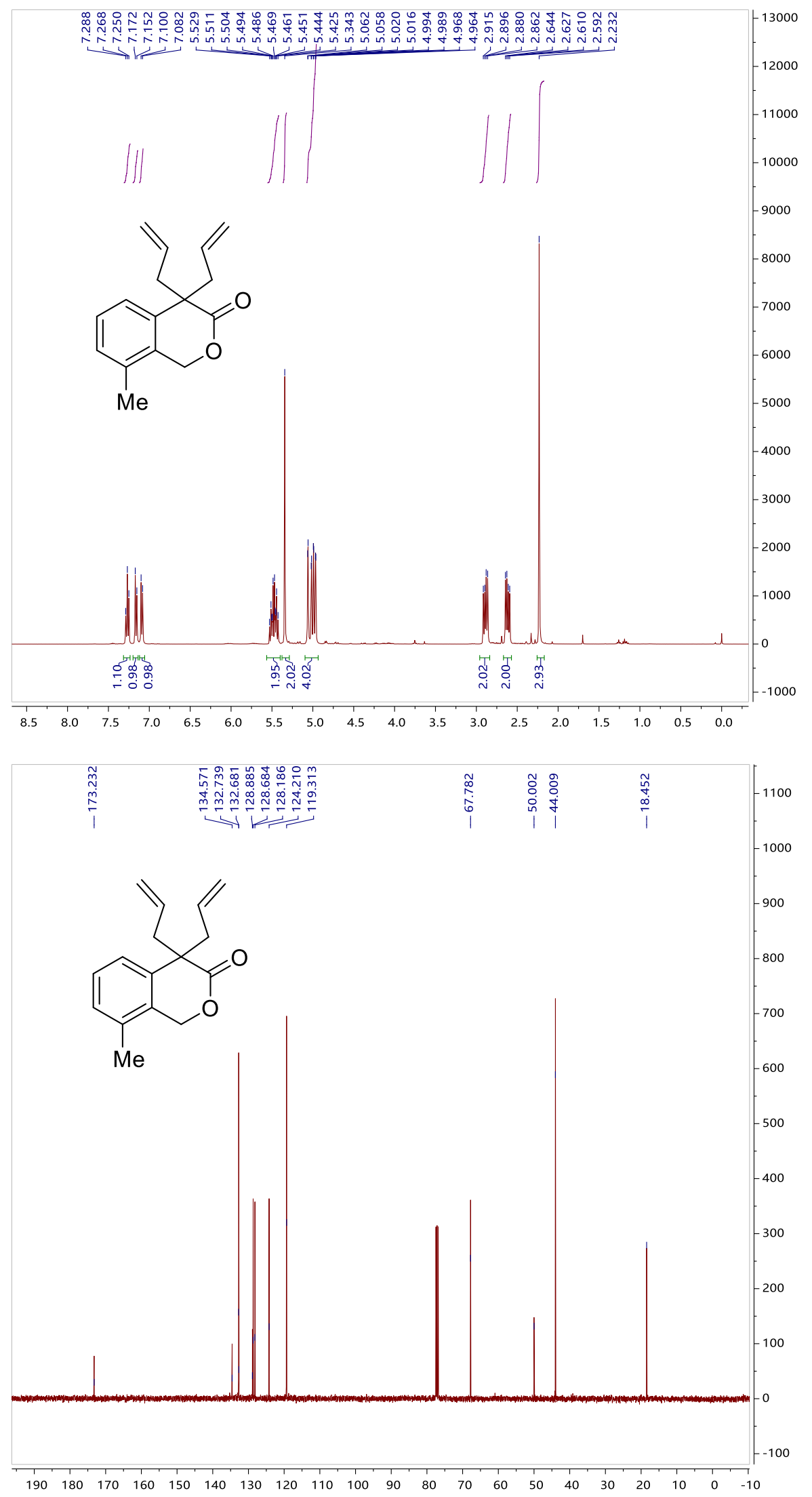
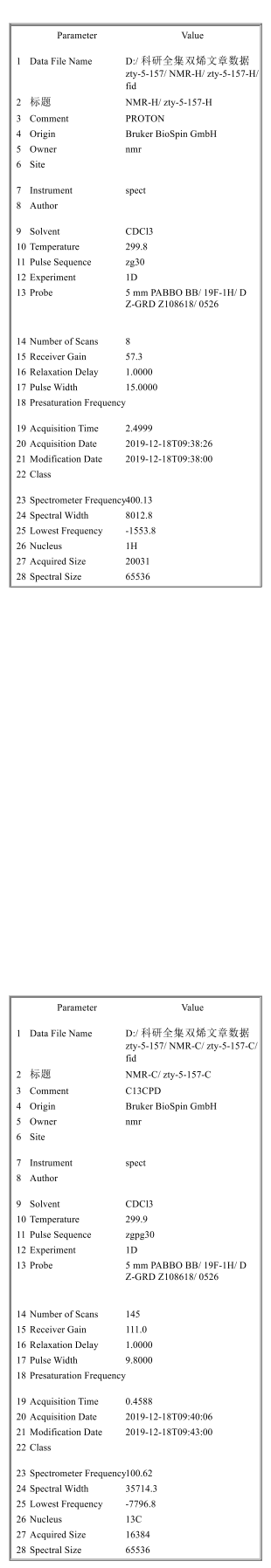
4,4-diallyl-8-fluoroisochroman-3-one (1n)
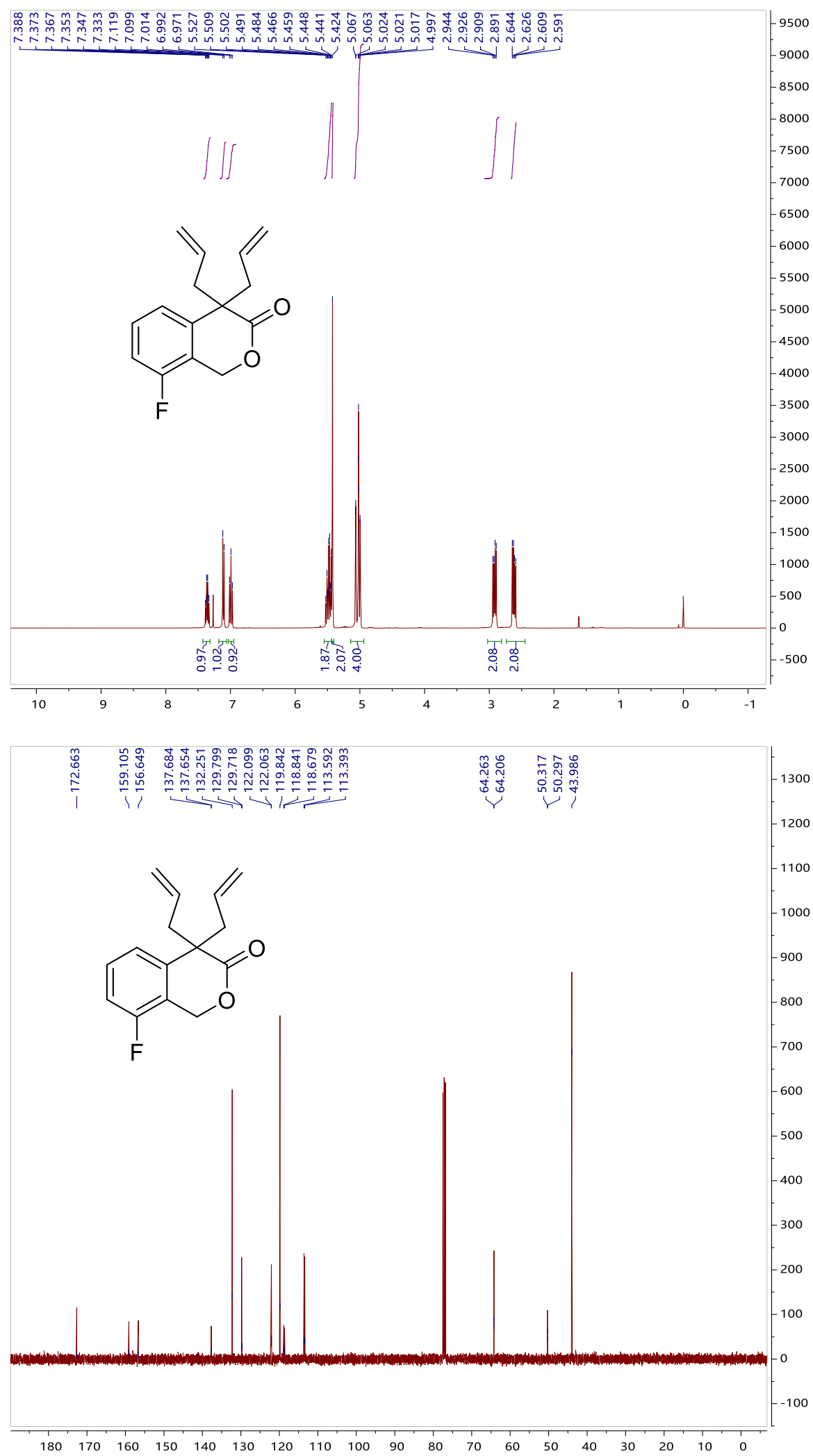
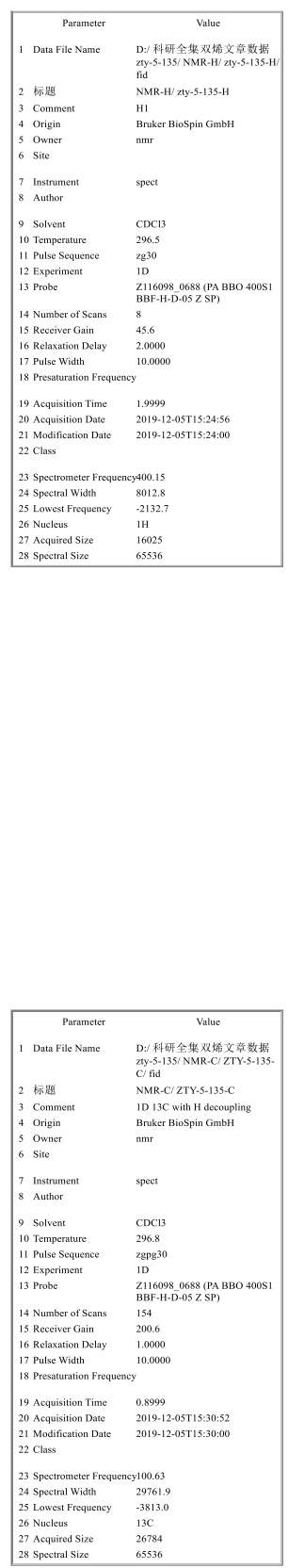


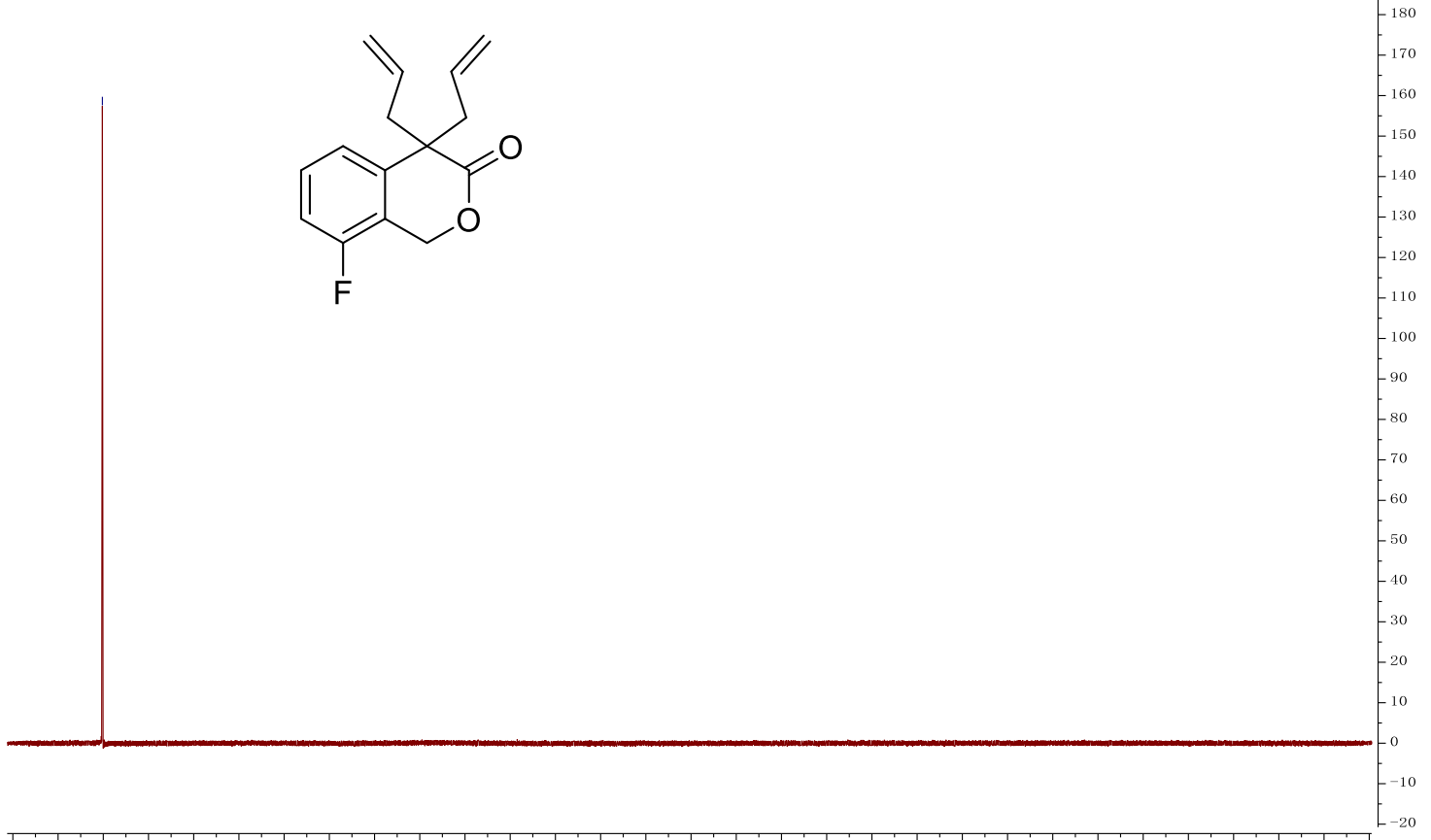
$110-120$
1 
8,8-diallyl-5,8-dihydro-7H-[1,3]dioxolo[4,5-g]isochromen-7-one (10)
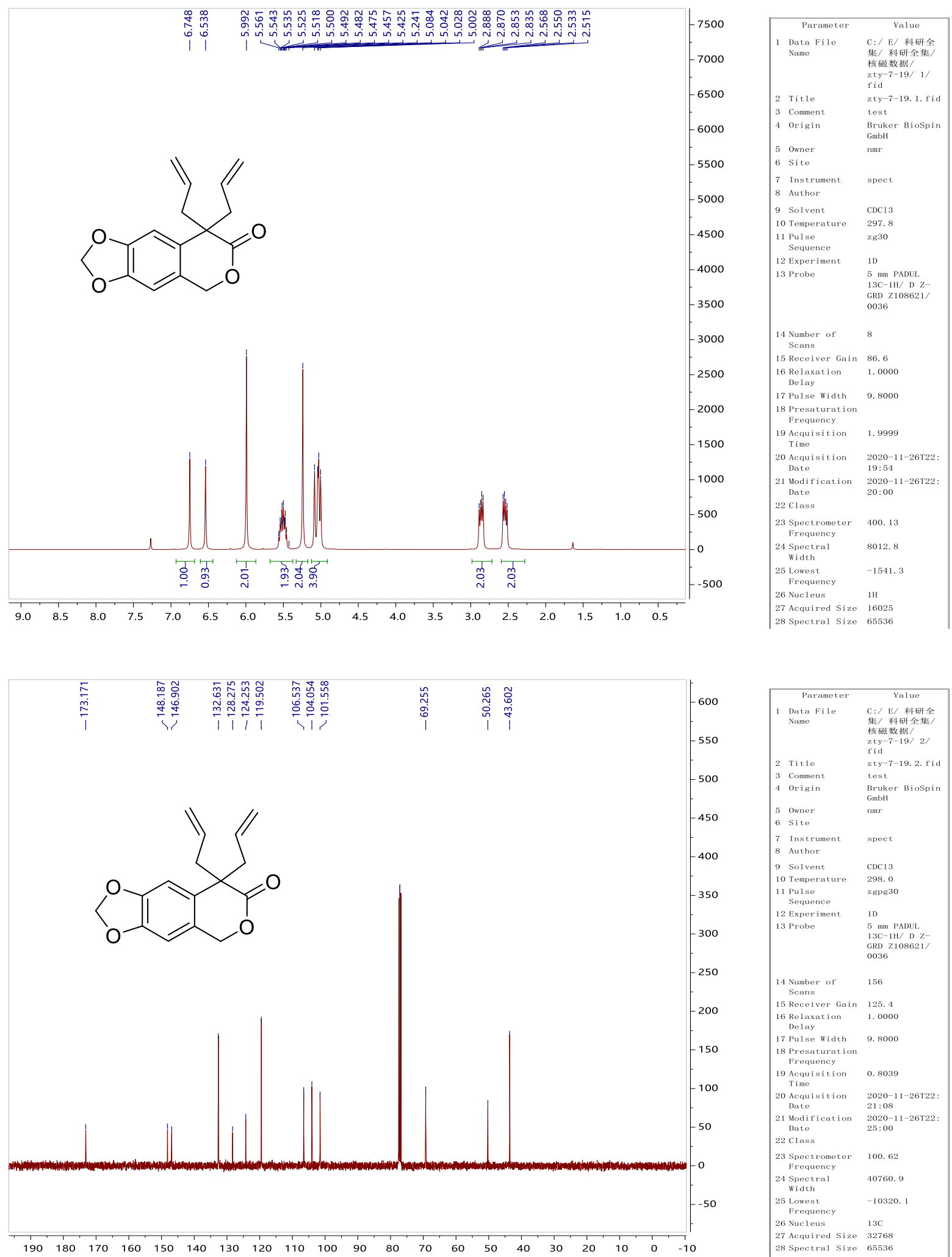
2-allyl-2-(2-(hydroxymethyl)phenyl)pent-4-en-1-ol (1p)
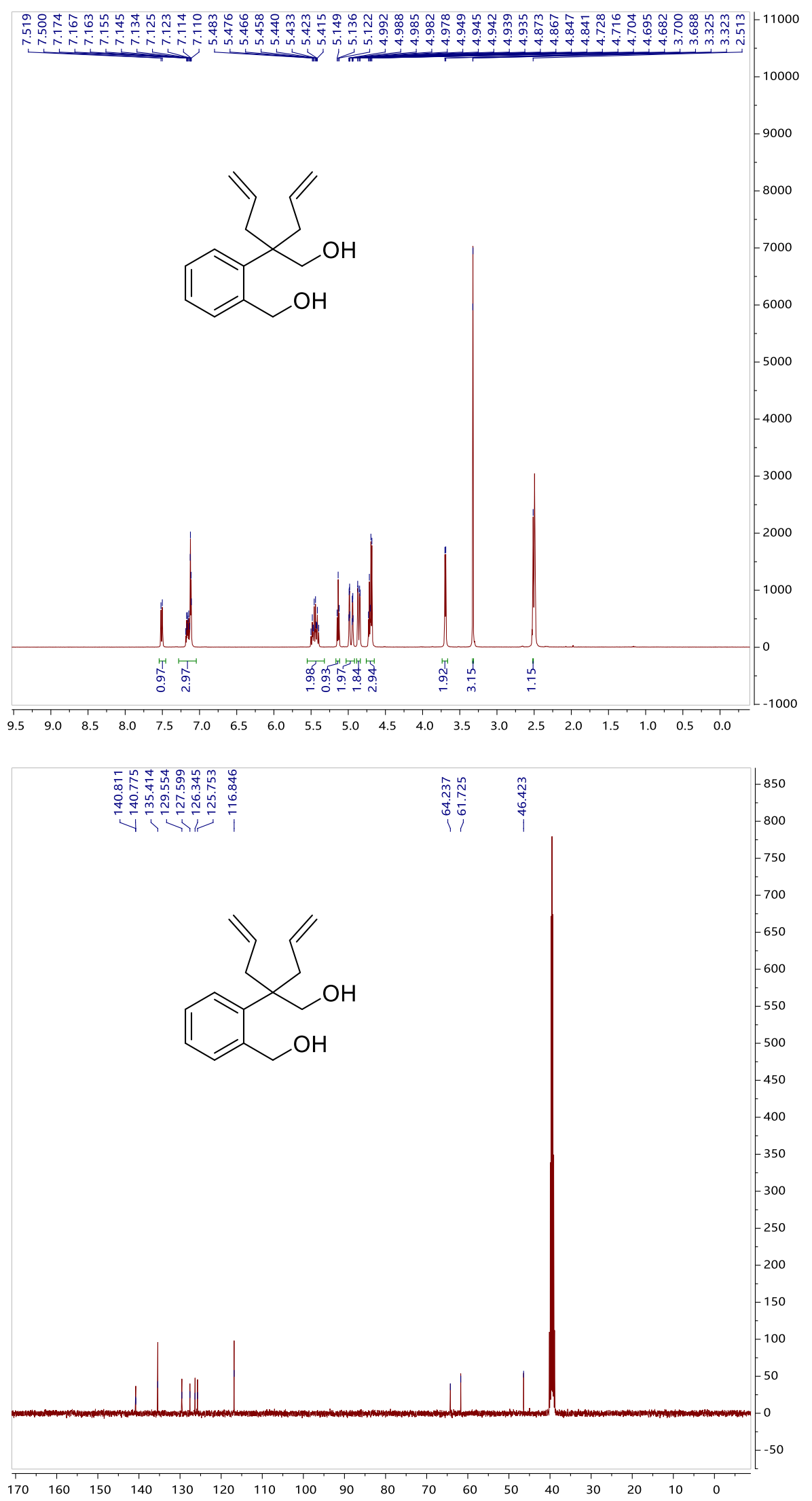
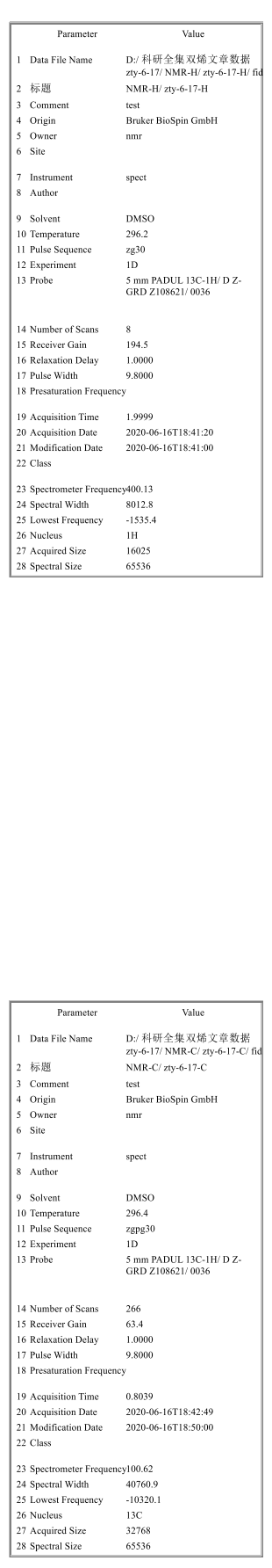
2-allyl-2-(2-(hydroxymethyl)-4-methoxyphenyl)pent-4-en-1-ol (1q)
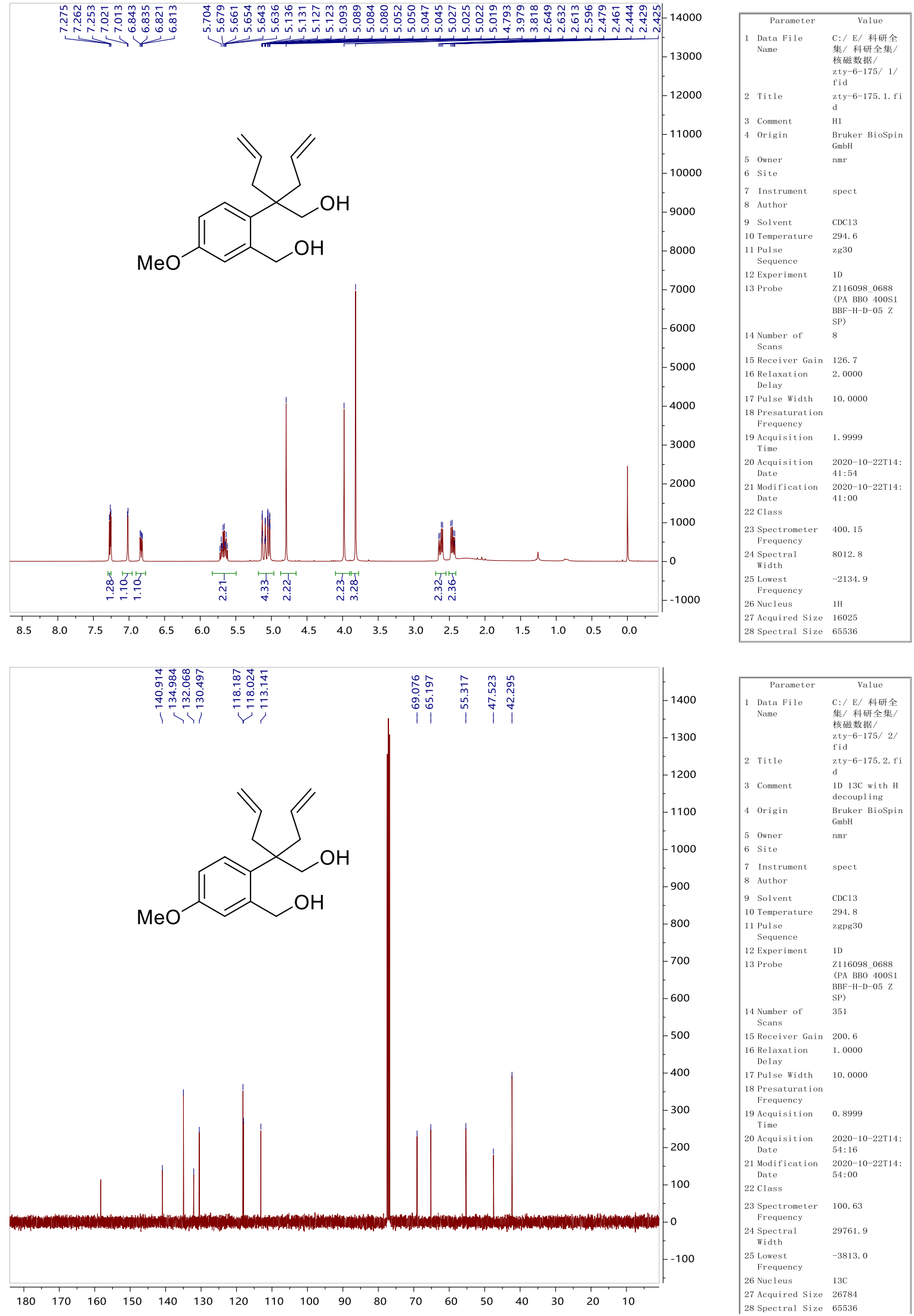


\section{2-allyl-2-(2-(hydroxymethyl)-4-methylphenyl)pent-4-en-1-ol (1r)}
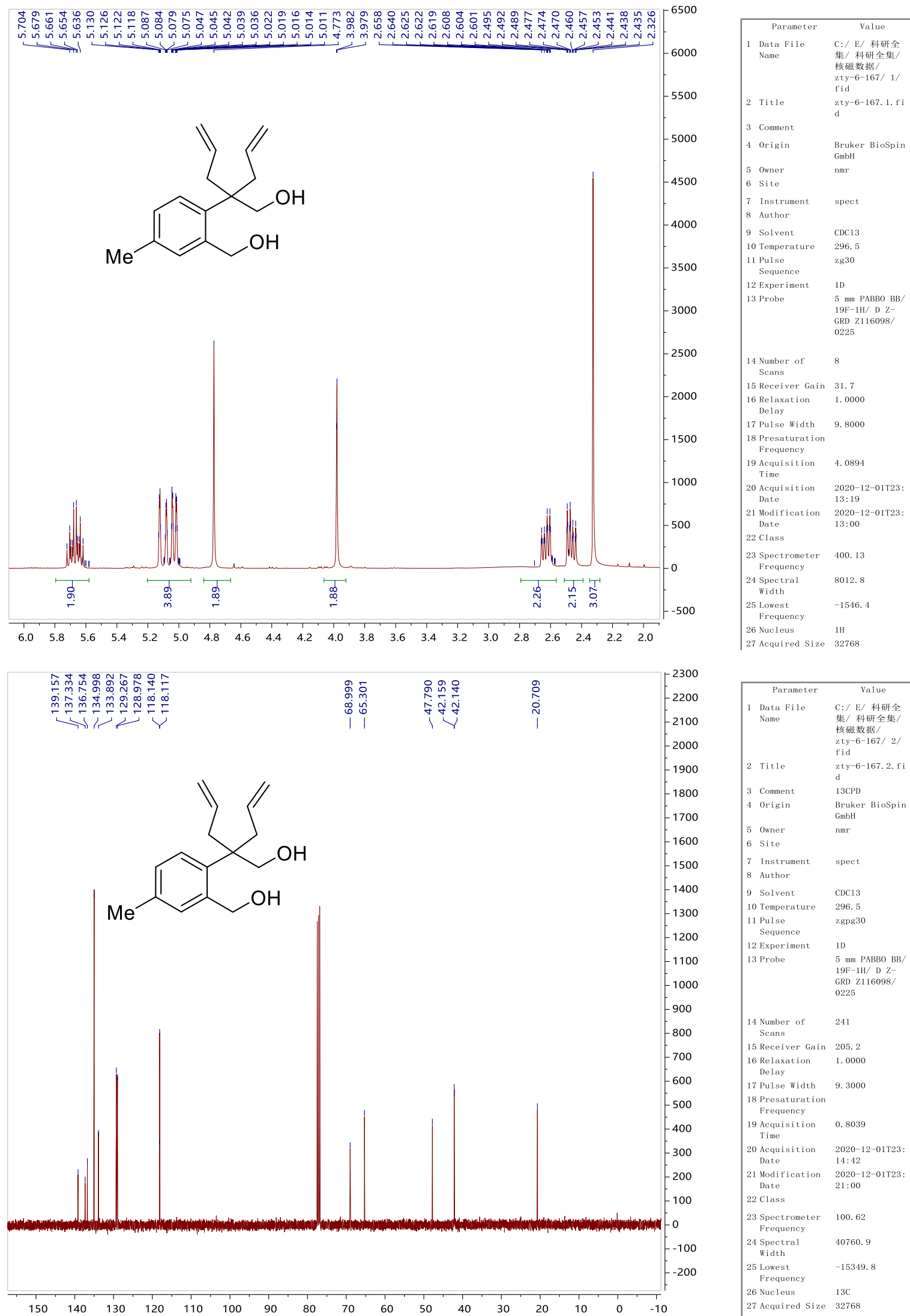

\begin{tabular}{|c|c|}
\hline Parameter & Value \\
\hline $\begin{array}{l}1 \text { Data File } \\
\text { Name }\end{array}$ & $\begin{array}{l}\mathrm{C}: / \mathrm{E} / \text { 科研全 } \\
\text { 集/科研全集/ } \\
\text { 核磁数据/ } \\
\text { zty } 6-167 / 2 / \\
\text { fid }\end{array}$ \\
\hline 2 Title & $\begin{array}{l}\mathrm{zty}-6-167.2 . \mathrm{fi} \\
\mathrm{d}\end{array}$ \\
\hline 3 Comment & $13 \mathrm{CPD}$ \\
\hline 4 Origin & $\begin{array}{l}\text { Bruker BioSpin } \\
\text { GmbH }\end{array}$ \\
\hline $\begin{array}{ll}5 & \text { Owner } \\
6 & \text { Site }\end{array}$ & $\mathrm{nmr}$ \\
\hline $\begin{array}{ll}7 & \text { Instrument } \\
8 & \text { Author }\end{array}$ & spect \\
\hline 9 Solvent & $\mathrm{CDC} 13$ \\
\hline 10 Temperature & 296.5 \\
\hline $\begin{array}{l}11 \text { Pulse } \\
\text { Sequence }\end{array}$ & zgpg30 \\
\hline 12 Experiment & $1 \mathrm{D}$ \\
\hline 13 Probe & $\begin{array}{l}5 \mathrm{~mm} P \mathrm{PABBO} \mathrm{BB} / \\
19 \mathrm{~F}-1 \mathrm{H} / \mathrm{D} \mathrm{Z}- \\
\mathrm{GRD} Z \mathrm{Z116098} \\
0225\end{array}$ \\
\hline $\begin{array}{l}14 \text { Number of } \\
\text { Scans }\end{array}$ & 241 \\
\hline 15 Receiver Gain & 205.2 \\
\hline $\begin{array}{l}16 \text { Relaxation } \\
\text { Delay }\end{array}$ & 1. 0000 \\
\hline 17 Pulse width & 9. 3000 \\
\hline $\begin{array}{l}18 \text { Presaturation } \\
\text { Frequency }\end{array}$ & \\
\hline $\begin{array}{l}19 \text { Acquisition } \\
\text { Time }\end{array}$ & 0.8039 \\
\hline $\begin{array}{l}20 \text { Acquisition } \\
\text { Date }\end{array}$ & $\begin{array}{l}2020-12-01 T 23: \\
14: 42\end{array}$ \\
\hline $\begin{array}{l}21 \text { Modification } \\
\text { Date } \\
22 \text { Class }\end{array}$ & $\begin{array}{l}\text { 2020-12-01T23: } \\
21: 00\end{array}$ \\
\hline $\begin{array}{l}23 \text { Spectrometer } \\
\text { Frequency }\end{array}$ & 100.62 \\
\hline $\begin{array}{l}24 \text { Spectral } \\
\text { Width }\end{array}$ & 40760.9 \\
\hline $\begin{array}{l}25 \text { Lowest } \\
\text { Frequency }\end{array}$ & -15349.8 \\
\hline 26 Nucleus & $13 \mathrm{C}$ \\
\hline 27 Acquired Size & $\begin{array}{l}32768 \\
65536\end{array}$ \\
\hline
\end{tabular}




\section{2-allyl-2-(3-fluoro-2-(hydroxymethyl)phenyl)pent-4-en-1-ol (1s)}
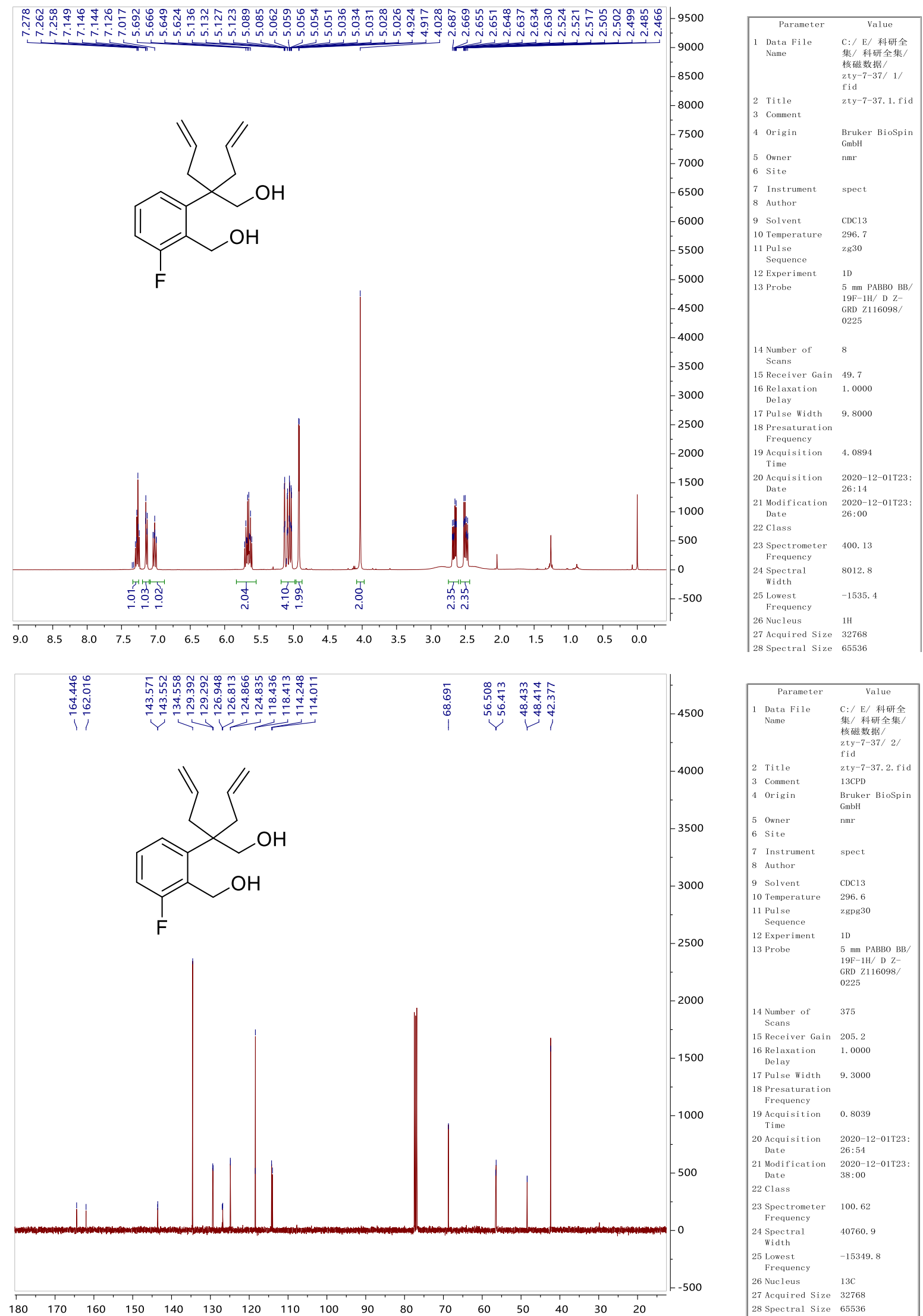

\begin{tabular}{|c|c|}
\hline Parameter & Value \\
\hline $\begin{array}{l}1 \text { Data File } \\
\text { Name }\end{array}$ & $\begin{array}{l}\mathrm{C}: / \mathrm{E} / \text { 科研全 } \\
\text { 集/ 科研全集/ } \\
\text { 核磁数据// } \\
\text { zty-77/ } \\
\text { fid }\end{array}$ \\
\hline 2 Title & zty-7-37. 2. fid \\
\hline 3 Comment & $13 \mathrm{CPD}$ \\
\hline 4 Origin & $\begin{array}{l}\text { Bruker BioSpin } \\
\text { GmbH }\end{array}$ \\
\hline $\begin{array}{ll}5 & \text { Owner } \\
6 & \text { Site }\end{array}$ & $\mathrm{nmr}$ \\
\hline $\begin{array}{ll}7 & \text { Instrument } \\
8 & \text { Author }\end{array}$ & spect \\
\hline 9 Solvent & $\mathrm{CDC13}$ \\
\hline 10 Temperature & 296.6 \\
\hline $\begin{array}{l}11 \text { Pulse } \\
\text { Sequence }\end{array}$ & zgpg30 \\
\hline 12 Experiment & $1 \mathrm{D}$ \\
\hline 13 Probe & $\begin{array}{l}5 \mathrm{~mm} \text { PABBO BB/ } \\
19 \mathrm{~F}-1 \mathrm{H} / \mathrm{D} \text { Z - } \\
\text { GRD Z116098/ } \\
0225\end{array}$ \\
\hline $\begin{array}{l}14 \text { Number of } \\
\text { Scans }\end{array}$ & 375 \\
\hline 15 Receiver Gain & 205.2 \\
\hline $\begin{array}{l}16 \text { Relaxation } \\
\text { Delay }\end{array}$ & 1. 0000 \\
\hline 17 Pulse Width & 9. 3000 \\
\hline $\begin{array}{l}18 \text { Presaturation } \\
\text { Frequency }\end{array}$ & \\
\hline $\begin{array}{l}19 \text { Acquisition } \\
\text { Time }\end{array}$ & 0.8039 \\
\hline $\begin{array}{l}20 \text { Acquisition } \\
\text { Date }\end{array}$ & $\begin{array}{l}2020-12-01 T 23: \\
26: 54\end{array}$ \\
\hline $\begin{array}{l}21 \text { Modification } \\
\text { Date }\end{array}$ & $\begin{array}{l}2020-12-01 \mathrm{~T} 23: \\
38: 00\end{array}$ \\
\hline 22 Class & \\
\hline $\begin{array}{l}23 \text { Spectrometer } \\
\text { Frequency }\end{array}$ & 100.62 \\
\hline $\begin{array}{l}24 \text { Spectral } \\
\text { Width }\end{array}$ & 40760.9 \\
\hline $\begin{array}{l}25 \text { Lowest } \\
\text { Frequency }\end{array}$ & -15349.8 \\
\hline 26 Nucleus & $13 \mathrm{C}$ \\
\hline 27 Acquired Size & 32768 \\
\hline 28 Spectral Size & 65536 \\
\hline
\end{tabular}



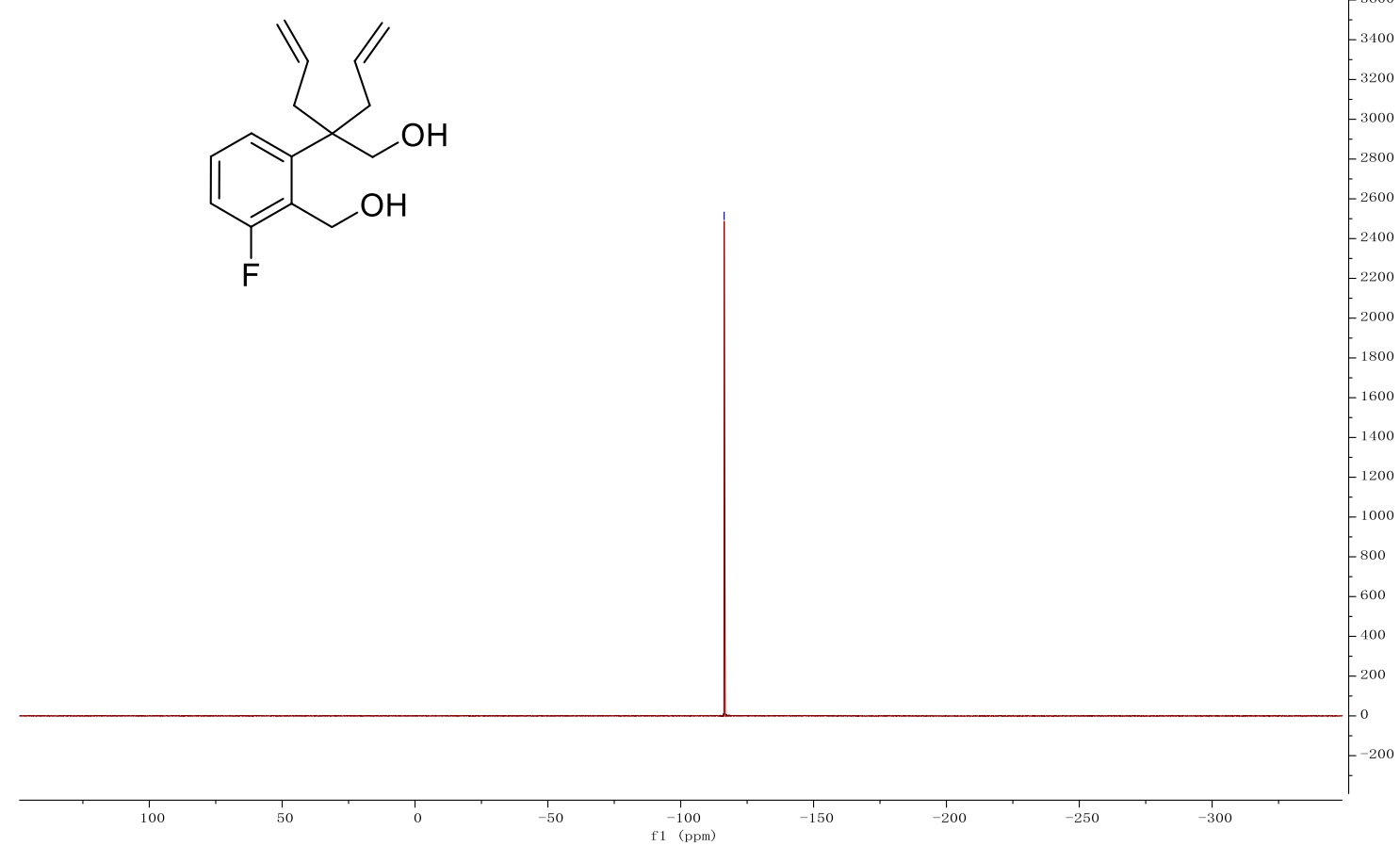
methyl 2-allyl-2-phenylpent-4-enoate (1t)
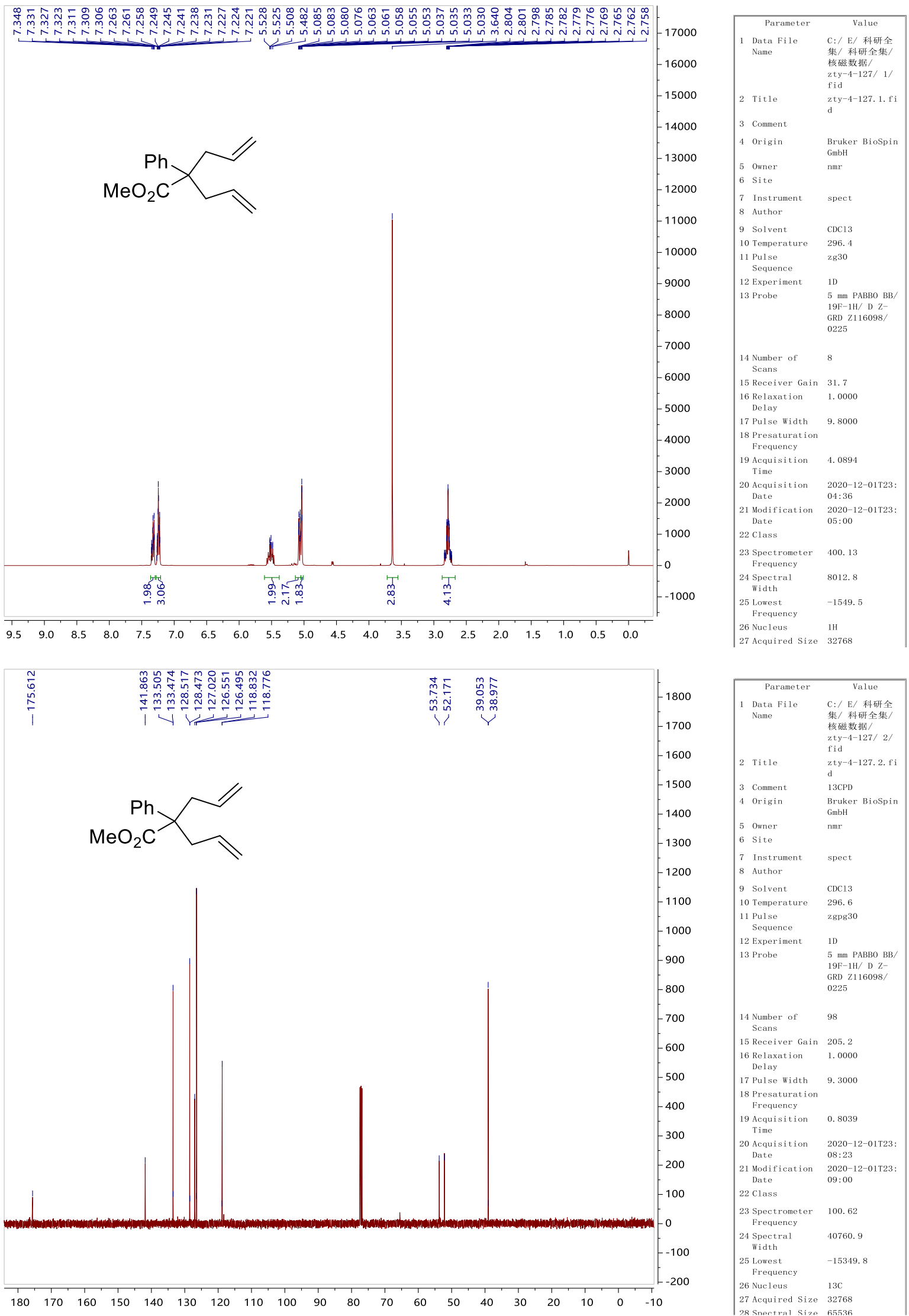


\section{3,3-diallylbenzofuran-2(3H)-one (1u)}

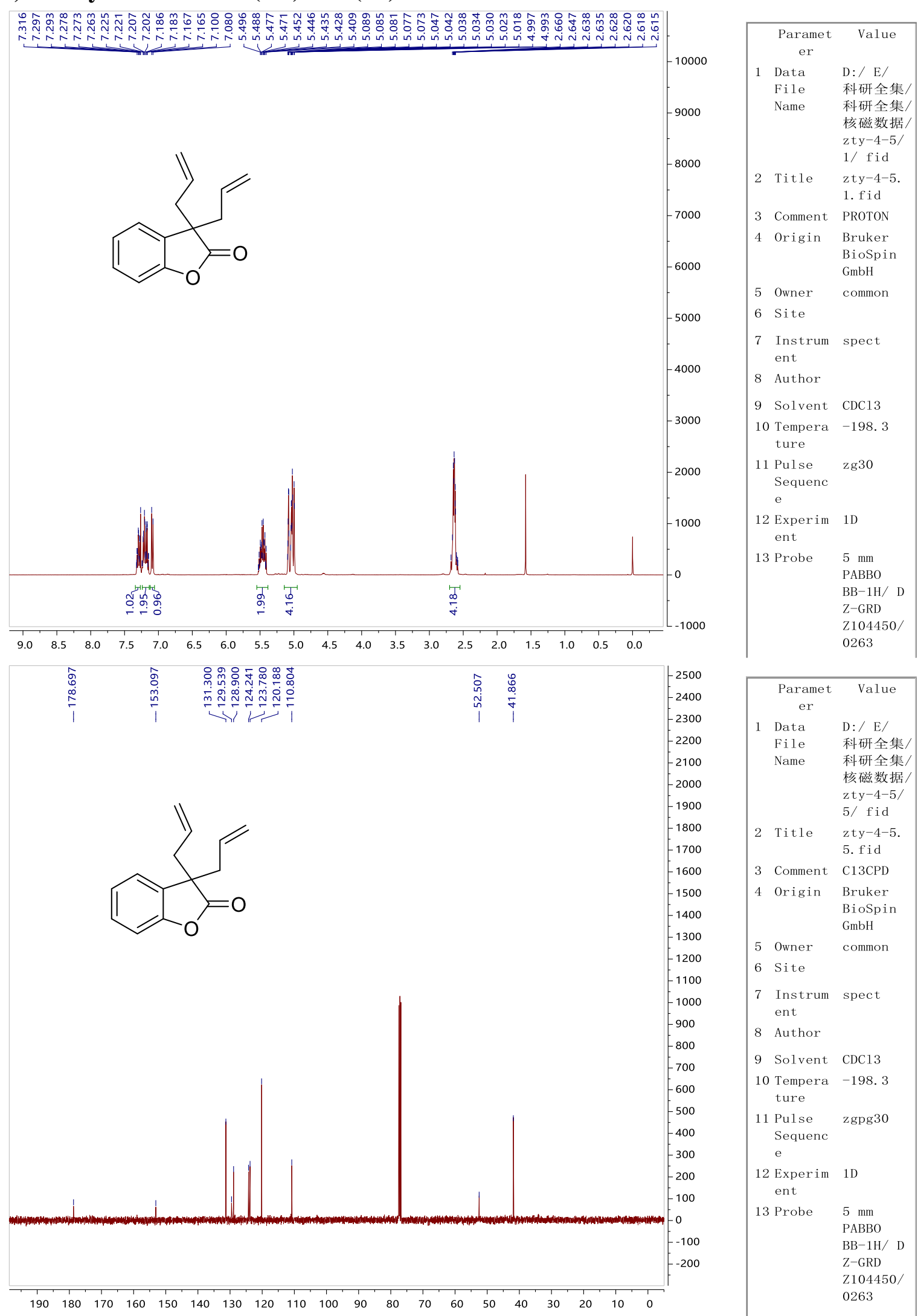




\section{3,3-diallyl-1-methylindolin-2-one (1v)}

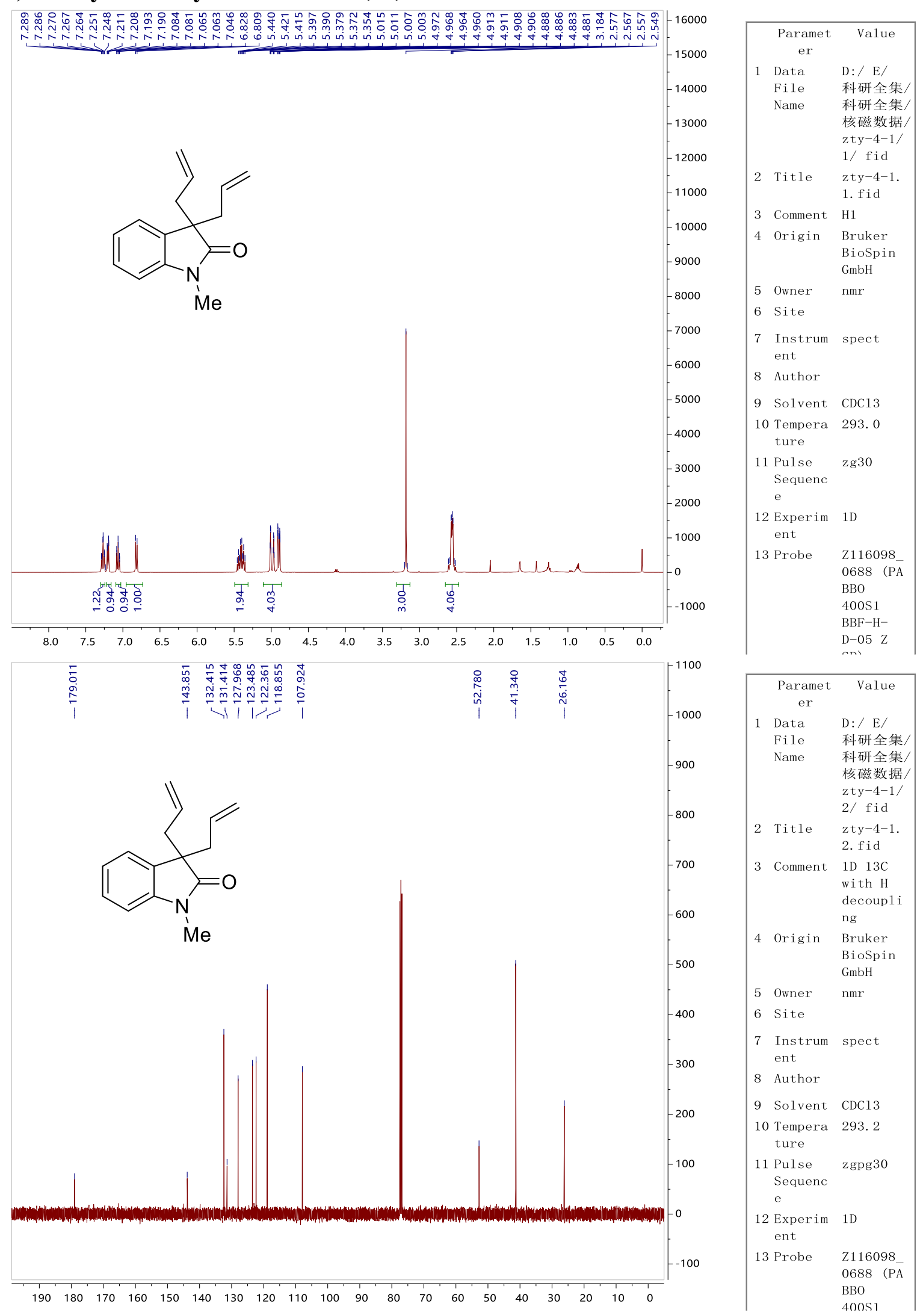




\section{3-methyl-4-methylenespiro[cyclopentane-1,4'-isochroman]-3'-one (2a)}
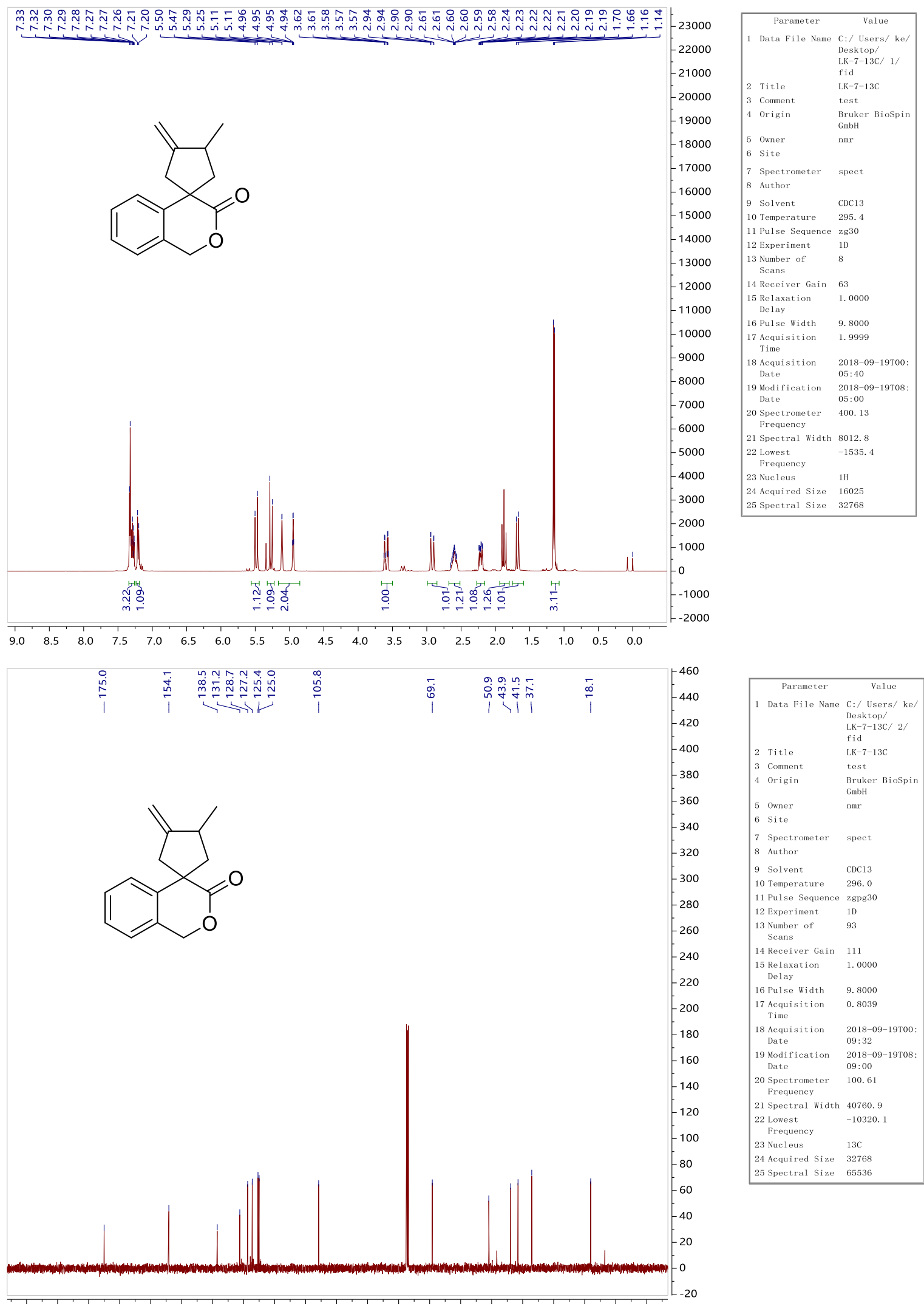

$\begin{array}{lllllllllllllllllllll}200 & 190 & 180 & 170 & 160 & 150 & 140 & 130 & 120 & 110 & 100 & 90 & 80 & 70 & 60 & 50 & 40 & 30 & 20 & 10 & 0\end{array}$ 
3,7'-dimethyl-4-methylenespiro[cyclopentane-1,4'-isochroman]-3'-one (2b)
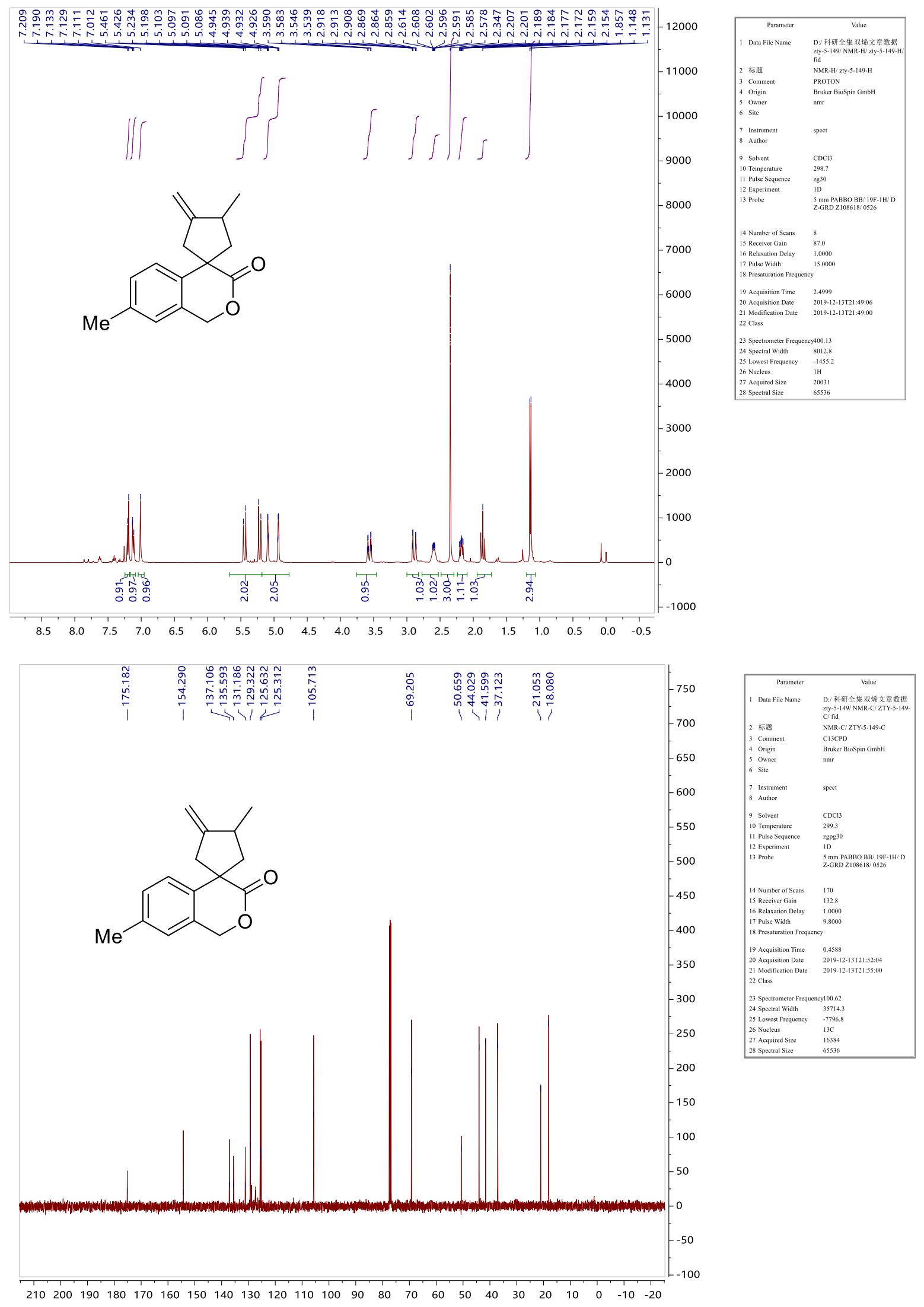
7'-chloro-3-methyl-4-methylenespiro[cyclopentane-1,4'-isochroman]-3'-one (2c)
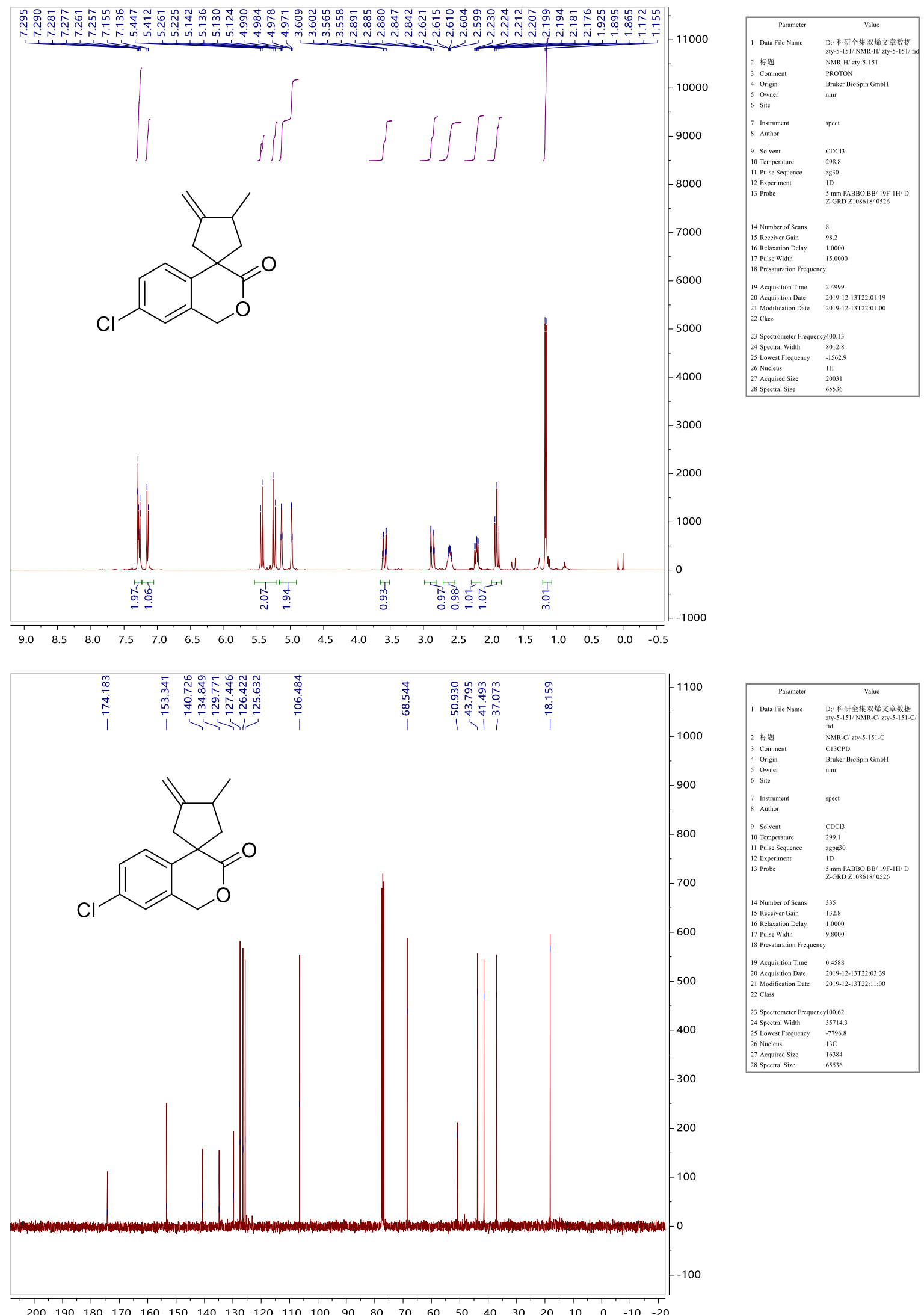

$\begin{array}{lllllllllllllllllllllll}200 & 190 & 180 & 170 & 160 & 150 & 140 & 130 & 120 & 110 & 100 & 90 & 80 & 70 & 60 & 50 & 40 & 30 & 20 & 10 & 0 & -10 & -20\end{array}$ 
7'-methoxy-3-methyl-4-methylenespiro[cyclopentane-1,4'-isochroman]-3'-one (2d)
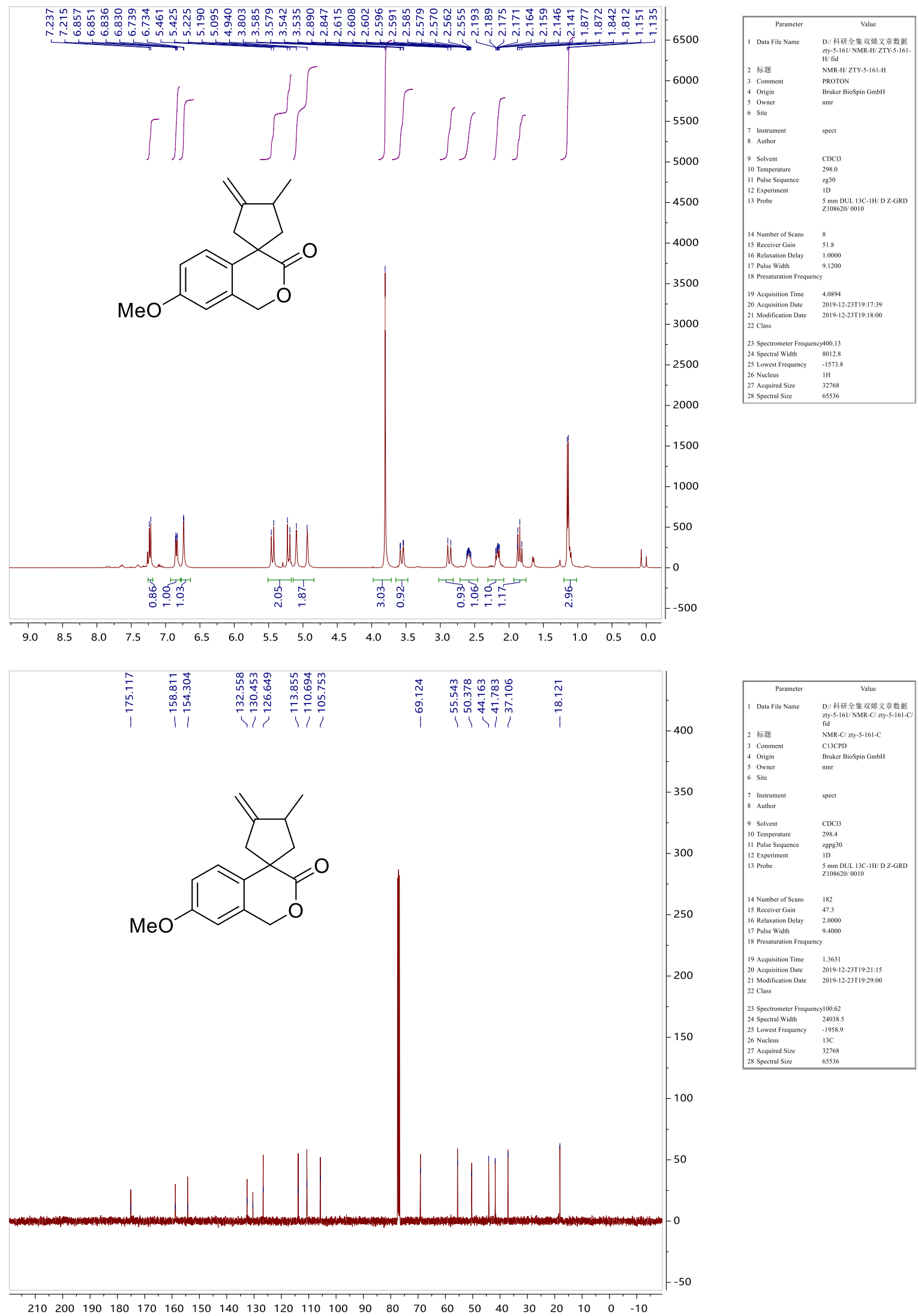
3,6'-dimethyl-4-methylenespiro[cyclopentane-1,4'-isochroman]-3'-one (2e)

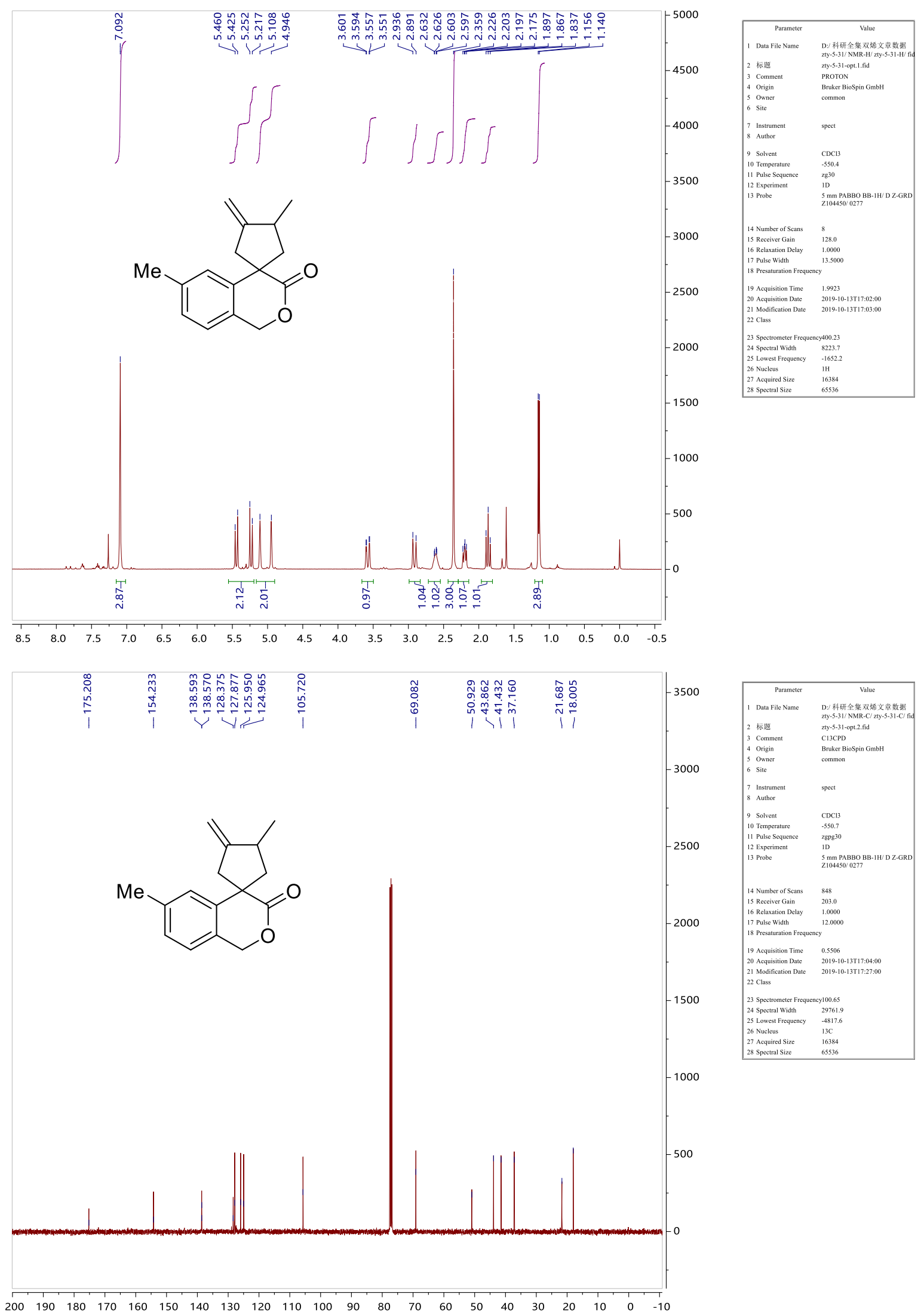


6'-chloro-3-methyl-4-methylenespiro[cyclopentane-1,4'-isochroman]-3'-one (2f)

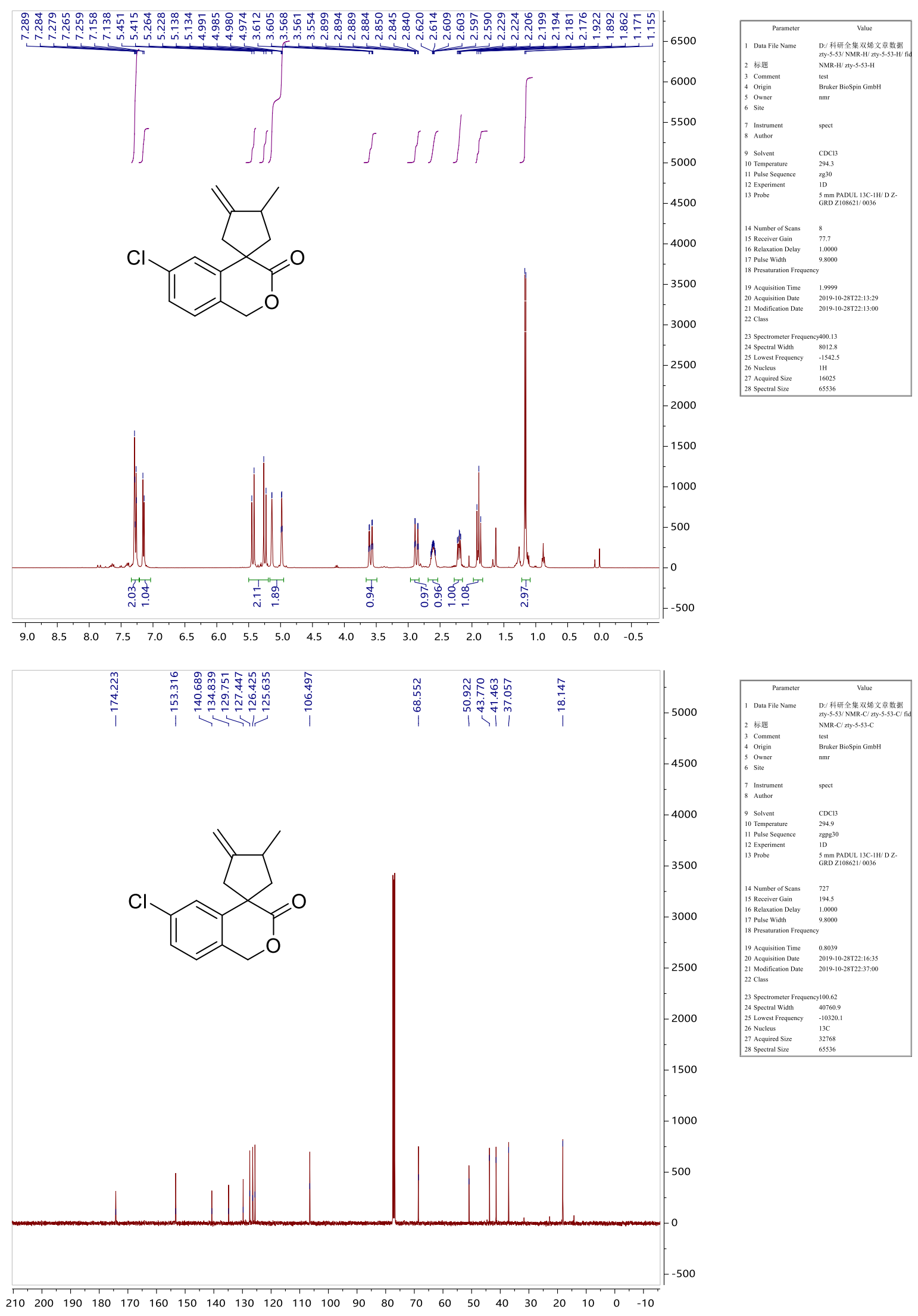


6'-methoxy-3-methyl-4-methylenespiro[cyclopentane-1,4'-isochroman]-3'-one (2g)
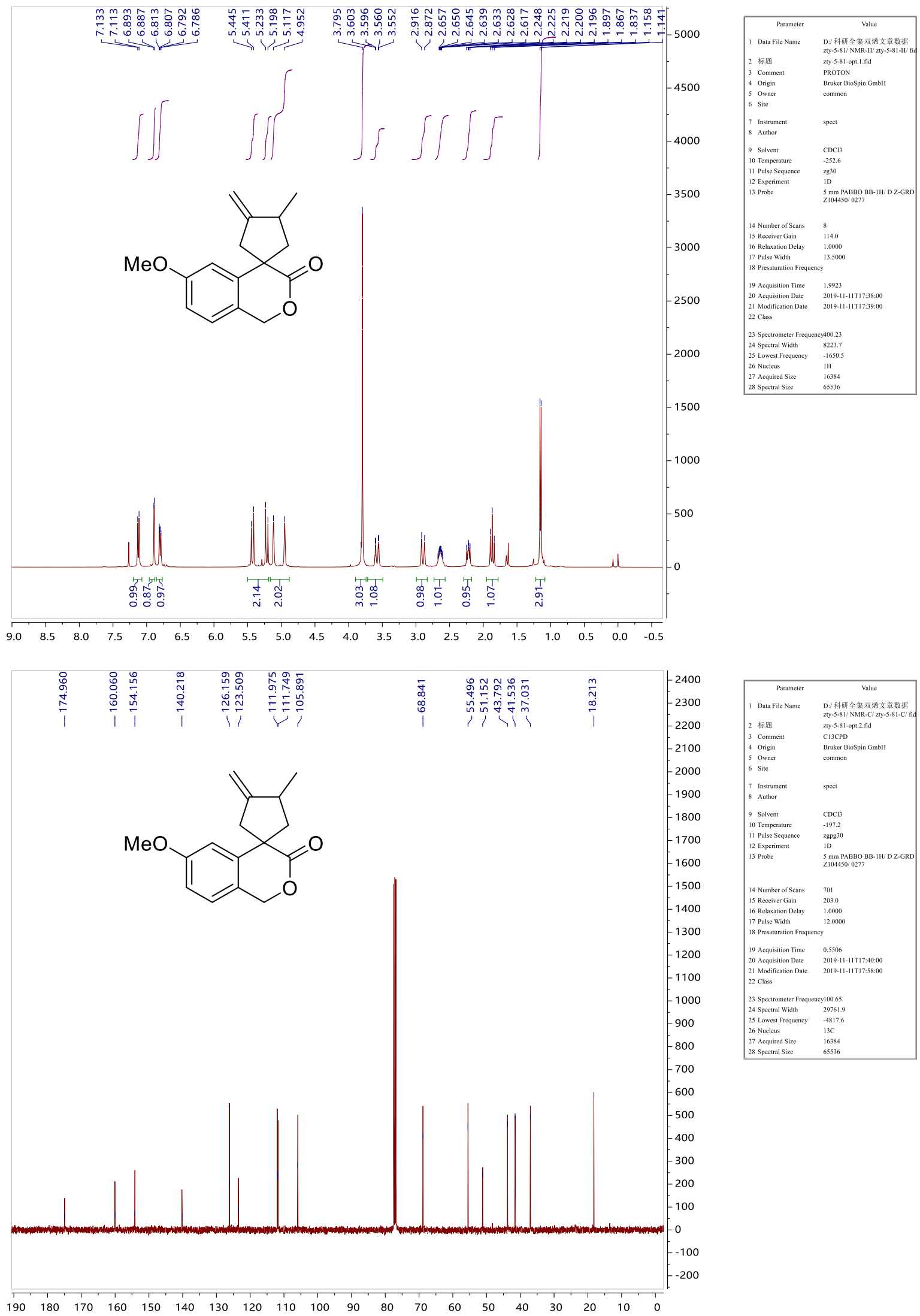
6'-bromo-3-methyl-4-methylenespiro[cyclopentane-1,4'-isochroman]-3'-one (2h)
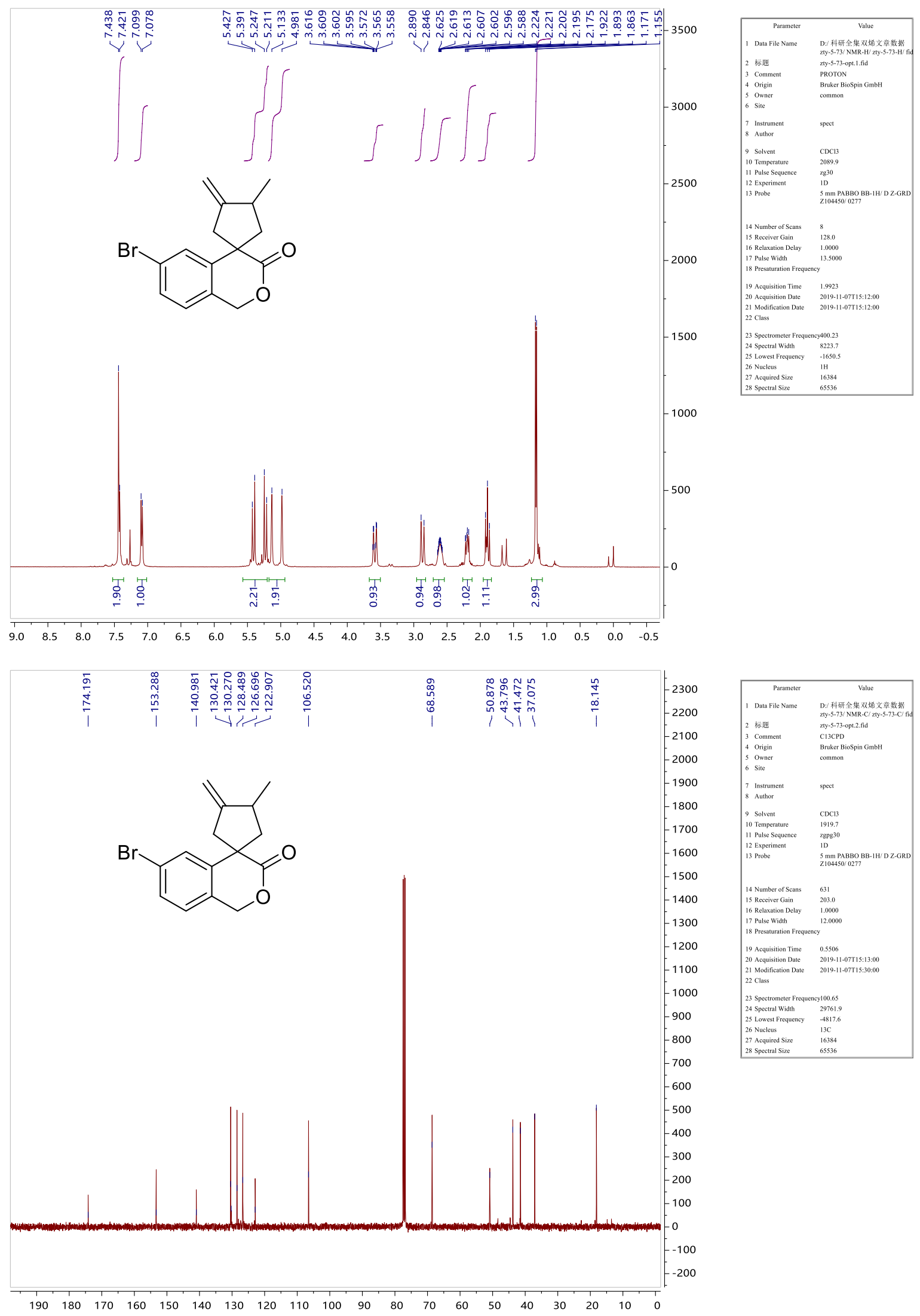
6'-fluoro-3-methyl-4-methylenespiro[cyclopentane-1,4'-isochroman]-3'-one (2i)
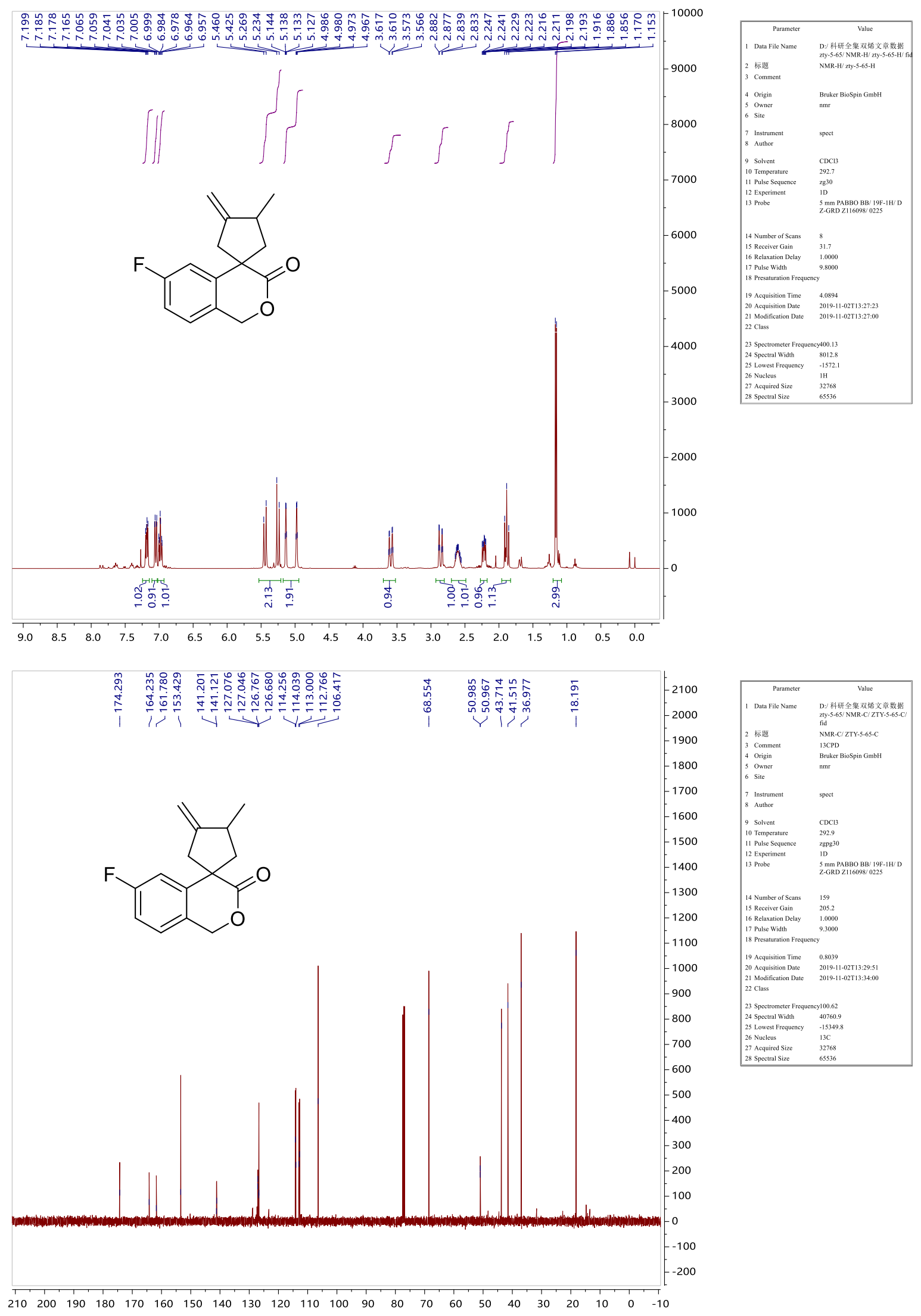


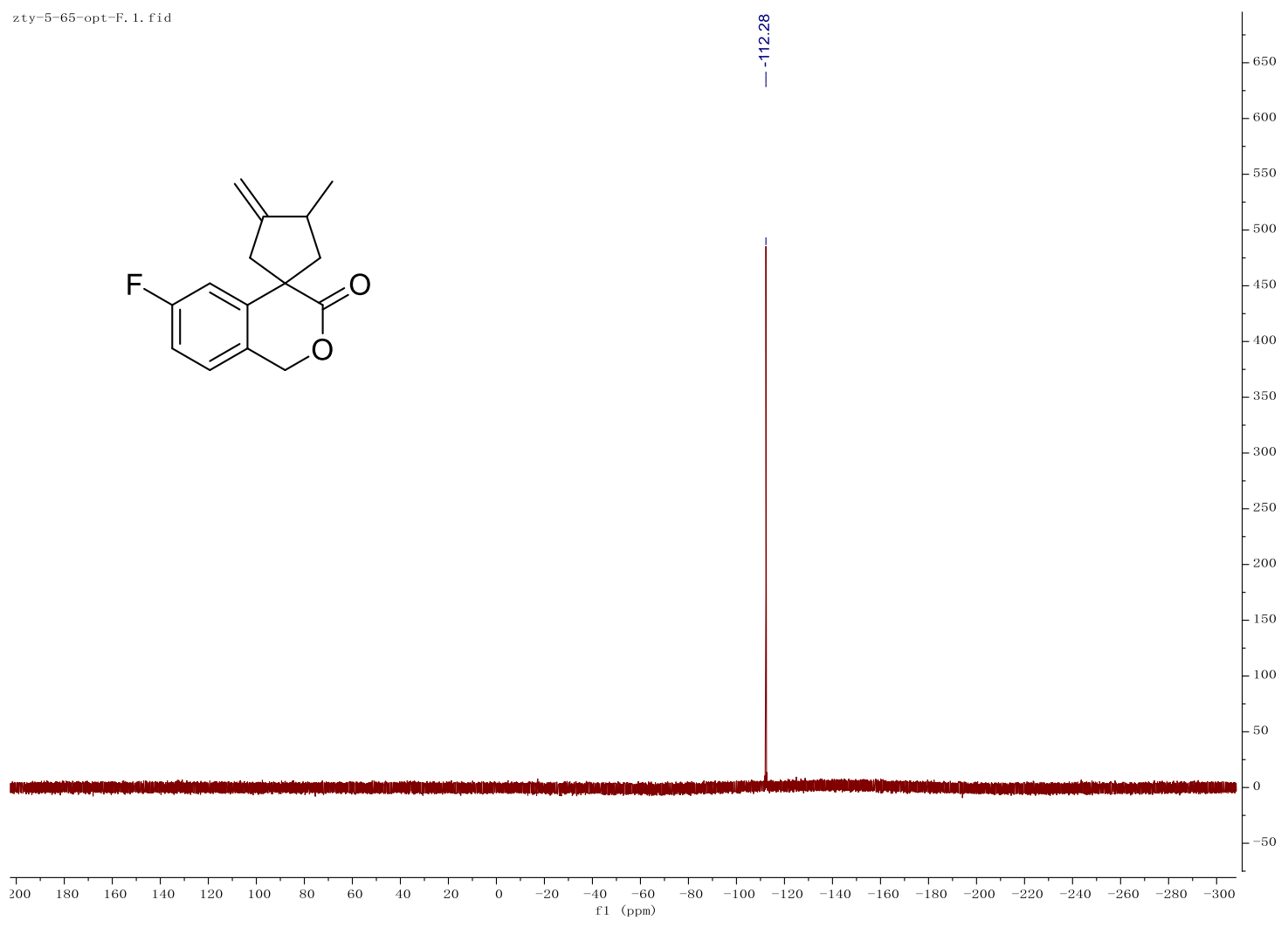


3-methyl-4-methylene-6' -(trifluoromethyl)spiro[cyclopentane-1,4'-isochroman]-3'-one $(2 \mathbf{j})$

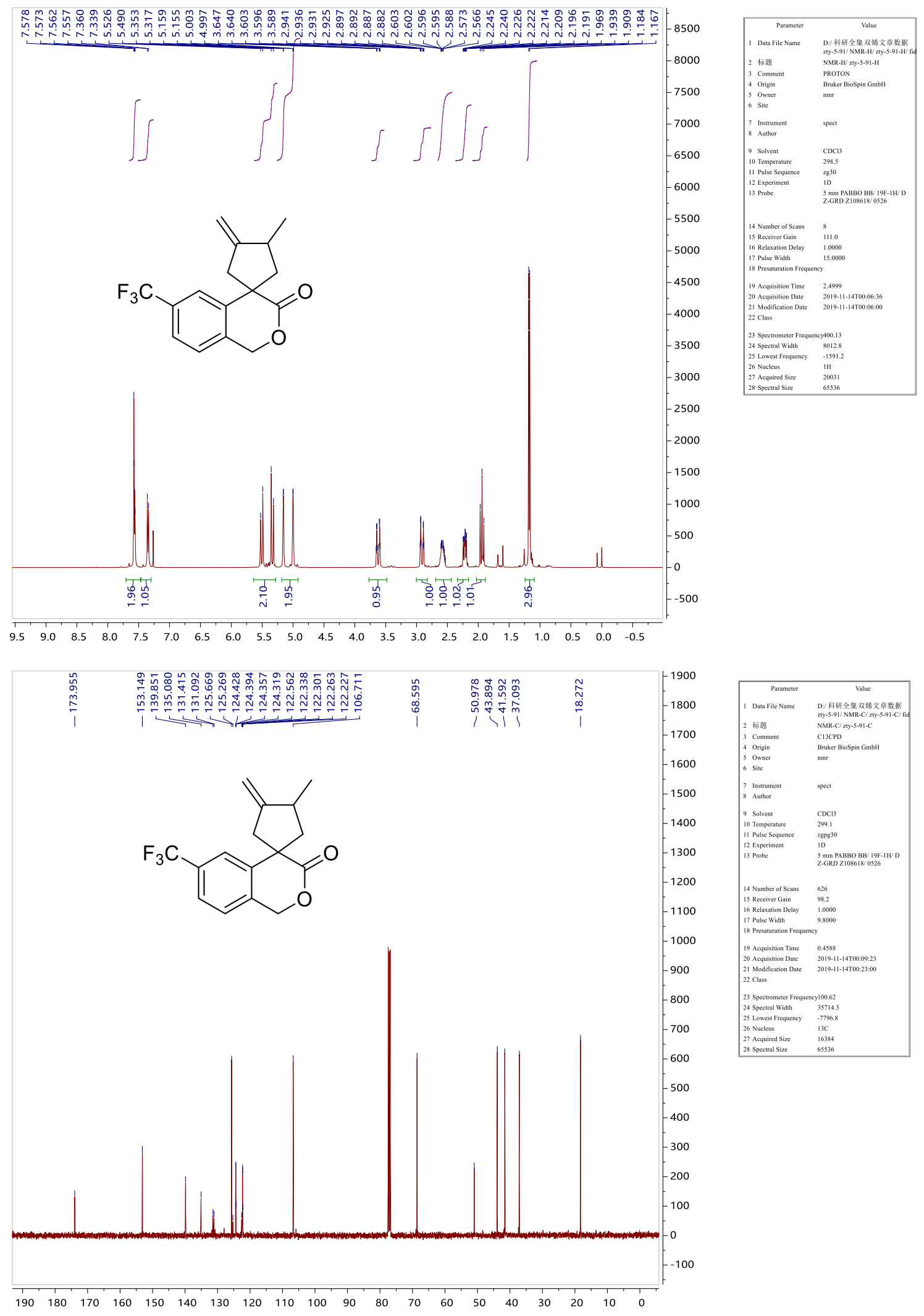




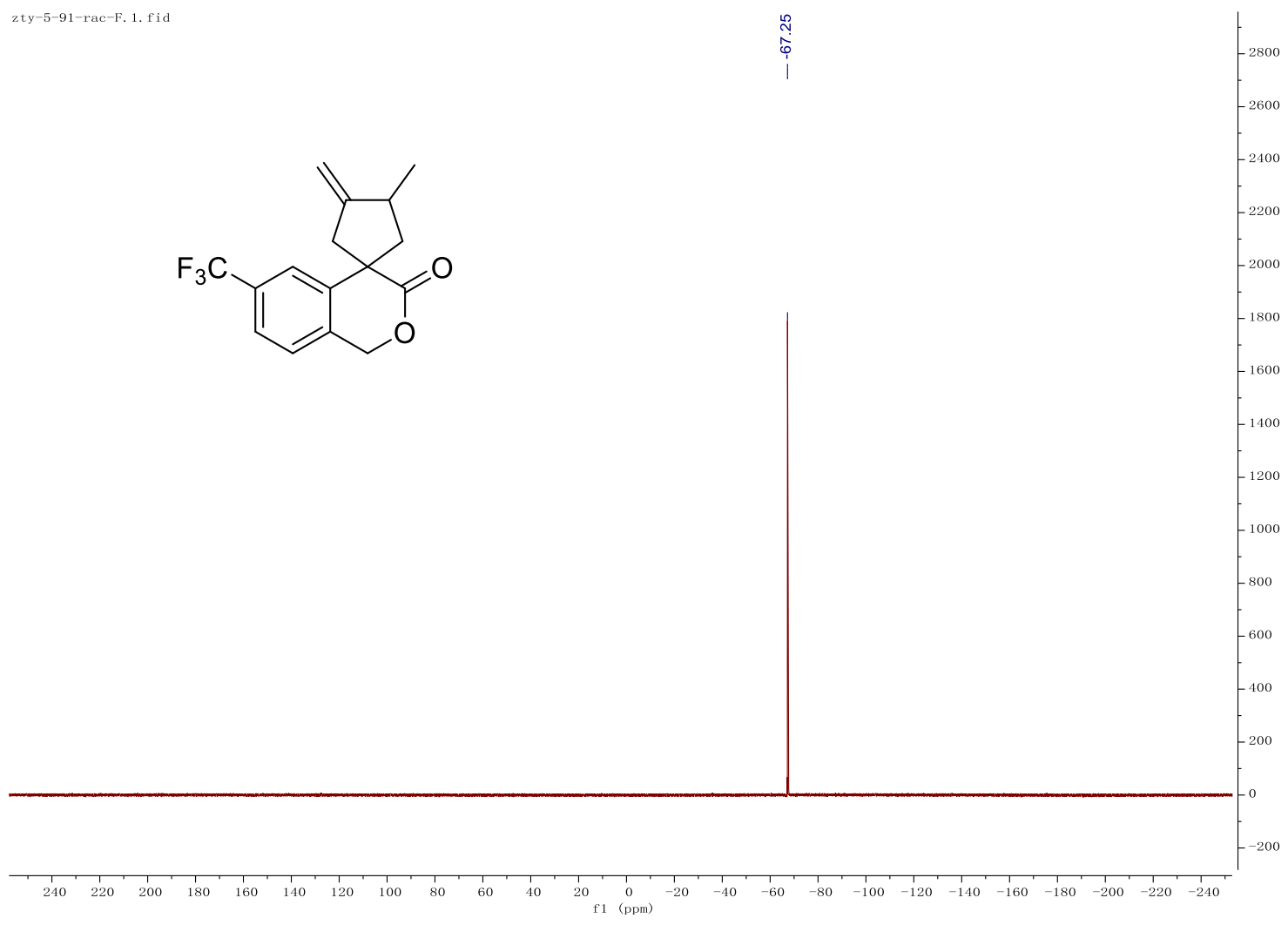


3-methyl-4-methylene-6' -(4-nitrophenyl)spiro[cyclopentane-1,4'-isochroman]-3'-one (2k)
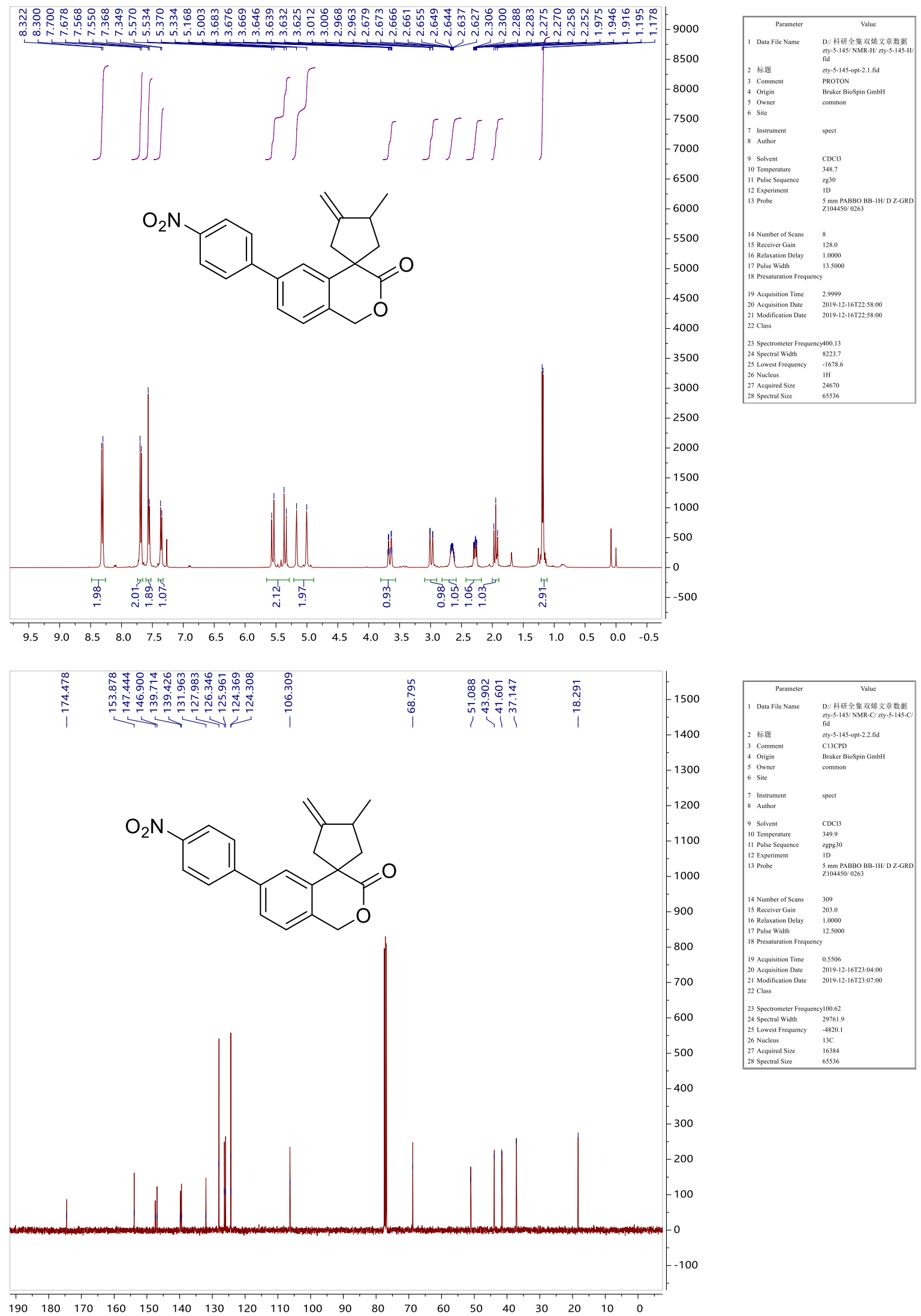
methyl 4-(3-methyl-4-methylene-3'-oxospiro[cyclopentane-1,4'-isochroman]-6' yl)benzoate (2l)
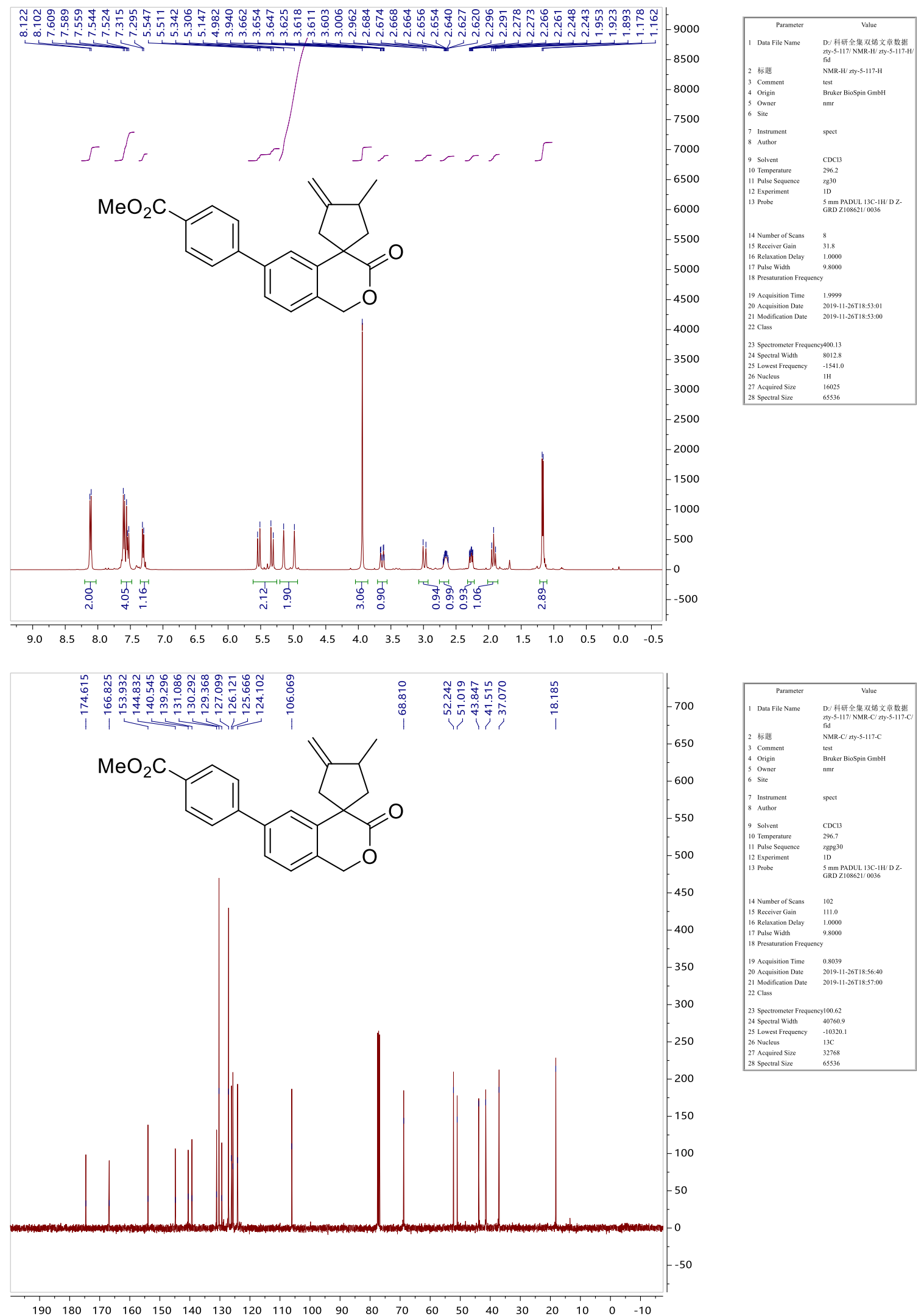
8'-methyl-4-methylenespiro[cyclopentane-1,4'-isochroman]-3'-one (2m)
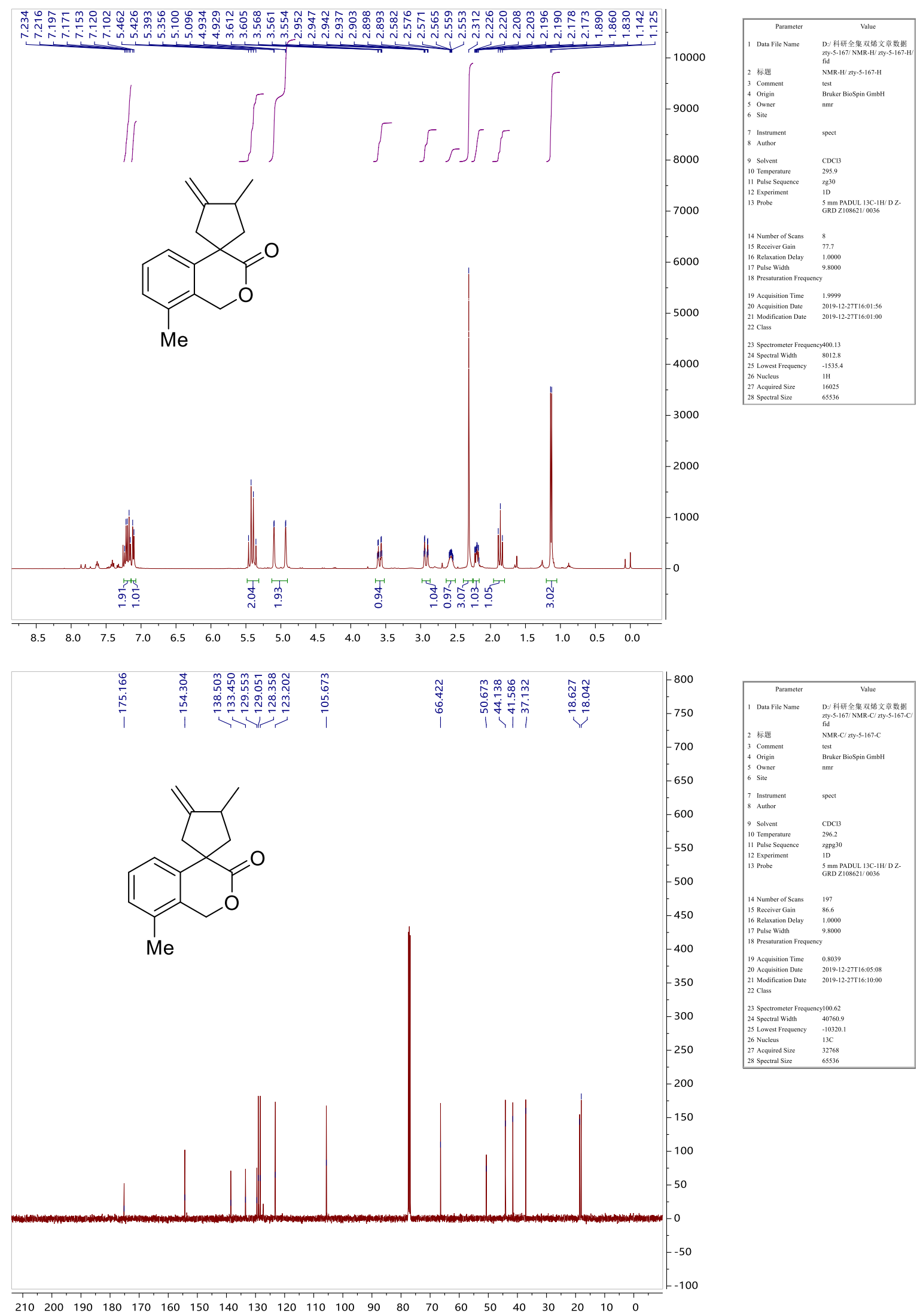
8'-fluoro-3-methyl-4-methylenespiro[cyclopentane-1,4'-isochroman]-3' -one (2n)
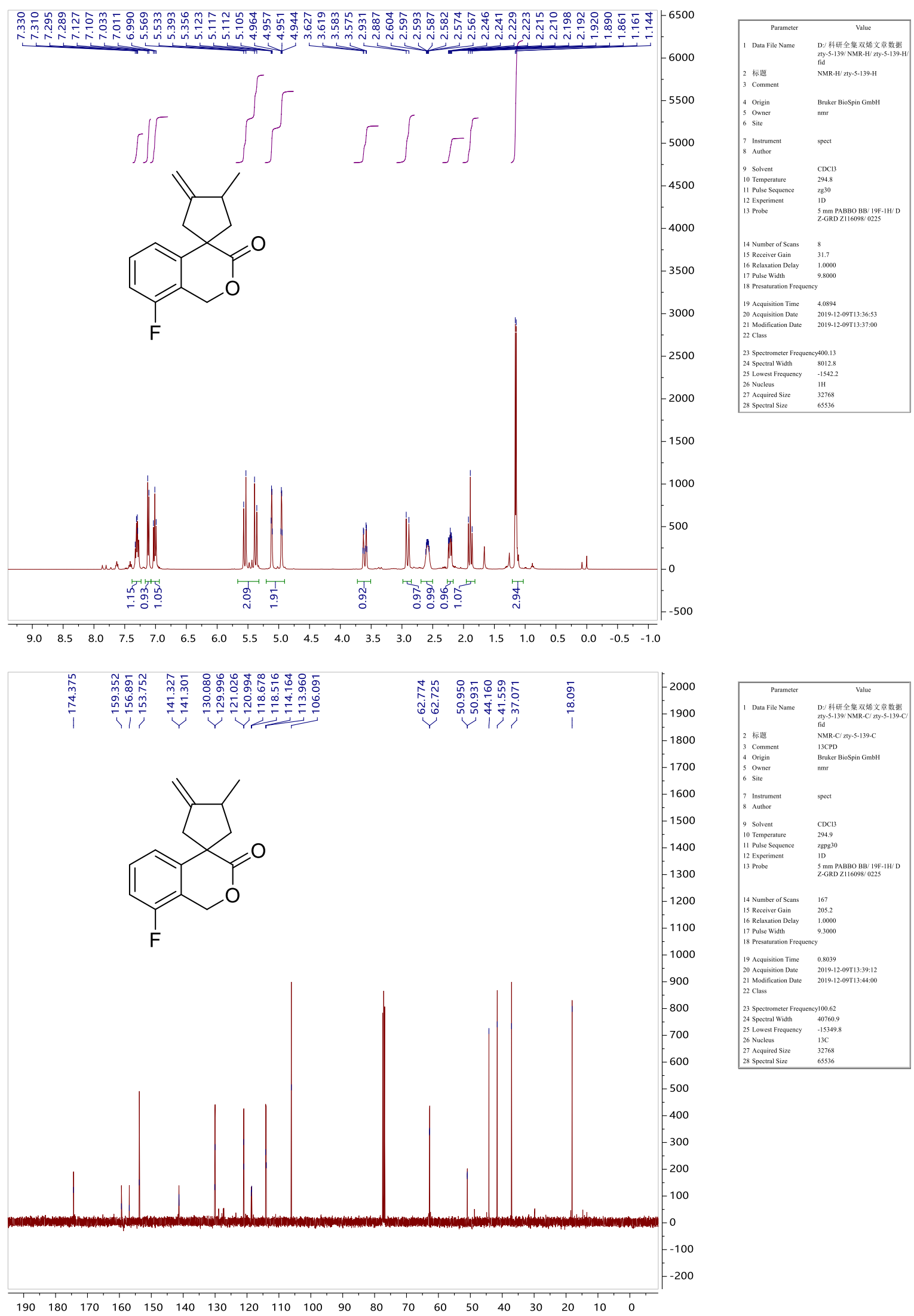


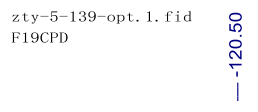
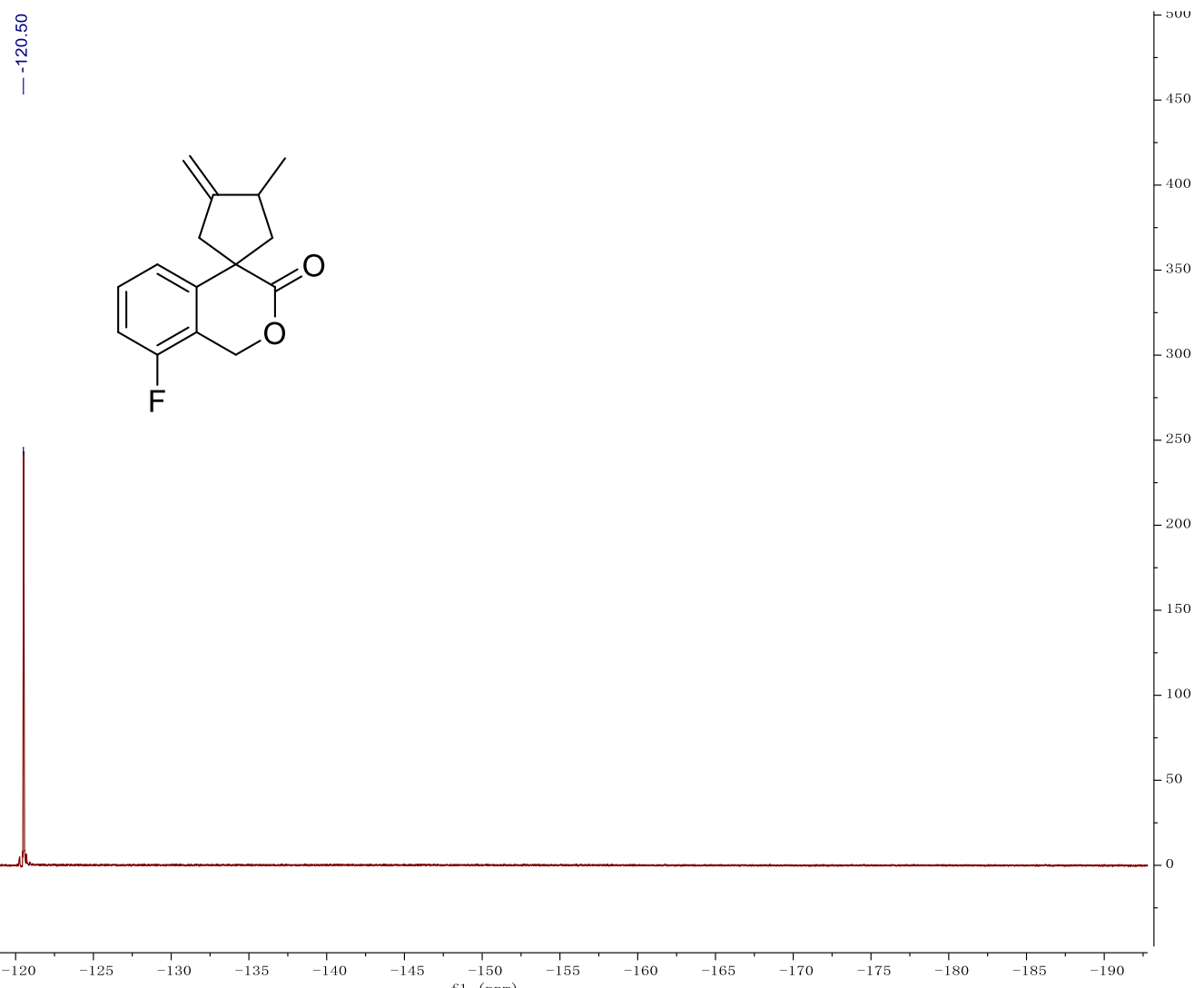
3-methyl-4-methylene-5'H,7'H-spiro[cyclopentane-1,8'-[1,3]dioxolo[4,5-g]isochromen]7'-one (20)
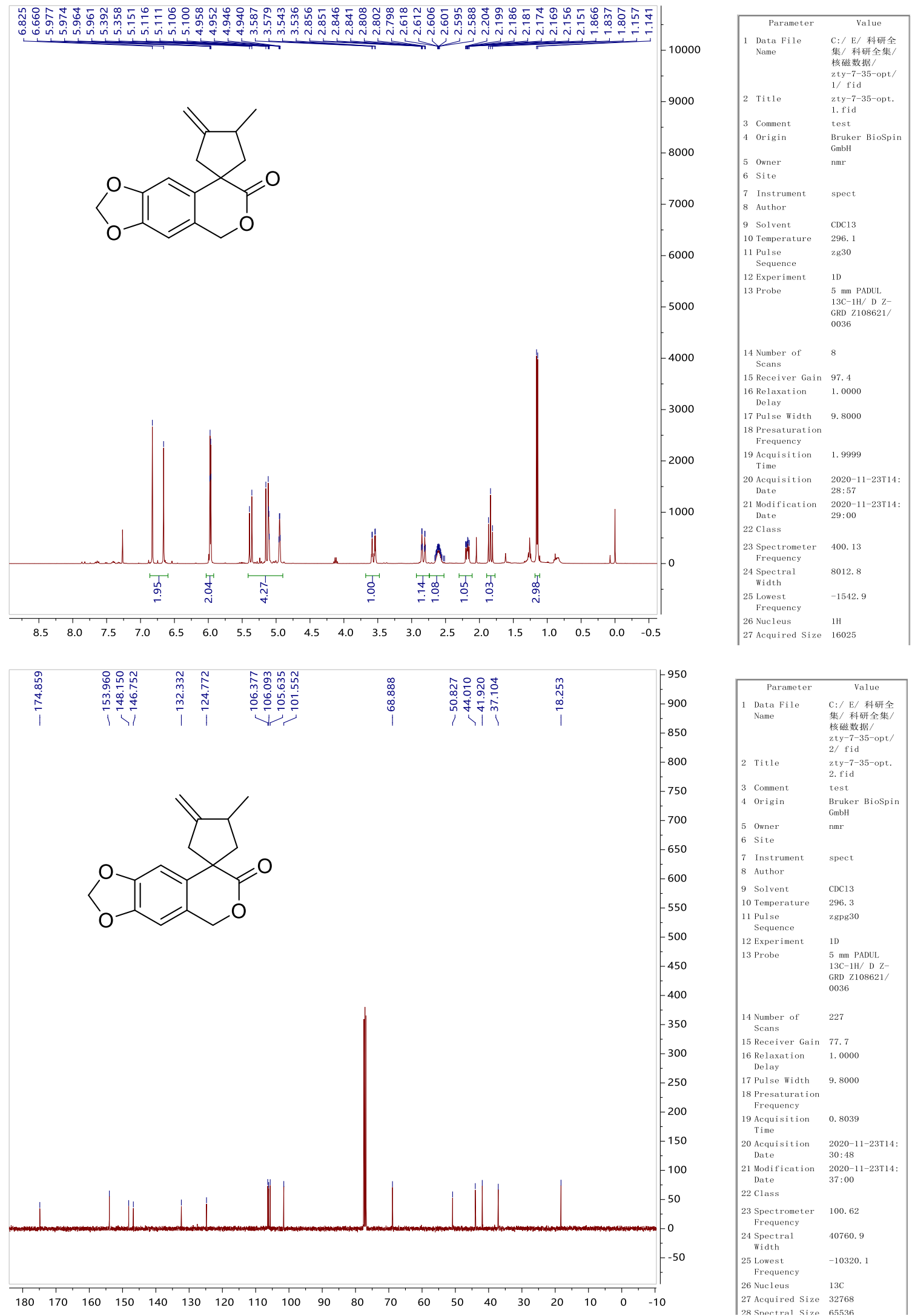


\section{2-allyl-2-(2-(hydroxymethyl)phenyl)pent-4-en-1-ol (2p)}
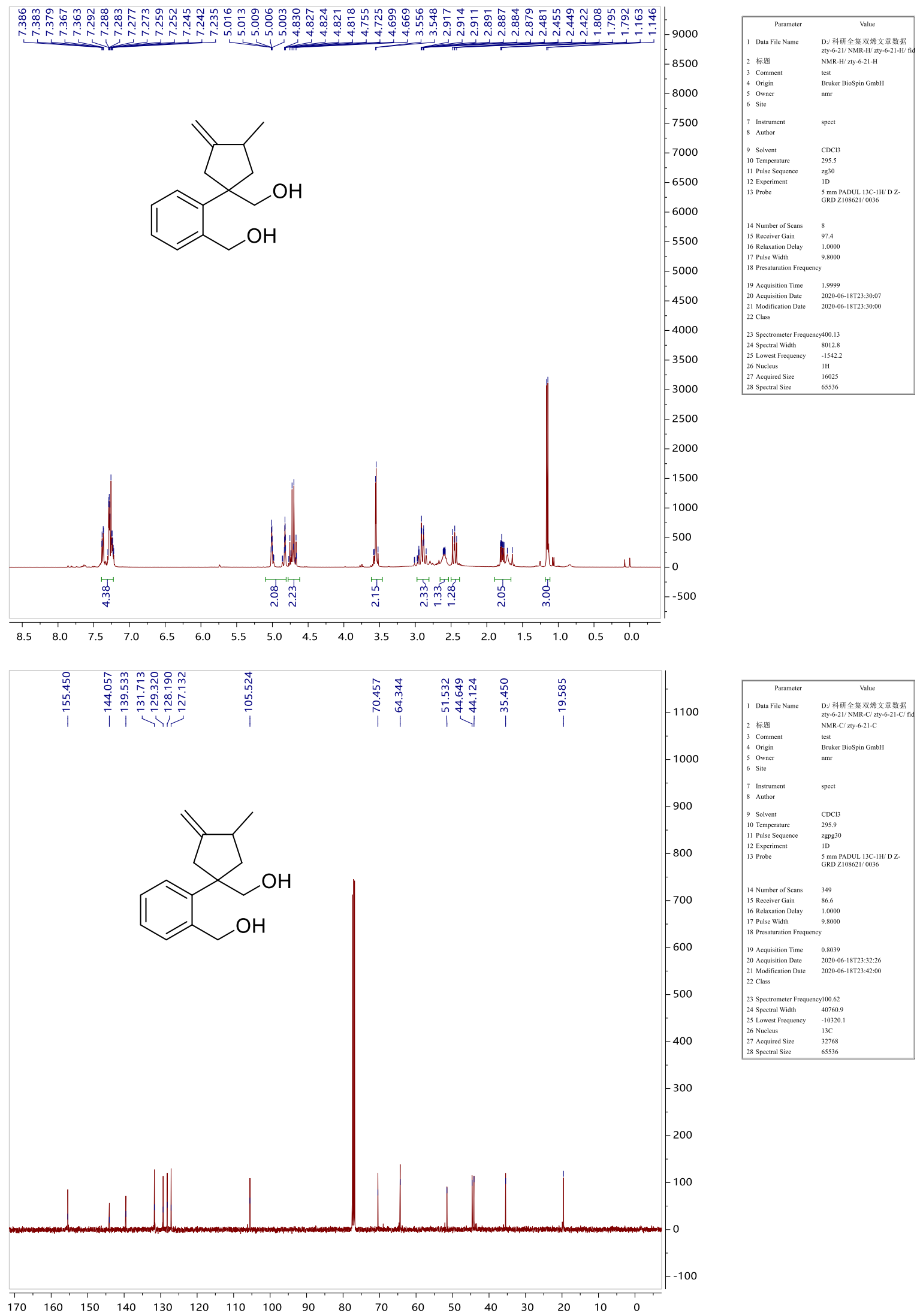
(2-(1-(hydroxymethyl)-3-methyl-4-methylenecyclopentyl)-5-methoxyphenyl)methanol (2q)
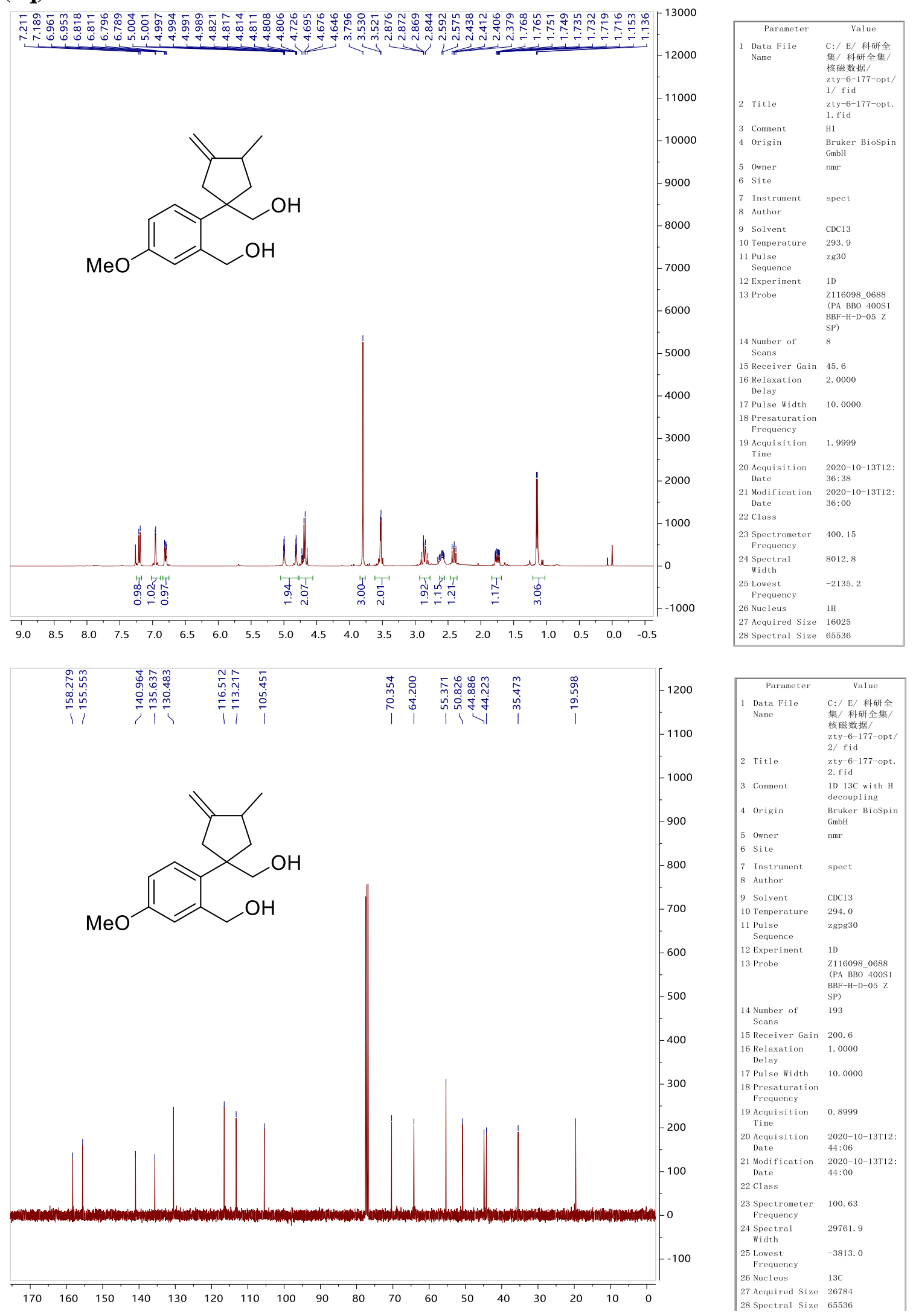

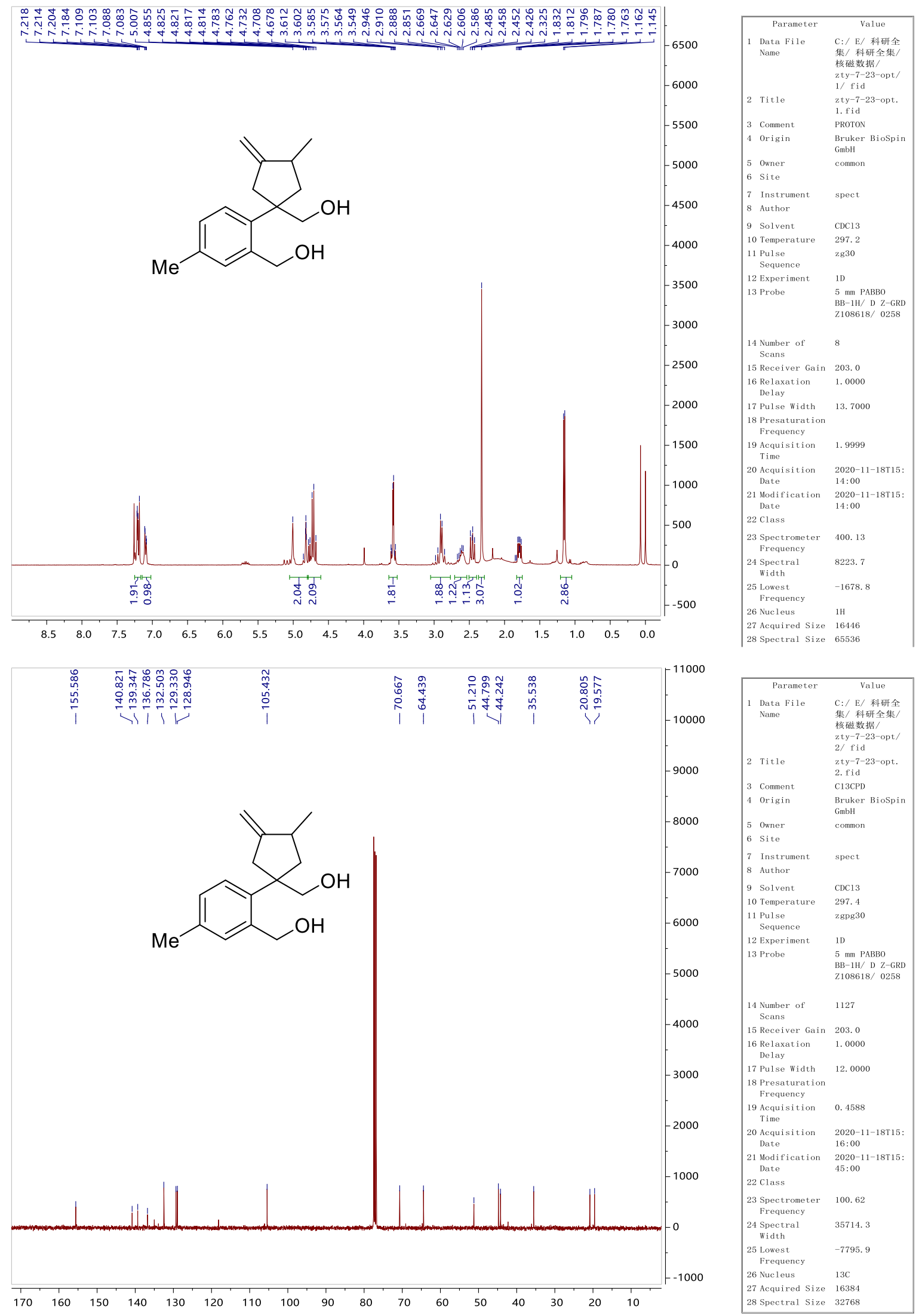
(1-(3-fluoro-2-(hydroxymethyl)phenyl)-3-methyl-4-methylenecyclopentyl)methanol (2s)
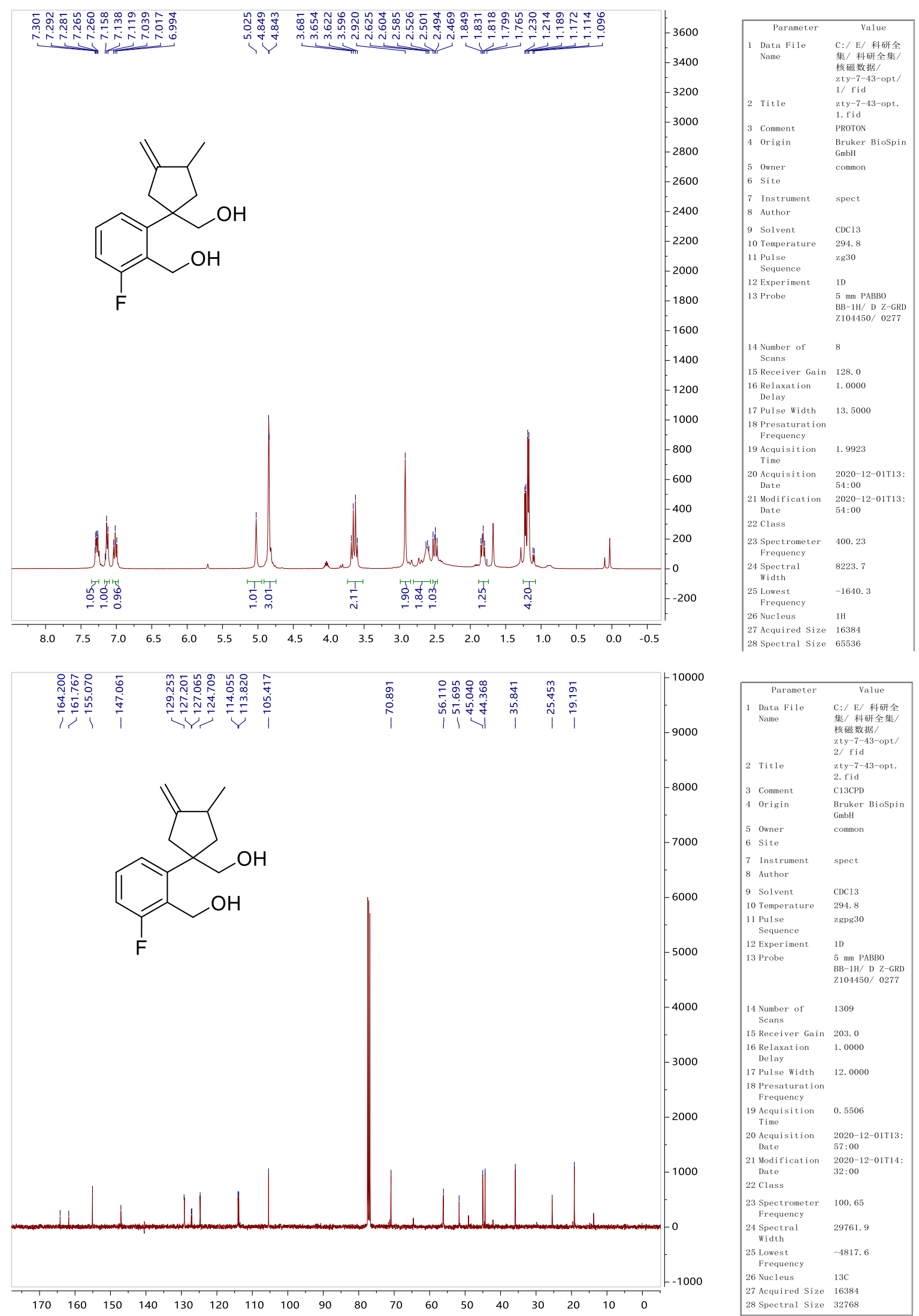

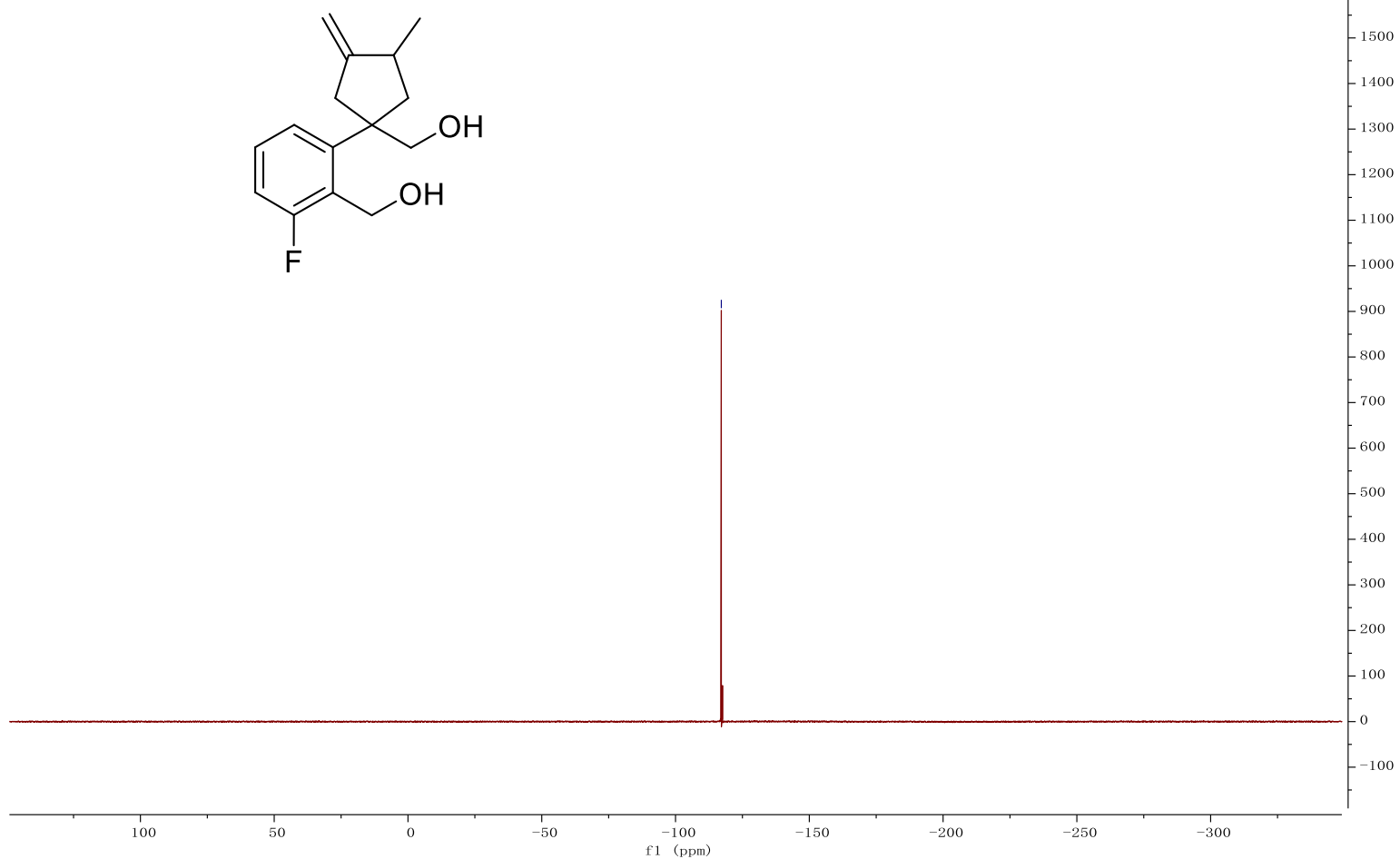
methyl 3-methyl-4-methylene-1-phenylcyclopentane-1-carboxylate (2t)

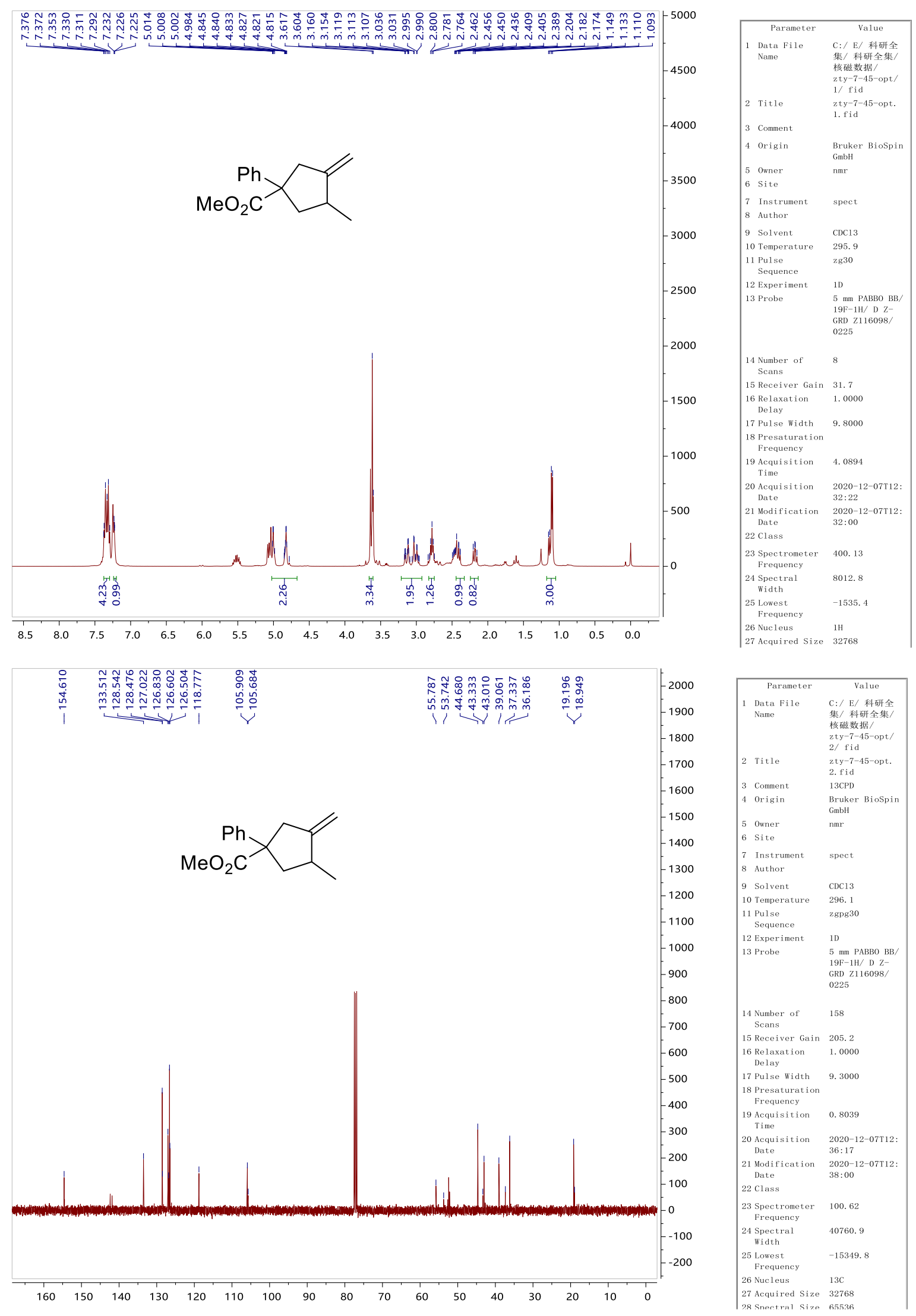



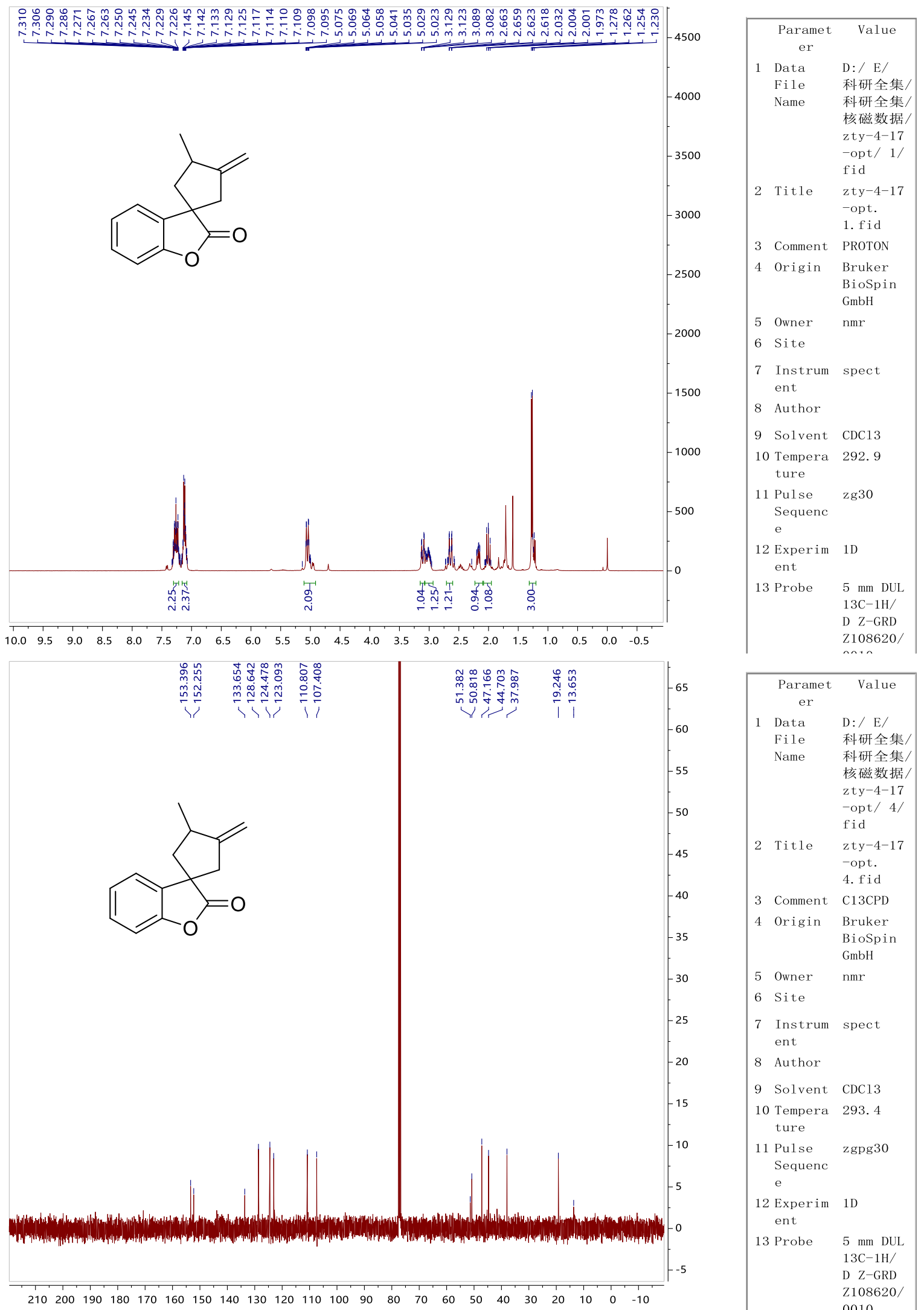

\begin{tabular}{|c|c|c|}
\hline & $\begin{array}{c}\text { Paramet } \\
\text { er }\end{array}$ & Value \\
\hline 1 & $\begin{array}{l}\text { Data } \\
\text { File } \\
\text { Name }\end{array}$ & $\begin{array}{l}\text { D:/ E/ } \\
\text { 科研全集/ } \\
\text { 科研全集/ } \\
\text { 核磁数据/ } \\
\text { zty-4-17 } \\
\text {-opt/ 4/ } \\
\text { fid }\end{array}$ \\
\hline 2 & Title & $\begin{array}{l}\text { zty-4-17 } \\
- \text { opt. } \\
4 . \text { fid }\end{array}$ \\
\hline 3 & Comment & C13CPD \\
\hline 4 & Origin & $\begin{array}{l}\text { Bruker } \\
\text { BioSpin } \\
\text { GmbH }\end{array}$ \\
\hline 5 & Owner & $\mathrm{nmr}$ \\
\hline 6 & Site & \\
\hline 7 & $\begin{array}{l}\text { Instrum } \\
\text { ent }\end{array}$ & spect \\
\hline 8 & Author & \\
\hline 9 & Solvent & $\mathrm{CDC} 13$ \\
\hline 10 & $\begin{array}{l}\text { Tempera } \\
\text { ture }\end{array}$ & 293.4 \\
\hline 11 & $\begin{array}{l}\text { Pulse } \\
\text { Sequenc } \\
\text { e }\end{array}$ & zgpg30 \\
\hline 12 & $\begin{array}{l}\text { Experim } \\
\text { ent }\end{array}$ & 1D \\
\hline 13 & Probe & $\begin{array}{l}5 \mathrm{~mm} \mathrm{DUL} \\
13 \mathrm{C}-1 \mathrm{H} / \\
\mathrm{D} \text { Z-GRD } \\
\text { Z108620/ } \\
0010\end{array}$ \\
\hline
\end{tabular}




\section{4,4-bis $((E)$-allyl-3- $d)$ isochroman-3-one (1a- $\left.d^{I}\right)$}
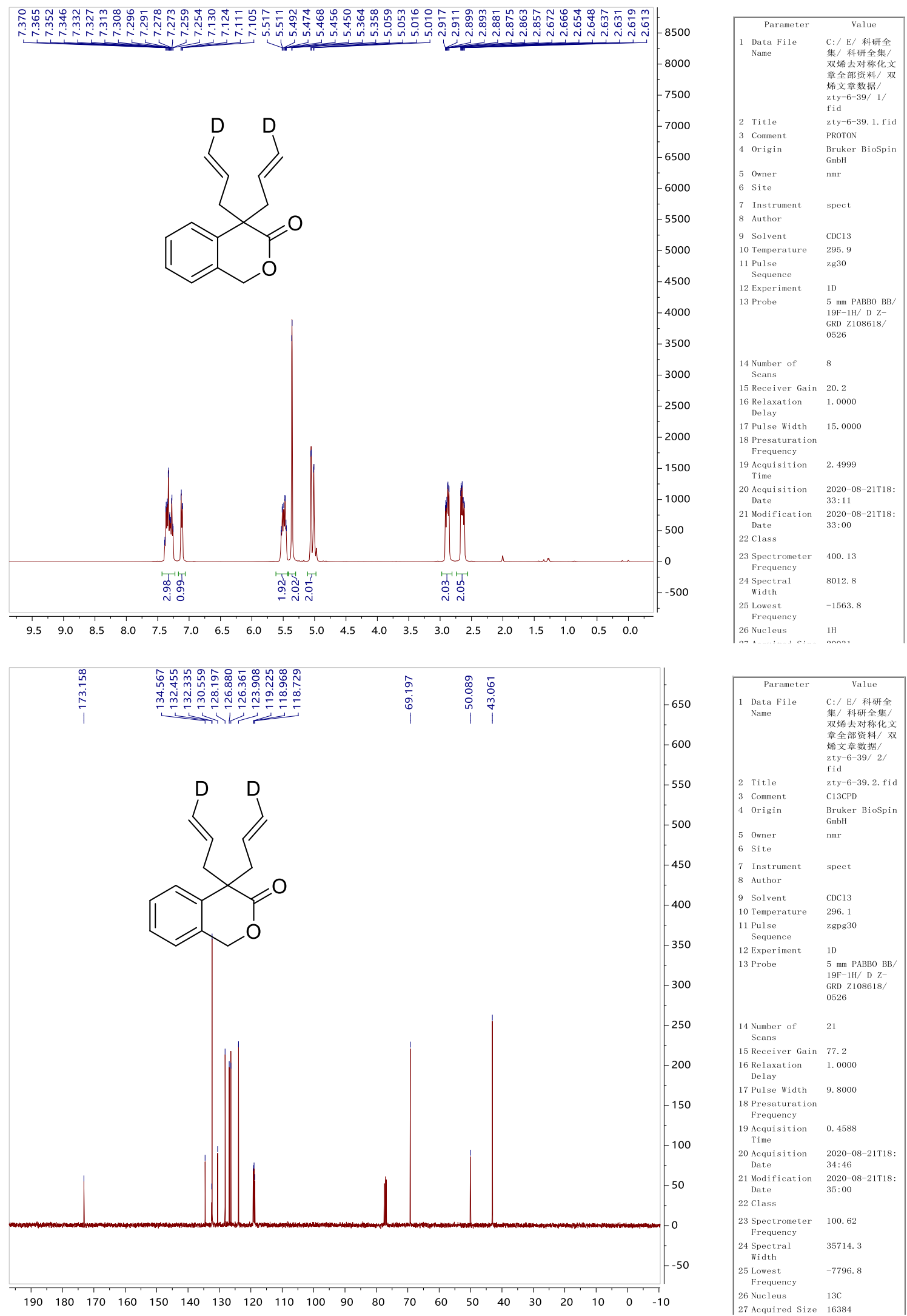


\section{4,4-bis(allyl-2-d)isochroman-3-one (1a- $\left.d^{2}\right)$}
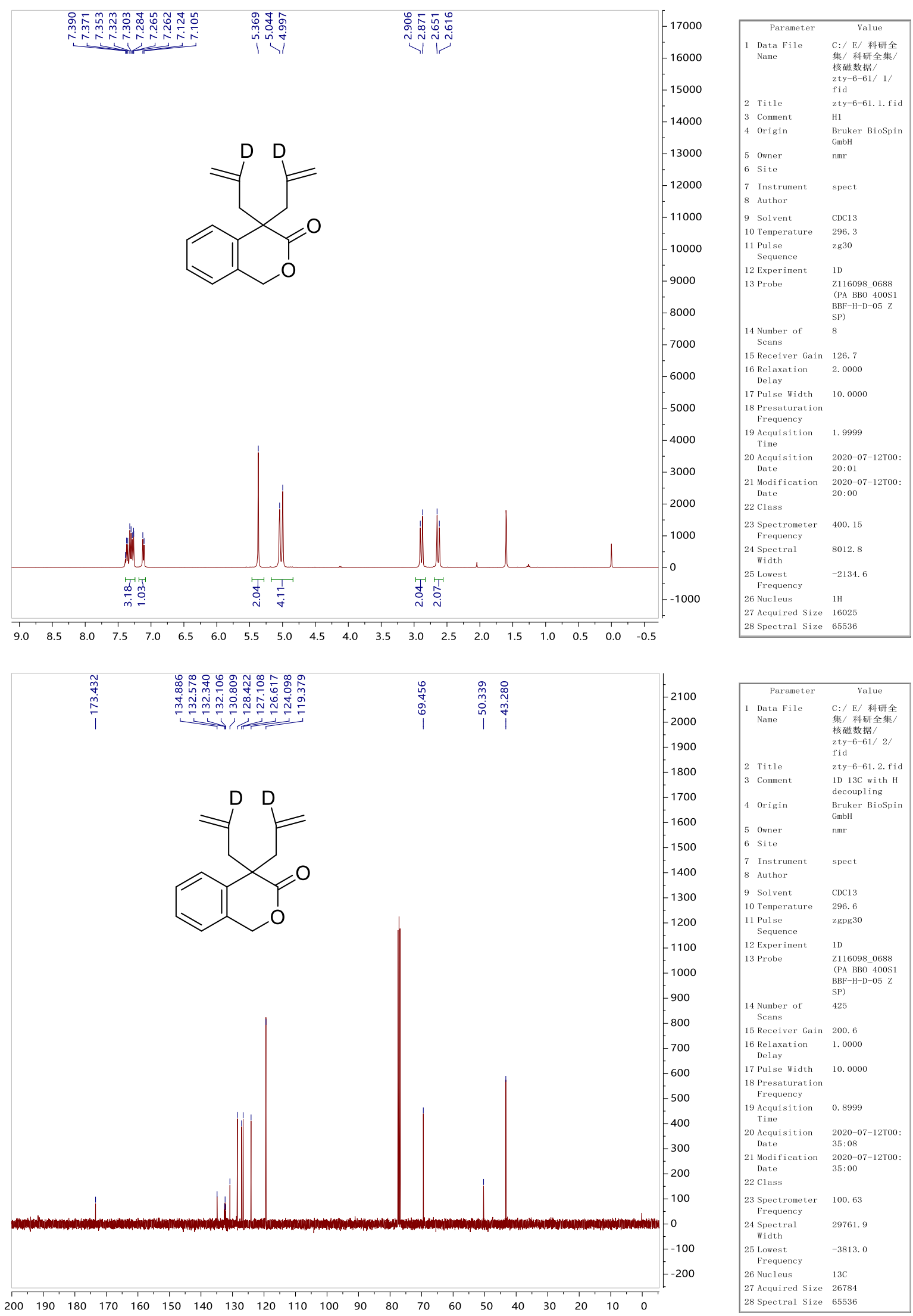
3-(methyl- $d$ )-4-(methylene- $\left.d_{2}\right)$ spiro[cyclopentane-1,4'-isochroman]-3'-one (2a-d $\left.{ }^{1}\right)$
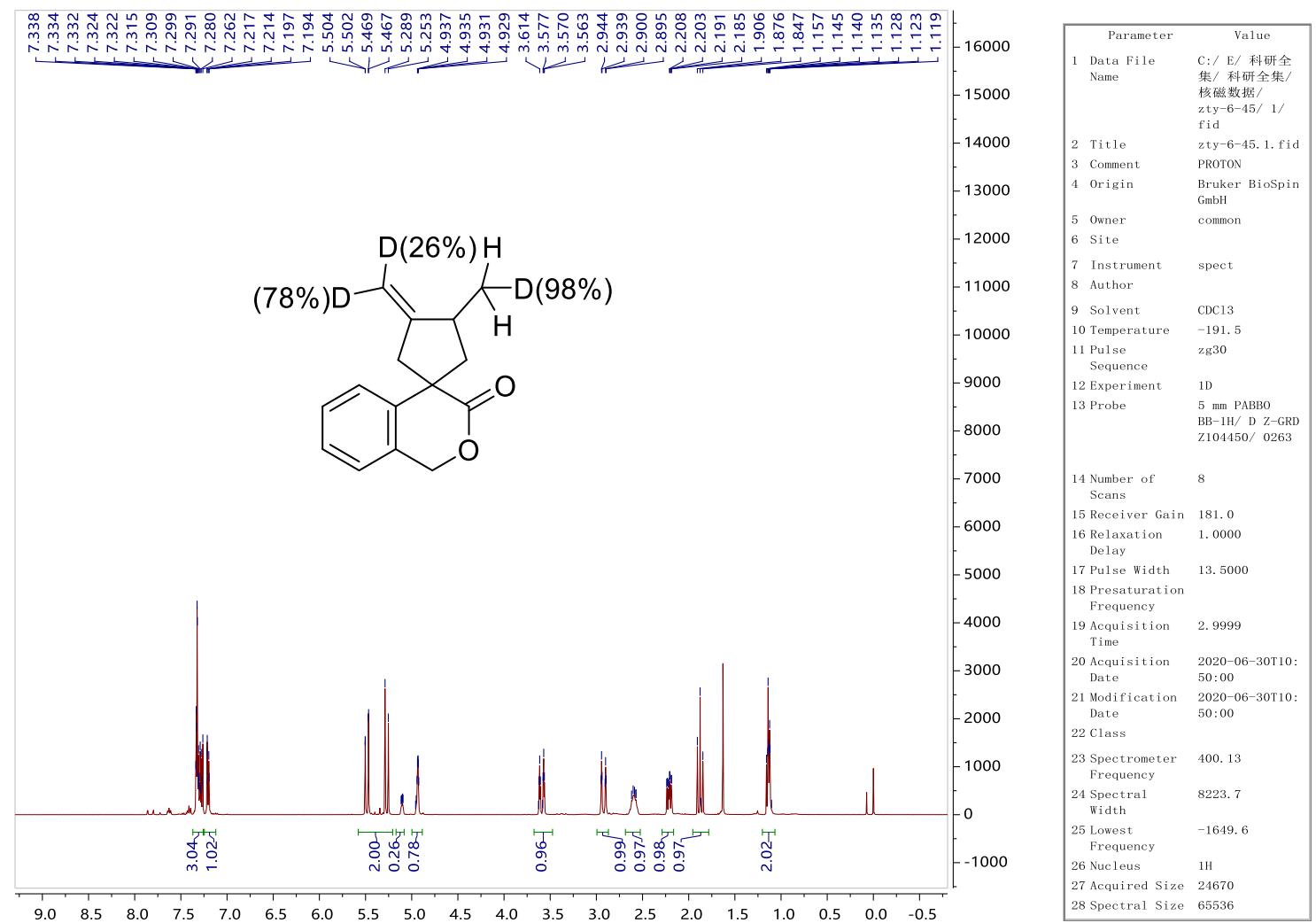

3-(methyl- $d$ )-4-methylenespiro[cyclopentane-1,4'-isochroman]-3'-one-3- $d\left(2 \mathrm{a}-d^{2}\right)$

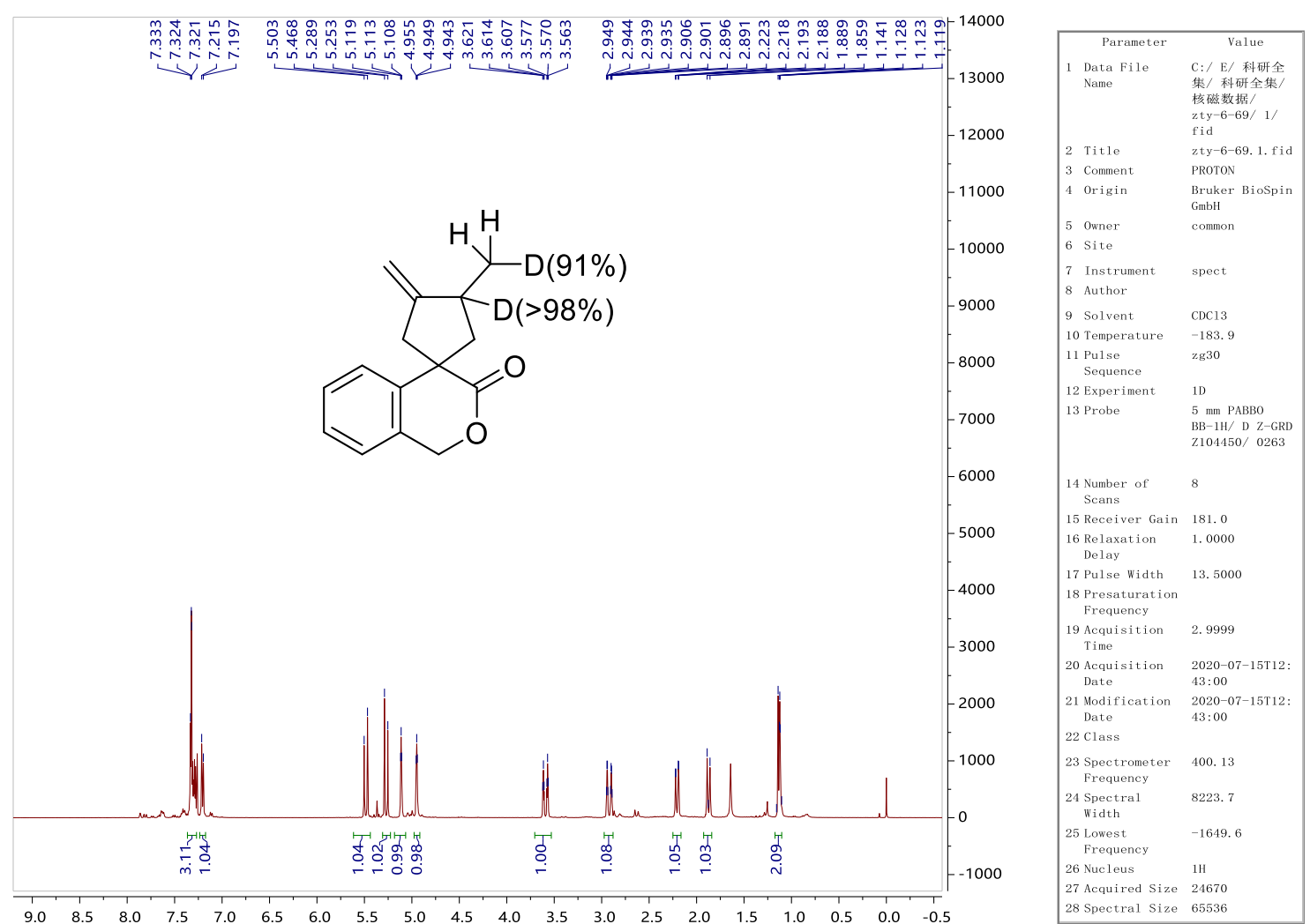




\section{HPLC Charts of Products}

3-methyl-4-methylenespiro[cyclopentane-1,4'-isochroman]-3'-one (2a)
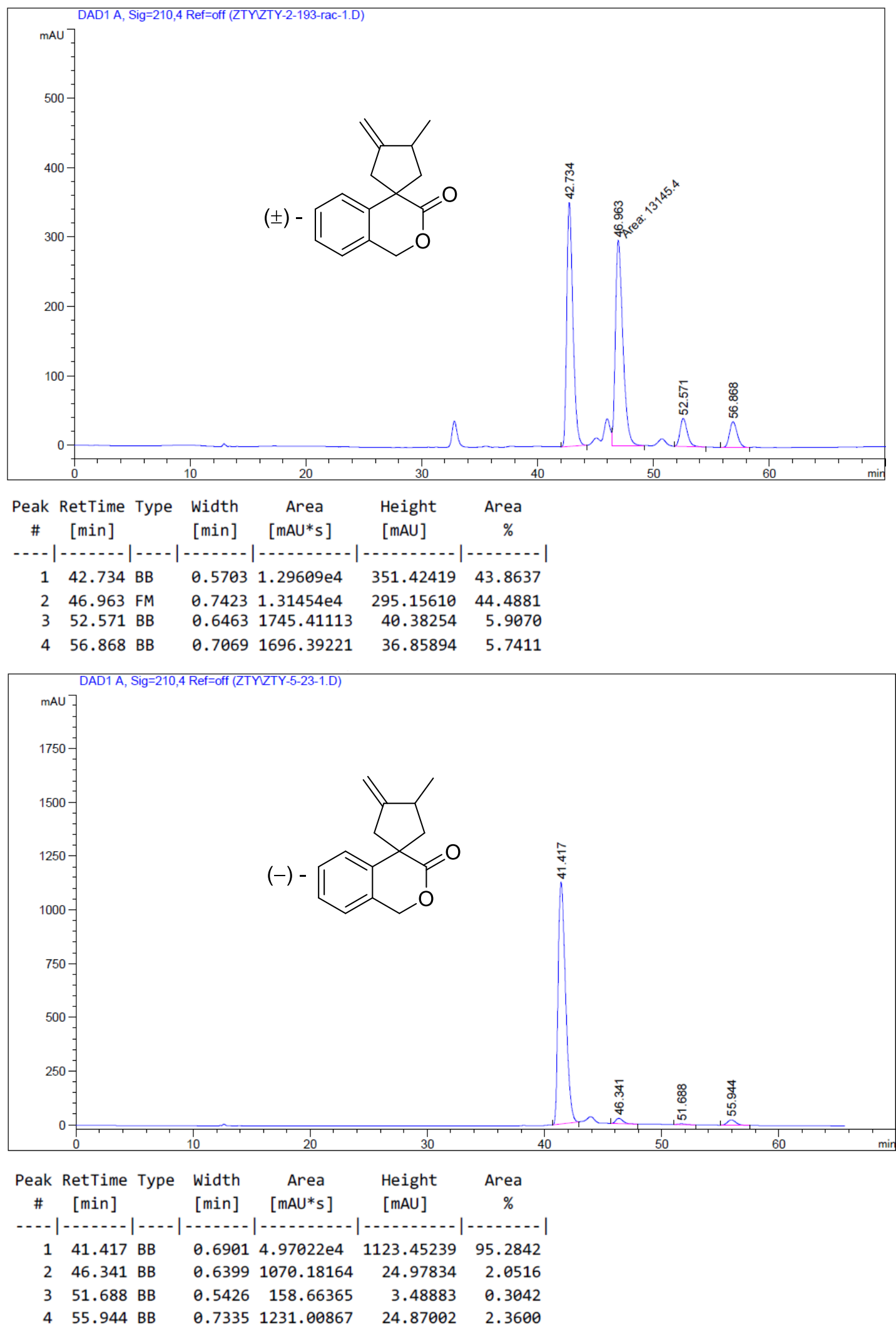
3,7'-dimethyl-4-methylenespiro[cyclopentane-1,4'-isochroman]-3'-one (2b)
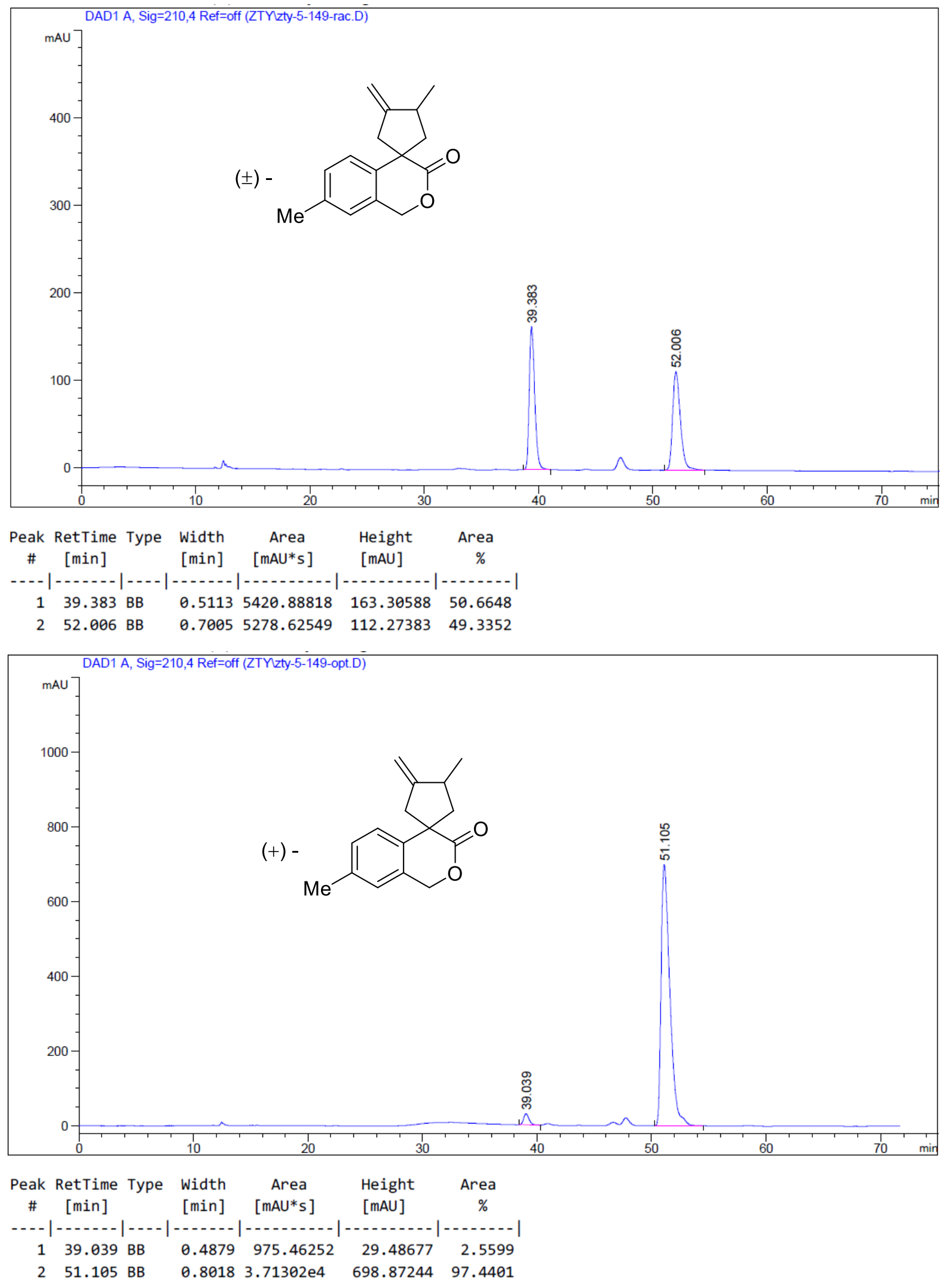
7'-chloro-3-methyl-4-methylenespiro[cyclopentane-1,4'-isochroman]-3'-one (2c)

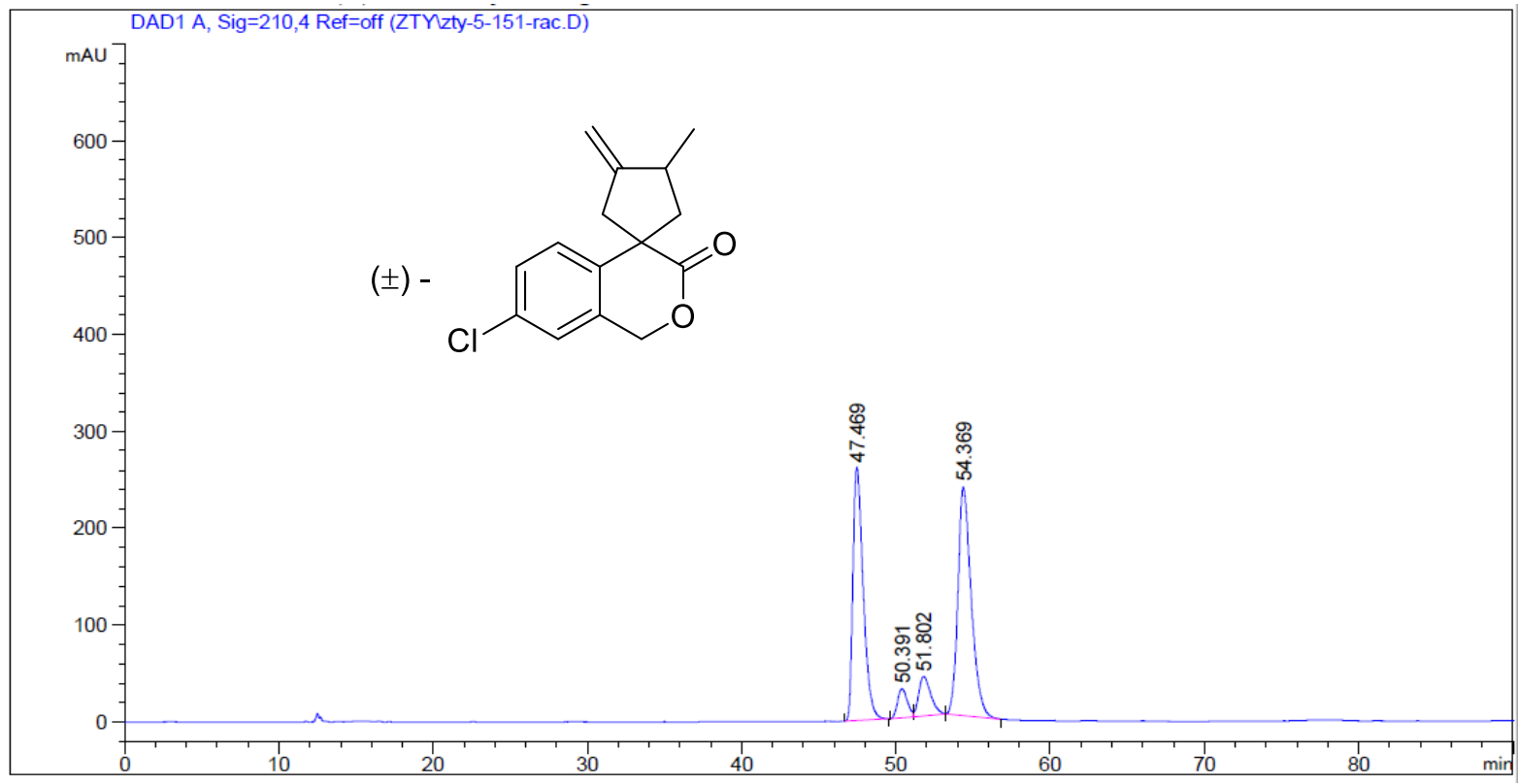

\begin{tabular}{|c|c|c|c|c|c|c|}
\hline $\begin{array}{c}\text { Peak } \\
\quad \#\end{array}$ & $\begin{array}{c}\text { RetTime } \\
\text { [min] }\end{array}$ & Type & $\begin{array}{l}\text { Width } \\
\text { [min] }\end{array}$ & $\begin{array}{c}\text { Area } \\
{\left[\mathrm{mAU} U^{*} \mathrm{~s}\right]}\end{array}$ & $\begin{array}{l}\text { Height } \\
\text { [mAU] }\end{array}$ & $\begin{array}{c}\text { Area } \\
\%\end{array}$ \\
\hline & & & & & & 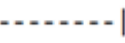 \\
\hline 1 & 47.469 & BB & 0.6981 & $1.20996 \mathrm{e} 4$ & 261.36295 & 40.6113 \\
\hline 2 & 50.391 & BV & 0.6464 & 1324.08386 & 30.26690 & 4.4442 \\
\hline 3 & 51.802 & VB & 0.7765 & 2212.54907 & 40.68368 & 7.4262 \\
\hline 4 & 54.369 & BB & 0.8810 & $1.41574 \mathrm{e} 4$ & 236.28203 & 47.5183 \\
\hline
\end{tabular}

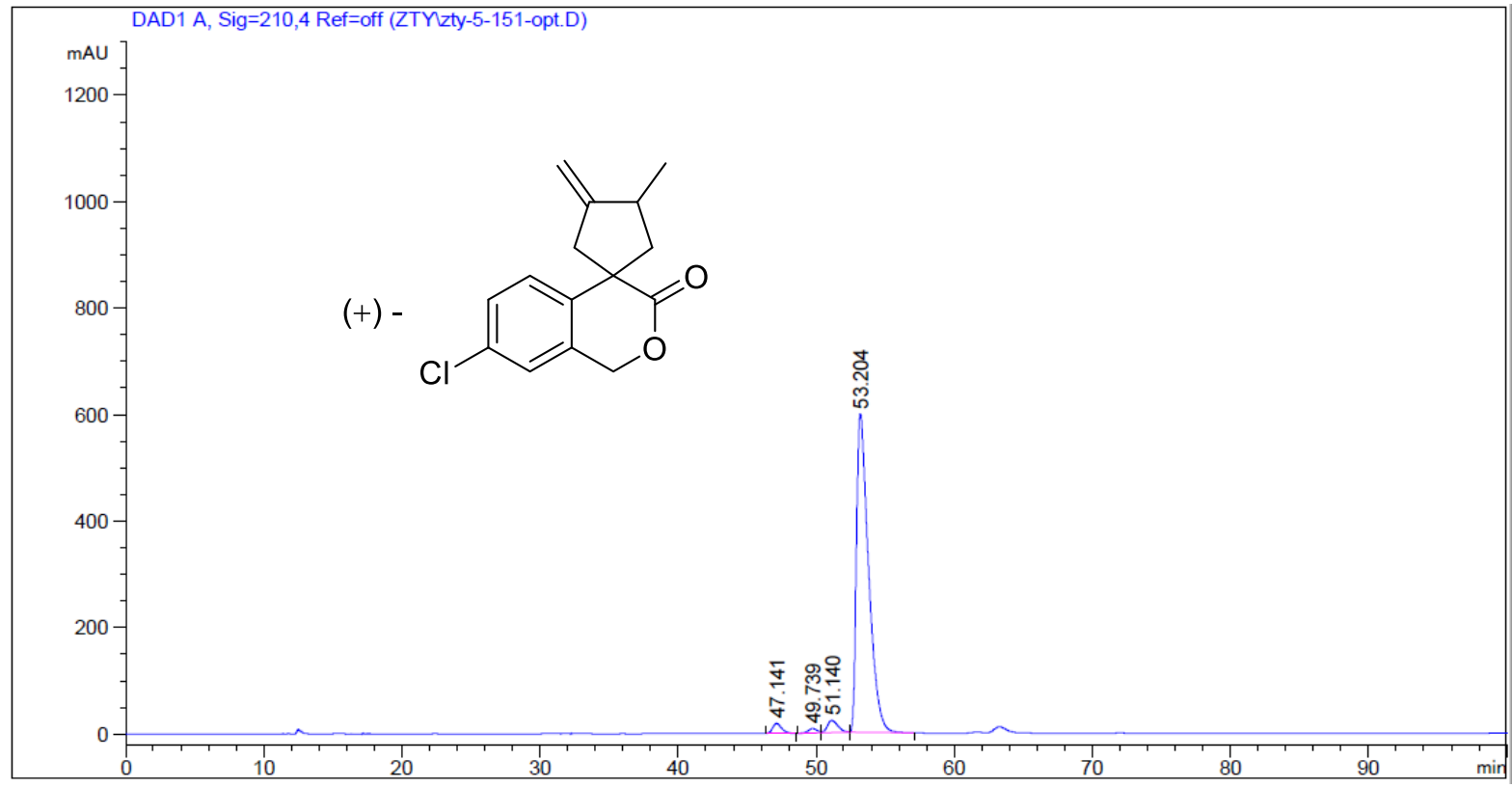

\begin{tabular}{cccccc}
$\begin{array}{c}\text { Peak RetTime Type } \\
\text { \# } \\
\text { [min] }\end{array}$ & $\begin{array}{c}\text { Width } \\
\text { [min] }\end{array}$ & $\begin{array}{c}\text { Area } \\
\text { [mAU*s }\end{array}$ & \multicolumn{1}{c}{$\begin{array}{c}\text { Height } \\
\text { [mAU] }\end{array}$} & \multicolumn{1}{c}{$\begin{array}{c}\text { Area } \\
\%\end{array}$} \\
\hline 1 & 47.141 BB & 0.6084 & 862.51904 & 18.89975 & 2.2154 \\
2 & 49.739 BV & 0.5566 & 406.74643 & 8.71333 & 1.0448 \\
3 & 51.140 VB & 0.6887 & 1213.35168 & 23.10571 & 3.1166 \\
4 & 53.204 BB & 0.8908 & $3.64497 e 4$ & 598.28705 & 93.6233
\end{tabular}


7'-methoxy-3-methyl-4-methylenespiro[cyclopentane-1,4'-isochroman]-3'-one (2d)
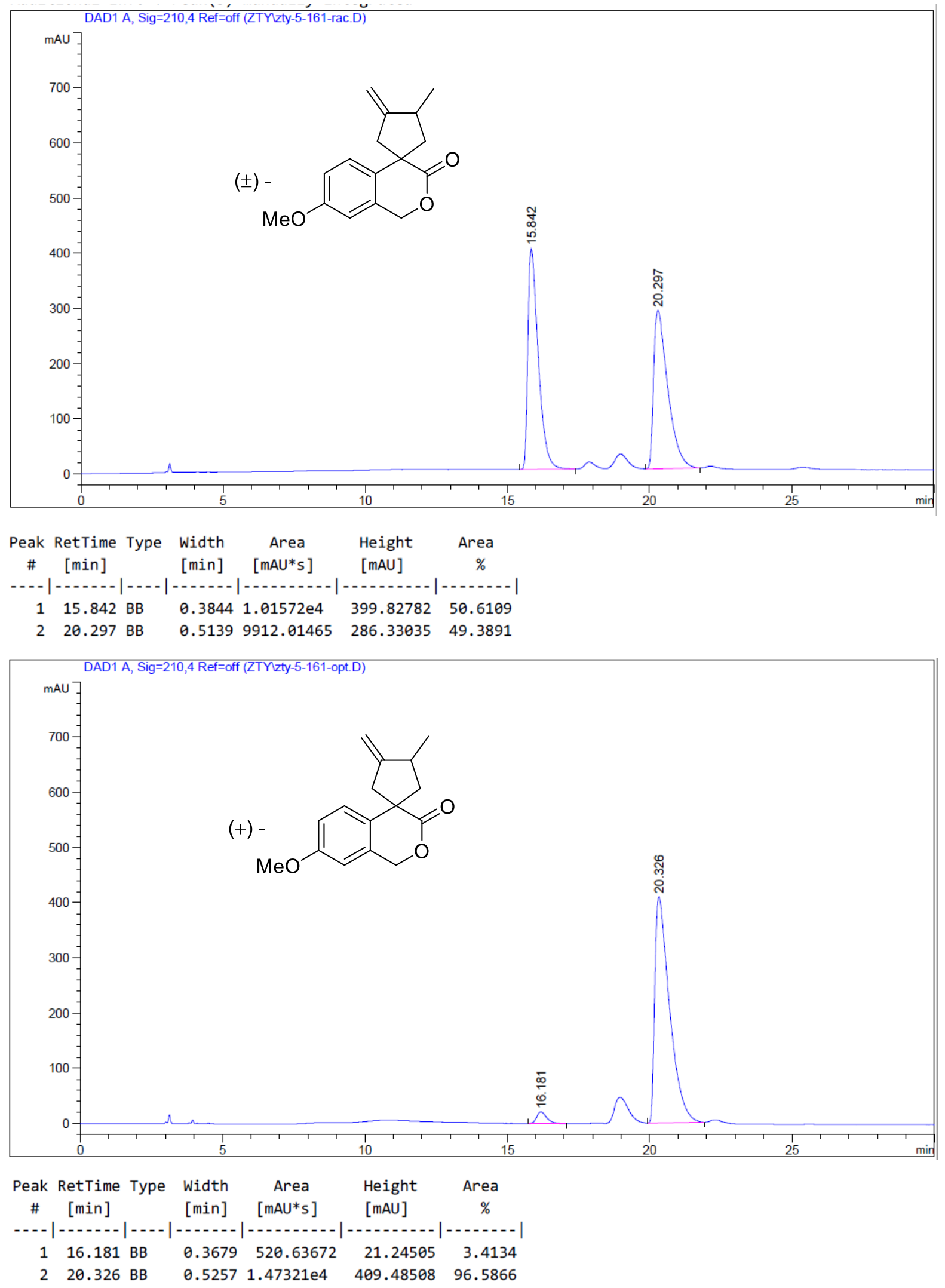
3,6'-dimethyl-4-methylenespiro[cyclopentane-1,4'-isochroman]-3'-one (2e)
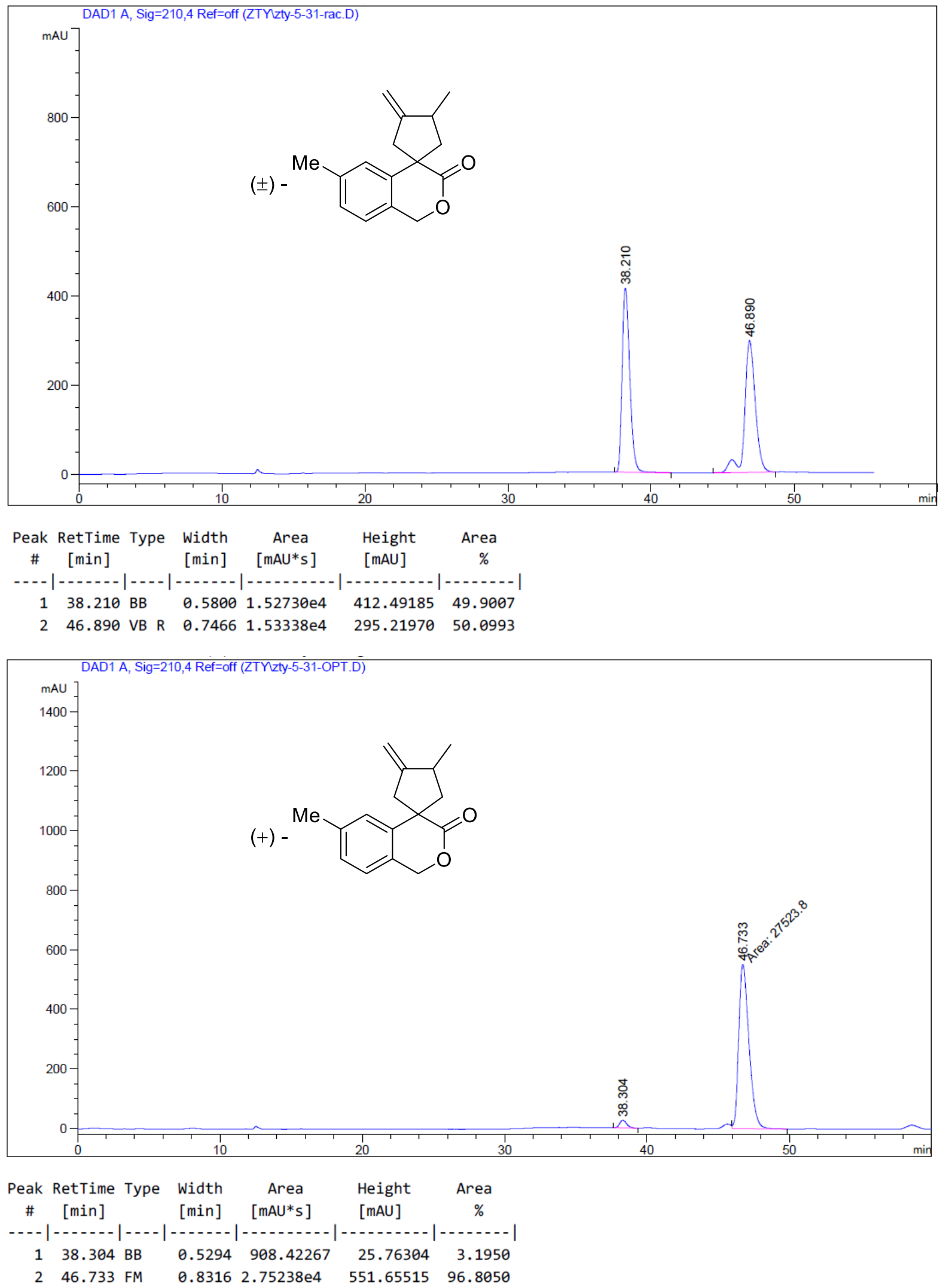
6'-chloro-3-methyl-4-methylenespiro[cyclopentane-1,4'-isochroman]-3'-one (2f)

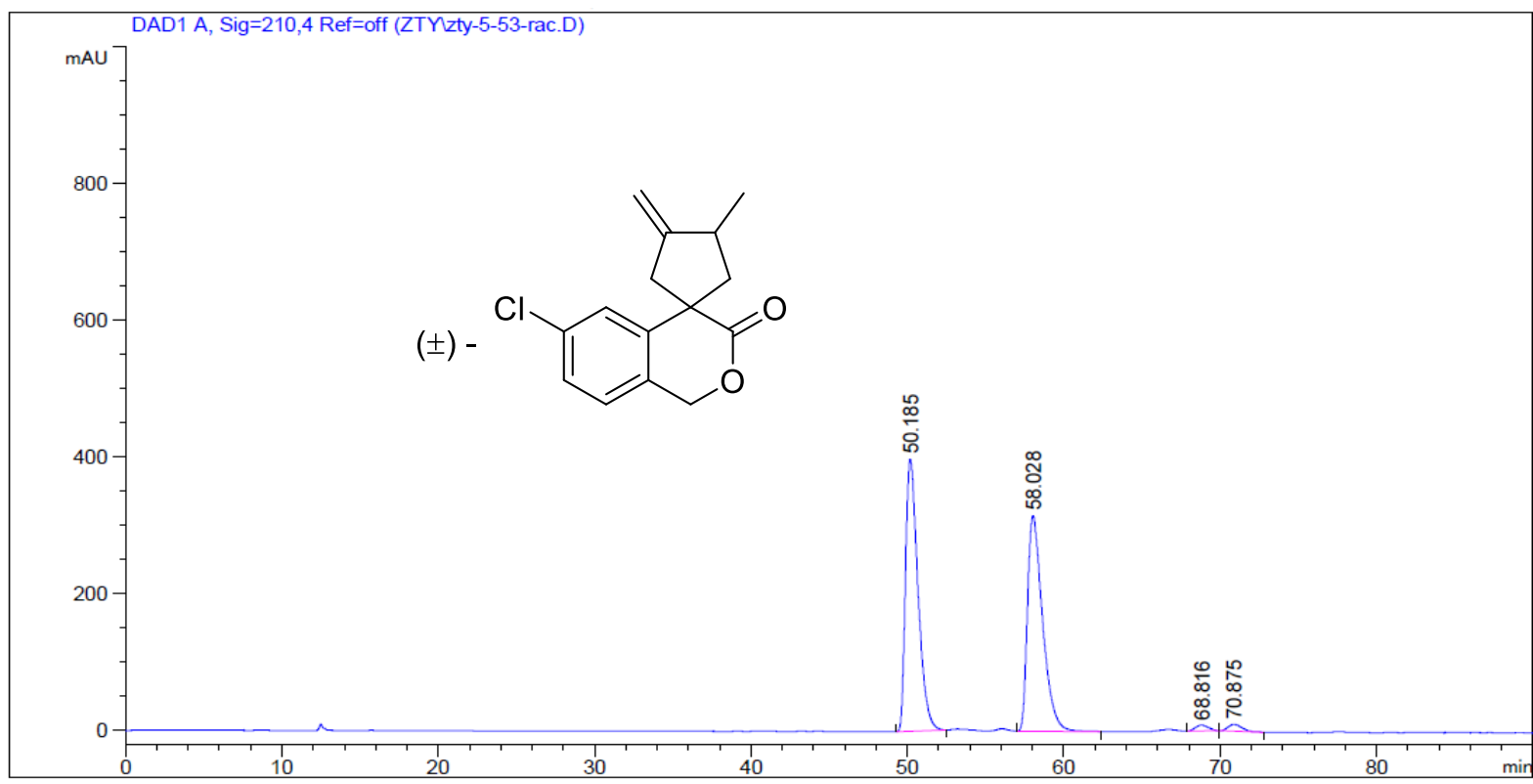

\begin{tabular}{|c|c|c|c|c|c|c|}
\hline $\begin{array}{c}\text { Peak } \\
\quad \#\end{array}$ & $\begin{array}{c}\text { RetTime } \\
\text { [min] }\end{array}$ & Type & $\begin{array}{l}\text { Width } \\
\text { [min] }\end{array}$ & $\begin{array}{c}\text { Area } \\
{\left[\mathrm{mAU}^{*} \mathrm{~s}\right]}\end{array}$ & $\begin{array}{l}\text { Height } \\
{[\mathrm{mAU}]}\end{array}$ & $\begin{array}{c}\text { Area } \\
\%\end{array}$ \\
\hline & & & & $-\cdots$ & . & $---\cdot-\cdot-1$ \\
\hline 1 & 50.185 & BB & 0.8360 & $2.20517 \mathrm{e} 4$ & 398.22241 & 49.6591 \\
\hline 2 & 58.028 & BB & 0.9919 & $2.12250 \mathrm{e} 4$ & 315.10065 & 47.7976 \\
\hline 3 & 68.816 & BB & 0.6958 & 511.57742 & 8.65246 & 1.1520 \\
\hline 4 & 70.875 & BB & 0.7410 & 617.82245 & 9.91448 & 1.3913 \\
\hline
\end{tabular}

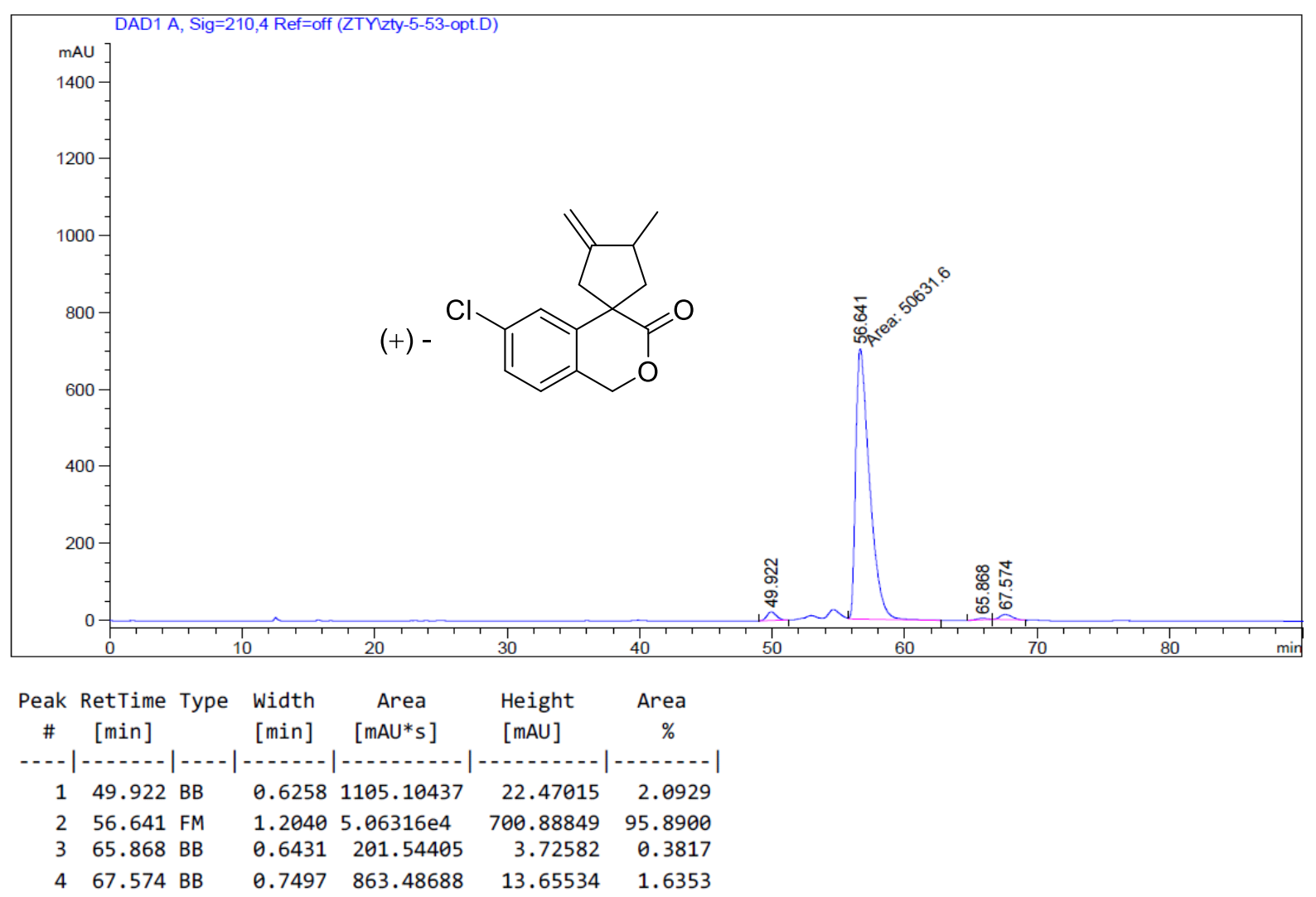


6'-methoxy-3-methyl-4-methylenespiro[cyclopentane-1,4'-isochroman]-3'-one (2g)
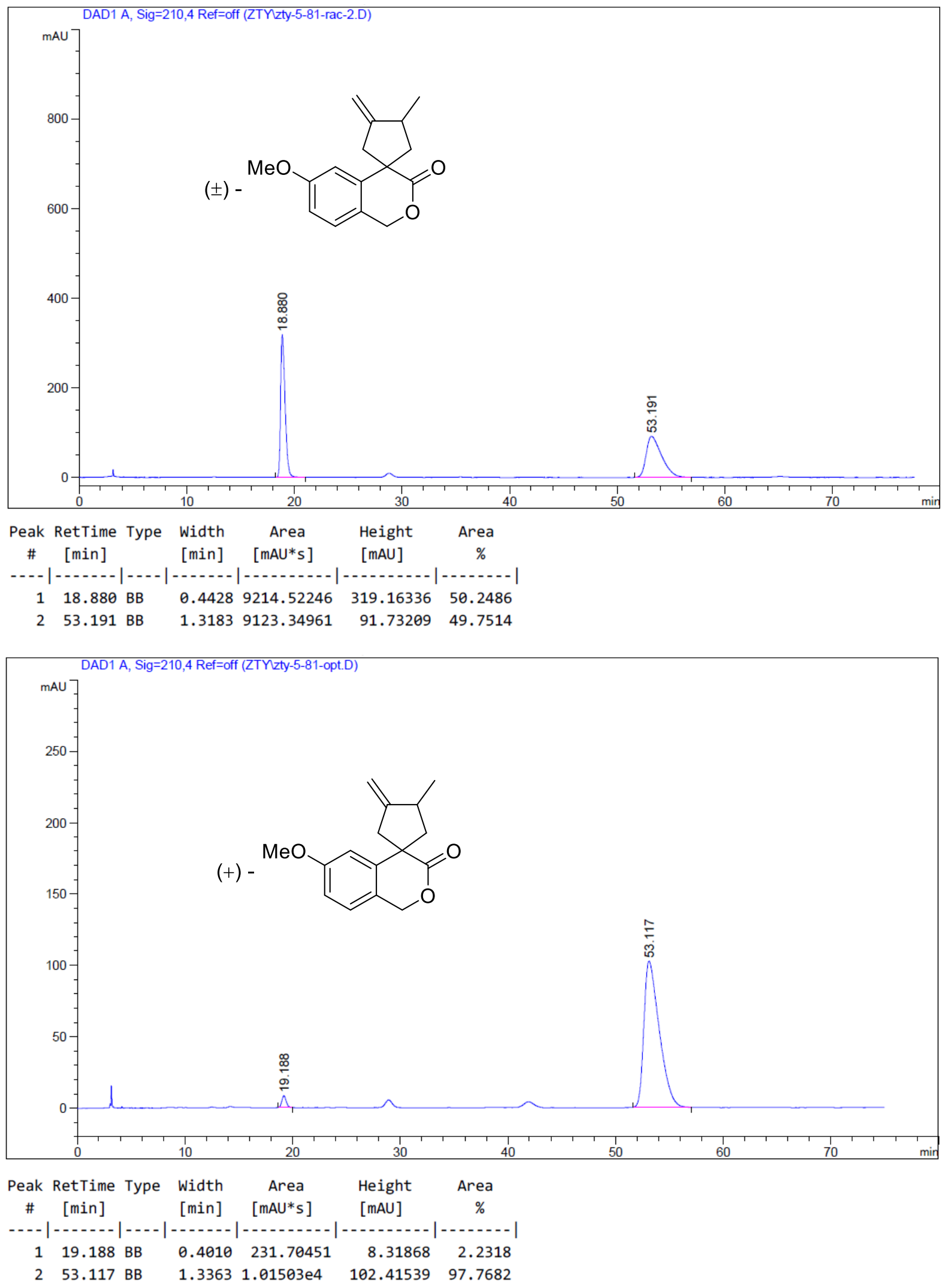
6'-bromo-3-methyl-4-methylenespiro[cyclopentane-1,4'-isochroman]-3'-one (2h)
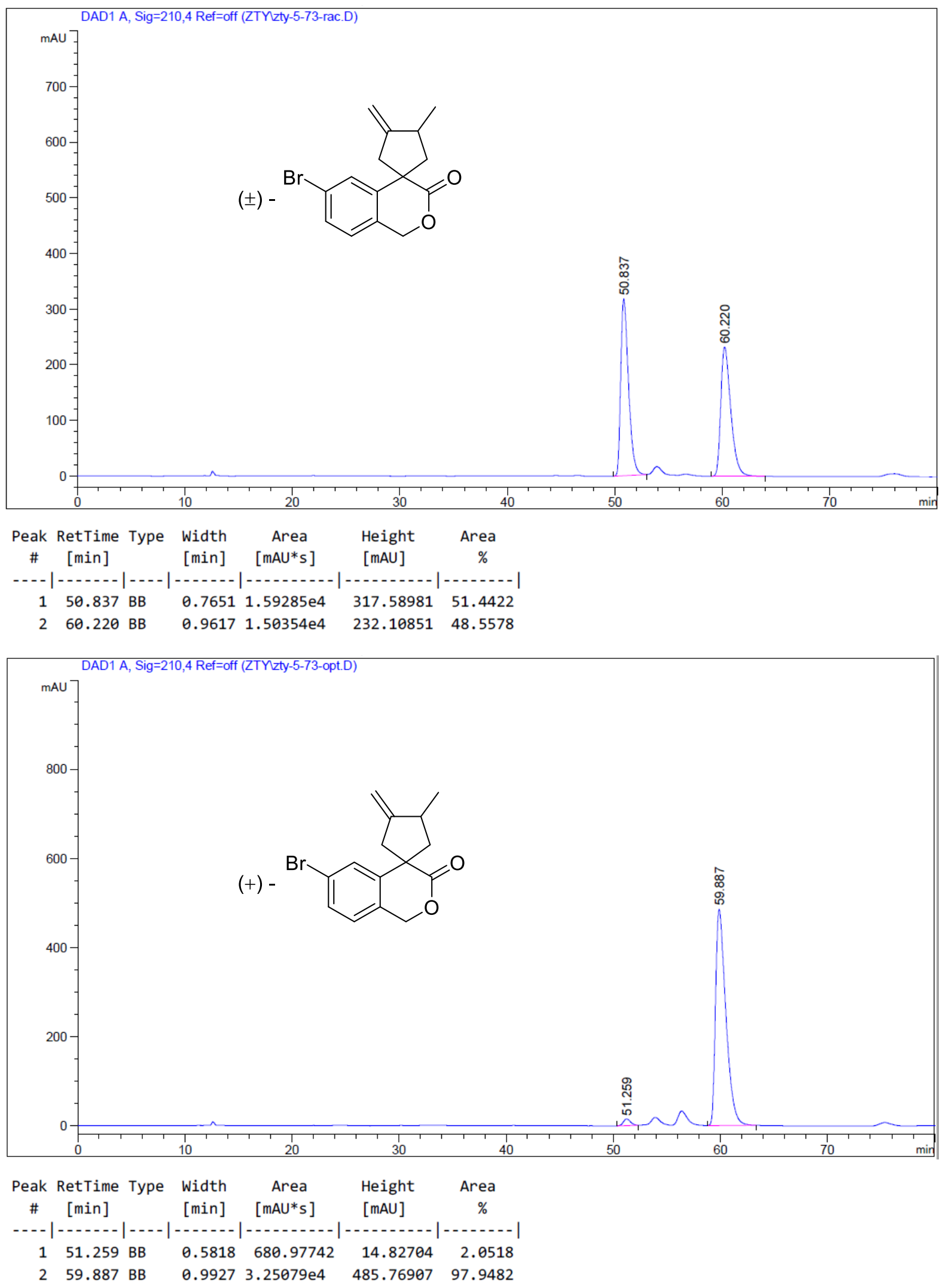
6'-fluoro-3-methyl-4-methylenespiro[cyclopentane-1,4'-isochroman]-3'-one (2i)
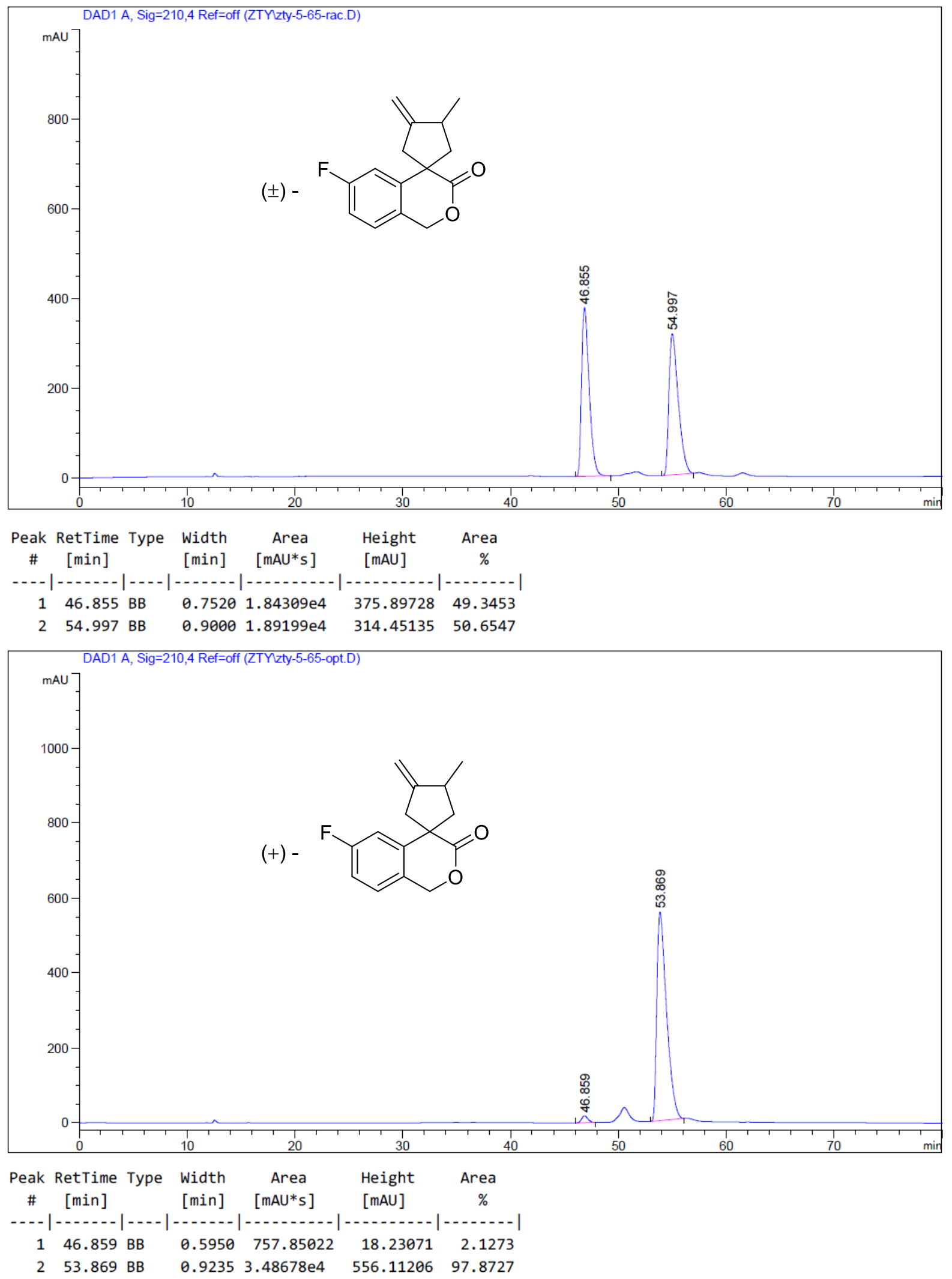
3-methyl-4-methylene-6' -(trifluoromethyl)spiro[cyclopentane-1,4'-isochroman]-3'-one (2g)
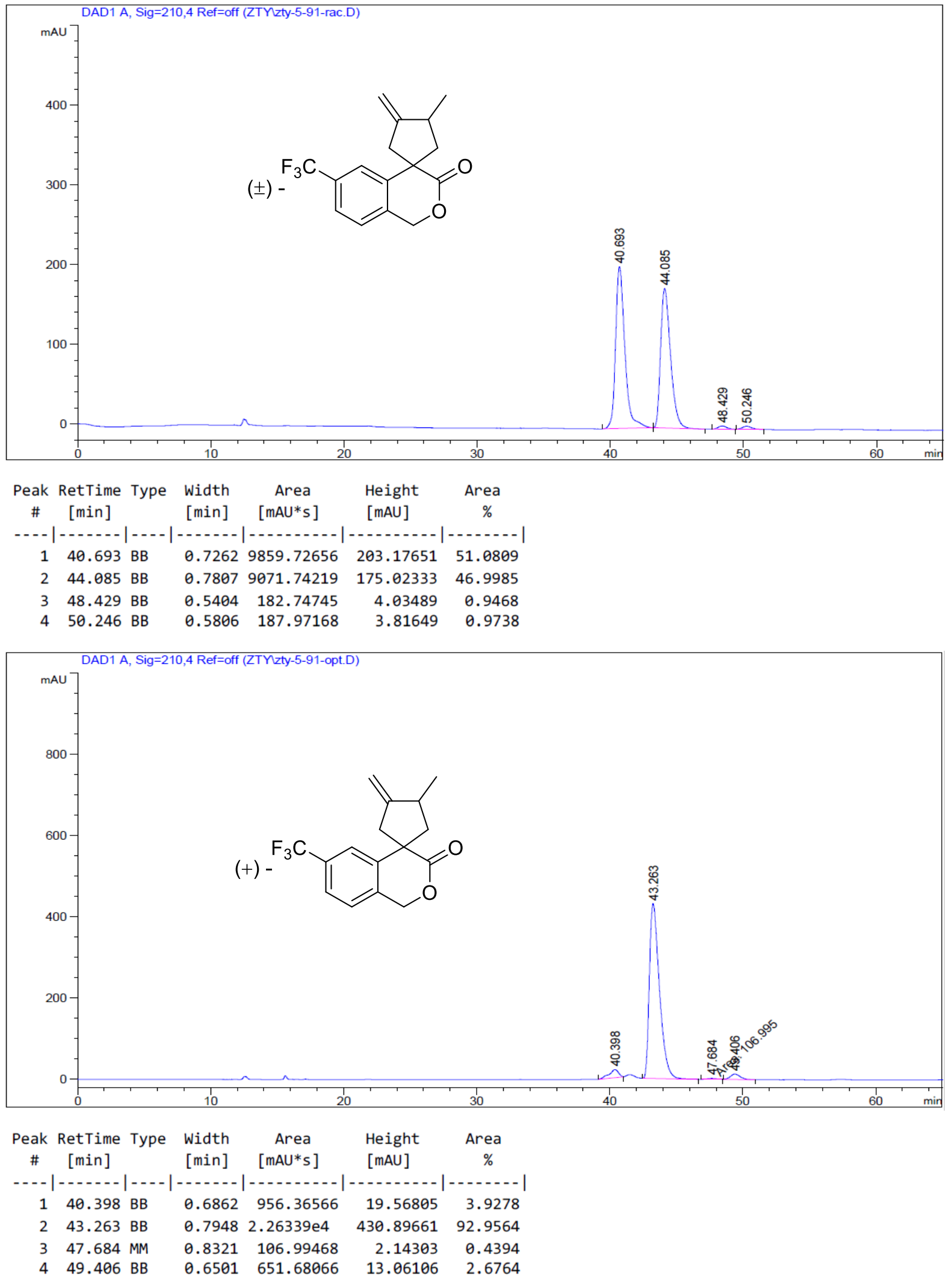
3-methyl-4-methylene-6' -(4-nitrophenyl)spiro[cyclopentane-1,4' -isochroman]-3'-one (2k)
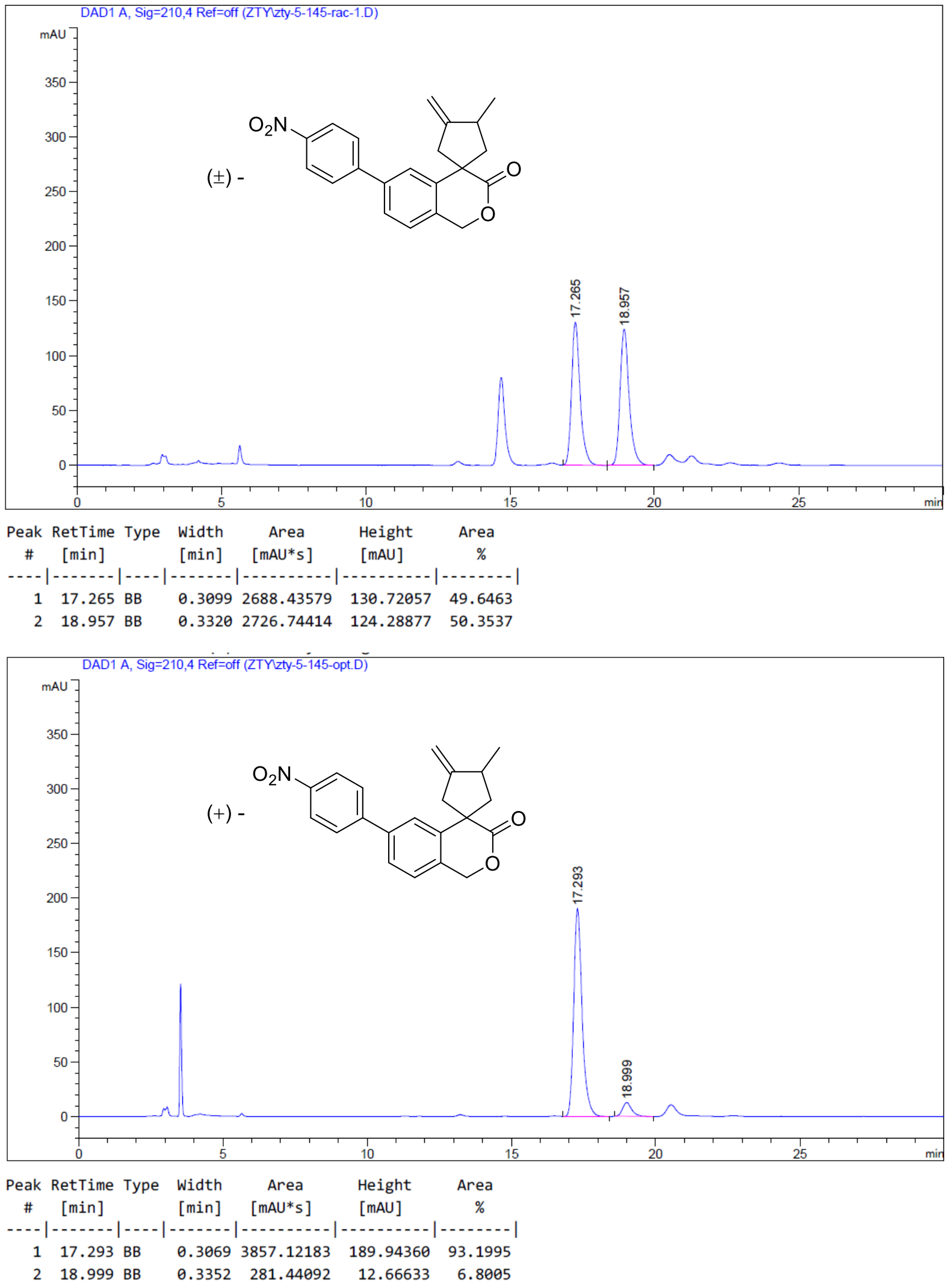
methyl 4-(3-methyl-4-methylene-3'-oxospiro[cyclopentane-1,4'-isochroman]-6'-

yl)benzoate (2l)
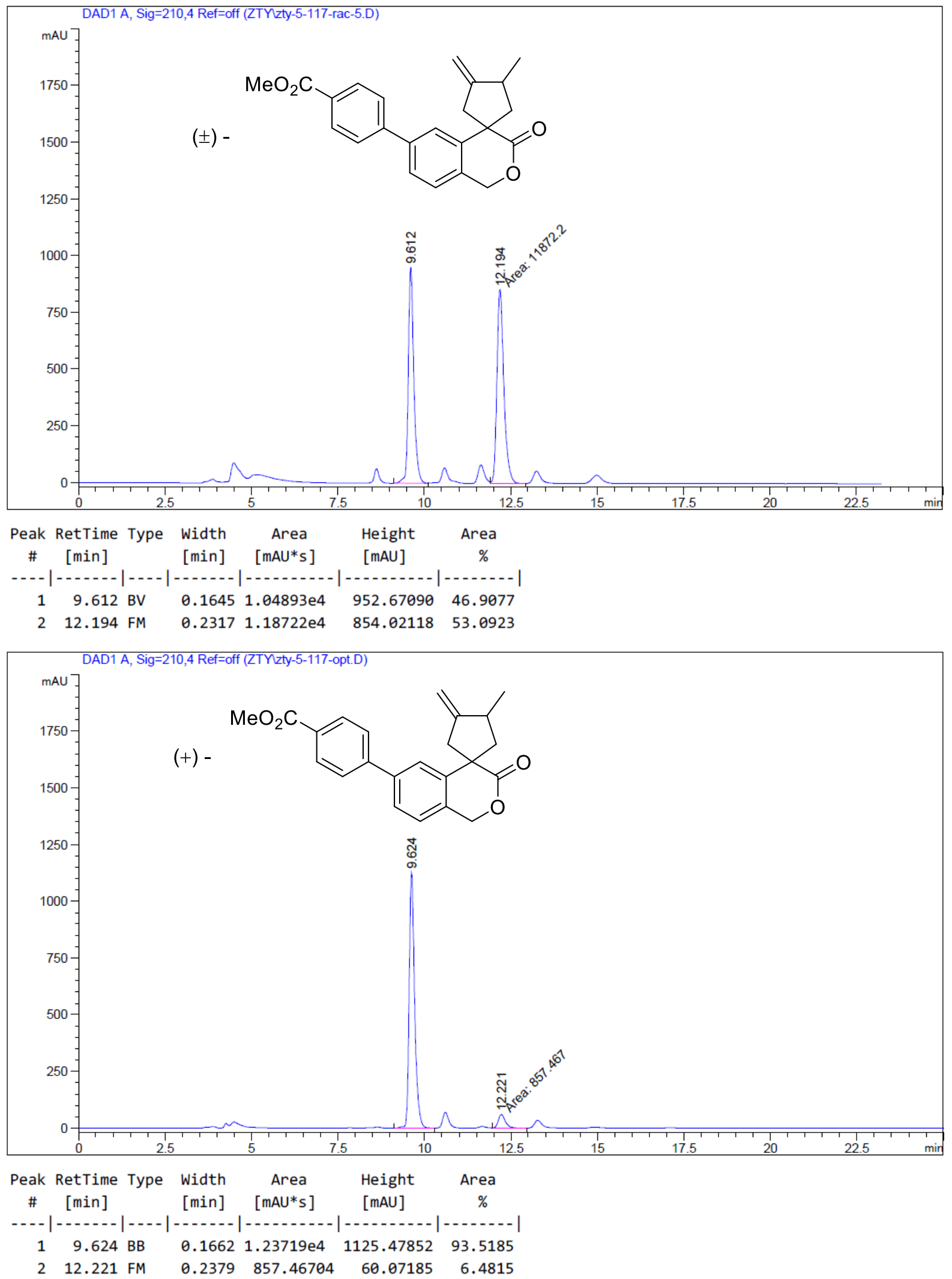
8'-methyl-4-methylenespiro[cyclopentane-1,4'-isochroman]-3'-one (2m)

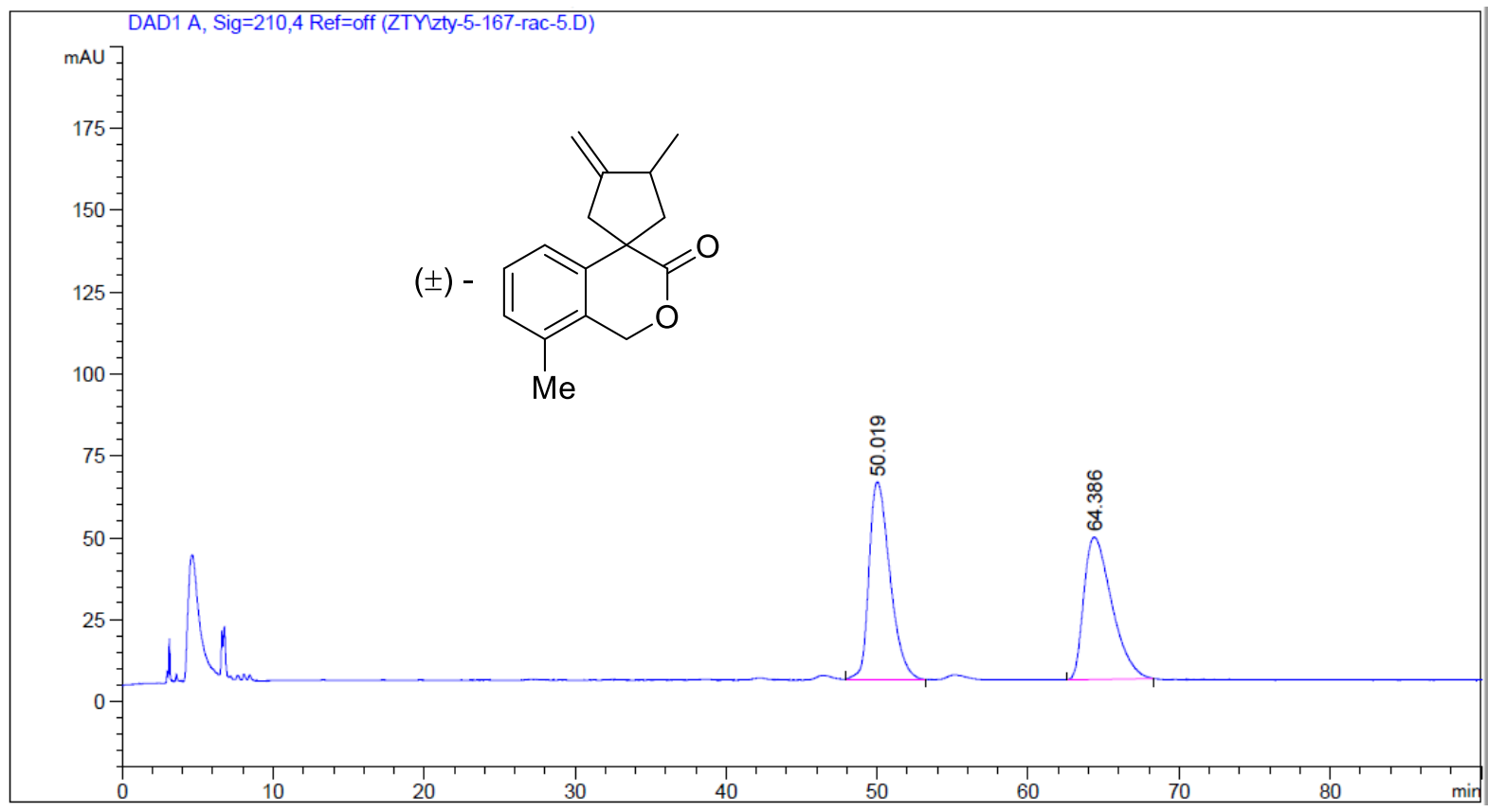

\begin{tabular}{|c|c|c|c|c|c|c|}
\hline $\begin{array}{c}\text { Peak } \\
\#\end{array}$ & $\begin{array}{c}\text { RetTime } \\
\text { [min] }\end{array}$ & Type & $\begin{array}{l}\text { Width } \\
\text { [min] }\end{array}$ & $\begin{array}{c}\text { Area } \\
{\left[\mathrm{mAU}^{*} \mathrm{~s}\right]}\end{array}$ & $\begin{array}{l}\text { Height } \\
{[\mathrm{mAU}]}\end{array}$ & $\begin{array}{c}\text { Area } \\
\%\end{array}$ \\
\hline & & & & - & & - \\
\hline 1 & 50.019 & BB & 1.1756 & 5787.75879 & 60.20298 & 50.8316 \\
\hline 2 & 64.386 & BB & 1.5117 & 5598.38037 & 43.43629 & 49.1684 \\
\hline
\end{tabular}

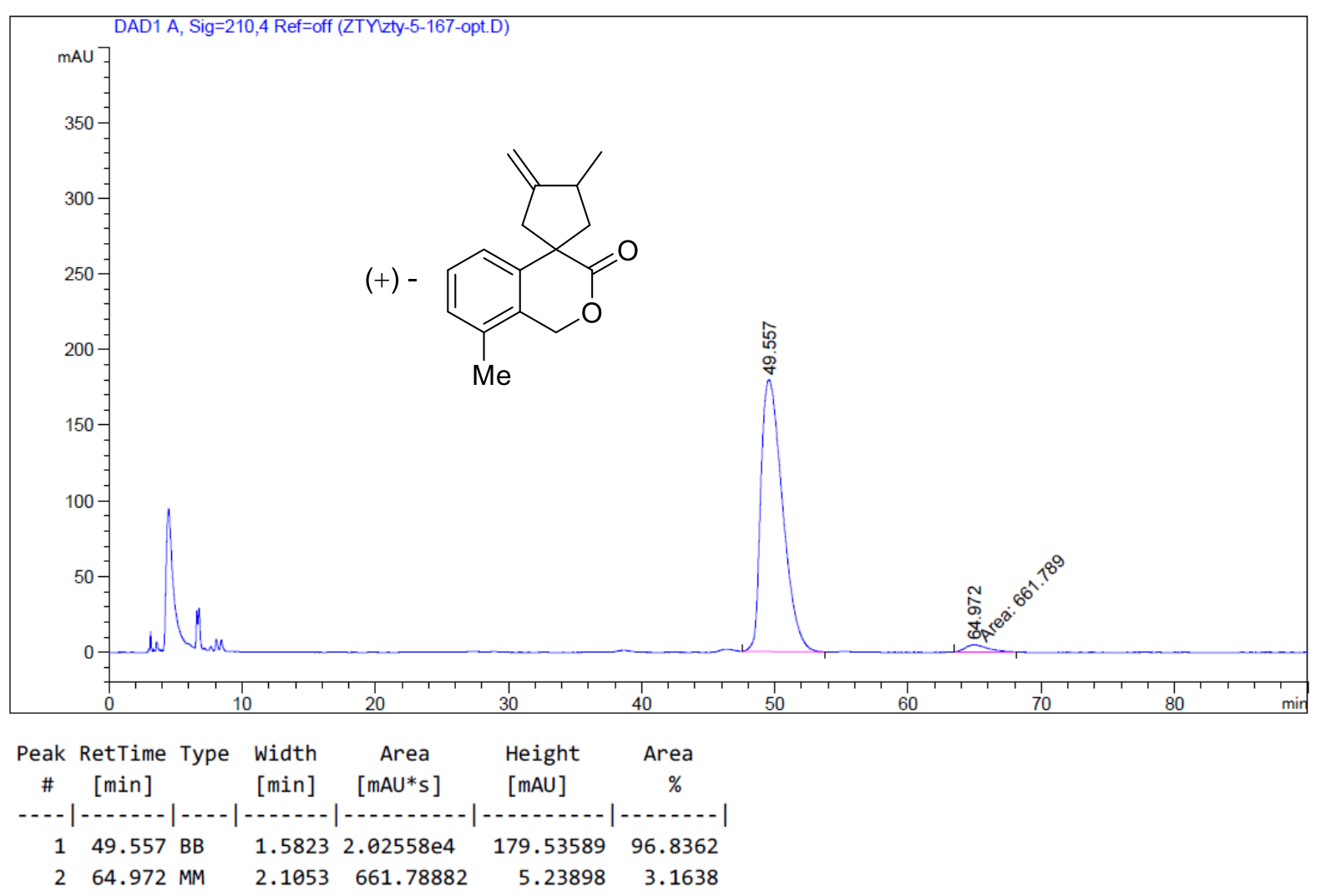


8'-fluoro-3-methyl-4-methylenespiro[cyclopentane-1,4'-isochroman]-3'-one (2n)
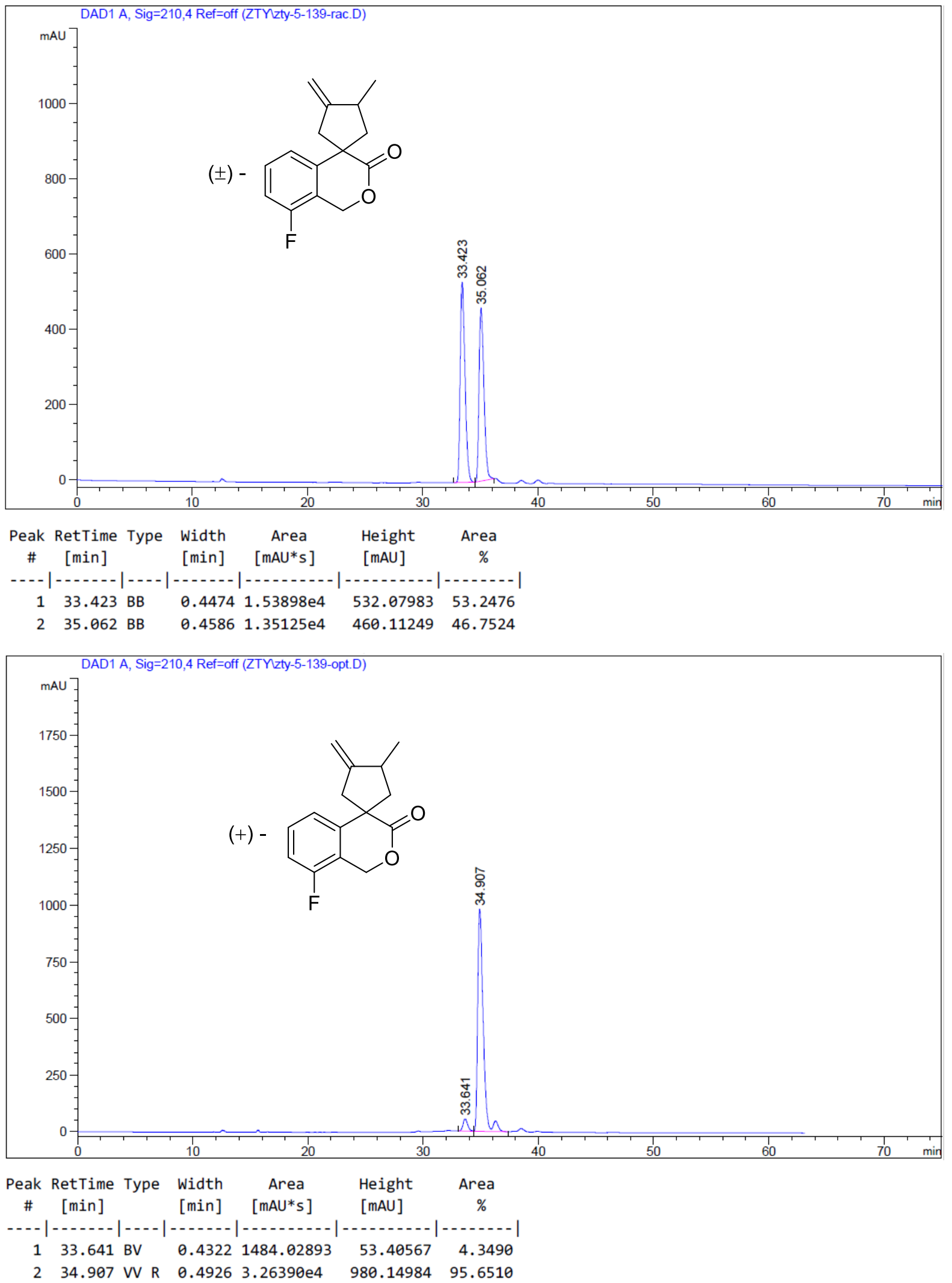
3-methyl-4-methylene-5'H,7'H-spiro[cyclopentane-1,8'-[1,3]dioxolo[4,5-g]isochromen]-

7'-one (20)
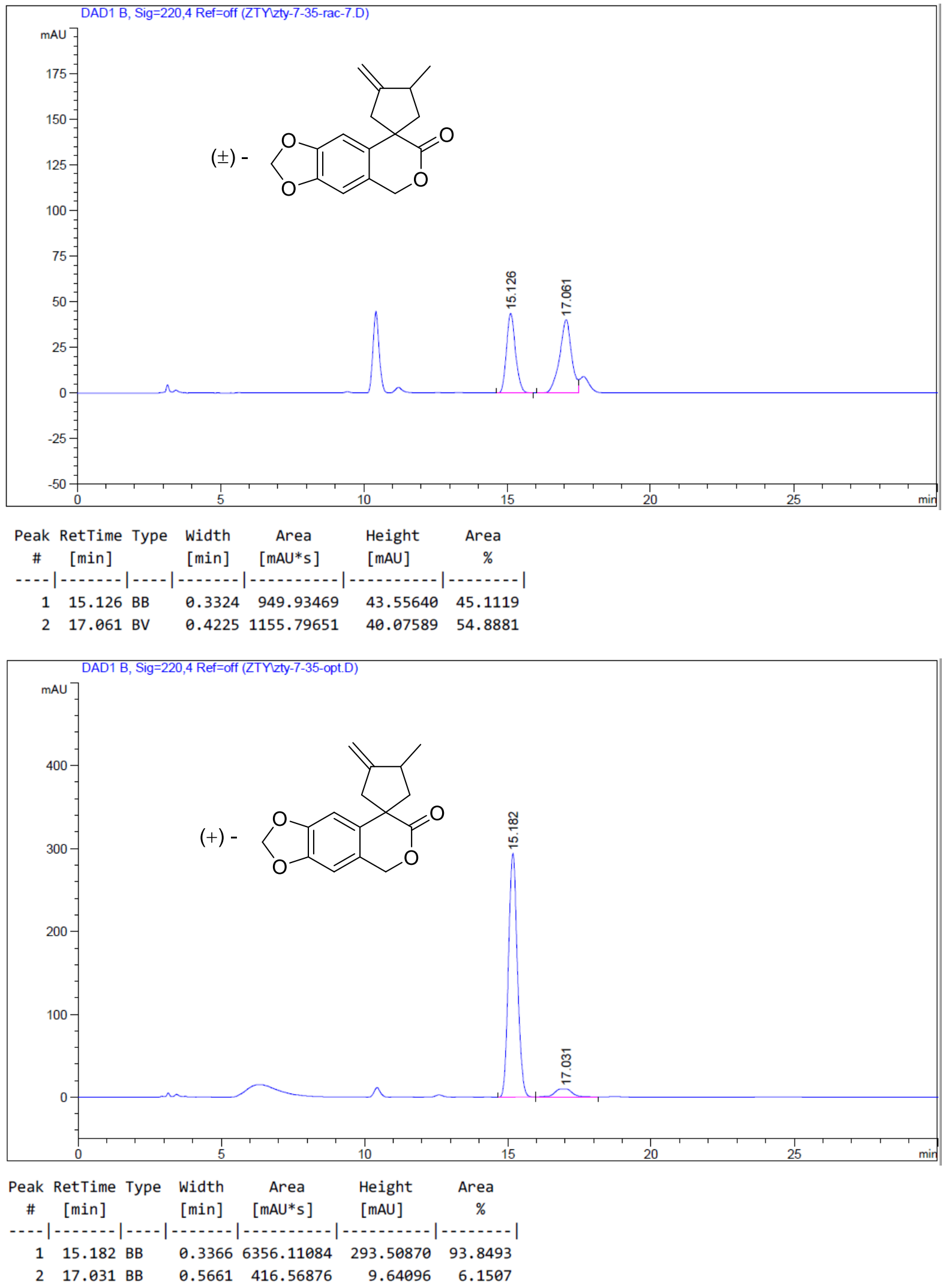


\section{2-allyl-2-(2-(hydroxymethyl)phenyl)pent-4-en-1-ol (2p)}
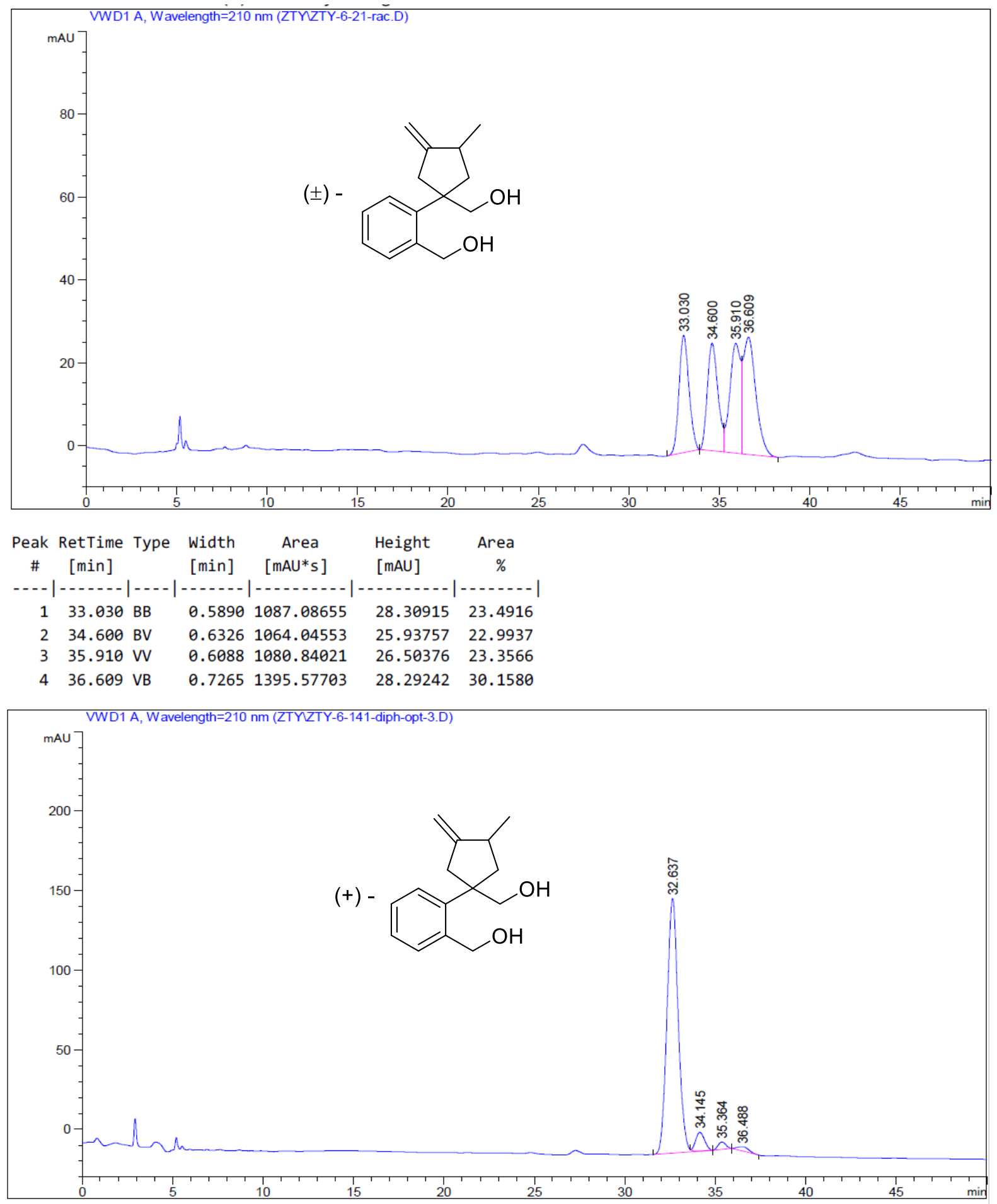

\begin{tabular}{|c|c|c|c|c|c|c|}
\hline $\begin{array}{c}\text { Peak } \\
\#\end{array}$ & $\begin{array}{c}\text { RetTime } \\
\text { [min] }\end{array}$ & Type & $\begin{array}{l}\text { Width } \\
\text { [min] }\end{array}$ & $\begin{array}{c}\text { Area } \\
{\left[\mathrm{mAU}^{*} \mathrm{~s}\right]}\end{array}$ & $\begin{array}{l}\text { Height } \\
{[\mathrm{mAU}]}\end{array}$ & $\begin{array}{c}\text { Area } \\
\%\end{array}$ \\
\hline & & & & & & -- \\
\hline 1 & 32.637 & BV $R$ & 0.6600 & 6872.36133 & 160.03180 & 91.2244 \\
\hline & $\begin{array}{l}34.145 \\
35.364\end{array}$ & $\begin{array}{l}\text { VB E } \\
\text { BB }\end{array}$ & & $\begin{array}{l}405.57181 \\
136.95741\end{array}$ & & $\begin{array}{l}5.3836 \\
1.8180\end{array}$ \\
\hline 4 & 36.488 & BB & 0.6529 & 118.57754 & 2.68664 & 1.5740 \\
\hline
\end{tabular}




\section{(2-(1-(hydroxymethyl)-3-methyl-4-methylenecyclopentyl)-5-methoxyphenyl)methanol}

(2q)
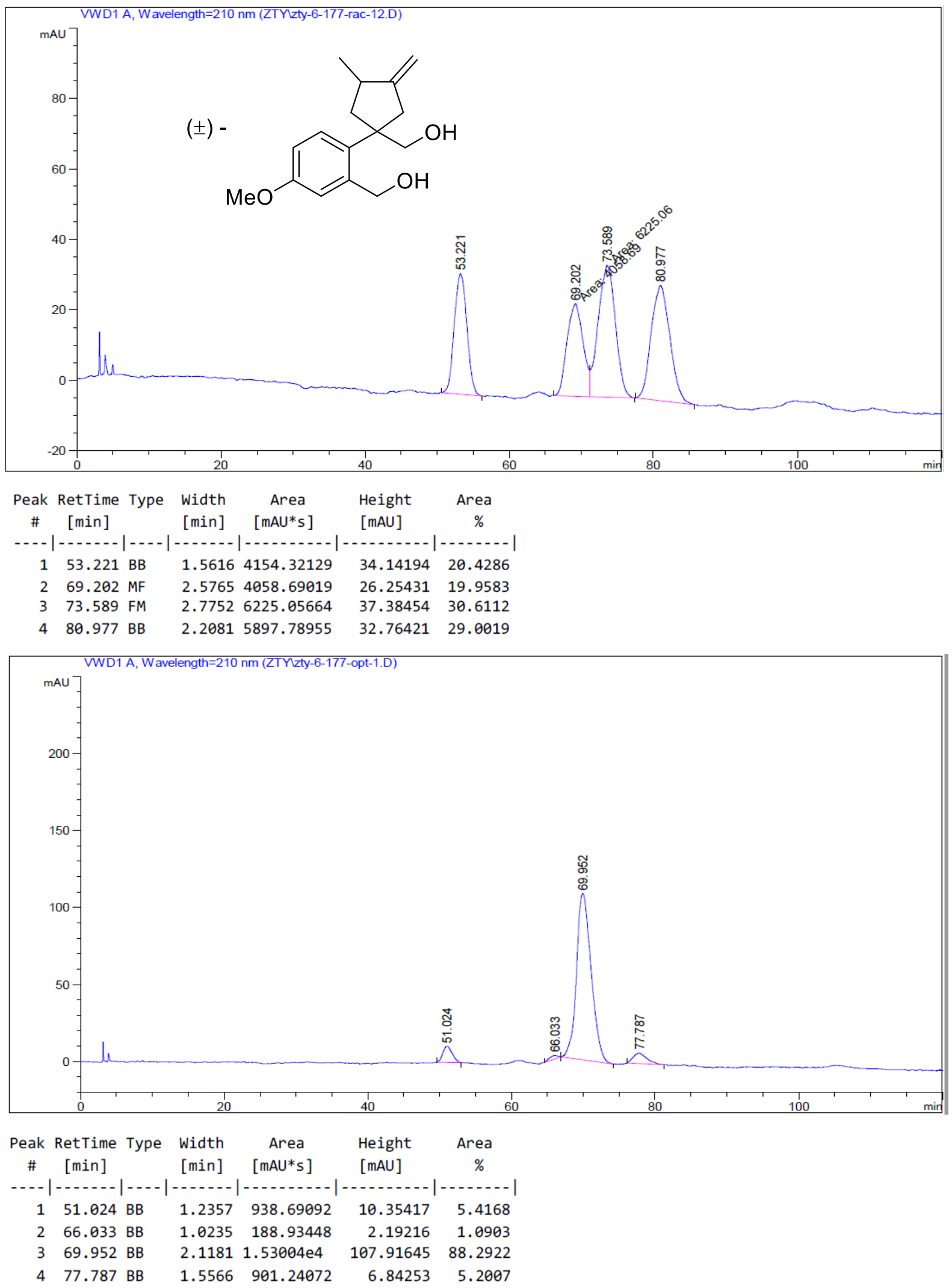

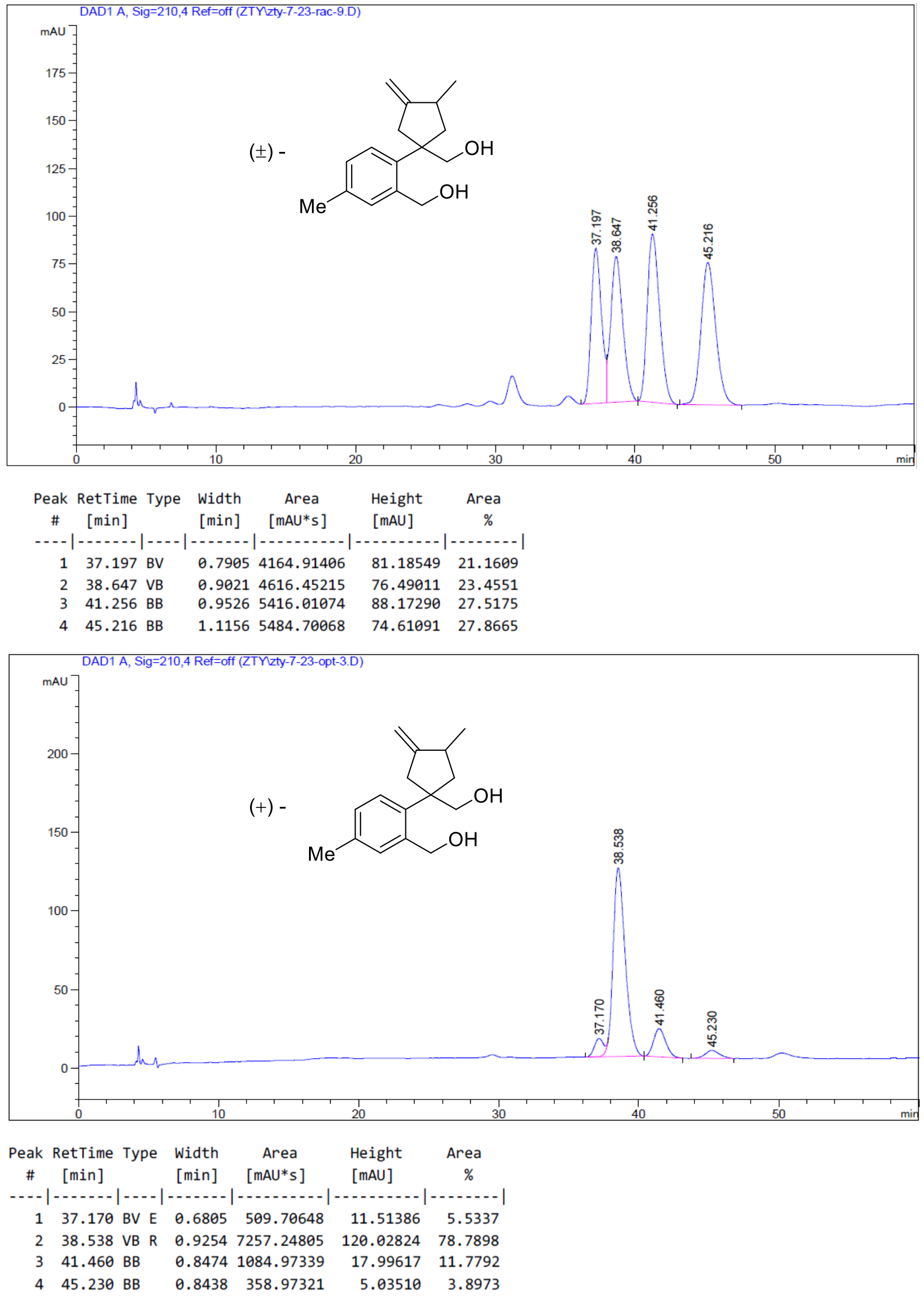
(1-(3-fluoro-2-(hydroxymethyl)phenyl)-3-methyl-4-methylenecyclopentyl)methanol (2s)
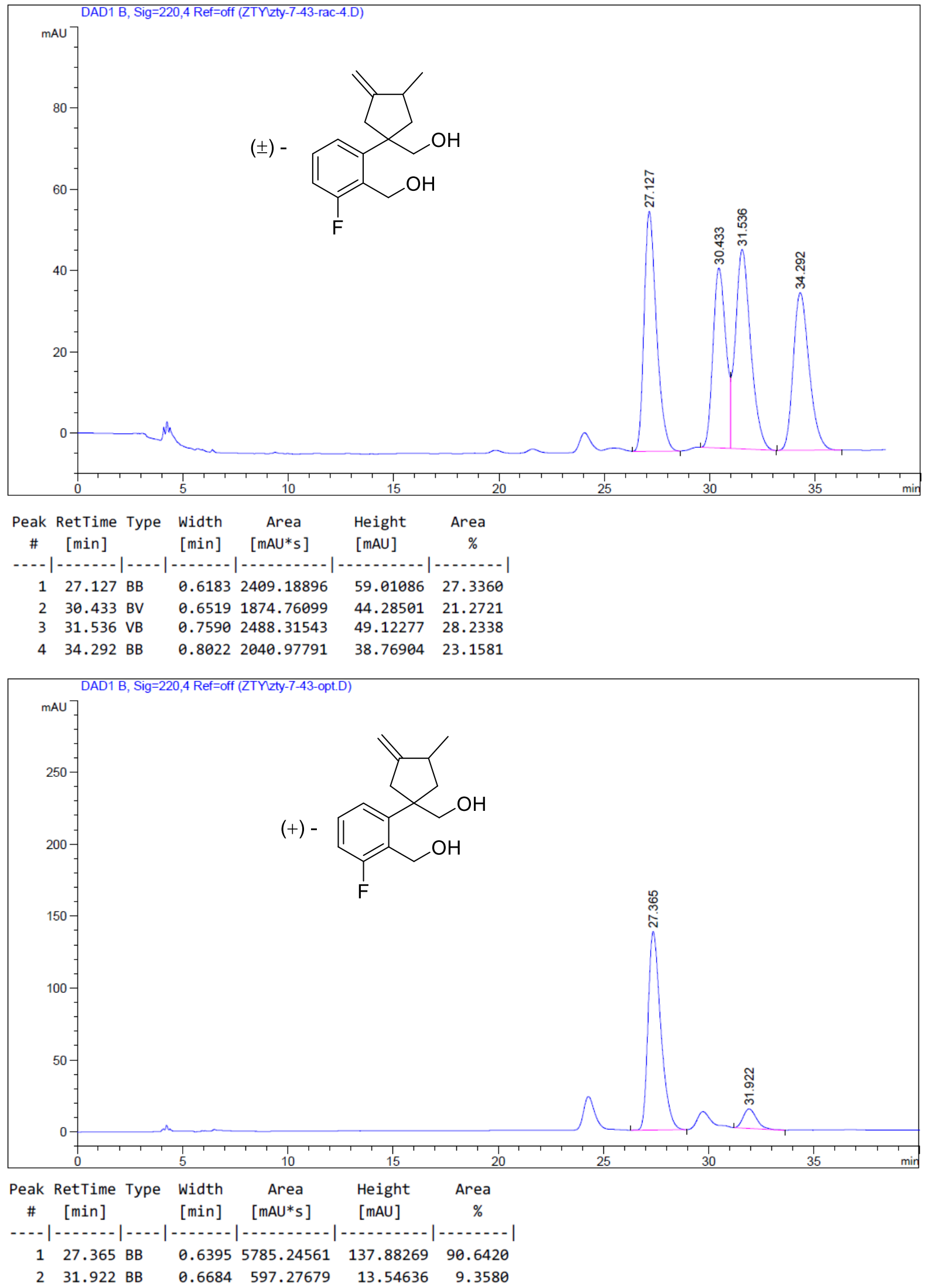
methyl 3-methyl-4-methylene-1-phenylcyclopentane-1-carboxylate (2t)
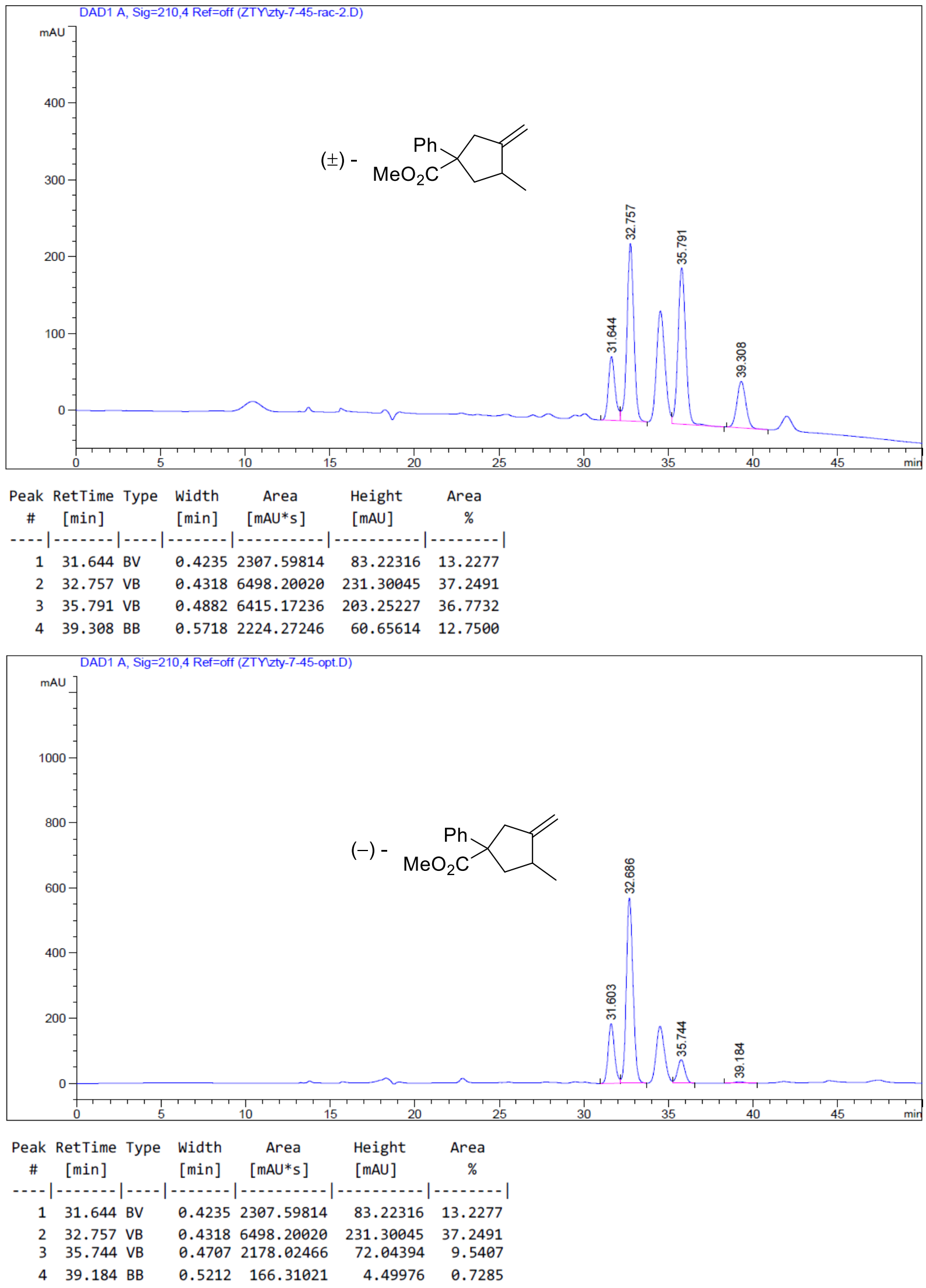
3'-methyl-4'-methylene-2H-spiro[benzofuran-3,1'-cyclopentan]-2-one (2u)
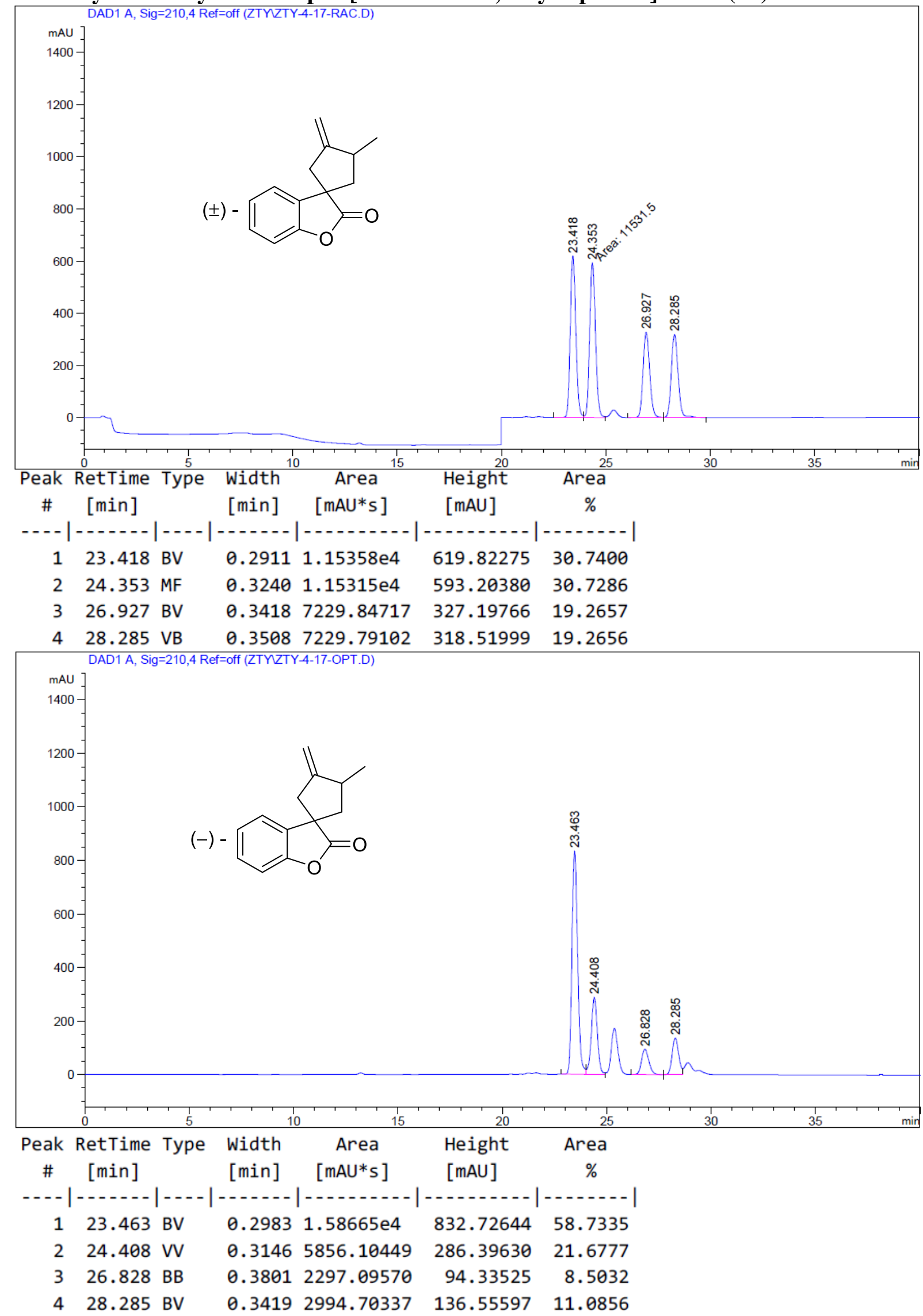


\section{References}

1. Wilke, G.; Bogdanović, B.; Hardt, P.; Heimbach, P.; Keim, W.; Kröner, M.; Oberkirch, W.; Tanaka, K.; Steinrücke, E.; Walter, D.; Zimmermann, H., Allyl-Transition Metal Systems. Angew. Chem. Int. Ed. Engl. 1966, 5 (2), 151-1.

2. Xu, B.; Zhu, S.-F.; Xie, X.-L.; Shen, J.-J.; Zhou, Q.-L., Asymmetric N-H Insertion Reaction Cooperatively Catalyzed by Rhodium and Chiral Spiro Phosphoric Acids. Angew. Chem. Int. Ed. 2011, 50 (48), 11483-11486.

3. Zhu, J.; Li, R.; Su, Y.; Gu, P., Synthesis of Isoindoles from Intramolecular Condensation of Benzyl Azides with $\alpha$-Aryldiazoesters. J. Org. Chem. 2019, 84 (9), 5813-5820.

4. Velcicky, J.; Bodendorf, U.; Rigollier, P.; Epple, R.; Beisner, D. R.; Guerini, D.; Smith, P.; Liu, B.; Feifel, R.; Wipfli, P.; Aichholz, R.; Couttet, P.; Dix, I.; Widmer, T.; Wen, B.; Brandl, T., Discovery of the First Potent, Selective, and Orally Bioavailable Signal Peptide Peptidase-Like 2a (SPPL2a) Inhibitor Displaying Pronounced Immunomodulatory Effects in Vivo. J. Med. Chem. 2018, 61 (3), 865-880.

5. Bray, Katharine L.; Lloyd-Jones, Guy C., Synthesis of [2H,13C]-Labelled Diallylmalonates -Useful Probes for the Study of Transition Metal-Catalysed 1,6-Diene Cycloisomerisation. Eur. J. Org. Chem. 2001, 2001 (9), 1635-1642. 